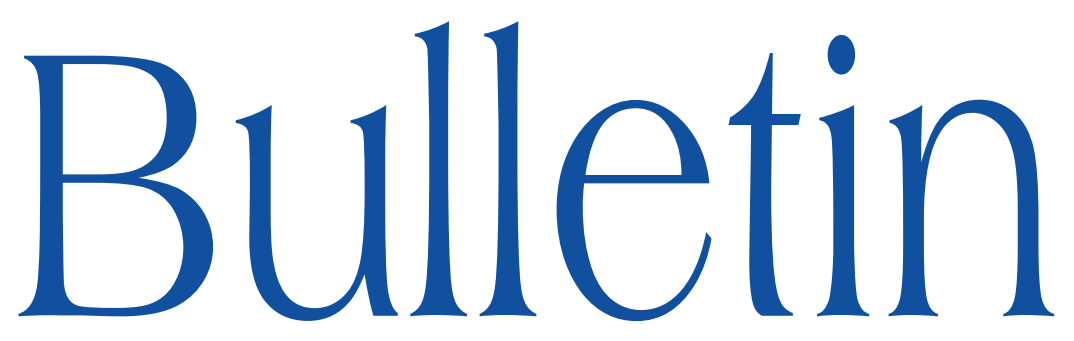

de la SOCIÉTÉ MATHÉMATIQUE DE FRANCE

\title{
D-MODULES ARITHMÉTIQUES ASSOCIÉS AUX ISOCRISTAUX SURCONVERGENTS. CAS LISSE
}

\author{
Daniel Caro
}

\section{Tome 137}

Fascicule 4 


\title{
D-MODULES ARITHMÉTIQUES ASSOCIÉS AUX ISOCRISTAUX SURCONVERGENTS. CAS LISSE
}

\author{
PAR DANIEL CARO
}

\begin{abstract}
RÉSumÉ. - Soient $\mathcal{V}$ un anneau de valuation discrète complet d'inégales caractéristiques, $\mathcal{P}$ un $\mathcal{V}$-schéma formel séparé et lisse, $P$ sa fibre spéciale, $X$ un sous-schéma fermé de $P, T$ un diviseur de $P$ tel que $T_{X}=T \cap X$ soit un diviseur de $X$ et $\mathcal{D}_{\mathcal{P}}^{\dagger}\left({ }^{\dagger} T\right)_{\mathbb{Q}}$ le complété faible du faisceau des opérateurs différentiels sur $\mathcal{P}$ à singularités surconvergentes le long de $T$ tensorisé par $\mathbb{Q}$. Nous construisons un foncteur pleinement fidèle, noté $\operatorname{sp}_{X \hookrightarrow \mathcal{P}, T,+}$, de la catégorie des isocristaux sur $X \backslash T_{X}$ surconvergents le long de

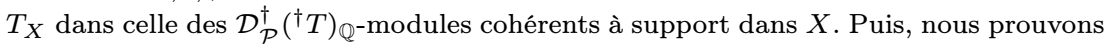
la commutation de $\operatorname{sp}_{X \hookrightarrow \mathcal{P}, T,+}$ aux images inverses (extraordinaires) et aux foncteurs duaux. Ces propriétés sont compatibles à Frobenius.
\end{abstract}

\begin{abstract}
Arithmetic D-modules associated with overconvergent isocrystals. Smooth case)

Let $\mathcal{V}$ be a mixed characteristic complete discrete valuation ring, $\mathcal{P}$ a separated smooth formal scheme over $\mathcal{V}, P$ its special fiber, $X$ a smooth closed subscheme of $P, T$ a divisor in $P$ such that $T_{X}=T \cap X$ is a divisor in $X$ and $\mathcal{D}_{\mathcal{P}}^{\dagger}\left({ }^{\dagger} T\right)_{\mathbb{Q}}$ the tensorized with $\mathbb{Q}$ weak completion of the sheaf of differential operators on $\mathcal{P}$ with overconvergent singularities along $T$. We construct a fully faithful functor denoted by $\operatorname{sp}_{X \hookrightarrow \mathcal{P}, T,+}$ from the category of isocrystal on $X \backslash T_{X}$ overconvergent along $T_{X}$ into the category of coherent $\mathcal{D}_{\mathcal{P}}^{\dagger}\left({ }^{\dagger} T\right)_{\mathbb{Q}}$-modules with support in $X$. Next, we prove the commutation of $\operatorname{sp}_{X \hookrightarrow \mathcal{P}, T,+}$ with (extraordinary) inverse images and dual functors. These properties are compatible with Frobenius.
\end{abstract}

Texte reçu le 18 septembre 2006, accepté le 29 mai 2009

Daniel Caro, Mathématique, Bât. 425, Université Paris-Sud, 91405 Orsay Cedex, France

- E-mail : daniel.caro@math.u-psud.fr

Classification mathématique par sujets (2000). — 14F10, 14F30.

Mots clefs. $-\mathcal{D}$-modules arithmétiques, Frobenius, foncteur dual, image directe.

L'auteur a bénéficié du soutien du réseau européen TMR Arithmetic Algebraic Geometry (contrat numéro UE MRTN-CT-2003-504917). 


\section{Introduction}

Soient $\mathcal{V}$ un anneau de valuation discrète complet d'inégales caractéristiques $(0, p)$ et de corps résiduel $k, \mathcal{P}$ un $\mathcal{V}$-schéma formel séparé et lisse, $P$ sa fibre spéciale, $T$ un diviseur de $P, U:=P \backslash T$ et $F$ la puissance $s$-ième du Frobenius absolu de $P$, avec $s$ un entier fixé. On désigne par $\mathcal{D}_{\mathcal{P}}^{\dagger}\left({ }^{\dagger} T\right)_{\mathbb{Q}}$, l'anneau des opérateurs différentiels sur $\mathcal{P}$ de niveau fini à singularités surconvergentes le long de $T$ (voir [4, 4.2.5]). Lorsque le diviseur $T$ est vide, on ne l'indique pas. On obtient la notion de $\mathcal{D}$-modules arithmétiques sur $U$ à singularités surconvergentes le long de $T$ (lorsque $\mathcal{P}$ est propre, il n'est pas nécessaire de préciser $T)$, i.e., de $\mathcal{D}_{\mathcal{P}}^{\dagger}\left({ }^{\dagger} T\right)_{\mathbb{Q}}$-modules (toujours à gauche par défaut). Pour illustrer ce fait, rappelons que Berthelot a construit un foncteur canonique, noté $\mathrm{sp}_{*}$, pleinement fidèle de la catégorie des isocristaux sur $U$ surconvergent le long de

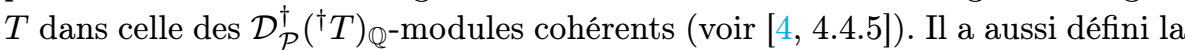
catégorie des $F-\mathcal{D}_{\mathcal{P}}^{\dagger}\left({ }^{\dagger} T\right)_{\mathbb{Q}}$-modules (cohérents) de la façon suivante : les objets sont les couples $(\mathcal{M}, \phi)$, où $\mathcal{M}$ est un $\mathcal{D}_{\mathcal{P}}^{\dagger}\left({ }^{\dagger} T\right)_{\mathbb{Q}^{-}}$module (cohérent) et $\phi$ est un isomorphisme $\mathcal{D}_{\mathcal{P}}^{\dagger}\left({ }^{\dagger} T\right)_{\mathbb{Q}^{-}}$-linéaire $F^{*} \mathcal{M} \stackrel{\sim}{\sim} \mathcal{M}$. Les flèches $(\mathcal{M}, \phi) \rightarrow\left(\mathcal{M}^{\prime}, \phi^{\prime}\right)$ sont les morphismes $\mathcal{D}_{\mathcal{P}}^{\dagger}\left({ }^{\dagger} T\right)_{\mathbb{Q}}$-linéaires $\mathcal{M} \rightarrow \mathcal{M}^{\prime}$ commutant à Frobenius. En construisant de même les $F$-isocristaux surconvergents, il a établi dans [5, 4.6] que le foncteur $\mathrm{sp}_{*}$ commute aux structures de Frobenius respectives.

Soit $X$ un sous-schéma fermé $k$-lisse de $P$ tel que $T \cap X$ soit un diviseur de $X$ (cette dernière hypothèse est inutile mais commode). Dans cet article, nous étendons à cette situation géométrique que l'on appellera « cas de la compactification lisse non relevable » ou simplement « cas lisse » le foncteur $\mathrm{sp}_{*}$ évoqué ci-dessus. Plus précisément, nous construisons un foncteur pleinement fidèle, noté $\operatorname{sp}_{X \hookrightarrow \mathcal{P}, T,+}$, de la catégorie des isocristaux sur $X \backslash(T \cap X)$ surconvergents le long de $T \cap X$ dans celle des $\mathcal{D}_{\mathcal{P}}^{\dagger}\left({ }^{\dagger} T\right)_{\mathbb{Q}^{-}}$modules cohérents à support dans $X$. Lorsque $X \hookrightarrow P$ se relève en un morphisme de $\mathcal{V}$-schémas formels lisses $u$ :

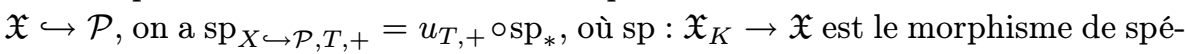
cialisation de la fibre générique de $\mathcal{P}$ (en tant qu'espace analytique rigide) dans $\mathcal{P}$ et où $u_{T,+}$ est l'image directe par $u$ à singularités surconvergentes le long de $T$. Or, comme $X$ est lisse, $X \hookrightarrow P$ se relève localement. La cristallinité de la catégorie des isocristaux surconvergents (resp. des $\mathcal{D}$-modules arithmétiques) sur $X \backslash T$ nous fournit des isomorphismes de recollement entre deux choix de relèvement. La construction de $\operatorname{sp}_{X \hookrightarrow \mathcal{P}, T,+}$ se fait alors par recollement en s'assurant de la compatibilité (et ce de façon transitive) de ces isomorphismes de recollement. Ces résultats avaient été annoncés dans [8]. L'idée de procéder par recollement était bonne mais sa procédure, suite à une erreur décelée par Berthelot, a été amendée dans ce papier.

Avant d'en préciser le contenu, donnons maintenant quelques applications de ce travail initial dans nos autres travaux. Dans ce paragraphe (et seulement 
dans ce paragraphe), $Y$ est toujours lisse mais on ne suppose plus $X$ lisse. Dans [11], nous définissons, au moyen du foncteur $\mathrm{sp}_{+}$de ce papier, une catégorie de $\mathcal{D}$-modules arithmétiques dont les objets sont « les $F$-isocristaux surcohérents sur $Y \gg($ voir $[11,6.2 .1,6.4 .3$.(a)] et les notations de [11, 5.3.1]). Pour étudier (notamment sa stabilité par opérations cohomologiques) cette catégorie de coefficients sur $Y$, l'idée est d'utiliser le théorème de désingularisation de de Jong des variétés algébriques ([22]) pour se ramener au cas où $X$ est lisse (voir les preuves de [11, 6.3.1], [11, 6.3.4]); le cas où $X$ est lisse étant étudié dans ce travail et, pour ce qui concerne la surcohérence de l'image essentielle de $\mathrm{sp}_{+}$, dans $[11,6.1 .4]$. On en déduit, d'abord dans le cas de la désingularisation idéale (voir la définition [11, 7.1.1]), que cette catégorie de coefficients sur $Y$ est équivalente à celle des $F$-isocristaux surconvergents sur $Y$ (voir [11, 7.2.6]). Notons que la preuve de cette équivalence utilise [11, 7.2.4], qui elle-même utilise (voir la première page de la preuve) la description de l'image essentielle du foncteur $\mathrm{sp}_{+}$donnée via 2.5.10 dans ce papier. Puis, on vérifie que cette équivalence entre $F$-isocristaux surcohérents sur $Y$ et $F$-isocristaux surconvergents sur $Y$ se généralise au cas d'une variété quelconque lisse $Y$ (voir [13, 2.3.1]). Grâce aux théorèmes de désingularisation de de Jong et de monodromie génériquement finie et étale de Tsuzuki ([30]), on obtient d'abord la cohérence (4.3.7) puis la surholonomie des $F$-isocristaux surconvergents unités (i.e. dont les pentes de Frobenius sont nulles) sur $Y$ ([15]). En dernier lieu, via le théorème de réduction semi-stable de Kedlaya, (voir [24, 25, 26, 27]), nous étendons ce résultat à tous les $F$-isocristaux surconvergents sur $Y$ (voir d'abord [14] puis [17]).

Précisons à présent le contenu de cet article : dans une première partie, nous établissons la transitivité, pour la composition de morphismes propres, des morphismes d'adjonction entre images directes et images inverses extraordinaires. Nous en déduisons en particulier que l'isomorphisme canonique (i.e., celui construit directement à la main : 1.1.2.1) de composition des images directes utilisé ici est le même (dans le cas de morphismes propres) que celui de $[12,1.2 .15]$ dont la construction nécessite le théorème de dualité relative. D'où une unification (voir 1.2.9).

Dans le deuxième chapitre, nous construisons le foncteur pleinement fidèle $\operatorname{sp}_{X \hookrightarrow \mathcal{P}, T,+} \cdot$

Dans [10], nous avons obtenu un isomorphisme de commutation de $\mathrm{sp}_{*}$ aux foncteurs duaux respectifs. Pour l'étendre à $\operatorname{sp}_{X \hookrightarrow \mathcal{P}, T,+}$, il s'agit d'abord d'établir sa compatibilité aux foncteurs images inverses (extraordinaires) par une immersion ouverte. Cela fait l'objet de la troisième partie.

Dans le quatrième chapitre, nous prouvons la commutation de $\operatorname{sp}_{X \hookrightarrow \mathcal{P}, T,+}$ aux foncteurs restrictions, images inverses extraordinaires et foncteurs duaux. La vérification la plus technique est celle concernant le foncteur dual. Nous construisons pour cela un isomorphisme de commutation des foncteurs duaux 
aux images inverses extraordinaires par une immersion. Pour valider sa transitivité, nous utilisons celle prouvée dans le premier chapitre. Dans sa procédure de recollement, nous nous servons aussi du troisième chapitre.

Le théorème de pleine fidélité de Kedlaya du foncteur associant à des $F$ isocristaux sur $Y$ surconvergents le long de $T \cap X$ les $F$-isocristaux convergents sur $Y$ correspondants (voir [25, 4.2.1]) et celui de Tsuzuki sur les restrictions (voir [30, 4.1.1]) simplifient, lorsque l'on se restreint aux $F$-isocristaux surconvergents, la vérification de la commutation de $\operatorname{sp}_{X \hookrightarrow \mathcal{P}, T,+}$ aux foncteurs cohomologiques ci-dessus. Ces théorèmes nous ramènent sans difficulté au cas où $X \hookrightarrow P$ se relève, ce qui évite les tracas engendrés par les recollements (transitivité etc.). Ainsi, lorsque l'on dispose de structures de Frobenius, le premier et le troisième chapitre deviennent superflus.

Enfin, nous terminons par la cohérence, en tant que $\mathcal{D}_{\mathcal{P}, \mathbb{Q}}^{\dagger}$-module, de l'image par $\operatorname{sp}_{X \hookrightarrow \mathcal{P}, T,+}$ d'un $F$-isocristal unité sur $X \backslash(T \cap X)$ surconvergent le long de $T \cap X$.

Remerciements. - Je remercie P. Berthelot pour une erreur qu'il a décelée dans la précédente procédure de recollement permettant de construire $\operatorname{sp}_{X \hookrightarrow \mathcal{P}, T,+}$.

\section{Notations}

Nous garderons les notations suivantes : les schémas formels seront notés par des lettres calligraphiques ou gothiques et leur fibre spéciale par les lettres romanes correspondantes. De plus, la lettre $\mathcal{V}$ désignera un anneau de valuation discrète complet, de corps résiduel $k$ de caractéristique $p>0$, de corps de fractions $K$ de caractéristique 0 , d'idéal maximal $\mathfrak{m}$ et $\pi$ une uniformisante. On fixe un entier naturel $s \geq 1$ et on désigne par $F$ la puissance $s$-ième de l'endomorphisme de Frobenius. Les modules sont par défaut des modules à gauche. Si $X$ est un schéma ou schéma formel, on note $d_{X}$ la dimension de Krull de $X$ et $\omega_{X}=\Omega_{X}^{d_{X}}$ le faisceau des différentielles de degré maximum. S'il existe un système de coordonnées locales $t_{1}, \ldots, t_{d}$ (sur un schéma ou schéma formel), on notera, pour tous $i=1, \ldots, d, \tau_{i}=1 \otimes t_{i}-t_{i} \otimes 1$ et $\partial_{i}$ les dérivations correspondantes. Pour tout $\underline{k} \in \mathbb{N}^{d}$, on pose $\underline{\tau}^{\{\underline{k}\}}:=\tau_{1}^{\left\{k_{1}\right\}} \cdots \tau_{d}^{\left\{k_{d}\right\}}, \underline{\partial}^{<\underline{k}>}:=$ $\partial_{1}^{<k_{1}>} \ldots \partial_{d}^{<k_{d}>}$. Si $\mathcal{E}$ est un faisceau abélien, on écrit $\mathcal{E}_{\mathbb{Q}}:=\mathcal{E} \otimes_{\mathbb{Z}} \mathbb{Q}$.

- Les indices qc, tdf, coh et parf signifient respectivement quasi-cohérent, de Tor-dimension finie, cohérent et parfait tandis que $D^{\mathrm{b}}, D^{+}$et $D^{-}$désignent respectivement les catégories dérivées des complexes à cohomologie bornée, bornée inférieurement et bornée supérieurement. Enfin, si $\mathcal{A}$ est un faisceau d'anneaux, les symboles ${ }^{g} \mathcal{A},{ }^{d} \mathcal{A}$ puis ${ }^{*} \mathcal{A}$ se traduisent respectivement par $\mathcal{A}$ module « à gauche », « à droite » puis « à droite ou à gauche » (par exemple, $D\left({ }^{g} \mathcal{A}\right)$ indique la catégorie dérivée des complexes de $\mathcal{A}$-modules à gauche). 
Si $\mathcal{A}$ et $\mathcal{B}$ sont deux faisceaux d'anneaux (sur un même espace topologique), on notera $D_{(., \mathrm{qc})}\left({ }^{*} \mathcal{A},{ }^{*} \mathcal{B}\right)$, la sous catégorie pleine de $D\left({ }^{*} \mathcal{A},{ }^{*} \mathcal{B}\right)$ formée des complexes quasi-cohérents à droite. De même en mettant le point à droite et en remplaçant «à droite » par « à gauche »; de même en remplaçant respectivement «qc» par «tdf » et «quasi-cohérent» par « de Tor-dimension finie» etc. Comme les catégories de complexes sont par défaut les catégories dérivées, on écrit $\operatorname{Hom}_{\mathcal{A}}(-,-)$ pour $\operatorname{Hom}_{D(\mathcal{A})}(-,-)$.

Soit $f: \mathcal{P}^{\prime} \rightarrow \mathcal{P}$ un morphisme de $\mathcal{V}$-schémas formels lisses, $T$ un diviseur de $P$ tel que $f^{-1}(T)$ soit un diviseur de $P^{\prime}$. On notera $f_{i}: P_{i}^{\prime} \rightarrow P_{i}$, la réduction de $f$ modulo $\mathfrak{m}^{i+1}$. En particulier, on obtient $f_{0}: P^{\prime} \rightarrow P$. Pour tout entier $m \geq 0$, nous reprenons les constructions de Noot-Huyghe (voir [21, 2.1]) concernant les faisceaux de la forme $\mathcal{B}_{P_{i}}^{(m)}(T)$ ou $\mathcal{B}_{\mathcal{P}}^{(m)}(T)$. En notant $\mathcal{D}_{\mathcal{P}}^{(m)}(T):=\mathcal{B}_{\mathcal{P}}^{\left(n_{m}\right)}(T) \otimes_{\mathcal{O}_{\mathcal{P}}} \mathcal{D}_{\mathcal{P}}^{(m)}$, avec $\left(n_{m}\right)_{m \in \mathbb{N}}$ une suite d'entiers telle que $n_{m} \geq m$, on construit la catégorie des complexes quasi-cohérents $\underset{L}{L} D_{\mathbb{Q}, \mathrm{qc}}^{\mathrm{b}}\left(\widehat{\mathcal{D}}_{\mathcal{P}}^{(\bullet)}(T)\right)$ (voir $[12,1.1]$ et $\left.[6,4.2]\right)$. On prendra par défaut la suite $n_{m}=m$. On écrira $\mathcal{O}_{\mathcal{P}}\left({ }^{\dagger} T\right)_{\mathbb{Q}}$ pour le faisceau des fonctions sur $\mathcal{P}$ à singularités surconvergentes le long de $T$ (voir [4, 4.2.4.2]). Les produits tensoriels internes pour ces complexes quasi-cohérents seront notés $-\otimes_{\mathcal{O}_{\mathcal{P}}\left({ }^{\dagger} T\right)_{\mathbb{Q}}}^{\dagger}$ (voir 4.3.2). On note $\left.\lim _{\longrightarrow}: \underset{D_{\mathbb{Q}}, \mathrm{qc}}{\mathrm{b}}\left(\widehat{\mathcal{D}}_{\mathcal{P}}^{(\bullet)}(T)\right) \rightarrow D\left(\mathcal{D}_{\mathcal{P}, \mathbb{Q}}^{\dagger}{ }^{\dagger} T\right)\right)$ le foncteur canonique (voir [6, 4.2.2] lorsque le diviseur est vide, mais la construction est similaire). De plus, nous désignerons respectivement par $f_{T}^{!}, f_{T+}$ les foncteurs image inverse extraordinaire, image directe par $f$ à singularités surconvergentes le long de $T$ (voir $[6,3.4,3.5,4.3]$ et $[12,1.1 .5]$ ). Pour ces deux opérations cohomologiques, lorsque l'on aura affaire à des complexes de bimodules, pour préciser quelle structure nous choisissons dans leur calcul, on mettra un indice « $g$ » (resp. « $d »)$ en bas pour indiquer la structure gauche (resp. droite). En outre, si $Z$ est un sous-schéma fermé de $P, \mathbb{R} \underline{\Gamma}_{Z}^{\dagger}$ sera le foncteur cohomologique local à support strict dans $Z$ (au sens de $[9,2.2 .6]$ ) et $\left({ }^{\dagger} Z\right.$ ) le foncteur restriction ([9, 2.2.6]). Si $T^{\prime} \subset T$ sont deux diviseurs de $P$, on notera abusivement $\left({ }^{\dagger} T\right.$ ) à la place de $\left({ }^{\dagger} T, T^{\prime}\right)$ (voir $\left.[12,1.1 .6 .4]\right)$. Pour tout diviseur $T$ de $P$, nous désignerons par $\mathbb{D}_{\mathcal{P}, T}^{\dagger}, \mathbb{D}_{\mathcal{P}, T}$ ou simplement par $\mathbb{D}_{T}$, le foncteur dual $\mathcal{D}_{\mathcal{P}}^{\dagger}\left({ }^{\dagger} T\right)_{\mathbb{Q}}$-linéaire (voir 3.2.1). Lorsque $T$ est l'ensemble vide, nous omettrons de l'indiquer dans les opérations cohomologiques ci-dessus.

\section{Convention}

Soit $\mathcal{A}$ un faisceau d'anneaux et $\mathcal{E}$ un $\mathcal{A}$-bimodule à gauche. Pour calculer les termes de la forme $-\otimes_{\mathcal{A}} \mathcal{E}$ ou $\mathcal{H o m}_{\mathcal{A}}(-, \mathcal{E})$, on prendra par défaut la structure gauche de $\mathcal{E}$. De plus, si $\mathcal{M}$ est un $\mathcal{A}$-bimodule à droite, pour calculer $\mathcal{M} \otimes_{\mathcal{A}}-$ 
(resp. $\mathcal{H}_{\mathcal{A}}(-, \mathcal{M})$ ), nous choisissons par défaut la structure droite (resp. gauche) de $\mathcal{M}$. De même pour les complexes.

\section{Transitivité du morphisme d'adjonction entre image directe et image inverse extraordinaire}

Nous vérifions ici que la transitivité pour la composition de morphismes propres de l'isomorphisme de dualité relative implique celle des morphismes d'adjonction entre image directe et image inverse extraordinaire. Cette transitivité nous permettra dans un prochain chapitre d'établir la transitivité de l'isomorphisme de commutation des foncteurs duaux aux images inverses extraordinaires par une immersion (voir 4.2.8).

1.1. Cas des schémas. - Soient $S$ un schéma noethérien de dimension de Krull finie, $f: Y \rightarrow X$ et $g: Z \rightarrow Y$ deux $S$-morphismes propres de $S$-schémas lisses. On rappelle que $\mathcal{D}_{Y \rightarrow X}^{(m)}$ est le $\left(\mathcal{D}_{Y}^{(m)}, f^{-1} \mathcal{D}_{X}^{(m)}\right)$-bimodule $f^{*} \mathcal{D}_{X}^{(m)}$, où $f^{*}$ désigne l'image inverse en tant que $\mathcal{O}_{X}$-module. Pour la définition des images inverses extraordinaires et images directes, nous renvoyons à [6, 2.2 et 2.3]. On pourra identifier $f^{!} \mathcal{D}_{X}^{(m)}$ avec $\mathcal{D}_{Y \rightarrow X}^{(m)}\left[d_{Y / X}\right]$.

1.1.1. - Soient $\mathcal{A}_{X}$ un faisceau d'anneaux sur $X$. Comme $Y$ est noethérien, le foncteur $f_{*}$ est de dimension cohomologique finie et induit le foncteur $\mathbb{R} f_{*}$ : $D\left(f^{-1} \mathcal{A}_{X}{ }^{\mathrm{d}}\right) \rightarrow D\left(\mathcal{A}_{X}{ }^{\mathrm{d}}\right)$. Pour tous $\mathcal{E} \in D_{\text {tdf,qc }}\left({ }^{\mathrm{g}} \mathcal{A}_{X}\right)$ et $\mathcal{F} \in D\left(f^{-1} \mathcal{A}_{X}{ }^{\mathrm{d}}\right)$, on notera

$$
\operatorname{proj}_{f}: \mathbb{R} f_{*} \mathcal{F} \otimes_{\mathcal{A}_{X}}^{\mathbb{L}} \mathcal{E} \stackrel{\sim}{\longrightarrow} \mathbb{R} f_{*}\left(\mathcal{F} \otimes_{f^{-1} \mathcal{A}_{X}}^{\mathbb{L}} f^{-1} \mathcal{E}\right)
$$

l'isomorphisme de projection ou ceux qui s'en déduisent par fonctorialité. De même, en remplaçant $f$ par $g$ etc. Il pourra aussi se noter proj.

De plus, nous désignerons par $\otimes$ les isomorphismes de la forme [10, 2.1.12.(i)] ou ceux qui s'en déduisent par fonctorialité.

1.1.2 (Isomorphismes canoniques de composition). — Les images directes par $f$ de complexes de $\mathcal{D}_{Y}$-modules à droite (resp. à gauche) seront notés $f_{+}^{\text {d }}$ (resp. $\left.f_{+}^{\mathrm{g}}\right)$. Si aucune ambiguïté n'est à craindre, on les notera simplement $f_{+}$.

Pour tout $\mathcal{G} \in D_{\text {coh }}^{\mathrm{b}}\left(\mathcal{D}_{Z}^{(m) \mathrm{d}}\right)$, on dispose d'un isomorphisme canonique $f_{+}^{\mathrm{d}} \circ$ $g_{+}^{\mathrm{d}}(\mathcal{G}) \stackrel{\sim}{\longrightarrow}(f \circ g)_{+}^{\mathrm{d}}(\mathcal{G})$ fonctoriel en $\mathcal{G}$. Celui-ci est le composé suivant :

$$
\begin{gathered}
f_{+}^{\mathrm{d}} \circ g_{+}^{\mathrm{d}}(\mathcal{G})=\mathbb{R} f_{*}\left(\mathbb{R} g_{*}\left(\mathcal{G} \otimes_{\mathcal{D}_{Z}^{(m)}}^{\mathbb{L}} \mathcal{D}_{Z \rightarrow Y}^{(m)}\right) \otimes_{\mathcal{D}_{Y}^{(m)}}^{\mathbb{L}} \mathcal{D}_{Y \rightarrow X}^{(m)}\right) \\
\underset{\operatorname{proj}_{g}}{\sim} \mathbb{R} f_{*} \mathbb{R} g_{*}\left(\mathcal{G} \otimes_{\mathcal{D}_{Z}^{(m)}}^{\mathbb{L}} \mathcal{D}_{Z \rightarrow Y}^{(m)} \otimes_{g^{-1} \mathcal{D}_{Y}^{(m)}}^{\mathbb{L}} g^{-1} \mathcal{D}_{Y \rightarrow X}^{(m)}\right) \stackrel{\sim}{\longrightarrow} \mathbb{R} f_{*} \mathbb{R} g_{*}\left(\mathcal{G} \otimes_{\mathcal{D}_{Z}^{(m)}}^{\mathbb{L}} \mathcal{D}_{Z \rightarrow X}^{(m)}\right) \\
\stackrel{\sim}{\longrightarrow}(f \circ g)_{+}^{\mathrm{d}}(\mathcal{G}),
\end{gathered}
$$


où l'avant dernier isomorphisme découle de l'isomorphisme canonique $\mathcal{D}_{Z \rightarrow Y}^{(m)} \otimes_{g^{-1} \mathcal{D}_{Y}^{(m)}}^{\mathbb{L}} g^{-1} \mathcal{D}_{Y \rightarrow X}^{(m)} \stackrel{\sim}{\longrightarrow} \mathcal{D}_{Z \rightarrow X}^{(m)}$. En tordant les structures de droite à gauche, il en découle, pour tout $\mathcal{G} \in D_{\text {coh }}^{\mathrm{b}}\left({ }^{\mathrm{g}} \mathcal{D}_{Z}^{(m)}\right)$, l'isomorphisme canonique $f_{+}^{\mathrm{g}} \circ g_{+}^{\mathrm{g}}(\mathcal{G}) \stackrel{\sim}{\longrightarrow}(f \circ g)_{+}^{\mathrm{g}}(\mathcal{G})$.

De plus, si $\mathcal{E} \in D_{\text {coh }}^{\mathrm{b}}\left({ }^{\mathrm{g}} \mathcal{D}_{X}^{(m)}\right)$, on dispose de $g^{!} \circ f^{!}(\mathcal{E}) \stackrel{\sim}{\longrightarrow}(f \circ g)^{!}(\mathcal{E})$ via les isomorphismes :

$$
\begin{aligned}
& g^{!} f^{!}(\mathcal{E})=g^{!} \mathcal{D}_{Y}^{(m)} \otimes_{g^{-1} \mathcal{D}_{Y}^{(m)}}^{\mathbb{L}} g^{-1}\left(f^{!} \mathcal{D}_{X}^{(m)} \otimes_{f^{-1} \mathcal{D}_{X}^{(m)}}^{\mathbb{L}} f^{-1} \mathcal{E}\right) \\
& \stackrel{\sim}{\longrightarrow} g^{!} \mathcal{D}_{Y}^{(m)} \otimes_{g^{-1}}^{\mathbb{L}} \mathcal{D}_{Y}^{(m)} g^{-1} f^{!} \mathcal{D}_{X}^{(m)} \otimes_{g^{-1} f^{-1} \mathcal{D}_{X}^{(m)}}^{\mathbb{L}} g^{-1} f^{-1} \mathcal{E}=g^{!} f^{!} \mathcal{D}_{X}^{(m)} \otimes_{g^{-1} f^{-1}}^{\mathbb{L}} \mathcal{D}_{X}^{(m)} g^{-1} f^{-1} \mathcal{E} \\
& \stackrel{\sim}{\longrightarrow}(f \circ g)^{!} \mathcal{D}_{X}^{(m)} \otimes_{g^{-1} f^{-1}}^{\mathbb{L}} \mathcal{D}_{X}^{(m)} g^{-1} f^{-1} \mathcal{E} \stackrel{\sim}{\longrightarrow}(f \circ g)^{!}(\mathcal{E}) .
\end{aligned}
$$

1.1.3. - Soient $\mathcal{E} \in D_{\mathrm{qc}, \mathrm{tdf}}^{\mathrm{b}}\left({ }^{\mathrm{g}} \mathcal{D}_{X}^{(m)}\right)$ et $\mathcal{F} \in D_{\text {coh }}^{\mathrm{b}}\left({ }^{\mathrm{g}} \mathcal{D}_{Y}^{(m)}\right)$. D'après Virrion (voir [32]), on dispose de l'isomorphisme

$$
f_{+}^{\mathrm{d}} \mathbb{R} \mathcal{H o m}_{\mathcal{D}_{Y}^{(m)}}\left(\mathcal{F}, \mathcal{D}_{Y}^{(m)}\right)\left[d_{Y}\right] \stackrel{\sim}{\longrightarrow} \mathbb{R} \mathcal{H} o m_{\mathcal{D}_{X}^{(m)}}\left(f_{+}^{\mathrm{g}}(\mathcal{F}), \mathcal{D}_{X}^{(m)}\right)\left[d_{X}\right]
$$

que l'on notera ici $\chi_{f}^{\prime}$. Elle en déduit le composé suivant :

$$
\mathbb{R H o m}_{\mathcal{D}_{X}^{(m)}}\left(f_{+}(\mathcal{F}), \mathcal{E}\right)
$$

$\underset{\otimes}{\tilde{\otimes}} \mathbb{R} \mathcal{H} o m_{\mathcal{D}_{X}^{(m)}}\left(f_{+}(\mathcal{F}), \mathcal{D}_{X}^{(m)}\right) \otimes_{\mathcal{D}_{X}^{(m)}}^{\mathbb{L}} \mathcal{E} \underset{\chi_{f}^{\prime}}{\sim} f_{+} \mathbb{R} \mathcal{H} o m_{\mathcal{D}_{Y}^{(m)}}\left(\mathcal{F}, \mathcal{D}_{Y}^{(m)}\right) \otimes_{\mathcal{D}_{X}^{(m)}}^{\mathbb{L}} \mathcal{E}\left[d_{Y / X}\right]$ $=\mathbb{R} f_{*}\left(\mathbb{R} \mathcal{H} o m_{\mathcal{D}_{Y}^{(m)}}\left(\mathcal{F}, \mathcal{D}_{Y}^{(m)}\right) \otimes_{\mathcal{D}_{Y}^{(m)}}^{\mathbb{L}} f^{!} \mathcal{D}_{X}^{(m)}\right) \otimes_{\mathcal{D}_{X}^{(m)}}^{\mathbb{L}} \mathcal{E} \underset{\otimes}{\sim} \mathbb{R} f_{*} \mathbb{R} \mathcal{H} o m_{\mathcal{D}_{Y}^{(m)}}\left(\mathcal{F}, f^{!} \mathcal{D}_{X}^{(m)}\right) \otimes_{\mathcal{D}_{X}^{(m)}}^{\mathbb{L}} \mathcal{E}$

$$
\underset{\operatorname{proj}_{f}}{\stackrel{\sim}{\longrightarrow}} \mathbb{R} f_{*}\left(\mathbb{R} \mathcal{H o m} \mathcal{D}_{Y}^{(m)}\left(\mathcal{F}, f^{!} \mathcal{D}_{X}^{(m)}\right) \otimes_{f^{-1} \mathcal{D}_{X}^{(m)}}^{\mathbb{L}} f^{-1} \mathcal{E}\right)
$$

$$
\underset{\otimes}{\stackrel{\longrightarrow}{R} f_{*} \mathbb{R} \mathcal{H o m}}{ }_{\mathcal{D}_{Y}^{(m)}}\left(\mathcal{F}, f^{!} \mathcal{E}\right)
$$

que l'on notera

$$
\delta_{f}: \mathbb{R}_{\mathcal{H} o m_{\mathcal{D}_{X}^{(m)}}}\left(f_{+}(\mathcal{F}), \mathcal{E}\right) \stackrel{\sim}{\longrightarrow} \mathbb{R} f_{*} \mathbb{R} \mathcal{H} o m_{\mathcal{D}_{Y}^{(m)}}\left(\mathcal{F}, f^{!} \mathcal{E}\right) .
$$

Lemme 1.1.4. - Soient $\mathcal{E} \in D_{\mathrm{qc}, \mathrm{tdf}}^{\mathrm{b}}\left({ }^{\mathrm{g}} \mathcal{D}_{X}^{(m)}\right)$ et $\mathcal{F} \in D_{\mathrm{coh}}^{\mathrm{b}}\left({ }^{\mathrm{g}} \mathcal{D}_{Y}^{(m)}\right)$. Le diagramme suivant :

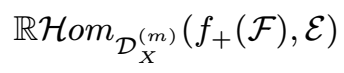

$$
\begin{aligned}
& \downarrow \delta_{f} \\
& \mathbb{R} \mathcal{H} o m_{\mathcal{D}_{X}^{(m)}}\left(f_{+}(\mathcal{F}), \mathcal{D}_{X}^{(m)}\right) \otimes_{\mathcal{D}_{X}^{(m)}}^{\mathbb{L}} \mathcal{E} \\
& \downarrow \delta_{f} \\
& \mathbb{R} f_{*} \mathbb{R} \mathcal{H} o m_{\mathcal{D}_{Y}^{(m)}}\left(\mathcal{F}, f^{!} \mathcal{E}\right) \longleftarrow{ }_{\otimes \operatorname{oproj}_{f}} \mathbb{R} f_{*} \mathbb{R} \mathcal{H} o m_{\mathcal{D}_{Y}^{(m)}}\left(\mathcal{F}, f^{!} \mathcal{D}_{X}^{(m)}\right) \otimes_{\mathcal{D}_{X}^{(m)}}^{\mathbb{L}} \mathcal{E}
\end{aligned}
$$

est commutatif. 
Démonstration. - Par construction de $\delta_{f}$ (1.1.3.2), cela découle du fait que, pour tout $\mathcal{G} \in D\left(f^{-1} \mathcal{D}_{X}^{(m) \mathrm{d}}\right)$, le morphisme composé canonique : $\mathbb{R} f_{*} \mathcal{G} \otimes_{\mathcal{D}_{X}^{(m)}}^{\mathbb{L}}$ $\mathcal{D}_{X}^{(m)} \stackrel{\text { proj }_{f}}{\longrightarrow} \mathbb{R} f_{*}\left(\mathcal{G} \otimes_{f^{-1} \mathcal{D}_{X}^{(m)}}^{\mathbb{L}} f^{-1} \mathcal{D}_{X}^{(m)}\right) \stackrel{\sim}{\longrightarrow} \mathbb{R} f_{*} \mathcal{G}$ est l'isomorphisme canonique $\mathbb{R} f_{*} \mathcal{G} \otimes_{\mathcal{D}_{X}^{(m)}}^{\mathbb{L}} \mathcal{D}_{X}^{(m)} \stackrel{\sim}{\longrightarrow} \mathbb{R} f_{*} \mathcal{G}$.

Proposition 1.1.5. - Soient $\mathcal{E} \in D_{\mathrm{qc}, \mathrm{tdf}}^{\mathrm{b}}\left({ }^{\mathrm{g}} \mathcal{D}_{X}^{(m)}\right)$ et $\mathcal{G} \in D_{\mathrm{coh}}^{\mathrm{b}}\left({ }^{\mathrm{g}} \mathcal{D}_{Z}^{(m)}\right)$. Le diagramme suivant

$$
\begin{aligned}
& \mathbb{R H o m}_{\mathcal{D}_{X}^{(m)}}\left(f_{+} \circ g_{+}(\mathcal{G}), \mathcal{E}\right) \longleftarrow \sim \sim \mathbb{R} \mathcal{H o m}_{\mathcal{D}_{X}^{(m)}}\left((f \circ g)_{+}(\mathcal{G}), \mathcal{E}\right) \\
& \sim \downarrow \delta_{g} \circ \delta_{f} \quad \sim \downarrow \delta_{f \circ g} \\
& \mathbb{R} f_{*} \mathbb{R} g_{*} \mathbb{R} \mathcal{H} o m_{\mathcal{D}_{Z}^{(m)}}\left(\mathcal{G}, g^{!} f^{!} \mathcal{E}\right) \longleftarrow \sim \mathbb{R}(f \circ g)_{*} \mathbb{R} \mathcal{H} o m_{\mathcal{D}_{Z}^{(m)}}\left(\mathcal{G},(f \circ g)^{!} \mathcal{E}\right),
\end{aligned}
$$

où les isomorphismes horizontaux dérivent par fonctorialité des isomorphismes canoniques de composition de 1.1.2, est commutatif.

Démonstration. - Considérons le diagramme

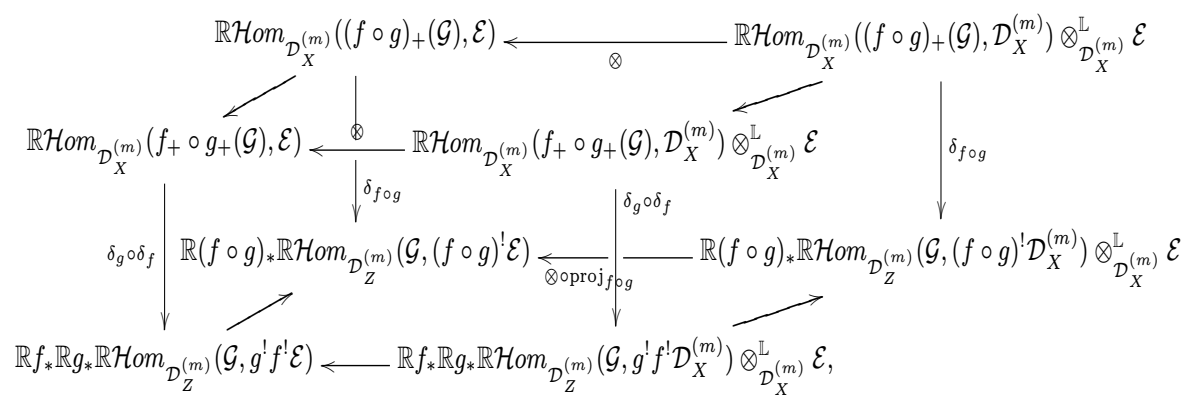

où le carré de gauche est 1.1.5.1 tandis celui de droite se déduit de 1.1.5.1 appliqué à $\mathcal{E}=\mathcal{D}_{X}^{(m)}$. Comme les flèches sont des isomorphismes, il suffit donc de vérifier que les carrés de droite, du haut, du fond et le composé de celui de devant avec celui du bas sont commutatifs.

D'après 1.1.4.1, le carré du fond est commutatif. Par fonctorialité, celui du haut l'est aussi. Afin de vérifier celle du carré de droite, nous aurons besoin du lemme suivant. 
Lemme 1.1.6. - Pour tout $\mathcal{G} \in D_{\mathrm{coh}}^{\mathrm{b}}\left({ }^{\mathrm{g}} \mathcal{D}_{Z}^{(m)}\right)$, le diagramme qui suit

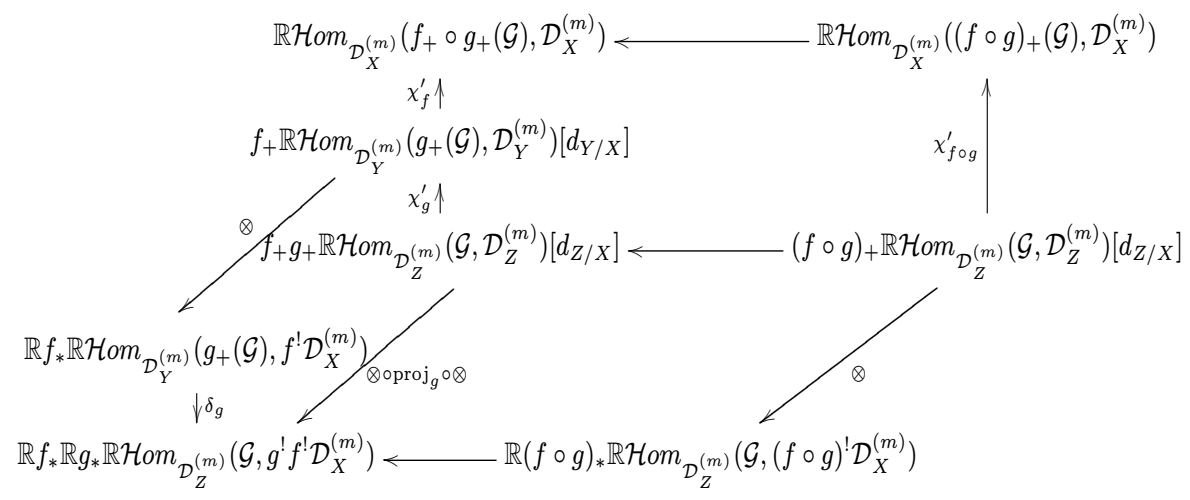

est commutatif.

Démonstration. - Le rectangle du fond est commutatif par transitivité en $f$ des isomorphismes $\chi_{f}^{\prime}$ (voir [6, 2.5.9]). Par construction de $\delta_{g}$ (voir 1.1.3), le diagramme

$$
\begin{aligned}
\mathbb{R} \mathcal{H o m}_{\mathcal{D}_{Y}^{(m)}}\left(g_{+}(\mathcal{G}), f^{!} \mathcal{D}_{X}^{(m)}\right) & \mathbb{R} \mathcal{H o m}_{\mathcal{D}_{Y}^{(m)}}\left(g_{+}(\mathcal{G}), \mathcal{D}_{Y}^{(m)}\right) \otimes_{\mathcal{D}_{Y}^{(m)}}^{\mathbb{L}} f^{!} \mathcal{D}_{X}^{(m)} \\
\downarrow^{\delta_{g}} & \chi_{g}^{\prime} \uparrow \\
\mathbb{R} g_{*} \mathbb{R} \mathcal{H o m}_{\mathcal{D}_{Z}^{(m)}}\left(\mathcal{G}, g^{!} f^{!} \mathcal{D}_{X}^{(m)}\right) & \\
\operatorname{Qoproj}_{g} \circ \otimes & g_{+} \mathcal{H o m}_{\mathcal{D}_{Y}^{(m)}}\left(\mathcal{G}, \mathcal{D}_{Y}^{(m)}\right) \otimes_{\mathcal{D}_{Y}^{(m)}}^{\mathbb{L}} f^{!} \mathcal{D}_{X}^{(m)}\left[d_{Z / Y}\right] .
\end{aligned}
$$

est commutatif. En appliquant $\mathbb{R} f_{*}$ à 1.1.6.1, comme $f_{+}(-)=\mathbb{R} f_{*}\left(-\otimes_{\mathcal{D}_{Y}^{(m)}}\right.$ $\left.f^{!}\left(\mathcal{D}_{X}^{(m)}\right)\right)\left[-d_{Y / X}\right]$, on obtient le carré de gauche de 1.1.6, qui est donc commutatif. Le carré du bas de 1.1.6 correspond (via 1.1.2.1) au contour de

$$
\begin{aligned}
& f_{+} g_{+} \mathbb{R} \mathcal{H} o m_{\mathcal{D}_{Z}^{(m)}}\left(\mathcal{G}, \mathcal{D}_{Z}^{(m)}\right)\left[d_{Z / X}\right]=\mathbb{R} f_{*}\left(\mathbb{R} g_{*}\left(\mathbb{R} \mathcal{H o m}_{\mathcal{D}_{Z}^{(m)}}\left(\mathcal{G}, \mathcal{D}_{Z}^{(m)}\right) \otimes_{\mathcal{D}_{Z}^{(m)}}^{\mathbb{L}} g^{!} \mathcal{D}_{Y}^{(m)}\right) \otimes_{\mathcal{D}_{Y}^{(m)}}^{\mathbb{L}} f^{!} \mathcal{D}_{X}^{(m)}\right) \\
& \downarrow \otimes \circ \operatorname{proj}_{g} \circ \otimes \quad \downarrow \operatorname{proj}_{g} \\
& \mathbb{R} f_{*} \mathbb{R} g_{*} \mathbb{R} \mathcal{H} o m_{\mathcal{D}_{Z}^{(m)}}\left(\mathcal{G}, g^{\prime} f^{!} \mathcal{D}_{X}^{(m)}\right) \longleftarrow \quad \mathbb{R} f_{*} \mathbb{R} g_{*}\left(\mathbb{R} \mathcal{H} o m_{\mathcal{D}_{Z}^{(m)}}\left(\mathcal{G}, \mathcal{D}_{Z}^{(m)}\right) \otimes_{\mathcal{D}_{Z}^{(m)}}^{\mathbb{L}} g^{!} f^{!} \mathcal{D}_{X}^{(m)}\right) \\
& \mathbb{R}(f \circ g)_{*} \mathbb{R} \mathcal{H} \circ m_{\mathcal{D}_{Z}^{(m)}}\left(\mathcal{G},(f \circ g)^{!} \mathcal{D}_{X}^{(m)}\right) \longleftarrow \otimes \mathbb{R}(f \circ g)_{*}\left(\mathbb{R} \mathcal{H} \circ m_{\mathcal{D}_{Z}^{(m)}}\left(\mathcal{G}, \mathcal{D}_{Z}^{(m)}\right) \otimes_{\mathcal{D}_{Z}^{(m)}}^{\mathbb{L}}(f \circ g)^{!} \mathcal{D}_{X}^{(m)}\right)
\end{aligned}
$$

BULletin DE LA SOCiÉtÉ MATHÉmATiQUe DE FRANCE 
Le carré du bas de 1.1.6.2 est commutatif par fonctorialité. On vérifie par fonctorialité ou transitivité la commutativité du diagramme suivant

$$
\begin{aligned}
& \mathbb{R} g_{*} \mathbb{R} \mathcal{H} m_{\mathcal{D}_{Z}^{(m)}}\left(\mathcal{G}, g^{\prime} \mathcal{D}_{Y}^{(m)}\right) \otimes_{\mathcal{D}_{Y}^{(m)}}^{\mathbb{L}} f^{\prime} \mathcal{D}_{X}^{(m)} \longleftarrow \mathbb{R g}_{*}\left(\mathbb{R} \mathcal{H} m_{\mathcal{D}_{Z}^{(m)}}\left(\mathcal{G}, \mathcal{D}_{Z}^{(m)}\right) \otimes_{\mathcal{D}_{Z}^{(m)}}^{\mathbb{L}} g^{\prime} \mathcal{D}_{Y}^{(m)}\right) \otimes_{\mathcal{D}_{Y}^{(m)}}^{\mathbb{L}} f^{!} \mathcal{D}_{X}^{(m)} \\
& { }^{\operatorname{proj}_{g}} \quad \operatorname{proj}_{g} \\
& \mathbb{R} g_{*}\left(\mathbb{R} \mathcal{H} o m_{\mathcal{D}_{Z}^{(m)}}\left(\mathcal{G}, g^{\prime} \mathcal{D}_{Y}^{(m)}\right) \otimes_{g^{-1} \mathcal{D}_{Y}^{(m)}}^{\mathbb{L}} g^{-1} f^{!} \mathcal{D}_{X}^{(m)}\right) \longleftarrow \underset{\otimes}{\mathbb{R} g_{*}\left(\mathbb{R} \mathcal{H} o m_{\mathcal{D}_{Z}^{(m)}}\left(\mathcal{G}, \mathcal{D}_{Z}^{(m)}\right) \otimes_{\mathcal{D}_{Z}^{(m)}}^{\mathbb{L}} g^{!} f^{!} \mathcal{D}_{X}^{(m)}\right)} \\
& \mathbb{R} g_{*} \mathbb{R} \mathcal{H}_{\mathcal{D}_{Z}^{(m)}}\left(\mathcal{G}, g^{\prime} \mathcal{D}_{Y}^{(m)} \otimes_{g^{-1} \mathcal{D}_{Y}^{(m)}}^{\mathbb{L}} g^{-1} f^{!} \mathcal{D}_{X}^{(m)}\right)=\sqrt{\otimes}=\sqrt{\mathbb{R} g_{*}\left(\mathbb{R} \mathcal{H} o m_{\mathcal{D}_{Z}^{(m)}}\left(\mathcal{G}, g^{!} f^{!} \mathcal{D}_{X}^{(m)}\right)\right),}
\end{aligned}
$$

dont les termes encadrés correspondent aux sommets du carré du haut de 1.1.6.2. En lui appliquant $\mathbb{R} f_{*}$, on obtient le carré du haut de 1.1.6.2.

Le foncteur $-\otimes_{\mathcal{D}_{X}^{(m)}}^{\mathbb{L}} \mathcal{E}$ appliqué au contour du diagramme de 1.1 .6 donne le carré de droite de 1.1.5.2, qui est donc commutatif.

Il reste à prouver que le carré de devant composé avec celui du bas de 1.1.5.2 est commutatif. Pour cela considérons le diagramme

$$
\begin{aligned}
& \mathbb{R} \mathcal{H} o m_{\mathcal{D}_{X}^{(m)}}\left(f_{+} \circ g_{+}(\mathcal{G}), \mathcal{E}\right) \longleftarrow \quad \mathbb{R} \mathcal{H} m_{\mathcal{D}_{X}^{(m)}}\left(f_{+} \circ g_{+}(\mathcal{G}), \mathcal{D}_{X}^{(m)}\right) \otimes_{\mathcal{D}_{X}^{(m)}}^{\mathbb{L}} \mathcal{E} \\
& \gamma_{f} \quad \forall \delta_{f} \\
& \mathbb{R} f_{*} \mathbb{R} \mathcal{H} m_{\mathcal{D}_{Y}^{(m)}}\left(g_{+}(\mathcal{G}), f^{!} \mathcal{E}\right) \longleftarrow \otimes_{\otimes \operatorname{proj}_{f}} \mathbb{R} f_{*} \mathbb{R} \mathcal{H} o m_{\mathcal{D}_{Y}^{(m)}}\left(g_{+}(\mathcal{G}), f^{!} \mathcal{D}_{X}^{(m)}\right) \otimes_{\mathcal{D}_{X}^{(m)}}^{\mathbb{L}} \mathcal{E} \\
& \otimes \uparrow \quad \| \\
& \mathbb{R} f_{*}\left(\mathbb{R} \mathcal{H} o m_{\mathcal{D}_{Y}^{(m)}}\left(g_{+}(\mathcal{G}), f^{!} \mathcal{D}_{X}^{(m)}\right) \otimes_{f^{-1} \mathcal{D}_{X}^{(m)}}^{\mathbb{L}} f^{-1} \mathcal{E}\right) \longleftarrow_{\operatorname{proj}_{f}} \mathbb{R} f_{*} \mathbb{R} \mathcal{H} o m_{\mathcal{D}_{Y}^{(m)}}\left(g_{+}(\mathcal{G}), f^{\prime} \mathcal{D}_{X}^{(m)}\right) \otimes_{\mathcal{D}_{X}^{(m)}}^{\mathbb{L}} \mathcal{E} \\
& \otimes \uparrow \quad \otimes \uparrow \\
& \mathbb{R} f_{*}\left(\mathbb{R} \mathcal{H} o m_{\mathcal{D}_{Y}^{(m)}}\left(g_{+}(\mathcal{G}), \mathcal{D}_{Y}^{(m)}\right) \otimes_{\mathcal{D}_{Y}^{(m)}}^{\mathbb{L}} f^{\prime} \mathcal{E}\right) \underset{\operatorname{proj}_{f}}{\longleftarrow} \mathbb{R} f_{*}\left(\mathbb{R} \mathcal{H} o m_{\mathcal{D}_{Y}^{(m)}}\left(g_{+}(\mathcal{G}), \mathcal{D}_{Y}^{(m)}\right) \otimes_{\mathcal{D}_{Y}^{(m)}}^{\mathbb{L}} f^{!} \mathcal{D}_{X}^{(m)}\right) \otimes_{\mathcal{D}_{X}^{(m)}}^{\mathbb{L}} \mathcal{E} \\
& \psi \delta_{g} \quad \forall \delta_{g} \\
& \mathbb{R} f_{*}\left(\mathbb{R} g_{*} \mathbb{R} \mathcal{H} o m_{\mathcal{D}_{Z}^{(m)}}\left(\mathcal{G}, g^{\prime} \mathcal{D}_{Y}^{(m)}\right) \otimes_{\mathcal{D}_{Y}^{(m)}}^{\mathbb{L}} f^{!} \mathcal{E}\right) \underset{\operatorname{proj}_{f}}{\leftarrow} \mathbb{R} f_{*}\left(\mathbb{R} g_{*} \mathbb{R} \mathcal{H} o m_{\mathcal{D}_{Z}^{(m)}}\left(\mathcal{G}, g^{\prime} \mathcal{D}_{Y}^{(m)}\right) \otimes_{\mathcal{D}_{Y}^{(m)}}^{\mathbb{L}} f^{!} \mathcal{D}_{X}^{(m)}\right) \otimes_{\mathcal{D}_{X}^{(m)}}^{\mathbb{L}} \mathcal{E} \\
& \downarrow \otimes \operatorname{oproj}_{g} \quad \forall \otimes \circ \operatorname{proj}_{g} \\
& \mathbb{R} f_{*} \mathbb{R} g_{*} \mathbb{R} \mathcal{H} m_{\mathcal{D}_{Z}^{(m)}}\left(\mathcal{G}, g^{!} f^{!} \mathcal{E}\right) \\
& \text { can } \uparrow \quad \operatorname{can} \uparrow
\end{aligned}
$$

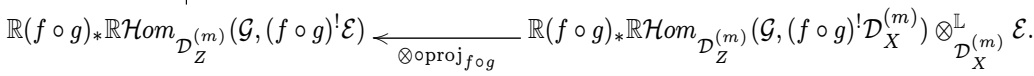

Grâce à 1.1.4, la flèche composée de gauche de 1.1.6.3 :

$$
\mathbb{R} f_{*} \mathbb{R} \mathcal{H} o m_{\mathcal{D}_{Y}^{(m)}}\left(g_{+}(\mathcal{G}), f^{!} \mathcal{E}\right) \rightarrow \mathbb{R} f_{*} \mathbb{R} g_{*} \mathbb{R} \mathcal{H} o m_{\mathcal{D}_{Z}^{(m)}}\left(\mathcal{G}, g^{!} f^{!} \mathcal{E}\right)
$$

est celle induite par $\delta_{g}$ (plus précisément $\mathbb{R} f_{*} \delta_{g}$ ). De manière analogue, le composé de droite 1.1.6.3:

$$
\begin{aligned}
& \mathbb{R} f_{*} \mathbb{R} \mathcal{H o m}{ }_{\mathcal{D}_{Y}^{(m)}}\left(g_{+}(\mathcal{G}), f^{!} \mathcal{D}_{X}^{(m)}\right) \otimes_{\mathcal{D}_{X}^{(m)}}^{\mathbb{L}} \mathcal{E} \rightarrow \mathbb{R} f_{*} \mathbb{R} g_{*} \mathbb{R} \mathcal{H} o m_{\mathcal{D}_{Z}^{(m)}}\left(\mathcal{G}, g^{!} f^{!} \mathcal{D}_{X}^{(m)}\right) \otimes_{\mathcal{D}_{X}^{(m)}}^{\mathbb{L}} \mathcal{E} \\
& \quad \text { томе } 137-2009-\mathrm{N}^{\circ} 4
\end{aligned}
$$


se déduit fonctoriellement de $\delta_{g}$ (est égale à $\mathbb{R} f_{*} \delta_{g} \otimes \mathrm{Id}$. Il en résulte que la commutativité du carré de devant composé avec celui du bas de 1.1.5.2 est équivalente à celle de 1.1.6.3. Établissons alors cette dernière.

D'après 1.1.4.1, le carré du haut de 1.1.6.3 est commutatif. Par fonctorialité ou définition, il en est de même des trois autres. Il reste à vérifier la commutativité du rectangle. Pour cela considérons le diagramme suivant.

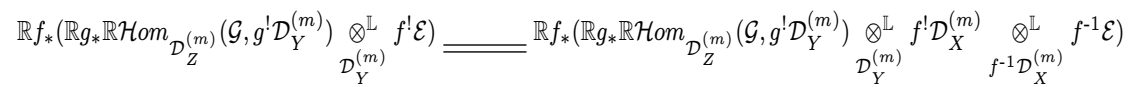

$$
\begin{aligned}
& \downarrow \operatorname{proj}_{g} \quad \forall \operatorname{proj}_{g}
\end{aligned}
$$

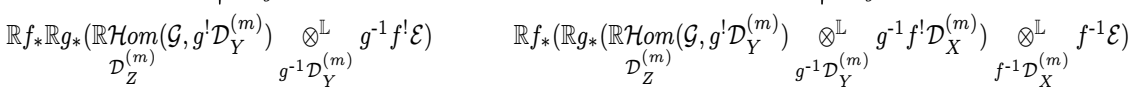

$$
\begin{aligned}
& \downarrow \otimes
\end{aligned}
$$

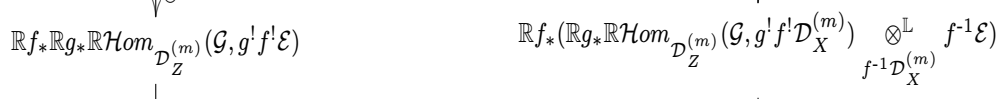

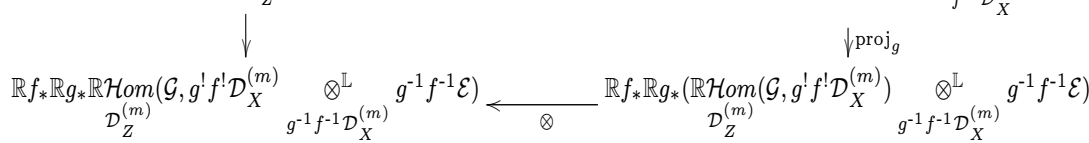

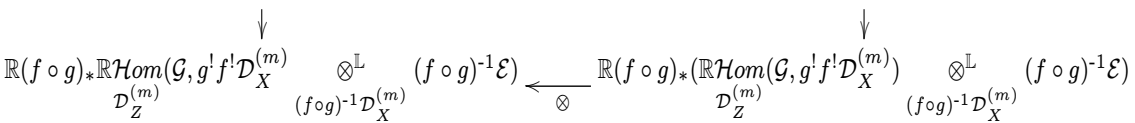

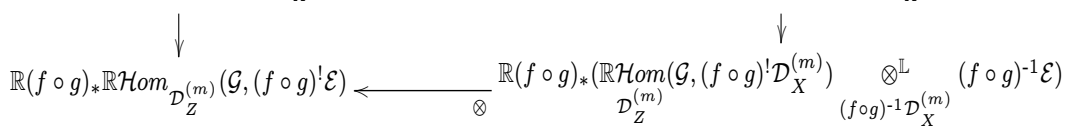

Les deux carrés de 1.1.6.4 sont commutatifs par fonctorialité. Nous aurons besoin des deux lemmes ci-après, dont les preuves sont aisées, qui établissent deux conditions de transitivité validées par les morphismes de projection.

Lemme 1.1.7. - Soient $\mathcal{H} \in D\left(g^{-1} \mathcal{D}_{Y}^{(m) \mathrm{d}}\right), \mathcal{E}_{1} \in D_{(\mathrm{tdf}, \mathrm{qc}, \cdot)}\left({ }^{g} \mathcal{D}_{Y}^{(m)}, f^{-1} \mathcal{D}_{X}^{(m) \mathrm{d}}\right)$ et $\mathcal{E}_{2} \in D_{\mathrm{tdf}, \mathrm{qc}}\left(f^{-1} \mathcal{D}_{X}^{(m) \mathrm{d}}\right)$. Le diagramme

$$
\begin{aligned}
& \mathbb{R} g_{*} \mathcal{H} \otimes_{\mathcal{D}_{Y}^{(m)}}^{\mathbb{L}} \mathcal{E}_{1} \otimes_{f^{-1} \mathcal{D}_{X}^{(m)}}^{\mathbb{L}} \mathcal{E}_{2} \underset{\operatorname{proj}_{g}}{\sim} \mathbb{R} g_{*}\left(\mathcal{H} \otimes_{g^{-1} \mathcal{D}_{Y}^{(m)}}^{\mathbb{L}} g^{-1} \mathcal{E}_{1}\right) \otimes_{f^{-1} \mathcal{D}_{X}^{(m)}}^{\mathbb{L}} \mathcal{E}_{2} \\
& \sim \downarrow \operatorname{proj}_{g} \quad \sim \downarrow \operatorname{proj}_{g} \\
& \mathbb{R} g_{*} \mathcal{H} \otimes_{g^{-1} \mathcal{D}_{Y}^{(m)}}^{\mathbb{L}} g^{-1}\left(\mathcal{E}_{1} \otimes_{f^{-1} \mathcal{D}_{X}^{(m)}}^{\mathbb{L}} \mathcal{E}_{2}\right) \longrightarrow \underset{\sim}{\longrightarrow} g_{*}\left(\mathcal{H} \otimes_{g^{-1} \mathcal{D}_{Y}^{(m)}}^{\mathbb{L}} g^{-1} \mathcal{E}_{1} \otimes_{g^{-1} f^{-1} \mathcal{D}_{X}^{(m)}}^{\mathbb{L}} g^{-1} \mathcal{E}_{2}\right)
\end{aligned}
$$

est commutatif. 
LEMme 1.1.8. - Soient $\mathcal{H} \in D\left(g^{-1} f^{-1} \mathcal{D}_{X}^{(m) \mathrm{d}}\right)$ et $\mathcal{E} \in D_{\mathrm{qc}, \mathrm{tdf}}^{\mathrm{b}}\left({ }^{\mathrm{g}} \mathcal{D}_{X}^{(m)}\right)$. Le diagramme

$$
\begin{gathered}
\mathbb{R} f_{*}\left(\mathbb{R} g_{*} \mathcal{H} \otimes_{f^{-1} \mathcal{D}_{X}^{(m)}}^{\mathbb{L}} f^{-1} \mathcal{E}\right) \leftarrow \underset{\operatorname{proj}_{f}}{\downarrow} \mathbb{R} f_{*} \mathbb{R} g_{*} \mathcal{H} \otimes_{\mathcal{D}_{X}^{(m)}}^{\mathbb{L}} \mathcal{E} \\
\mathbb{R} f_{*} \mathbb{R} g_{*}\left(\mathcal{H} \otimes_{g^{-1} f^{-1} \mathcal{D}_{X}^{(m)}}^{\mathbb{L}} g^{-1} f^{-1} \mathcal{E}\right)_{\operatorname{proj}_{f \circ g}}^{\leftarrow} \mathbb{R}(f \circ g)_{*}\left(\mathcal{H} \otimes_{(f \circ g)^{-1} \mathcal{D}_{X}^{(m)}}^{\mathbb{L}}(f \circ g)^{-1} \mathcal{E}\right)
\end{gathered}
$$

est commutatif.

En utilisant le lemme 1.1.7, la transitivité des isomorphismes de la forme $\otimes$, on vérifie la commutativité du rectangle (en haut) de 1.1.6.4 où l'on a omis $\mathbb{R} f_{*}$. Le diagramme 1.1.6.4 est donc commutatif.

On parvient par fonctorialité de 1.1 .8 à la commutativité de rectangle du milieu de :

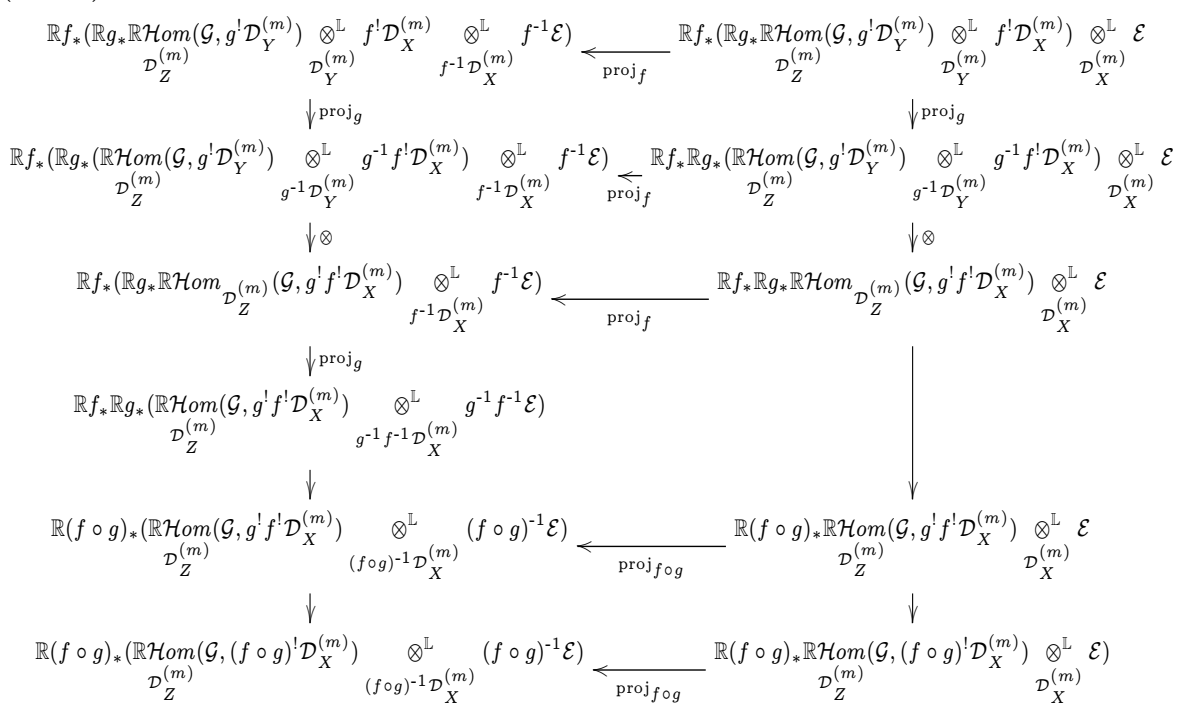

On obtient des carrés par fonctorialité. Ainsi, 1.1.8.1 est commutatif.

Le composé de 1.1.6.4 et 1.1.8.1 correspond au rectangle de 1.1.6.3. Celuici est donc commutatif. On a donc vérifié la commutativité du diagramme 1.1.6.3, i.e., celle du carré de devant composé avec celui du bas de 1.1.5.2. D'où le résultat. 
1.1.9. - Soient $\mathcal{E} \in D_{\mathrm{qc}, \mathrm{tdf}}^{\mathrm{b}}\left({ }^{\mathrm{g}} \mathcal{D}_{X}^{(m)}\right)$ et $\mathcal{F} \in D_{\text {coh }}^{\mathrm{b}}\left({ }^{\mathrm{g}} \mathcal{D}_{Y}^{(m)}\right)$. En appliquant $\mathbb{R} \Gamma(X,-)$ et $\mathrm{H}^{0} \circ \mathbb{R} \Gamma(X,-)$ à $\delta_{f}$, on obtient les bijections :

$$
\begin{aligned}
\operatorname{adj}_{f, \mathcal{F}, \mathcal{E}}: \mathbb{R H o m}_{\mathcal{D}_{X}^{(m)}}\left(f_{+}(\mathcal{F}), \mathcal{E}\right) \stackrel{\sim}{\longrightarrow} \mathbb{R H o m}_{\mathcal{D}_{Y}^{(m)}}\left(\mathcal{F}, f^{!} \mathcal{E}\right), \\
\operatorname{adj}_{f, \mathcal{F}, \mathcal{E}}: \operatorname{Hom}_{\mathcal{D}_{X}^{(m)}}\left(f_{+}(\mathcal{F}), \mathcal{E}\right) \stackrel{\sim}{\longrightarrow} \operatorname{Hom}_{\mathcal{D}_{Y}^{(m)}}\left(\mathcal{F}, f^{!} \mathcal{E}\right) .
\end{aligned}
$$

Si aucune confusion n'est à craindre, on les notera $\operatorname{adj}_{f}$.

Il découle immédiatement de 1.1 .5 le corollaire qui suit.

Corollaire 1.1.10. - Soient $\mathcal{E} \in D_{\mathrm{qc}, \mathrm{tdf}}^{\mathrm{b}}\left({ }^{\mathrm{g}} \mathcal{D}_{X}^{(m)}\right)$ et $\mathcal{G} \in D_{\mathrm{coh}}^{\mathrm{b}}\left({ }^{\mathrm{g}} \mathcal{D}_{Z}^{(m)}\right)$. Le diagramme

$$
\begin{aligned}
& \mathbb{R H o m}_{\mathcal{D}_{X}^{(m)}}\left(f_{+} \circ g_{+}(\mathcal{G}), \mathcal{E}\right) \longleftarrow \mathbb{R H o m}_{\mathcal{D}_{X}^{(m)}}\left((f \circ g)_{+}(\mathcal{G}), \mathcal{E}\right) \\
& \sim \vee \operatorname{adj}_{g} \circ \operatorname{adj}_{f} \quad \sim \vee \operatorname{adj}_{f \circ g} \\
& \mathbb{R H o m}_{\mathcal{D}_{Z}^{(m)}}\left(\mathcal{G}, g^{!} f^{!} \mathcal{E}\right) \longleftarrow \sim\left(\mathbb{R} \operatorname{Hom}_{\mathcal{D}_{Z}^{(m)}}\left(\mathcal{G},(f \circ g)^{!} \mathcal{E}\right)\right.
\end{aligned}
$$

est commutatif. De même en enlevant « $\mathbb{R} »$.

1.1.11. - Soit $\mathcal{F} \in D_{\text {coh }}^{\mathrm{b}}\left({ }^{\mathrm{g}} \mathcal{D}_{Y}^{(m)}\right)$. L'image de l'identité de $f_{+}(\mathcal{F})$ par la bijection $\operatorname{adj}_{f, \mathcal{F}, f_{+}(\mathcal{F})}$ de 1.1 .9 donne le morphisme $\mathcal{F} \rightarrow f^{!} f_{+}(\mathcal{F})$ noté $\operatorname{adj}_{f, \mathcal{F}}$ ou simplement $\operatorname{adj}_{\mathcal{F}}$ ou $\operatorname{adj}_{f}$.

Soit $\mathcal{E} \in D_{\text {coh }}^{\mathrm{b}}\left({ }^{\mathrm{g}} \mathcal{D}_{X}^{(m)}\right)$ tel que $f^{!} \mathcal{E} \in D_{\text {coh }}^{\mathrm{b}}\left({ }^{\mathrm{g}} \mathcal{D}_{Y}^{(m)}\right)$. On notera $\operatorname{adj}_{f, \mathcal{E}}: f_{+} \circ$

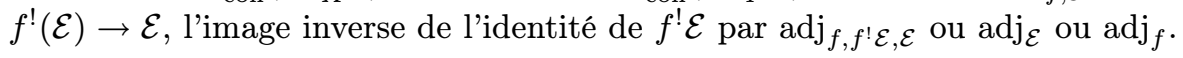

Proposition 1.1.12. - Pour tout $\mathcal{G} \in D_{\mathrm{coh}}^{\mathrm{b}}\left({ }^{\mathrm{g}} \mathcal{D}_{Z}^{(m)}\right)$ et pour tout $\mathcal{E} \in$ $D_{\text {coh }}^{\mathrm{b}}\left({ }^{\mathrm{g}} \mathcal{D}_{X}^{(m)}\right)$ tel que $f^{!}(\mathcal{E}) \in D_{\text {coh }}^{\mathrm{b}}\left({ }^{\mathrm{g}} \mathcal{D}_{Y}^{(m)}\right)$ et $(f \circ g)^{!}(\mathcal{E}) \in D_{\text {coh }}^{\mathrm{b}}\left({ }^{\mathrm{g}} \mathcal{D}_{Z}^{(m)}\right)$, les diagrammes

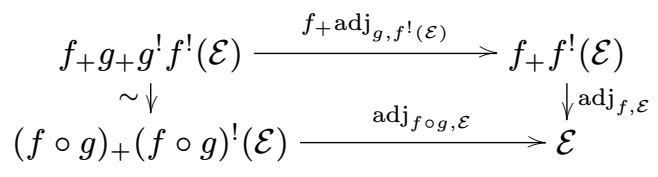

et

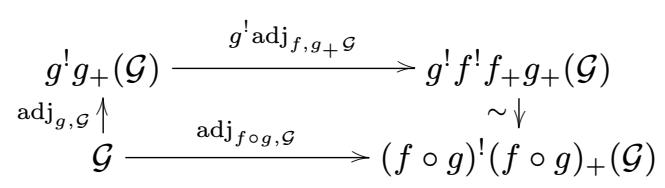

sont commutatifs. 
Démonstration. - Notons «can » les isomorphismes (ou induit fonctoriellement par ceux $)$ de la forme $g^{!} f^{!} \stackrel{\sim}{\longrightarrow}(f \circ g)^{!}$ou $f_{+} g_{+} \stackrel{\sim}{\longrightarrow}(f \circ g)_{+}$. La démonstration de la commutativité du diagramme de droite étant analogue, contentons-nous de vérifier celle de gauche. À cette fin, considérons le diagramme ci-après :

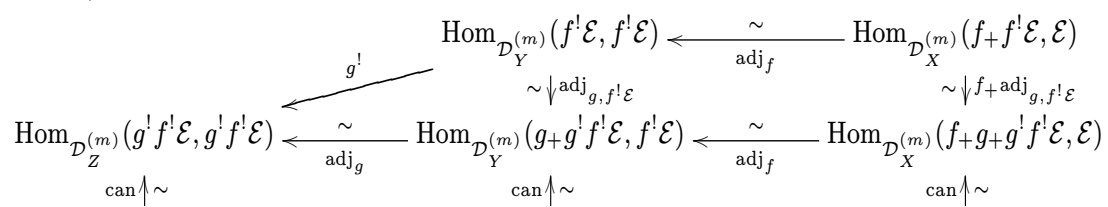

$$
\begin{aligned}
& \operatorname{Hom}_{\mathcal{D}_{Z}^{(m)}}\left((f \circ g)^{!} \mathcal{E}, g^{!} f^{!} \mathcal{E}\right) \underset{\operatorname{adj}_{g}}{\sim} \operatorname{Hom}_{\mathcal{D}_{Y}^{(m)}}\left(g_{+}(f \circ g)^{!} \mathcal{E}, f^{\prime} \mathcal{E}\right) \underset{\operatorname{adj}_{f}}{\sim} \operatorname{Hom}_{\mathcal{D}_{X}^{(m)}}\left(f_{+} g_{+}(f \circ g)^{!} \mathcal{E}, \mathcal{E}\right) \\
& \operatorname{can} \uparrow \sim \quad \operatorname{can} \uparrow \sim \\
& \operatorname{Hom}_{\mathcal{D}_{Z}^{(m)}}\left((f \circ g)^{!} \mathcal{E},(f \circ g)^{!} \mathcal{E}\right) \longleftarrow \underset{\operatorname{adj}_{f \circ g}}{\sim} \operatorname{Hom}_{\mathcal{D}_{X}^{(m)}}\left((f \circ g)_{+}(f \circ g)^{!} \mathcal{E}, \mathcal{E}\right),
\end{aligned}
$$

dont les bijections horizontales sont les isomorphismes d'adjonction de 1.1.9.2. Il dérive de 1.1.10 la commutativité du rectangle du bas de 1.1.12.1. Par fonctorialité en le terme de droite $f^{\prime} \mathcal{E}$, il suffit de vérifier celle du triangle pour l'identité de $f^{\prime} \mathcal{E}$, ce qui est immédiat. Enfin, celle des carrés s'établissent par fonctorialité. Le diagramme 1.1.12.1 est donc commutatif.

Or, la flèche $\operatorname{Hom}_{\mathcal{D}_{X}^{(m)}}\left(f_{+} g_{+} g^{!} f^{!} \mathcal{E}, \mathcal{E}\right) \rightarrow \operatorname{Hom}_{\mathcal{D}_{Z}^{(m)}}\left((f \circ g)^{!} \mathcal{E},(f \circ g)^{!} \mathcal{E}\right)$ de 1.1.12.1 passant par le droite puis par le bas (resp. par le haut puis par la gauche), envoie $\operatorname{adj}_{f \circ g, \mathcal{E}} \circ$ can $\left(\operatorname{resp} . \operatorname{adj}_{f, \mathcal{E}} \circ\left(f_{+} \operatorname{adj}_{g, f^{!}(\mathcal{E})}\right)\right)$ sur l'identité.

\subsection{Cas des schémas formels}

1.2.1. - Soient $\left.\mathcal{E}, \mathcal{F} \in D_{\mathrm{qc}}^{\mathrm{b}}{ }^{\mathrm{g}} \widehat{\mathcal{D}}_{\mathfrak{X}}^{(m)}\right)$. Pour tout entier $i \geq 0$, on pose $\mathcal{E}_{i}:=$ $\mathcal{O}_{S_{i}} \otimes_{\mathcal{O}_{\mathcal{S}}}^{\mathbb{L}} \mathcal{\sim} \stackrel{\sim}{\longrightarrow} \mathcal{D}_{X_{i}}^{(m)} \otimes_{\widehat{\mathcal{D}}_{\mathfrak{X}}^{(m)}}^{\mathbb{L}} \mathcal{E}$ (de même pour $\mathcal{F}$ ). On dispose de l'isomorphisme de changement de base suivant :

$(1.2 .1 .1)$

$$
\mathcal{O}_{S_{i}} \otimes_{\mathcal{O}_{\mathcal{S}}}^{\mathbb{L}} \mathbb{R} \mathcal{H} o m_{\widehat{\mathcal{D}}_{\mathfrak{X}}^{(m)}}(\mathcal{E}, \mathcal{F}) \stackrel{\sim}{\longrightarrow} \mathbb{R} \mathcal{H o m}_{\widehat{\mathcal{D}}_{\mathfrak{X}}^{(m)}}\left(\mathcal{E}, \mathcal{F}_{i}\right) \stackrel{\sim}{\sim} \mathcal{H}_{o m_{\mathcal{D}_{X_{i}}^{(m)}}}\left(\mathcal{E}_{i}, \mathcal{F}_{i}\right)
$$

Celui-ci est transitif en $i$, i.e., pour tout $1 \leq i^{\prime} \leq i$, on construit de manière analogue des isomorphismes $\mathcal{O}_{S_{i^{\prime}}} \otimes_{\mathcal{O}_{S_{i}}}^{\mathbb{L}} \mathbb{R} \mathcal{H o m}_{\mathcal{D}_{X_{i}}^{(m)}}\left(\mathcal{E}_{i}, \mathcal{F}_{i}\right) \stackrel{\sim}{\longrightarrow} \mathbb{R} \mathcal{H o m}_{\mathcal{D}_{X_{i^{\prime}}}^{(m)}}\left(\mathcal{E}_{i^{\prime}}, \mathcal{F}_{i^{\prime}}\right)$, ceux-ci étant compatibles avec 1.2.1.1.

On remarque enfin que le composé

$$
\mathbb{R H o m}_{\widehat{\mathcal{D}}_{\mathfrak{X}}^{(m)}}(\mathcal{E}, \mathcal{F}) \rightarrow \mathcal{O}_{S_{i}} \otimes_{\mathcal{O}_{\mathcal{S}}}^{\mathbb{L}} \mathbb{R} \mathcal{H} o m_{\widehat{\mathcal{D}}_{\mathfrak{X}}^{(m)}}(\mathcal{E}, \mathcal{F}) \stackrel{\sim}{\longrightarrow} \mathbb{R} \mathcal{H} o m_{\mathcal{D}_{X_{i}}^{(m)}}\left(\mathcal{E}_{i}, \mathcal{F}_{i}\right),
$$

dont l'isomorphisme est 1.2.1.1, est le morphisme canonique $\mathcal{O}_{S_{i}} \otimes_{\mathcal{O}_{\mathcal{S}}}^{\mathbb{L}}-$ : $\mathbb{R}_{\mathcal{H} o m_{\widehat{\mathcal{D}}_{\mathfrak{X}}^{(m)}}}(\mathcal{E}, \mathcal{F}) \rightarrow \mathbb{R} \mathcal{H o m}_{\mathcal{D}_{X_{i}}^{(m)}}\left(\mathcal{E}_{i}, \mathcal{F}_{i}\right)$ 
1.2.2. - Soient $f: \mathcal{Y} \rightarrow \mathfrak{X}$ un morphisme propre de $\mathcal{V}$-schémas formels lisses, $\mathcal{E} \in D_{\mathrm{qc}}^{\mathrm{b}}\left({ }^{\mathrm{g}} \widehat{\mathcal{D}}_{\mathfrak{X}}^{(m)}\right)$. Par construction de l'image inverse extraordinaire définie pour les complexes quasi-cohérents (voir [6, 3.4.2.1] et aussi [6, 3.2.2]), on dispose de l'isomorphisme de changement de base de l'image inverse extraordinaire $\mathcal{O}_{S_{i}} \otimes_{\mathcal{O}_{\mathcal{S}}}^{\mathbb{L}} f^{!}(\mathcal{E}) \stackrel{\sim}{\longrightarrow} f_{i}^{!}\left(\mathcal{E}_{i}\right)$. Lorsque $\left.\mathcal{E} \in D_{\text {coh }}^{\mathrm{b}}{ }^{\mathrm{g}} \widehat{\mathcal{D}}_{\mathfrak{X}}^{(m)}\right)$, on identifiera $f^{!}(\mathcal{E})$ avec $\widehat{\mathcal{D}}_{\mathcal{Y} \rightarrow \mathfrak{X}}^{(m)} \otimes_{f^{-1} \widehat{\mathcal{D}}_{\mathfrak{X}}^{(m)}}^{\mathbb{L}} f^{-1} \mathcal{E}\left[d_{Y / X}\right]$. On bénéficie alors, pour tout $\mathcal{E} \in D_{\text {coh }}^{\mathrm{b}}\left({ }^{\mathrm{g}} \widehat{\mathcal{D}}_{\mathfrak{X}}^{(m)}\right)$, de l'isomorphisme de changement de base via les isomorphismes : $(1.2 .2 .1)$

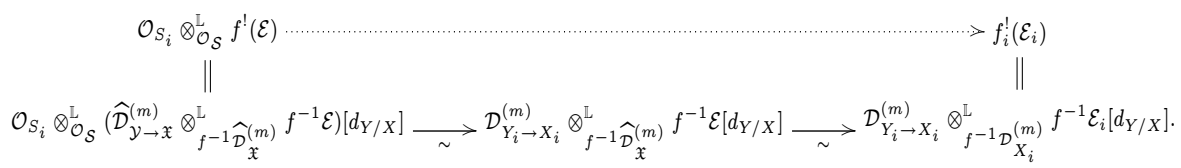

Comme pour 1.2.1.1, celui-ci est transitif en $i$, i.e., on en tire un isomorphisme entre les systèmes projectifs $\left(\mathcal{O}_{S_{i}} \otimes_{\mathcal{O}_{\mathcal{S}}}^{\mathbb{L}} f^{!}(\mathcal{E})\right)_{i \in \mathbb{N}}$ et $\left(f_{i}^{!}\left(\mathcal{E}_{i}\right)\right)_{i \in \mathbb{N}}$.

De plus, pour tout $\mathcal{M} \in D_{\mathrm{qc}}^{\mathrm{b}}\left(\widehat{\mathcal{D}}_{\mathcal{Y}}^{(m) \mathrm{d}}\right)$, on dispose tautologiquement de l'isomorphisme de changement de base de l'image directe (voir [6, 3.5.1.1]) : $f_{+}^{\mathrm{d}}(\mathcal{M}) \otimes_{\mathcal{O}_{\mathcal{S}}}^{\mathbb{L}} \mathcal{O}_{S_{i}} \stackrel{\sim}{\longrightarrow} f_{i+}^{\mathrm{d}}\left(\mathcal{M}_{i}\right)$. De même, lorsque $\mathcal{M} \in D_{\text {coh }}^{\mathrm{b}}\left(\widehat{\mathcal{D}}_{\mathcal{Y}}^{(m) \mathrm{d}}\right)$, celui-ci est défini via le diagramme commutatif :

$$
\begin{gathered}
f_{+}^{\mathrm{d}}(\mathcal{M}) \otimes_{\mathcal{O}_{\mathcal{S}}}^{\mathbb{L}} \mathcal{O}_{S_{i}}=\left(\mathbb{R} f_{*}\left(\mathcal{M} \otimes_{\widehat{\mathcal{D}}_{\mathcal{Y}}^{(m)}}^{\mathbb{L}} \widehat{\mathcal{D}}_{\mathcal{Y} \rightarrow \mathfrak{X}}^{(m)}\right)\right) \otimes_{\mathcal{O}_{\mathcal{S}}}^{\mathbb{L}} \mathcal{O}_{S_{i}} \underset{\sim \operatorname{proj}}{\longrightarrow} \mathbb{R} f_{*}\left(\mathcal{M} \otimes_{\widehat{\mathcal{D}}_{\mathcal{Y}}^{(m)}}^{\mathbb{L}} \widehat{\mathcal{D}}_{\mathcal{Y} \rightarrow \mathfrak{X}}^{(m)} \otimes_{\mathcal{O}_{\mathcal{S}}}^{\mathbb{L}} \mathcal{O}_{S_{i}}\right) \\
\sim \vdots \\
f_{i+}^{\mathrm{d}}\left(\mathcal{M}_{i}\right)= \\
=
\end{gathered}
$$

Comme pour 1.2.1.1, l'isomorphisme de gauche de 1.2.2.2 est transitif en $i$, i.e., induit un isomorphisme entre les systèmes projectifs $\left(f_{+}^{\mathrm{d}}(\mathcal{M}) \otimes_{\mathcal{O}_{\mathcal{S}}}^{\mathbb{L}} \mathcal{O}_{S_{i}}\right)_{i \in \mathbb{N}}$ et $\left(f_{i+}^{\mathrm{d}}\left(\mathcal{M}_{i}\right)\right)_{i \in \mathbb{N}}$. De même, en remplaçant dans 1.2.2.2 le symbole « d » par « $\mathrm{g}$ ».

1.2.3. - Soient $f: \mathcal{Y} \rightarrow \mathfrak{X}$ un morphisme propre de $\mathcal{V}$-schémas formels lisses, $\mathcal{E} \in D_{\text {coh }}^{\mathrm{b}}\left(\widehat{\mathcal{D}}_{\mathfrak{X}}^{(m)}\right)$ et $\mathcal{F} \in D_{\text {coh }}^{\mathrm{b}}\left(\widehat{\mathcal{D}}_{\mathcal{Y}}^{(m)}\right)$. Comme pour 1.1.3.1, on dispose d'un isomorphisme canonique

$$
\chi_{f}^{\prime}: f_{+}^{\mathrm{d}} \mathbb{R} \mathcal{H} o m_{\widehat{\mathcal{D}}_{\mathcal{Y}}^{(m)}}\left(\mathcal{F}, \widehat{\mathcal{D}}_{\mathcal{Y}}^{(m)}\right)\left[d_{Y}\right] \stackrel{\sim}{\longrightarrow} \mathbb{R} \mathcal{H} m_{\widehat{\mathcal{D}}_{\mathfrak{X}}^{(m)}}\left(f_{+}^{\mathrm{g}}(\mathcal{F}), \widehat{\mathcal{D}}_{\mathfrak{X}}^{(m)}\right)\left[d_{X}\right]
$$

Ce dernier s'inscrit dans le diagramme commutatif ci-après :

$$
\begin{aligned}
& \mathcal{O}_{S_{i}} \otimes_{\mathcal{O}_{\mathcal{S}}}^{\mathbb{L}}\left(f_{+}^{\mathrm{d}} \mathbb{R} \mathcal{H}_{\widehat{D}^{(m)}}\left(\mathcal{F}, \widehat{\mathcal{D}}_{\mathcal{Y}}^{(m)}\right)\left[d_{Y}\right]\right) \stackrel{\chi_{f}^{\prime}}{\sim} \mathcal{O}_{S_{i}} \otimes_{\mathcal{O}_{\mathcal{S}}}^{\mathbb{L}}\left(\mathbb{R} \mathcal{H}_{\widehat{\mathcal{D}}^{(m)}}\left(f_{+}^{\mathrm{g}}(\mathcal{F}), \widehat{\mathcal{D}}_{\mathfrak{X}}^{(m)}\right)\left[d_{X}\right]\right) \\
& \sim \downarrow \quad \sim \downarrow \\
& f_{i+}^{\mathrm{d}} \mathbb{R} \mathcal{H o m}_{\mathcal{D}_{Y_{i}}^{(m)}}\left(\mathcal{F}_{i}, \mathcal{D}_{Y_{i}}^{(m)}\right)\left[d_{Y}\right] \underset{\sim}{\chi_{f}^{\prime}} \mathbb{R} \mathcal{H o m}_{\mathcal{D}_{X_{i}}^{(m)}}\left(f_{i+}^{\mathrm{g}}\left(\mathcal{F}_{i}\right), \mathcal{D}_{X_{i}}^{(m)}\right)\left[d_{X}\right],
\end{aligned}
$$


dont les isomorphisme verticaux sont induits par les isomorphismes de changement de base de 1.2.1 et 1.2.2.

De façon analogue à 1.1.3.3 (pour le morphisme de projection, nous utilisons la cohérence de $\mathcal{E}$ ), on construit grâce à $\chi_{f}^{\prime}$ l'isomorphisme :

$$
\delta_{f}: \mathbb{R}_{\mathcal{H o m}_{\widehat{\mathcal{D}}_{X}^{(m)}}}\left(f_{+}(\mathcal{F}), \mathcal{E}\right) \stackrel{\sim}{\longrightarrow} \mathbb{R} f_{*} \mathbb{R} \mathcal{H} \text { om }{\widehat{\mathcal{D}}_{Y}^{(m)}}\left(\mathcal{F}, f^{!} \mathcal{E}\right)
$$

Il dérive de 1.2.3.2, le diagramme commutatif suivant :

$$
\begin{aligned}
& \mathcal{O}_{S_{i}} \otimes \mathbb{\mathcal { O }}_{\mathcal{S}}^{\mathbb{L}}\left(\mathbb{R} \mathcal{H} m_{\widehat{\mathcal{D}}_{\mathfrak{X}}^{(m)}}\left(f_{+}(\mathcal{F}), \mathcal{E}\right)\right) \stackrel{\delta_{f}}{\sim} \mathcal{O}_{S_{i}} \otimes_{\mathcal{O}_{\mathcal{S}}}^{\mathbb{L}}\left(\mathbb{R} f_{*} \mathbb{R} \mathcal{H} o m_{\widehat{\mathcal{D}}_{\mathcal{Y}}^{(m)}}\left(\mathcal{F}, f^{!} \mathcal{E}\right)\right) \\
& \mathbb{R H o m}_{\mathcal{D}_{X_{i}}^{(m)}}^{\sim \downarrow}\left(f_{i+}\left(\mathcal{F}_{i}\right), \mathcal{E}_{i}\right) \underset{\delta_{f_{i}}}{\sim} \mathbb{R} f_{*} \mathbb{R} \mathcal{H o m}_{\mathcal{D}_{Y_{i}}^{(m)}}^{\sim \downarrow}\left(\mathcal{F}_{i}, f_{i}^{!} \mathcal{E}_{i}\right),
\end{aligned}
$$

où les isomorphisme verticaux se déduisent des isomorphismes de changement de base de 1.2.1 et 1.2.2.

Avec la remarque de 1.2.1.2, on déduit de 1.2.3.4 par application du foncteur $H^{0} \circ \Gamma(\mathfrak{X},-)$, le diagramme commutatif suivant :

$$
\begin{gathered}
\left.\left.\operatorname{Hom}_{\widehat{\mathcal{D}}_{X}^{(m)}}\left(f_{+}(\mathcal{F}), \mathcal{E}\right)\right) \stackrel{\operatorname{adj}_{f}}{\sim} \operatorname{Hom}_{\widehat{\mathcal{D}}_{\mathcal{Y}}^{(m)}}\left(\mathcal{F}, f^{!} \mathcal{E}\right)\right) \\
\downarrow \\
\operatorname{Hom}_{\mathcal{D}_{X_{i}}^{(m)}}\left(f_{i+}\left(\mathcal{F}_{i}\right), \mathcal{E}_{i}\right) \stackrel{\operatorname{adj}_{f_{i}}}{\underset{\sim}{\sim}} \operatorname{Hom}_{\mathcal{D}_{Y_{i}}^{(m)}}\left(\mathcal{F}_{i}, f_{i}^{!} \mathcal{E}_{i}\right),
\end{gathered}
$$

dont les morphismes verticaux sont ceux induits fonctoriellement par $\mathcal{O}_{S_{i}} \otimes_{\mathcal{O}_{\mathcal{S}}}^{\mathbb{L}}$ - .

Si on a seulement $\mathcal{E} \in D_{\mathrm{qc}}^{\mathrm{b}}\left(\widehat{\mathcal{D}}_{\mathfrak{X}}^{(m)}\right)$, on construit l'isomorphisme d'adjonction $\left.\left.\operatorname{adj}_{f}: \operatorname{Hom}_{\widehat{\mathcal{D}}_{\mathfrak{X}}^{(m)}}\left(f_{+}(\mathcal{F}), \mathcal{E}\right)\right) \stackrel{\sim}{\longrightarrow} \operatorname{Hom}_{\widehat{\mathcal{D}}_{\mathcal{Y}}^{(m)}}\left(\mathcal{F}, f^{!} \mathcal{E}\right)\right)$ via la commutativité du diagramme ci-dessous :

$$
\begin{aligned}
& \left.\left.\operatorname{Hom}_{\widehat{\mathcal{D}}_{\mathfrak{X}}^{(m)}}\left(f_{+}(\mathcal{F}), \mathcal{E}\right)\right) \ldots \operatorname{adj}_{f} \ldots \operatorname{Hom}_{\widehat{\mathcal{D}}_{\mathcal{Y}}^{(m)}}\left(\mathcal{F}, f^{!} \mathcal{E}\right)\right)
\end{aligned}
$$

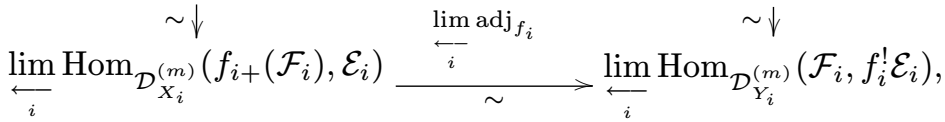

dont les isomorphismes verticaux sont dus à l'équivalence de catégorie ([6, 3.2.3]). Grâce à la commutativité de 1.2.3.5, les deux constructions de l'isomorphisme d'adjonction $\operatorname{adj}_{f}$ sont compatibles.

Pour tous morphismes propres $g: \mathcal{Z} \rightarrow \mathcal{Y}$ et $f: \mathcal{Y} \rightarrow \mathfrak{X}$ de $\mathcal{V}$-schémas formels lisses, pour tout $\mathcal{E} \in D_{\mathrm{qc}}^{\mathrm{b}}\left(\widehat{\mathcal{D}}_{\mathfrak{X}}^{(m)}\right)$ et tout $\mathcal{G} \in D_{\text {coh }}^{\mathrm{b}}\left(\widehat{\mathcal{D}}_{\mathcal{Y}}^{(m)}\right)$, on obtient 
par complétion (voir 1.2.3.6) de 1.1.5.1 le diagramme commutatif suivant

$$
\begin{aligned}
& \operatorname{Hom}_{\widehat{\mathcal{D}}_{\mathfrak{X}}^{(m)}}\left(f_{+} \circ g_{+}(\mathcal{G}), \mathcal{E}\right) \longleftarrow \sim \operatorname{Hom}_{\widehat{\mathcal{D}}_{\mathfrak{X}}^{(m)}}\left((f \circ g)_{+}(\mathcal{G}), \mathcal{E}\right) \\
& \sim \vee \operatorname{adj}_{g} \circ \operatorname{adj}_{f} \quad \sim \downarrow \operatorname{adj}_{f \circ g} \\
& \operatorname{Hom}_{\widehat{\mathcal{D}}_{\mathcal{Z}}^{(m)}}\left(\mathcal{G}, g^{!} f^{!} \mathcal{E}\right) \longleftarrow \operatorname{Hom}_{\widehat{\mathcal{D}}_{\mathcal{Z}}^{(m)}}\left(\mathcal{G},(f \circ g)^{!} \mathcal{E}\right) .
\end{aligned}
$$

1.2.4. - Soient $f: \mathcal{Y} \rightarrow \mathfrak{X}$ un morphisme propre de $\mathcal{V}$-schémas formels lisses, $m^{\prime} \geq m$ deux entiers, $\mathcal{E} \in D_{\mathrm{qc}}^{\mathrm{b}}\left(\widehat{\mathcal{D}}_{\mathfrak{X}}^{(m)}\right), \mathcal{E}^{\prime} \in D_{\mathrm{qc}}^{\mathrm{b}}\left(\widehat{\mathcal{D}}_{\mathfrak{X}}^{\left(m^{\prime}\right)}\right), \mathcal{E} \rightarrow \mathcal{E}^{\prime}$ un morphisme $\widehat{\mathcal{D}}_{\mathfrak{X}}^{(m)}$-linéaire, $\mathcal{F} \in D_{\text {coh }}^{\mathrm{b}}\left(\widehat{\mathcal{D}}_{\mathcal{Y}}^{(m)}\right)$ et $\mathcal{F}^{\prime}:=\widehat{\mathcal{D}}_{\mathcal{Y}}^{\left(m^{\prime}\right)} \otimes \widehat{\mathcal{L}}_{\mathcal{Y}}^{(m)} \mathcal{F}$. On bénéficie, via le diagramme commutatif ci-dessous, du morphisme de changement de niveau, noté niv :

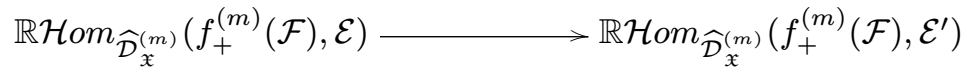

$$
\begin{aligned}
& \forall \text { niv } 1 \sim \\
& \mathbb{R} \mathcal{H o m}_{\widehat{\mathcal{D}}_{\mathfrak{X}}^{\left(m^{\prime}\right)}}\left(f_{+}^{\left(m^{\prime}\right)}\left(\mathcal{F}^{\prime}\right), \mathcal{E}^{\prime}\right) \longrightarrow \mathbb{R} \mathcal{H}{ }_{\widehat{\mathcal{D}}_{\mathfrak{X}}^{\left(m^{\prime}\right)}}\left(\widehat{\mathcal{D}}_{\mathfrak{X}}^{\left(m^{\prime}\right)} \otimes_{\widehat{\mathcal{D}}_{\mathfrak{X}}^{(m)}}^{\mathbb{L}} f_{+}^{(m)}(\mathcal{F}), \mathcal{E}^{\prime}\right),
\end{aligned}
$$

dont l'isomorphisme du bas découle de [6, 3.5.3.(ii)]. On vérifie ensuite la commutativité du diagramme

$$
\begin{aligned}
\mathcal{O}_{S_{i}} \otimes_{\mathcal{O}_{\mathcal{S}}}^{\mathbb{L}}\left(\mathbb{R} \mathcal{H o m}_{\widehat{\mathcal{D}}_{\mathfrak{X}}^{(m)}}\left(f_{+}^{(m)}(\mathcal{F}), \mathcal{E}\right)\right) \stackrel{\text { niv }}{\longrightarrow} \mathcal{O}_{S_{i}} \otimes_{\mathcal{O}_{\mathcal{S}}}^{\mathbb{L}}\left(\mathbb{R} \mathcal{H o m}_{\widehat{\mathcal{D}}_{\mathfrak{X}}^{\left(m^{\prime}\right)}}\left(f_{+}^{\left(m^{\prime}\right)}\left(\mathcal{F}^{\prime}\right), \mathcal{E}^{\prime}\right)\right) \\
\sim \downarrow \\
\sim \downarrow \\
\mathbb{R} \mathcal{H o m}_{\mathcal{D}_{X_{i}}^{(m)}}\left(f_{i+}^{(m)}\left(\mathcal{F}_{i}\right), \mathcal{E}_{i}\right) \longrightarrow \operatorname{niv}_{\mathcal{D}_{X_{i}}^{\left(m^{\prime}\right)}}\left(f_{i+}^{\left(m^{\prime}\right)}\left(\mathcal{F}_{i}^{\prime}\right), \mathcal{E}_{i}^{\prime}\right),
\end{aligned}
$$

dont le morphisme de changement de niveau du bas se construit de manière analogue à 1.2.4.1 et dont les isomorphisme verticaux se déduisent des isomorphismes de changement de base de 1.2 .1 et 1.2.2. Il en résulte celle du suivant :

$$
\begin{gathered}
\operatorname{Hom}_{\widehat{\mathcal{D}}_{\mathfrak{X}}^{(m)}}\left(f_{+}^{(m)}(\mathcal{F}), \mathcal{E}\right) \stackrel{\text { niv }}{\longrightarrow} \operatorname{Hom}_{\widehat{\mathcal{D}}_{\mathfrak{X}}^{\left(m^{\prime}\right)}}\left(f_{+}^{\left(m^{\prime}\right)}\left(\mathcal{F}^{\prime}\right), \mathcal{E}^{\prime}\right) \\
\downarrow \\
\operatorname{Hom}_{\mathcal{D}_{X_{i}}^{(m)}}\left(f_{i+}^{(m)}\left(\mathcal{F}_{i}\right), \mathcal{E}_{i}\right) \stackrel{\text { niv }}{\longrightarrow} \operatorname{Hom}_{\mathcal{D}_{X_{i}}^{\left(m^{\prime}\right)}}\left(f_{i+}^{\left(m^{\prime}\right)}\left(\mathcal{F}_{i}^{\prime}\right), \mathcal{E}_{i}^{\prime}\right) .
\end{gathered}
$$

On a aussi le morphisme de changement de niveau suivant :

$$
\operatorname{niv}: \mathbb{R} f_{*} \mathbb{R} \mathcal{H} o m_{\widehat{\mathcal{D}}_{\mathcal{Y}}^{(m)}}\left(\mathcal{F}, f^{!(m)} \mathcal{E}\right) \rightarrow \mathbb{R} f_{*} \mathbb{R} \mathcal{H} o m_{\widehat{\mathcal{D}}_{\mathcal{Y}}^{(m)}}\left(\mathcal{F}, f^{!\left(m^{\prime}\right)} \mathcal{E}^{\prime}\right) \stackrel{\sim}{\sim} f_{*} \mathbb{R} \mathcal{H} o m_{\widehat{\mathcal{D}}_{\mathcal{Y}}^{\left(m^{\prime}\right)}}\left(\mathcal{F}^{\prime}, f^{!\left(m^{\prime}\right)} \mathcal{E}^{\prime}\right)
$$


De plus, le diagramme

$$
\begin{aligned}
& \mathcal{O}_{S_{i}} \otimes_{\mathcal{O}_{\mathcal{S}}}^{\mathbb{L}}\left(\mathbb{R} f_{*} \mathbb{R} \mathcal{H} o m_{\widehat{\mathcal{D}}_{\mathcal{Y}}^{(m)}}\left(\mathcal{F}, f^{!(m)} \mathcal{E}\right)\right) \stackrel{\text { niv }}{\longrightarrow} \mathcal{O}_{S_{i}} \otimes_{\mathcal{O}_{\mathcal{S}}}^{\mathbb{L}}\left(\mathbb{R} f_{*} \mathbb{R} \mathcal{H} o m_{\widehat{\mathcal{D}}_{\mathcal{Y}}^{\left(m^{\prime}\right)}}\left(\mathcal{F}^{\prime}, f^{!^{\left(m^{\prime}\right)}} \mathcal{E}^{\prime}\right)\right) \\
& \sim \downarrow \quad \sim \downarrow \\
& \mathbb{R} f_{*} \mathbb{R} \mathcal{H o m}{ }_{\mathcal{D}_{Y_{i}}^{(m)}}\left(\mathcal{F}_{i}, f_{i}^{!(m)} \mathcal{E}_{i}\right) \quad \text { niv } \longrightarrow \mathbb{R} f_{*} \mathbb{R} \mathcal{H} o m_{\mathcal{D}_{Y_{i}^{\prime}}^{\left(m^{\prime}\right)}}\left(\mathcal{F}_{i}^{\prime}, f_{i}^{!^{\left(m^{\prime}\right)}} \mathcal{E}_{i}^{\prime}\right),
\end{aligned}
$$

dont le morphisme du bas se définit comme dans 1.2.4.4 et dont les isomorphisme verticaux se déduisent des isomorphismes de changement de base de 1.2.1 et 1.2.2, est commutatif. On obtient ainsi la commutativité du suivant :

$$
\begin{gathered}
\operatorname{Hom}_{\widehat{\mathcal{D}}_{\mathcal{Y}}^{(m)}}\left(\mathcal{F}, f^{! !(m)} \mathcal{E}\right) \stackrel{\text { niv }}{\longrightarrow} \\
\operatorname{Hom}_{\widehat{\mathcal{D}}_{\mathcal{Y}}^{\left(m^{\prime}\right)}}\left(\mathcal{F}^{\prime}, f^{!\left(m^{\prime}\right)} \mathcal{E}^{\prime}\right) \\
\downarrow \\
\operatorname{Hom}_{\mathcal{D}_{Y_{i}}^{(m)}}\left(\mathcal{F}_{i}, f_{i}^{!(m)} \mathcal{E}_{i}\right) \stackrel{\text { niv }}{\longrightarrow} \operatorname{Hom}_{\mathcal{D}_{Y_{i}^{\prime}}^{\left(m^{\prime}\right)}}\left(\mathcal{F}_{i}^{\prime}, f_{i}^{!\left(m^{\prime}\right)} \mathcal{E}_{i}^{\prime}\right) .
\end{gathered}
$$

Considérons enfin le diagramme

$(1.2 .4 .7)$

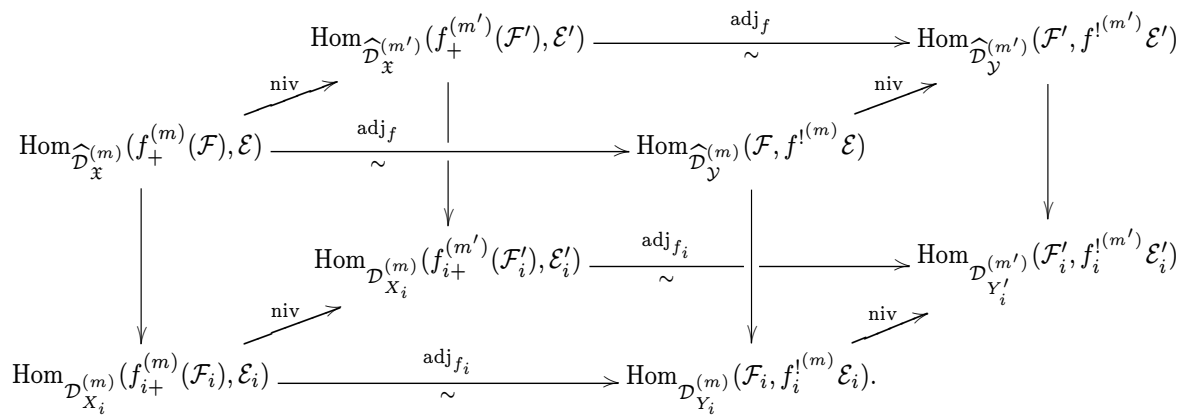

Par 1.2.4.3 (resp. 1.2.4.6, resp. 1.2.3.5), le carré de gauche (resp. de droite, resp. de devant et de derrière) sont commutatifs. La commutativité de l'isomorphisme de dualité relative de $f_{i}$ au changement de niveau implique celle de $\delta_{f_{i}}$ puis celle de $\operatorname{adj}_{f_{i}}$. Le carré du bas est ainsi commutatif. Or, en passant à la limite projective sur $i$, les flèches verticales de 1.2.4.7 deviennent des isomorphismes. Il en résulte la commutativité du carré supérieur de 1.2.4.7.

1.2.5. - Soit $f: \mathcal{Y} \rightarrow \mathfrak{X}$ un morphisme propre de $\mathcal{V}$-schémas formels lisses. Pour tous $\mathcal{E}^{(\bullet)} \in L D_{\mathbb{Q}, \mathrm{qc}}^{\mathrm{b}}\left(\widehat{\mathcal{D}}_{\mathfrak{X}}^{(\bullet)}\right), \mathcal{F}^{(\bullet)} \in L D_{\mathbb{Q}, \text { coh }}^{\mathrm{b}}\left(\widehat{\mathcal{D}}_{\mathcal{Y}}^{(\bullet)}\right)$, par construction des catégories de la forme $L D_{\mathbb{Q}, \mathrm{qc}}^{\mathrm{b}}\left(\widehat{\mathcal{D}}_{\mathfrak{X}}^{(\bullet)}\right)$, de la commutativité au changement de niveau des isomorphismes d'adjonction de 1.2.3.6 (i.e. commutativité du carré 
supérieur de 1.2.4.7), l'isomorphisme canonique d'adjonction suivant :

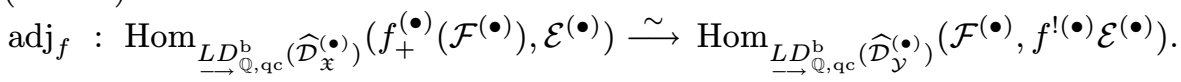

Pour tout morphisme propre $g: \mathcal{Z} \rightarrow \mathcal{Y}$ de $\mathcal{V}$-schémas formels lisses, pour tout $\mathcal{E}^{(\bullet)} \in \underset{\sim}{L} D_{\mathbb{Q}, \mathrm{qc}}^{\mathrm{b}}\left(\widehat{\mathcal{D}}_{\mathfrak{X}}^{(\bullet)}\right)$ et tout $\mathcal{G}^{(\bullet)} \in L{ }_{\rightarrow} D_{\mathbb{Q}, \text { coh }}^{\mathrm{b}}\left(\widehat{\mathcal{D}}_{\mathcal{Z}}^{(\bullet)}\right)$, il dérive de 1.2.3.7 le diagramme commutatif :

$$
\begin{aligned}
& \operatorname{Hom}_{\underline{L D_{\mathbb{Q}, \mathrm{qc}}^{\mathrm{b}}}}\left(\widehat{\mathcal{D}}_{\mathfrak{X}}^{(\bullet \bullet)}\right)\left(f_{+} \circ g_{+}\left(\mathcal{G}^{(\bullet)}\right), \mathcal{E}^{(\bullet)}\right) \longleftarrow \sim \operatorname{Hom}_{\underline{L} D_{\mathbb{Q}, \mathrm{qc}}^{\mathrm{b}}\left(\widehat{\mathcal{D}}_{\mathfrak{X}}^{(\bullet)}\right)}\left((f \circ g)_{+}\left(\mathcal{G}^{(\bullet)}\right), \mathcal{E}^{(\bullet)}\right) \\
& \sim \vee \operatorname{adj}_{g} \circ \operatorname{adj}_{f} \\
& \sim \vee \operatorname{adj}_{f \circ g}
\end{aligned}
$$

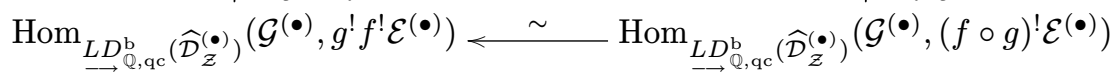

1.2.6. - Soient $f: \mathcal{Y} \rightarrow \mathfrak{X}$ un morphisme propre de $\mathcal{V}$-schémas formels lisses, $\mathcal{E}^{(\bullet)} \in L D_{\mathbb{Q}, \text { coh }}^{\mathrm{b}}\left(\widehat{\mathcal{D}}_{\mathfrak{X}}^{(\bullet)}\right), \mathcal{F}^{(\bullet)} \in L D_{\mathbb{Q}, \text { coh }}^{\mathrm{b}}\left(\widehat{\mathcal{D}}_{\mathcal{Y}}^{(\bullet)}\right), \mathcal{E}:=\lim ^{(\bullet)}$ et $\mathcal{F}:=\lim \mathcal{F}^{(\bullet)}(\mathrm{l}$ foncteur lim est celui défini dans $[6,4.2 .2])$. On bénéficie à nouveau de l'isomor-

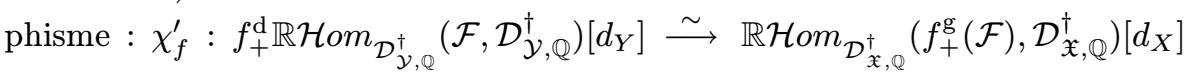
(voir [32]). On construit ensuite de manière analogue à 1.1.3.3, l'isomorphisme $\delta_{f}: \mathbb{R H}_{\mathcal{H}^{\prime} m_{\mathcal{Q}}^{\dagger}}\left(f_{+}(\mathcal{F}), \mathcal{E}\right) \stackrel{\sim}{\longrightarrow} \mathbb{R} f_{*} \mathbb{R} \mathcal{H}$ om $_{\mathcal{D}_{\mathcal{Y}, \mathbb{Q}}^{\dagger}}\left(\mathcal{F}, f^{!} \mathcal{E}\right)$, puis l'isomorphisme d'adjonction : $\operatorname{adj}_{f}: \operatorname{Hom}_{\mathcal{D}_{\mathfrak{X}, \mathbb{Q}}^{\dagger}}\left(f_{+}(\mathcal{F}), \mathcal{E}\right) \stackrel{\sim}{\longrightarrow} \operatorname{Hom}_{\mathcal{D}_{\mathcal{Y}, \mathbb{Q}}^{\dagger}}\left(\mathcal{F}, f^{!} \mathcal{E}\right)$.

Le diagramme suivant

$$
\begin{aligned}
& \operatorname{Hom}_{\underset{L D_{\mathbb{Q}, \mathrm{qc}}^{\mathrm{b}}}{\mathrm{b}}\left(\widehat{\mathcal{D}}_{\mathfrak{X}}^{(\bullet)}\right)}\left(f_{+}^{(\bullet)}\left(\mathcal{F}^{(\bullet)}\right), \mathcal{E}^{(\bullet)}\right) \stackrel{\text { adj }}{\sim} \operatorname{Hom}_{\underset{\sim}{\rightarrow D_{\mathbb{Q}, \mathrm{qc}}^{\mathrm{b}}}}\left(\widehat{\mathcal{D}}_{\mathcal{Y}}^{(\bullet)}\right)\left(\mathcal{F}^{(\bullet)}, f^{!(\bullet)} \mathcal{E}^{(\bullet)}\right) \\
& \sim \lim _{\rightarrow \rightarrow} \quad \lim _{\rightarrow}^{\rightarrow} \\
& \operatorname{Hom}_{\mathcal{D}_{\mathfrak{X}, \mathbb{Q}}^{\dagger}}\left(f_{+}(\mathcal{F}), \mathcal{E}\right) \underset{\sim}{\sim} \operatorname{Hom}_{\mathcal{D}_{\mathcal{Y}, \mathbb{Q}}^{\dagger}}\left(\mathcal{F}, f^{!} \mathcal{E}\right),
\end{aligned}
$$

où l'isomorphisme du haut est 1.2.5.1, est commutatif.

En effet, quitte à compliquer les notations, supposons que $\mathcal{E}^{(m)} \in$ $D_{\text {coh }}^{\mathrm{b}}\left(\widehat{\mathcal{D}}_{\mathfrak{X}}^{(m)}\right)$, le morphisme $\widehat{\mathcal{D}}_{\mathfrak{X}}^{\left(m^{\prime}\right)} \otimes \widehat{\mathcal{D}}_{\mathfrak{X}}^{(m)} \mathcal{E}^{(m)} \rightarrow \mathcal{E}^{\left(m^{\prime}\right)}$ est un isomorphisme et de même en remplaçant $\mathcal{E}$ par $\mathcal{F}$. On dispose du diagramme commutatif : $(1.2 .6 .2)$

$$
\begin{aligned}
& f_{+}^{(m) \mathrm{d}} \mathbb{R} \mathcal{H} m_{\mathcal{D}_{\mathcal{Y}}^{(m)}}\left(\mathcal{F}^{(m)}, \mathcal{D}_{\mathcal{Y}}^{(m)}\right)\left[d_{Y}\right] \otimes_{\mathcal{D}_{\mathfrak{X}}^{(m)}} \mathcal{D}_{\mathfrak{X}, \mathbb{Q}}^{\dagger} \stackrel{\chi_{f}^{\prime}}{\sim} \mathbb{R} \mathcal{H} o m_{\mathcal{D}_{\mathfrak{X}}^{(m)}}\left(f_{+}^{(m) g}\left(\mathcal{F}^{(m)}\right), \mathcal{D}_{\mathfrak{X}}^{(m)}\right)\left[d_{X}\right] \otimes_{\mathcal{D}_{\mathfrak{X}}^{(m)}} \mathcal{D}_{\mathfrak{X}, \mathbb{Q}}^{\dagger}
\end{aligned}
$$

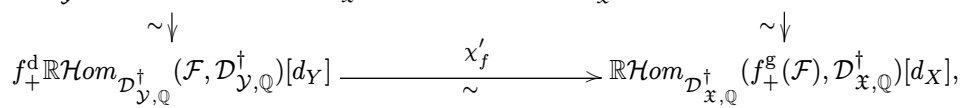

dont les isomorphismes verticaux se déduisent de [6, 3.5.3.(ii)] et de la commutation des foncteurs duaux à l'extension des scalaires. Il en dérive le carré 
commutatif suivant :

$$
\begin{gathered}
\operatorname{Hom}_{\mathcal{D}_{\mathfrak{X}}^{(m)}}\left(f_{+}^{(m)}\left(\mathcal{F}^{(m)}\right), \mathcal{E}^{(m)}\right) \stackrel{\operatorname{adj}_{f}}{\sim} \operatorname{Hom}_{\mathcal{D}_{\mathcal{Y}}^{(m)}}\left(\mathcal{F}^{(m)}, f^{!(m)} \mathcal{E}^{(m)}\right) \\
\sim \downarrow \\
\operatorname{Hom}_{\mathcal{D}_{\mathfrak{X}, \mathbb{Q}}^{\dagger}}\left(f_{+}(\mathcal{F}), \mathcal{E}\right) \underset{\sim}{\sim} \frac{\operatorname{adj}_{f}}{\sim} \operatorname{Hom}_{\mathcal{D}_{\mathcal{Y}, \mathbb{Q}}^{\dagger}}\left(\mathcal{F}, f^{!} \mathcal{E}\right) .
\end{gathered}
$$

Avec les notations de [6, 4.2.2], il en découle, pour tous $\lambda \in L, \chi \in M$, le diagramme commutatif suivant :

$(1.2 .6 .4)$

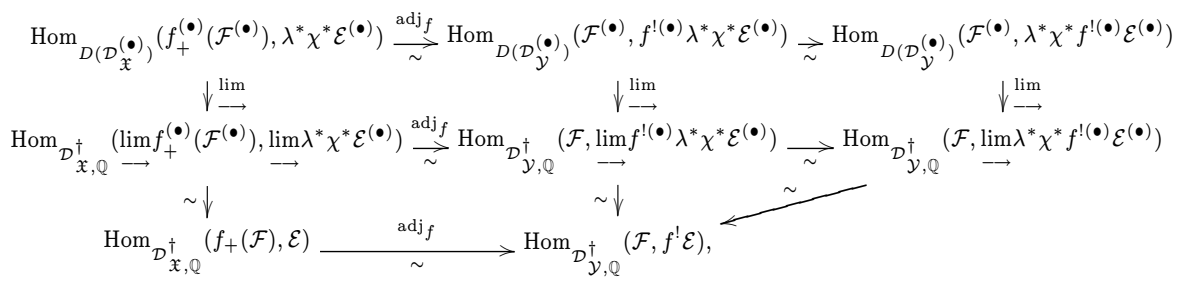

où lim est le foncteur de [6, 4.2.2] (ou [6, 4.2.4]).

En passant à la limite inductive sur $\lambda \in L, \chi \in M$, on déduit de 1.2.6.4 le diagramme 1.2.6.1.

1.2.7. - Soient $f: \mathcal{Y} \rightarrow \mathfrak{X}$ un morphisme propre de $\mathcal{V}$-schémas formels lisses, $T$ un diviseur de $X$ tel que $T^{\prime}:=f^{-1}(T)$ soit un diviseur de $Y$.

D'après $[12,1.2 .10]$, pour tous $\mathcal{E} \in D_{\text {coh }}^{\mathrm{b}}\left({ }^{\mathrm{g}} \mathcal{D}_{\mathfrak{X}}^{\dagger}\left({ }^{\dagger} T\right)_{\mathbb{Q}}\right)$ et $\mathcal{F} \in D_{\text {coh }}^{\mathrm{b}}\left(\mathcal{D}_{\mathcal{Y}}^{\dagger}\left({ }^{\dagger} T^{\prime}\right)_{\mathbb{Q}}{ }^{\mathrm{d}}\right)$, on dispose de l'isomorphisme canonique d'adjonction :

$$
\operatorname{adj}_{f, T}: \operatorname{Hom}_{\left.\mathcal{D}_{\mathcal{X}}^{\dagger}{ }^{\dagger} T\right)_{\mathbb{Q}}}\left(f_{T+}(\mathcal{F}), \mathcal{E}\right) \stackrel{\sim}{\longrightarrow} \operatorname{Hom}_{\mathcal{D}_{\mathcal{Y}}^{\dagger}\left({ }^{\dagger} T^{\prime}\right)_{\mathbb{Q}}}\left(\mathcal{F}, f_{T}^{!} \mathcal{E}\right) .
$$

Il en découle comme d'habitude le morphisme d'adjonction : $\operatorname{adj}_{f, T, \mathcal{F}}:$ $\mathcal{F} \rightarrow f_{T}^{!} f_{T+}(\mathcal{F})$. De même, si $f_{T}^{!} \mathcal{E} \in D_{\text {coh }}^{\mathrm{b}}\left(\mathcal{D}_{\mathcal{Y}}^{\dagger}\left({ }^{\dagger} T^{\prime}\right)_{\mathbb{Q}}{ }^{\mathrm{d}}\right)$, il en dérive le morphisme d'adjonction : $\operatorname{adj}_{f, T, \mathcal{E}}: f_{T+} f_{T}^{!}(\mathcal{E}) \rightarrow \mathcal{E}$. Lorsqu'aucune confusion n'est à craindre, on les notera simplement $\operatorname{adj}_{f, T}, \operatorname{adj}_{f}$ etc. Lorsque le diviseur est vide, on ne l'indiquera pas. Le morphisme d'adjonction $\operatorname{adj}_{f}$ de 1.2 .6 est le même que 1.2.7.1.

Proposition 1.2.8. - Soient $g: \mathcal{Z} \rightarrow \mathcal{Y}$ et $f: \mathcal{Y} \rightarrow \mathfrak{X}$ deux morphismes propres de $\mathcal{V}$-schémas formels lisses, $T$ un diviseur de $X$ tel que $T^{\prime}:=f^{-1}(T)$ (resp. $T^{\prime \prime}:=g^{-1}\left(T^{\prime}\right)$ ) soit un diviseur de $Y$ (resp. $Z$ ).

Pour tout $\mathcal{G} \in D_{\mathrm{coh}}^{\mathrm{b}}\left({ }^{\mathrm{g}} \mathcal{D}_{\mathcal{Z}}^{\dagger}\left(T^{\prime \prime}\right)_{\mathbb{Q}}\right)$ et pour tout $\mathcal{E} \in D_{\mathrm{coh}}^{\mathrm{b}}\left({ }^{\mathrm{g}} \mathcal{D}_{\mathfrak{X}}^{\dagger}(T)_{\mathbb{Q}}\right)$ tel que $f_{T}^{!}(\mathcal{E}) \in D_{\text {coh }}^{\mathrm{b}}\left({ }^{\mathrm{g}} \mathcal{D}_{\mathcal{Y}}^{\dagger}\left(T^{\prime}\right)_{\mathbb{Q}}\right)$ et $(f \circ g)_{T}^{!}(\mathcal{E}) \in D_{\text {coh }}^{\mathrm{b}}\left({ }^{\mathrm{g}} \mathcal{D}_{\mathcal{Z}}^{\dagger}\left(T^{\prime \prime}\right)_{\mathbb{Q}}\right)$, le diagramme

$$
\begin{aligned}
\operatorname{Hom}_{\mathcal{D}_{\mathfrak{X}}^{\dagger}(T)_{\mathbb{Q}}}( & \left.f_{T+} \circ g_{T^{\prime}+}(\mathcal{G}), \mathcal{E}\right) \longleftarrow \sim \operatorname{Hom}_{\mathcal{D}_{\mathcal{X}}^{\dagger}(T)_{\mathbb{Q}}}\left((f \circ g)_{+}(\mathcal{G}), \mathcal{E}\right) \\
\sim \downarrow \operatorname{adj}_{g, T^{\prime}} \circ \operatorname{adj}_{f, T} & \sim \operatorname{adj}_{f \circ g, T} \\
\operatorname{Hom}_{\mathcal{D}_{\mathcal{Z}}^{\dagger}\left(T^{\prime \prime}\right)_{\mathbb{Q}}}\left(\mathcal{G}, g^{!} f^{!} \mathcal{E}\right) \longleftarrow & \sim \sim \operatorname{Hom}_{\mathcal{D}_{\mathcal{Z}}^{\dagger}\left(T^{\prime \prime}\right)_{\mathbb{Q}}}\left(\mathcal{G},(f \circ g)^{!} \mathcal{E}\right),
\end{aligned}
$$


où les isomorphismes horizontaux sont induits par les isomorphismes canoniques de composition (1.1.2), est commutatif.

Démonstration. - Par [6, 4.3.12], on peut supposer le diviseur $T$ vide. Cela découle alors de 1.2.5.2 et 1.2.6.1.

REMARQues 1.2.9. - Avec les notations et hypothèses de 1.2.8, l'hypothèse « $f_{T}^{!}(\mathcal{E}) \in D_{\text {coh }}^{\mathrm{b}}\left({ }^{\mathrm{g}} \mathcal{D}_{\mathcal{Y}}^{\dagger}\left(T^{\prime}\right)_{\mathbb{Q}}\right)$ et $(f \circ g)_{T}^{!}(\mathcal{E}) \in D_{\text {coh }}^{\mathrm{b}}\left({ }^{\mathrm{g}} \mathcal{D}_{\mathcal{Z}}^{\dagger}\left(T^{\prime \prime}\right)_{\mathbb{Q}}\right) »$ est toujours satisfaite lorsque $f$ et $g$ sont, en plus d'être propres, lisses. De plus, ces hypothèses sont aussi validées lorsque l'on travaille avec des complexes surcohérents (voir [9]) etc. Dans ces conditions, il découle de 1.2.8 que l'isomorphisme canonique de composition des images directes utilisé ici (voir 1.1.2.1) est le même que celui construit dans $[12,1.2 .15]$. On a ainsi obtenu une unification.

LEMme 1.2.10. - Soient $f: \mathcal{Y} \rightarrow \mathfrak{X}$ un morphisme propre de $\mathcal{V}$-schémas formels séparés lisses, $T$ un diviseur de $X$ tel que $T^{\prime}:=f^{-1}(T)$ soit un diviseur de $Y$, et $\mathcal{F} \in D_{\text {coh }}^{\mathrm{b}}\left({ }^{\mathrm{g}} \mathcal{D}_{\mathcal{Y}}^{\dagger}\left(T^{\prime}\right)_{\mathbb{Q}}\right)$.

Alors, $f_{T}^{!} \circ f_{T,+}(\mathcal{F}) \in D_{\text {coh }}^{\mathrm{b}}\left({ }^{\mathrm{g}} \mathcal{D}_{\mathcal{Y}}^{\dagger}\left(T^{\prime}\right)_{\mathbb{Q}}\right)$.

Démonstration. - Quitte à compliquer les notations, supposons le diviseur $T$ vide. On note $\delta_{f}=(\mathrm{id}, f): \mathcal{Y} \hookrightarrow \mathcal{Y} \times \mathfrak{X}, \mathcal{E}:=\delta_{f,+}(\mathcal{F}), p: \mathcal{Y} \times \mathfrak{X} \rightarrow \mathfrak{X}$ la deuxième projection, $g=f \times$ id $: \mathcal{Y} \times \mathfrak{X} \rightarrow \mathfrak{X} \times \mathfrak{X}$ et $\delta: \mathfrak{X} \hookrightarrow \mathfrak{X} \times \mathfrak{X}$ l'immersion fermée diagonale. D'après [19, 5.3.7], le carré suivant

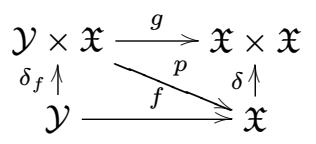

est cartésien. Comme $g_{+}(\mathcal{E})=g_{+} \circ \delta_{f,+}(\mathcal{F}) \stackrel{\sim}{\longrightarrow} \delta_{+} \circ f_{+}(\mathcal{F}), g_{+}(\mathcal{E})$ est à support dans $X$ (via $\delta)$. Comme le carré de 1.2.10.1 est cartésien, $g^{!} \circ g_{+}(\mathcal{E})$ est à support dans $Y\left(\operatorname{via} \delta_{f}\right)$. Or, $p^{!} \circ p_{+}(\mathcal{E}) \stackrel{\sim}{\longrightarrow} p^{!} \circ \delta^{!} \circ \delta_{+} \circ p_{+}(\mathcal{E}) \stackrel{\sim}{\longrightarrow} g^{!} \circ g_{+}(\mathcal{E})$. Ainsi, $p^{!} \circ p_{+}(\mathcal{E})$ est à support dans $Y$. Or, $p_{+}(\mathcal{E}) \stackrel{\sim}{\longrightarrow} f_{+}(\mathcal{F}) \in D_{\text {coh }}^{\mathrm{b}}\left({ }^{\mathrm{g}} \mathcal{D}_{\mathfrak{X}, \mathbb{Q}}^{\dagger}\right)($ car comme $f$ est propre, la cohérence est préservée par image directe). Comme $p$ est lisse, il en découle que $p^{!} \circ p_{+}(\mathcal{E}) \in D_{\text {coh }}^{\mathrm{b}}\left({ }^{\mathrm{g}} \mathcal{D}_{\mathcal{Y} \times \mathfrak{X}, \mathbb{Q}}^{\dagger}\right)$. D'après l'analogue $p$-adique de Berthelot du théorème de Kashiwara, il en résulte que $\delta_{f}^{!}\left(p^{!} \circ\right.$ $\left.p_{+}(\mathcal{E})\right) \in D_{\text {coh }}^{\mathrm{b}}\left({ }^{\mathrm{g}} \mathcal{D}_{\mathcal{Y}, \mathbb{Q}}^{\dagger}\right)$. On conclut en remarquant $: \delta_{f}^{!}\left(p^{!} \circ p_{+}(\mathcal{E})\right)=\delta_{f}^{!} \circ p^{!} \circ$ $p_{+} \circ \delta_{f,+}(\mathcal{F}) \stackrel{\sim}{\longrightarrow} f^{!} \circ f_{+}(\mathcal{F})$.

Proposition 1.2.11. - Soient $g: \mathcal{Z} \rightarrow \mathcal{Y}$ et $f: \mathcal{Y} \rightarrow \mathfrak{X}$ deux morphismes propres de $\mathcal{V}$-schémas formels séparés et lisses, $T$ un diviseur de $X$ tel que $T^{\prime}:=f^{-1}(T)$ (resp. $\left.T^{\prime \prime}:=g^{-1}\left(T^{\prime}\right)\right)$ soit un diviseur de $Y$ (resp. Z). 
Pour tout $\mathcal{G} \in D_{\text {coh }}^{\mathrm{b}}\left({ }^{\mathrm{g}} \mathcal{D}_{\mathcal{Z}}^{\dagger}\left(T^{\prime \prime}\right)_{\mathbb{Q}}\right)$ et pour tout $\mathcal{E} \in D_{\text {coh }}^{\mathrm{b}}\left({ }^{\mathrm{g}} \mathcal{D}_{\mathfrak{X}}^{\dagger}(T)_{\mathbb{Q}}\right)$ tel que $f_{T}^{!}(\mathcal{E}) \in D_{\mathrm{coh}}^{\mathrm{b}}\left({ }^{\mathrm{g}} \mathcal{D}_{\mathcal{Y}}^{\dagger}\left(T^{\prime}\right)_{\mathbb{Q}}\right)$ et $(f \circ g)_{T}^{!}(\mathcal{E}) \in D_{\mathrm{coh}}^{\mathrm{b}}\left({ }^{\mathrm{g}} \mathcal{D}_{\mathcal{Z}}^{\dagger}\left(T^{\prime \prime}\right)_{\mathbb{Q}}\right)$, on dispose des diagrammes commutatifs :

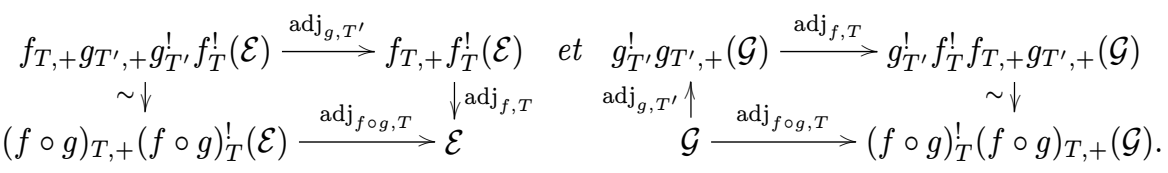

Démonstration. - Par [4, 4.3.12] et via 1.2.10 (pour le diagramme de droite), on se ramène au cas où le diviseur $T$ est vide. En reprenant la preuve de 1.1.12 (qui utilise 1.1.10), cela dérive alors de 1.2.8.

Remarques 1.2.12. - L'hypothèse que $X$ soit séparé est inutile pour valider la commutativité du diagramme de gauche de 1.2.11.1 car celle-ci n'utilise pas le lemme 1.2.10. Il en est de même pour le diagramme de droite lorsque $f$ est une immersion fermée ou lorsque les termes de ce diagramme sont $\mathcal{D}_{\mathcal{Z}}^{\dagger}\left(T^{\prime \prime}\right) \mathbb{Q}^{-c o h e ́ r e n t s . ~}$

\section{Foncteur $\mathrm{sp}_{+}$associant un $\mathcal{D}$-module arithmétique à un isocristal surconvergent. Cas lisse}

Nous adopterons les notations suivantes : si $f: \mathcal{P}^{\prime} \rightarrow \mathcal{P}$ est un morphisme de $\mathcal{V}$-schémas formels lisses, on notera $f_{K}: \mathcal{P}^{\prime}{ }_{K} \rightarrow \mathcal{P}_{K}$ le morphisme d'espaces analytiques rigides associé à $f$, tandis que sp $: \mathcal{P}_{K} \rightarrow \mathcal{P}\left(\right.$ ou sp : $\left.\mathcal{P}_{K}^{\prime} \rightarrow \mathcal{P}^{\prime}\right)$ sera le morphisme de spécialisation (voir [3] ou [28]).

Si $j: Y \hookrightarrow X$ est une immersion ouverte de $k$-schémas de type fini, telle qu'il existe une immersion fermée $X \hookrightarrow \mathcal{P}$, avec $\mathcal{P}$ un $\mathcal{V}$-schéma formel lisse sur un voisinage de $X$, alors $j^{\dagger}$ signifiera le foncteur faisceau des germes de sections surconvergentes le long de $X \backslash Y([3,2.1 .1])$.

\subsection{Isomorphismes de recollement : cas formel}

2.1.1. - Considérons le diagramme commutatif de morphismes de $\mathbb{Z}_{(p)^{-}}$ schémas

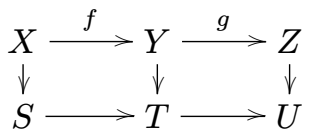

dans lequel $X, Y$ et $Z$ sont respectivement lisses sur $S, T$ et $U$. De plus, on suppose que les schémas $S, T$ et $U$ sont munis respectivement pour un entier $m$ donné de $m$-PD-idéaux quasi-cohérents $m$-PD-nilpotents $\left(\mathfrak{a}_{S}, \mathfrak{b}_{S}, \alpha_{S}\right) \subset \mathcal{O}_{S}$, $\left(\mathfrak{a}_{T}, \mathfrak{b}_{T}, \alpha_{T}\right) \subset \mathcal{O}_{T}$ et $\left(\mathfrak{a}_{U}, \mathfrak{b}_{U}, \alpha_{U}\right) \subset \mathcal{O}_{U}$ de telle manière que les morphismes 
$S \rightarrow T$ et $T \rightarrow U$ soient des $m$-PD-morphismes. On note $X_{0}, Y_{0}$ et $Z_{0}$ les réductions respectives de $X, Y$ et $Z$ modulo $\mathfrak{a}_{S}, \mathfrak{a}_{T}$ et $\mathfrak{a}_{U}$. On désigne ensuite par $f_{0}$ : $X_{0} \rightarrow Y_{0}$ et $g_{0}: Y_{0} \rightarrow Z_{0}$ les morphismes induits par $f$ et $g$. Soient respectivement $H_{X}, H_{Y}$ et $H_{Z}$ des diviseurs de $X_{0}, Y_{0}$ et $Z_{0}$ induisant les factorisations $f_{0}^{-1}\left(H_{Y}\right) \hookrightarrow H_{X}$ et $g_{0}^{-1}\left(H_{Z}\right) \hookrightarrow H_{Y}$. En désignant par $n_{m} \geq m$ un entier, avec les notations de [21, 2.1], on dispose alors de morphismes $f^{*} \mathcal{B}_{Y}^{\left(n_{m}\right)}\left(H_{Y}\right) \rightarrow$ $\mathcal{B}_{X}^{\left(n_{m}\right)}\left(H_{X}\right)$ et $g^{*} \mathcal{B}_{Z}^{\left(n_{m}\right)}\left(H_{Z}\right) \rightarrow \mathcal{B}_{Y}^{\left(n_{m}\right)}\left(H_{Y}\right)([21,2.1 .2])$. On en déduit le foncteur $f_{H_{Y}, H_{X}+}: D^{-}\left(\mathcal{B}_{X}^{\left(n_{m}\right)}\left(H_{X}\right) \otimes_{\mathcal{O}_{X}} \mathcal{D}_{X / S}^{(m)}\right) \rightarrow D^{-}\left(\mathcal{B}_{Y}^{\left(n_{m}\right)}\left(H_{Y}\right) \otimes_{\mathcal{O}_{Y}} \mathcal{D}_{Y / T}^{(m)}\right)$ « image directe par $f$ à singularités surconvergentes le long de $H_{X}$ et $H_{Y}$ » et le foncteur $f_{H_{X}, H_{Y}}^{!}: D^{-}\left(\mathcal{B}_{Y}^{\left(n_{m}\right)}\left(H_{Y}\right) \otimes \mathcal{O}_{Y} \mathcal{D}_{Y / T}^{(m)}\right) \rightarrow D^{-}\left(\mathcal{B}_{X}^{\left(n_{m}\right)}\left(H_{X}\right) \otimes_{\mathcal{O}_{X}} \mathcal{D}_{X / S}^{(m)}\right)$ « image inverse extraordinaires par $f$ à singularités surconvergentes le long de $H_{X}$ et $H_{Y} \gg$ (e.g., voir [12, 1.1.6,1.1.7]).

En notant $\widetilde{X}, \widetilde{Y}$ et $\widetilde{Z}$ les espaces annelés $\left(X, \mathcal{B}_{X}^{\left(n_{m}\right)}\left(H_{X}\right)\right),\left(Y, \mathcal{B}_{Y}^{\left(n_{m}\right)}\left(H_{Y}\right)\right)$ et $\left(Z, \mathcal{B}_{Z}^{\left(n_{m}\right)}\left(H_{Z}\right)\right)$, on obtient des morphismes d'espaces annelés $\tilde{f}: \widetilde{X} \rightarrow \widetilde{Y}$ et $\tilde{g}: \widetilde{Y} \rightarrow \widetilde{Z}$. Leur réduction modulo a donne des morphismes que l'on notera $\tilde{f}_{0}: \widetilde{X}_{0} \rightarrow \widetilde{Y}_{0}$ et $\tilde{g}_{0}: \widetilde{Y}_{0} \rightarrow \widetilde{Z}_{0}$.

On pose $\widetilde{\mathcal{P}}_{X / S(m)}^{n}:=\mathcal{B}_{X}^{\left(n_{m}\right)}\left(H_{X}\right) \otimes_{\mathcal{O}_{X}} \mathcal{P}_{X / S(m)}^{n}, \widetilde{\mathcal{P}}_{Y / T(m)}^{n}:=\mathcal{B}_{Y}^{\left(n_{m}\right)}\left(H_{Y}\right) \otimes_{\mathcal{O}_{Y}}$ $\mathcal{P}_{Y / T(m)}^{n}$ et $\widetilde{\mathcal{P}}_{Z / U(m)}^{n}:=\mathcal{B}_{Z}^{\left(n_{m}\right)}\left(H_{Z}\right) \otimes_{\mathcal{O}_{Z}} \mathcal{P}_{Z / U(m)}^{n}$.

Le faisceau $\widetilde{\mathcal{P}}_{X / S(m)}^{n}$ est muni de deux structures canoniques (à droite et à gauche) de $\mathcal{B}_{X}^{\left(n_{m}\right)}\left(H_{X}\right)$-algèbres (de même pour $\widetilde{\mathcal{P}}_{Y / T(m)}^{n}$ et $\left.\widetilde{\mathcal{P}}_{Z / U(m)}^{n}\right)$. Lorsqu'aucune confusion n'est à craindre, on omet d'indiquer la base $(S, T$ ou $U)$. On notera $\widetilde{\mathcal{D}}_{X / S}^{(m)}=\mathcal{B}_{X}^{\left(n_{m}\right)}\left(H_{X}\right) \otimes_{\mathcal{O}_{X}} \mathcal{D}_{X / S}^{(m)}, \widetilde{\mathcal{D}}_{Y / T}^{(m)}=\mathcal{B}_{Y}^{\left(n_{m}\right)}\left(H_{Y}\right) \otimes_{\mathcal{O}_{Y}} \mathcal{D}_{Y / T}^{(m)}$ et $\tilde{f}^{!}: D^{-}\left(\widetilde{\mathcal{D}}_{Y / T}^{(m)}\right) \rightarrow D^{-}\left(\widetilde{\mathcal{D}}_{X / S}^{(m)}\right)$, le foncteur image inverse extraordinaire.

D'après [4, 2.1.5] et avec les notations de [4, 2.1.2], pour tout $S$-morphisme $f^{\prime}: X \rightarrow Y$ ayant la même restriction $X_{0} \rightarrow Y_{0}$ que $f$, on dispose, pour $n$ assez grand, de la factorisation $\delta_{f, f^{\prime}}^{n}: X \rightarrow \Delta_{Y / T(m)}^{n}$ (grâce à la $m$-PD-nilpotence de $\left.\left(\mathfrak{a}_{S}, \mathfrak{b}_{S}, \alpha_{S}\right)\right)$ rendant commutatif le diagramme

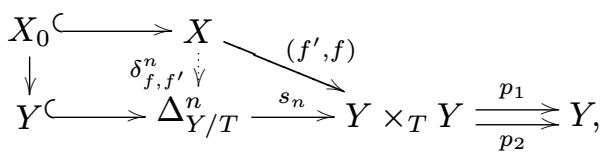

où $p_{1}$ et $p_{2}$ désignent respectivement les projections à gauche et à droite et $s_{n}$ est le morphisme canonique. La structure de $\widetilde{\mathcal{D}}_{Y / T}^{(m)}$-module de $\mathcal{B}_{Y}^{\left(n_{m}\right)}\left(H_{Y}\right)$ compatible à sa structure d'algèbre donne l'isomorphisme de $\mathcal{O}_{\Delta_{Y / T}^{n}}$-algèbres $\left(p_{2} \circ s_{n}\right)^{*}\left(\mathcal{B}_{Y}^{\left(n_{m}\right)}\left(H_{Y}\right)\right) \stackrel{\sim}{\longrightarrow}\left(p_{1} \circ s_{n}\right)^{*}\left(\mathcal{B}_{Y}^{\left(n_{m}\right)}\left(H_{Y}\right)\right)$. Via $\delta_{f, f^{\prime}}^{n}{ }^{*}$, on obtient 
$f^{\prime *}\left(\mathcal{B}_{Y}^{\left(n_{m}\right)}\left(H_{Y}\right)\right) \stackrel{\sim}{\longrightarrow} f^{*}\left(\mathcal{B}_{Y}^{\left(n_{m}\right)}\left(H_{Y}\right)\right)$ s'inscrivant dans le diagramme commutatif

$$
f^{\prime *}\left(\mathcal{B}_{Y}^{\left(n_{m}\right)}\left(H_{Y}\right)\right) \longrightarrow f_{\mathcal{B}_{X}^{\left(n_{m}\right)}\left(H_{X}\right),}^{\sim}\left(\mathcal{B}_{Y}^{\left(n_{m}\right)}\left(H_{Y}\right)\right)
$$

dont les flèches obliques sont les morphismes canoniques ([21, 2.1.2]).

Soit $\widetilde{\Delta}_{X / S(m)}^{n}$ l'espace annelé $\left(\Delta_{X / S(m)}^{n}, \mathcal{O}_{\widetilde{\Delta}_{X / S(m)}^{n}}\right)$, avec $\mathcal{O}_{\widetilde{\Delta}_{X / S(m)}^{n}}:=\left(p_{1} \circ\right.$ $\left.s_{n}\right)^{*}\left(\mathcal{B}_{X}^{\left(n_{m}\right)}\left(H_{X}\right)\right)$. On note $\tilde{p}_{1}: \widetilde{\Delta}_{X / S(m)}^{n} \rightarrow \widetilde{X}$ le morphisme induit par l'application continue $p_{1} \circ s_{n}$ et par le morphisme d'anneaux canonique $\left(p_{1} \circ\right.$ $\left.s_{n}\right)^{-1}\left(\mathcal{B}_{X}^{\left(n_{m}\right)}\left(H_{X}\right)\right) \rightarrow \mathcal{O}_{\widetilde{\Delta}_{X / S(m)}^{n}}$ et $\tilde{p}_{2}: \widetilde{\Delta}_{X / S(m)}^{n} \rightarrow \widetilde{X}$ dont le morphisme d'espaces topologiques est $p_{2} \circ s_{n}$ et dont le morphisme d'anneaux est $\left(p_{2} \circ\right.$ $\left.s_{n}\right)^{-1}\left(\mathcal{B}_{X}^{\left(n_{m}\right)}\left(H_{X}\right)\right) \rightarrow\left(p_{2} \circ s_{n}\right)^{*}\left(\mathcal{B}_{X}^{\left(n_{m}\right)}\left(H_{X}\right)\right) \stackrel{\sim}{\longrightarrow}\left(p_{1} \circ s_{n}\right)^{*}\left(\mathcal{B}_{X}^{\left(n_{m}\right)}\left(H_{X}\right)\right)=$ $\mathcal{O}_{\widetilde{\Delta}_{X / S(m)}^{n}}$. De même, lorsque l'on remplace respectivement $S$ et $X$ par $T$ et $Y$ ou $U$ et $Z$.

On désigne par $\delta_{\tilde{f}, \tilde{f}^{\prime}}^{n}: \widetilde{X} \rightarrow \widetilde{\Delta}_{Y / T(m)}^{n}$ le morphisme induit par $\delta_{f, f^{\prime}}^{n}$ et $\operatorname{par} \delta_{f, f^{\prime}}^{n}{ }^{-1}\left(\mathcal{O}_{\widetilde{\Delta}_{Y / T(m)}^{n}}\right) \rightarrow \delta_{f, f^{\prime}}^{n}{ }^{*}\left(\mathcal{O}_{\widetilde{\Delta}_{Y / T(m)}^{n}}\right)=\delta_{f, f^{\prime}}^{n}{ }^{*}\left(p_{1} \circ s_{n}\right)^{*}\left(\mathcal{B}_{Y}^{\left(n_{m}\right)}\left(H_{Y}\right)\right) \stackrel{\sim}{\longrightarrow}$ $f^{*}\left(\mathcal{B}_{Y}^{\left(n_{m}\right)}\left(H_{Y}\right)\right) \rightarrow \mathcal{B}_{X}^{\left(n_{m}\right)}\left(H_{X}\right)$. Via 2.1.1.2, on vérifie $\tilde{f}=\tilde{p}_{1} \delta_{\tilde{f}, \tilde{f}^{\prime}}^{n}$ et $\tilde{f}^{\prime}=$ $\tilde{p}_{2} \delta_{\tilde{f}, \tilde{f}^{\prime}}^{n} \cdot$

Pour tout complexe $\mathcal{F}$ de $\mathcal{B}_{Y}^{\left(n_{m}\right)}\left(H_{Y}\right) \otimes \mathcal{O}_{Y} \mathcal{D}_{Y / T}^{(m)}$-modules borné supérieurement, via le diagramme commutatif suivant valable pour $n$ assez grand

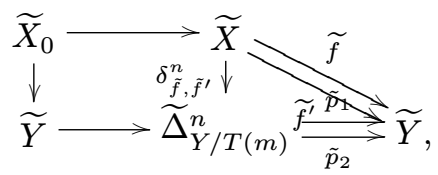

l'isomorphisme $\widetilde{\mathcal{P}}_{Y / T(m)}^{n}$-linéaire $\widetilde{\mathcal{P}}_{Y / T(m)}^{n} \otimes_{\mathcal{B}_{Y}^{\left(n_{m}\right)}\left(H_{Y}\right)} \mathcal{F} \stackrel{\sim}{\longrightarrow} \mathcal{F} \otimes_{\mathcal{B}_{Y}^{\left(n_{m}\right)}\left(H_{Y}\right)}$ $\widetilde{\mathcal{P}}_{Y / T(m)}^{n}$ induit un isomorphisme $\widetilde{\mathcal{D}}_{X / S}^{(m)}$-linéaire canonique fonctoriel en $\mathcal{F}$

$$
\tau_{\tilde{f}, \tilde{f}^{\prime}}^{\mathcal{F}}: \tilde{f}^{\prime} !(\mathcal{F}) \stackrel{\sim}{\longrightarrow} \tilde{f}^{!}(\mathcal{F})
$$

tel que $\tau_{\tilde{f}, \tilde{f}}^{\mathcal{F}}=\mathrm{Id}$, et que, si $\tilde{f}^{\prime \prime}: X \rightarrow Y$ est un morphisme dont la restriction $X_{0} \rightarrow Y$ coïncide avec celle de $f$, on ait la formule de transitivité $\tau_{\tilde{f}, \tilde{f}^{\prime \prime}}^{\mathcal{F}}=$ $\tau_{\tilde{f}, \tilde{f}^{\prime}}^{\mathcal{F}} \circ \tau_{\tilde{f}^{\prime}, \tilde{f}^{\prime \prime}}^{\mathcal{F}}$. Si aucune confusion n'est à craindre, nous noterons simplement $\tau_{\tilde{f}, \tilde{f}^{\prime}}$ ou $\tau^{\mathcal{F}}$ voire $\tau$.

TOME $137-2009-\mathrm{N}^{\mathrm{O}} 4$ 
2.1.2 (Changement de niveau). - Soient $n_{m^{\prime}} \geq m^{\prime} \geq m$ deux entiers, $\widetilde{\mathcal{D}}_{Y / T}^{\left(m^{\prime}\right)}=\mathcal{B}_{Y}^{\left(n_{m^{\prime}}\right)}\left(H_{Y}\right) \otimes_{\mathcal{O}_{Y}} \mathcal{D}_{Y / T}^{\left(m^{\prime}\right)}$ et $\mathcal{F}$ un complexe de $\widetilde{\mathcal{D}}_{Y / T}^{\left(m^{\prime}\right)}$-modules borné supérieurement. Lorsque $Y$ a des coordonnées locales $t_{1}, \ldots t_{d}$, avec les notations de $[4,2.1 .2]$, le morphisme canonique $\mathcal{P}_{X / S\left(m^{\prime}\right)}^{n} \rightarrow \mathcal{P}_{X / S(m)}^{n}$ envoie $\underline{\tau}^{\{\underline{k}\}_{\left(m^{\prime}\right)}}$ sur $\underline{q} ! / \underline{q}^{\prime} ! \underline{\tau}^{\{\underline{k}\}_{(m)}}$ tandis que le morphisme $\mathcal{D}_{Y / T}^{(m)} \rightarrow \mathcal{D}_{Y / T}^{\left(m^{\prime}\right)}$ envoie $\underline{\partial}^{<\underline{k}>_{(m)}}$

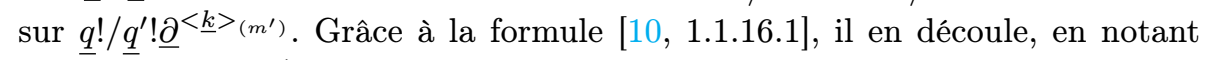
$\operatorname{oub}_{m, m^{\prime}}: D\left(\widetilde{\mathcal{D}}_{Y / T}^{\left(m^{\prime}\right)}\right) \rightarrow D\left(\widetilde{\mathcal{D}}_{Y / T}^{(m)}\right)$ le foncteur oubli, la formule :

$$
\tau^{\mathrm{oub}_{m, m^{\prime}}(\mathcal{F})}=\operatorname{oub}_{m, m^{\prime}} \tau^{\mathcal{F}}
$$

\subsection{3 (Compatibilité aux images inverses extraordinaires)}

Soit $g^{\prime}: Y \rightarrow Z$, un second $U$-morphisme ayant la même restriction $Y_{0} \rightarrow Z$ que $g$. Pour $n$ assez grand, il découle respectivement du diagramme commutatif de droite et de gauche suivant
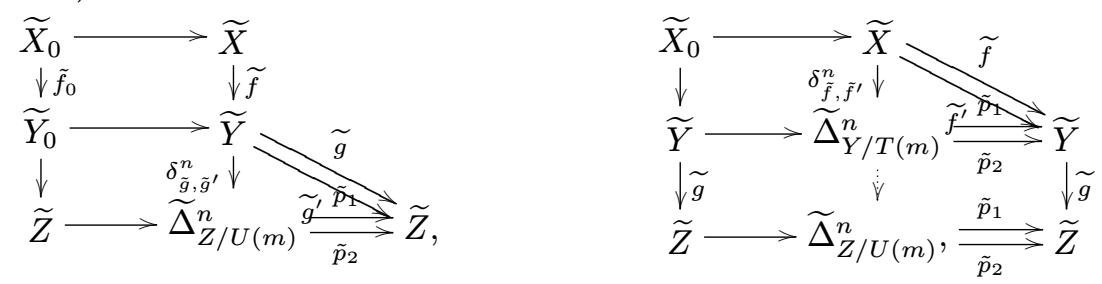

que l'on dispose de l'égalité $\tau_{\tilde{g} \circ \tilde{f}, \tilde{g} \circ \tilde{f}^{\prime}}=\tau_{\tilde{f}, \tilde{f}^{\prime}} \circ \tilde{g}^{!}$et $\tilde{f}^{!} \circ \tau_{\tilde{g}, \tilde{g}^{\prime}}=\tau_{\tilde{g} \circ \tilde{f}, \tilde{g}^{\prime} \circ \tilde{f}}$.

Ainsi, les isomorphismes $\tau$ sont compatibles avec la composition des images inverses.

2.1.4 (Changement de base). - Considérons le diagramme commutatif

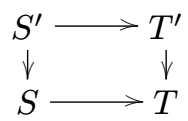

de $m$-PD-morphismes. On note $X^{\prime}=X \times_{S} S^{\prime}, Y^{\prime}=Y \times_{T} T^{\prime}, g$ et $g^{\prime}: X^{\prime} \rightarrow Y^{\prime}$ les morphismes induits respectivement par $f$ et $f^{\prime}$. Il découle de la propriété universelle des enveloppes à puissances divisées partielles $([4,1.4 .1])$ que l'on dispose d'un morphisme $\Delta_{Y^{\prime} / T^{\prime}(m)}^{n} \rightarrow \Delta_{Y / T(m)}^{n}$ rendant commutatif le diagramme

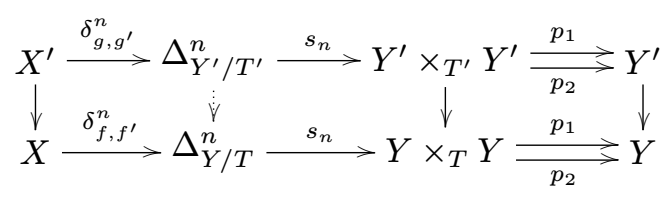


En notant respectivement $H_{X^{\prime}}$ et $H_{Y^{\prime}}$ les images inverses de $H_{X}$ et $H_{Y}, \widetilde{X}^{\prime}=$ $\left(X^{\prime}, \mathcal{B}_{X}^{\left(n_{m}\right)}\left(H_{X^{\prime}}\right)\right)$ et $\widetilde{Y}^{\prime}=\left(Y^{\prime}, \mathcal{B}_{Y}^{\left(n_{m}\right)}\left(H_{Y^{\prime}}\right)\right)$, les morphismes $g$ et $g^{\prime}$ induisent canoniquement des morphismes $\widetilde{X}^{\prime} \rightarrow \widetilde{Y}^{\prime}$ notés $\tilde{g}$ et $\tilde{g}^{\prime}$. On déduit ensuite de la commutativité du diagramme 2.1.4.1 celle de

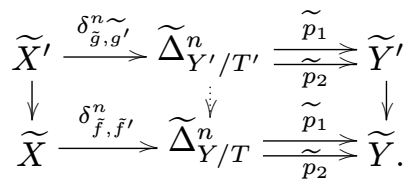

Il en résulte, notant $\theta: \widetilde{X}^{\prime} \rightarrow \widetilde{X}$ le morphisme canonique, $\tau_{\tilde{f}, \tilde{f}^{\prime}} \stackrel{\sim}{\longrightarrow} \tau_{\tilde{g}, \tilde{g}^{\prime}}$. Autrement dit, les isomorphismes de la forme $\tau$ sont compatibles aux changements de base.

Remarques 2.1.5. - Avec les notations de 2.1.1, soit $s$ le morphisme canonique $\Delta_{Y / T} \rightarrow Y \times_{T} Y$. On obtient $\widetilde{\Delta}_{Y / T(m)}$ l'espace annelé $\left(\Delta_{Y / T(m)}, \mathcal{O}_{\widetilde{\Delta}_{Y / T(m)}}\right)$ en posant $\mathcal{O}_{\widetilde{\Delta}_{Y / T(m)}}:=\left(p_{1} \circ s\right)^{*}\left(\mathcal{B}_{Y}^{\left(n_{m}\right)}\left(H_{Y}\right)\right)$. De même, on construit $\delta_{\tilde{f}, \tilde{f}^{\prime}}$ : $\widetilde{X} \rightarrow \widetilde{\Delta}_{Y / T(m)}$, celui-ci s'insérant dans le diagramme commutatif 2.1.1.3 où on a enlevé les indices $n$. Si $p$ est localement nilpotent sur $U$ (et donc sur $T$ et $S$ ), pour tout complexe quasi-nilpotent (voir 2.4.4 et 2.4.5) $\mathcal{F}$ de $\mathcal{B}_{Y}^{\left(n_{m}\right)}\left(H_{Y}\right) \otimes_{\mathcal{O}_{Y}} \mathcal{D}_{Y / T}^{(m)}$-modules borné supérieurement, on en déduit, de manière analogue à ceux de 2.1.1, des isomorphismes $\tau_{\tilde{f}, \tilde{f}^{\prime}}^{\mathcal{F}}$, ceux-ci vérifiant les conditions de transitivité, de changement de base et de commutation à la composition des images inverses.

2.1.6. - Avec les notations et hypothèses de 2.1.1 (nous omettons d'indiquer la base), on pose

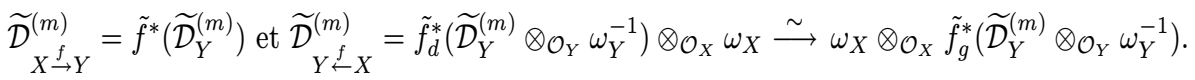

Supposons $S=T, S$ noethérien et de dimension de Krull finie, $f$ et $f^{\prime}$ quasi-

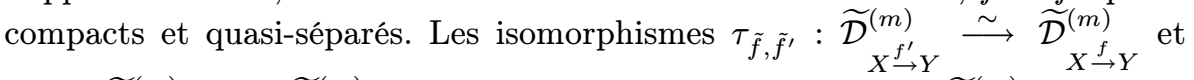

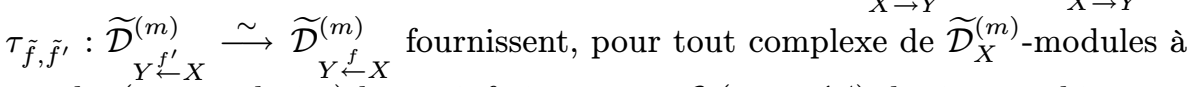
gauche (resp. à droite) borné inférieurement $\mathcal{E}$ (resp. $\mathcal{M})$, les isomorphismes

$\sigma_{\tilde{f}, \tilde{f}^{\prime}}: f_{H_{Y}, H_{X}+}^{\prime}(\mathcal{E}) \stackrel{\sim}{\longrightarrow} f_{H_{Y}, H_{X}+}(\mathcal{E})$ et $\left.\sigma_{\tilde{f}, \tilde{f}^{\prime}}: f_{H_{Y}, H_{X}+}^{\prime}(\mathcal{M}) \stackrel{\sim}{\longrightarrow} f_{H_{Y}, H_{X}+}(\mathcal{M})\right)$.

De plus, pour tout morphisme $\tilde{f}^{\prime \prime}: X \rightarrow Y$ dont la restriction $X_{0} \rightarrow Y$ coïncide avec celle de $f$, on a la formule de transitivité $\sigma_{\tilde{f}, \tilde{f}^{\prime \prime}}=\sigma_{\tilde{f}, \tilde{f}^{\prime}} \circ \sigma_{\tilde{f}^{\prime}, \tilde{f}^{\prime \prime}}$. 
REMARQUeS 2.1.7. - Soient $\mathcal{F} \in D^{-}\left(\widetilde{\mathcal{D}}_{Y}^{(m)}\right), \tilde{p}_{1 *} \widetilde{\mathcal{P}}_{Y(m)}^{n}\left(\right.$ resp. $\left.\tilde{p}_{2 *} \widetilde{\mathcal{P}}_{Y(m)}^{n}\right)$ le faisceau $\widetilde{\mathcal{P}}_{Y(m)}^{n}$ vu comme $\mathcal{B}_{Y}^{(m)}\left(H_{Y}\right)$-algèbre pour la structure gauche (resp. droite). Considérons les diagrammes

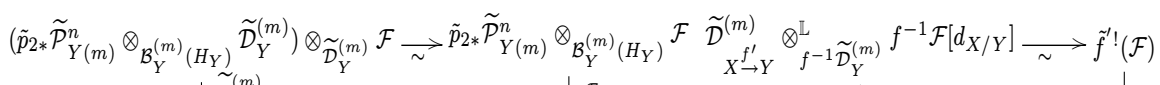

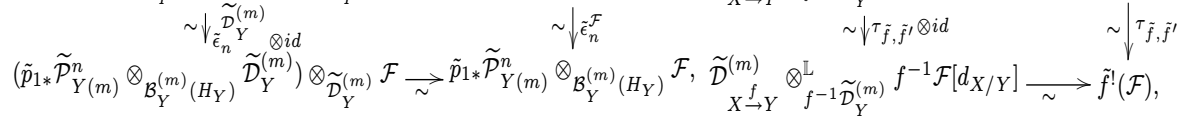

où $\tilde{\epsilon}_{n}$ désignent les $m$-PD-stratifications relatives à $\mathcal{B}_{Y}^{(m)}\left(H_{Y}\right)$ (voir [10, 1.1]). Via un calcul utilisant la formule [10, 1.1.16.1], on obtient la commutativité du diagramme de gauche. Il en résulte celle de droite.

LEMME 2.1.8. - Avec les notations de 2.1.1, les isomorphismes

$$
\begin{aligned}
& \widetilde{\mathcal{D}}_{X \rightarrow Z}^{(m)} \stackrel{\sim}{\longrightarrow} \widetilde{\mathcal{D}}_{X \rightarrow Y}^{(m)} \otimes_{f^{-1}} \widetilde{\mathcal{D}}_{Y}^{(m)} f^{-1} \widetilde{\mathcal{D}}_{Y \rightarrow Z}^{(m)} \stackrel{\sim}{\longrightarrow} \widetilde{\mathcal{D}}_{X \rightarrow Y}^{(m)} \otimes_{f^{-1} \widetilde{\mathcal{D}}_{Y}^{(m)}}^{\mathbb{L}} f^{-1} \widetilde{\mathcal{D}}_{Y \rightarrow Z}^{(m)} \\
& \widetilde{\mathcal{D}}_{Z \leftarrow X}^{(m)} \stackrel{\sim}{\longrightarrow} f^{-1} \widetilde{\mathcal{D}}_{Z \leftarrow Y}^{(m)} \otimes_{f^{-1} \widetilde{\mathcal{D}}_{Y}^{(m)}} \widetilde{\mathcal{D}}_{Y \leftarrow X}^{(m)} \stackrel{\sim}{\longrightarrow} f^{-1} \widetilde{\mathcal{D}}_{Z \leftarrow Y}^{(m)} \otimes_{f^{-1}}^{\mathbb{L}} \widetilde{\mathcal{D}}_{Y}^{(m)} \widetilde{\mathcal{D}}_{Y \leftarrow X}^{(m)}
\end{aligned}
$$

sont compatibles aux isomorphismes de la forme $\tau$.

Démonstration. - La construction des isomorphismes est analogue à [7, VI.6.3]. Pour la première ligne, la compatibilité aux isomorphismes $\tau$ est une conséquence de la remarque 2.1.7 et des formules $\tau_{\tilde{g} \circ \tilde{f}, \tilde{g} \circ \tilde{f}^{\prime}}=\tau_{\tilde{f}, \tilde{f}^{\prime}} \circ \tilde{g}^{!}$et $\tilde{f}^{!} \circ \tau_{\tilde{g}, \tilde{g}^{\prime}}=\tau_{\tilde{g} \circ \tilde{f}, \tilde{g}^{\prime} \circ \tilde{f}}$ prouvées via les diagrammes 2.1.3.1. Ceux de la deuxième ligne s'en déduisent.

Proposition 2.1.9. - Avec les notations de 2.1.1, on suppose $S=T=U$, $S$ noethérien, de dimension de Krull finie, $f, f^{\prime}, g$ et $g^{\prime}$ quasi-compacts et quasi-séparés. Les isomorphismes de la forme $\sigma$ sont alors compatibles à la composition des images directes, i.e., $g_{H_{Z}, H_{Y}+} \circ \sigma_{f, f^{\prime}}=\sigma_{g \circ f, g \circ f^{\prime}}$ et $\sigma_{g, g^{\prime}} \circ$ $f_{H_{Y}, H_{X}+}=\sigma_{g \circ f, g^{\prime} \circ f}$.

Démonstration. - La commutation à la composition des foncteurs images directes se prouve de manière analogue à [7, VI.6.4]. Par construction de celle-ci, cela découle du lemme 2.1.8.

2.1.10. - Maintenant, soient $f: \mathfrak{X} \rightarrow \mathcal{Y}$ un morphisme de $\mathcal{V}$-schémas formels lisses, $T_{Y}$ un diviseur de $Y, T_{X} \supset f_{0}^{-1}\left(T_{Y}\right)$ un diviseur de $X$. On se donne un deuxième morphisme $f^{\prime}: \mathfrak{X} \rightarrow \mathcal{Y}$ tel que $f_{0}^{\prime}=f_{0}$. Donnonsnous une suite croissante d'entiers $\left(n_{m}\right)_{m \in \mathbb{N}}$ telle que $n_{m} \geq m$ et posons $\widehat{\mathcal{D}}_{\mathfrak{X}}^{(m)}\left(T_{X}\right):=\mathcal{B}_{\mathfrak{X}}^{\left(n_{m}\right)}\left(T_{X}\right) \widehat{\otimes}_{\mathcal{O}_{\mathfrak{X}}} \mathcal{D}_{\mathfrak{X}}^{(m)}$. De même, en remplaçant $\mathfrak{X}$ par $\mathcal{Y}$. Comme pour $[6,3.2 .1]$, on définit la catégorie des complexes de $\widehat{\mathcal{D}}_{\mathfrak{X}}^{(m)}\left(T_{X}\right)$ modules à gauche à cohomologie bornée et quasi-cohérente et qui sera noté 
$D_{\mathrm{qc}}^{\mathrm{b}}\left(\widehat{\mathcal{D}}_{\mathfrak{X}}^{(m)}\left(T_{X}\right)\right)$. Avec les notations [12, 1.1.6,1.1.7], on dispose des foncteurs images inverses extraordinaires de niveau $m$ (définis comme dans [6, 3.4.2.1]): $f_{T_{X}, T_{Y}}^{!(m)}$ et $f_{T_{X}, T_{Y}}^{\prime !(m)}: D_{\mathrm{qc}}^{\mathrm{b}}\left(\widehat{\mathcal{D}}_{\mathcal{Y}}^{(m)}\left(T_{Y}\right)\right) \rightarrow D_{\mathrm{qc}}^{\mathrm{b}}\left(\widehat{\mathcal{D}}_{\mathfrak{X}}^{(m)}\left(T_{X}\right)\right)$. Les isomorphismes de la forme $\tau$ (voir 2.1.1) commutant aux changements de base, on obtient, pour tout $\mathcal{F} \in D_{\mathrm{qc}}^{\mathrm{b}}\left(\widehat{\mathcal{D}}_{\mathcal{Y}}^{(m)}\left(T_{Y}\right)\right)$, des isomorphismes $\tau_{f, f^{\prime}, T_{X}, T_{Y}}^{\mathcal{F}(m)}$ : $f_{T_{X}, T_{Y}}^{\prime !(m)}(\mathcal{F}) \stackrel{\sim}{\longrightarrow} f_{T_{X}, T_{Y}}^{!(m)}(\mathcal{F})$. En procédant à deux localisations (correspondant à la tensorisation par $\mathbb{Q}$ et au passage à la limite sur le niveau), on obtient de manière analogue à $[6,4.2 .1]$ les catégories de la forme $L D_{\mathbb{Q}, \mathrm{qc}}^{\mathrm{b}}\left(\widehat{\mathcal{D}}_{\mathcal{Y}}^{(\bullet)}\left(T_{Y}\right)\right)$ (ces dernières ne dépendent pas du choix de la suite d'entiers $\left(n_{m}\right)_{m \in \mathbb{N}}$ ). Il découle des isomorphismes $\tau_{f, f^{\prime}, T_{X}, T_{Y}}^{\mathcal{F}(m)}$ avec $m$ variable, pour tout objet $\mathcal{F}$ de $\underset{\sim}{L} D_{\mathbb{Q}, \mathrm{qc}}^{\mathrm{b}}\left(\widehat{\mathcal{D}}_{\mathcal{Y}}^{(\bullet)}\left(T_{Y}\right)\right)$, le suivant $\tau_{f, f^{\prime}, T_{X}, T_{Y}}^{\mathcal{F}}: f_{T_{X}, T_{Y}}^{\prime}(\mathcal{F}) \stackrel{\sim}{\longrightarrow} f_{T_{X}, T_{Y}}^{!}(\mathcal{F})$, où $f_{T_{X}, T_{Y}}^{!}$et $f_{T_{X}, T_{Y}}^{\prime}$ sont les foncteurs images inverses extraordinaires définis comme pour [6, 4.3.2.1]. On pourra le noter plus simplement $\tau_{f, f^{\prime}}$ ou $\tau^{\mathcal{F}}$

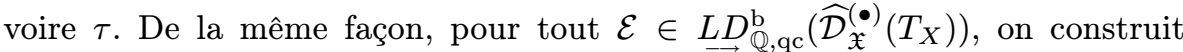
l'isomorphisme $f_{T_{Y}, T_{X}+}^{\prime}(\mathcal{E}) \stackrel{\sim}{\longrightarrow} f_{T_{Y}, T_{X}+}(\mathcal{E})$, que l'on notera $\sigma_{f, f^{\prime}, T_{X}, T_{Y}}$ (ou $\sigma_{f, f^{\prime}}$ voire $\sigma$ si aucune confusion n'est à craindre). Les isomorphismes de la forme $\tau$ (resp. $\sigma$ ) vérifient aussi la formule de transitivité et celle de leur commutation à l'image inverse extraordinaire (resp. image directe) décrites ci-dessus dans le cas des schémas.

Proposition 2.1.11. - Avec les notations de 2.1.10, pour tout objet $\mathcal{F}$ de $L D_{\mathbb{Q}, \mathrm{qc}}^{\mathrm{b}}\left(\widehat{\mathcal{D}}_{\mathcal{Y}}^{(\bullet)}\right)$, on a les diagrammes commutatifs :

$$
\begin{aligned}
& f_{T_{X}, T_{Y}}^{!}\left({ }^{\dagger} T_{Y}\right)(\mathcal{F}) \longrightarrow\left({ }^{\dagger} T_{X}\right) f^{!}(\mathcal{F}) \\
& \tau_{f, f^{\prime}, T_{X}, T_{Y}}^{\left(\dagger T_{X}\right)(\mathcal{F})} \uparrow \sim\left({ }^{\dagger} T_{X}\right)\left(\tau_{f, f^{\prime}}^{\mathcal{F}}\right) \uparrow \sim \\
& f_{T_{X}, T_{Y}}^{\prime \prime{ }^{\prime}}\left({ }^{\dagger} T_{Y}\right)(\mathcal{F}) \longrightarrow\left({ }^{\dagger} T_{X}\right) f^{\prime !}(\mathcal{F}), \\
& \begin{array}{c}
f^{!}\left({ }^{\dagger} T_{Y}\right)(\mathcal{F}) \underset{\left.{ }^{\dagger} T_{Y}\right)(\mathcal{F}) \uparrow \sim}{\sim} \underset{\left.{ }^{\dagger} T_{X}\right)\left({ }^{\dagger} \tau_{f, f^{\prime}}\right) \uparrow \sim}{\longrightarrow}\left(T_{X}\right) f^{!}(\mathcal{F}) \\
f^{\prime \prime}\left({ }^{\dagger} T_{Y}\right)(\mathcal{F}) \underset{\sim}{\longrightarrow}\left({ }^{\dagger} T_{X}\right) f^{\prime !}(\mathcal{F}) .
\end{array}
\end{aligned}
$$

Démonstration. - Notons $f_{i}$ et $f_{i}^{\prime}: X_{i} \rightarrow Y_{i}$ les réductions modulo $\mathfrak{m}^{i+1}$ de $f$ et $f^{\prime}$, et $\tilde{f}_{i}$ et $\tilde{f}_{i}^{\prime}$ les morphismes d'espaces annelés canoniques $\left(X_{i}, \mathcal{B}_{X_{i}}^{\left(n_{m}\right)}\left(T_{X}\right)\right) \rightarrow\left(Y_{i}, \mathcal{B}_{Y_{i}}^{\left(n_{m}\right)}\left(T_{Y}\right)\right)$ induits par $f$ et $f^{\prime}$. Pour tout $\mathcal{F}_{i} \in D^{-}\left(\mathcal{B}_{Y_{i}}^{\left(n_{m}\right)}\left(T_{Y}\right) \otimes_{\mathcal{O}_{Y_{i}}} \mathcal{D}_{Y_{i}}^{(m)}\right)$, le diagramme

$$
\begin{gathered}
\tilde{f}_{i}^{!}\left(\mathcal{B}_{Y_{i}}^{\left(n_{m}\right)}\left(T_{Y}\right) \otimes_{\mathcal{O}_{Y_{i}}}^{\mathbb{L}} \mathcal{F}_{i}\right) \longrightarrow \mathcal{B}_{X_{i}}^{\left(n_{m}\right)}\left(T_{X}\right) \otimes_{\mathcal{O}_{X_{i}}}^{\mathbb{L}} f_{i}^{!}\left(\mathcal{F}_{i}\right) \\
{\tilde{\tau_{f}, \tilde{f}_{i}^{\prime} \uparrow}}_{i d \otimes \tau_{f_{i}, f_{i}^{\prime} \uparrow}} \\
\tilde{f}_{i}^{\prime !}\left(\mathcal{B}_{Y_{i}}^{\left(n_{m}\right)}\left(T_{Y}\right) \otimes_{\mathcal{O}_{Y_{i}}}^{\mathbb{L}} \mathcal{F}_{i}\right) \longrightarrow \mathcal{B}_{X_{i}}^{\left(n_{m}\right)}\left(T_{X}\right) \otimes_{\mathcal{O}_{X_{i}}}^{\mathbb{L}} f_{i}^{\prime !}\left(\mathcal{F}_{i}\right)
\end{gathered}
$$

est commutatif. Il en résulte celle du diagramme de gauche de 2.1.11. De plus, comme chacune des flèches du morphisme composé $f^{!}\left({ }^{\dagger} T_{Y}\right)(\mathcal{F}) \stackrel{\sim}{\longrightarrow}$ 
$\left({ }^{\dagger} T_{X}\right) f^{!}\left({ }^{\dagger} T_{Y}\right)(\mathcal{F}) \tilde{\leftarrow}\left({ }^{\dagger} T_{X}\right) f^{!}(\mathcal{F})$, qui correspond à la flèche du haut du diagramme de droite de 2.1.11, commutent par fonctorialité aux isomorphismes $\tau$, on obtient la commutativité de celui de droite.

Remarques 2.1.12. - Avec les notations de 2.1.11, supposons $f^{-1}\left(T_{Y}\right)=$ $T_{X}$. L'isomorphisme $f_{T_{Y}}^{!} \stackrel{\sim}{\longrightarrow} f^{!}$(on a omis d'indiquer les foncteurs oublis) commute alors aux isomorphismes de recollements $\tau$. En effet, celui-ci est construit de la façon suivante :

$f_{T_{Y}}^{!}\left(\left({ }^{\dagger} T_{Y}\right) \mathcal{F}\right) \stackrel{\sim}{\longrightarrow} f_{T_{Y}}^{!}\left({ }^{\dagger} T_{Y}\right)\left(\left({ }^{\dagger} T_{Y}\right) \mathcal{F}\right) \stackrel{\sim}{\longrightarrow}\left({ }^{\dagger} T_{X}\right) f^{!}\left(\left({ }^{\dagger} T_{Y}\right) \mathcal{F}\right) \stackrel{\sim}{\longrightarrow} f^{!}\left({ }^{\dagger} T_{Y}\right)\left(\left({ }^{\dagger} T_{Y}\right) \mathcal{F}\right) \tilde{\leftarrow} f^{!}\left(\left({ }^{\dagger} T_{Y}\right) \mathcal{F}\right)$.

\subsection{Morphisme d'adjonction associé à un carré}

2.2.1. - Soit le diagramme de $\mathcal{V}$-schémas formels lisses :

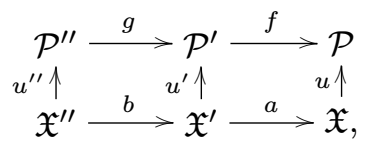

où $f, g, a$ et $b$ sont lisses et où $u, u^{\prime}$ et $u^{\prime \prime}$ sont des immersions fermées. On suppose que le diagramme 2.2.1.1 est commutatif au niveau des fibres spéciales (mais non nécessairement commutatif au niveau des schémas formels). De plus, on se donne $T_{P}$ un diviseur de $P$ tel que $T_{P^{\prime}}:=f^{-1}\left(T_{P}\right)$ (resp. $T_{P^{\prime \prime}}:=g^{-1}\left(T_{P^{\prime}}\right), T_{X}:=u^{-1}\left(T_{P}\right), T_{X^{\prime}}:=u^{\prime-1}\left(T_{P^{\prime}}\right)$ et $\left.T_{X^{\prime \prime}}:=u^{\prime \prime-1}\left(T_{P^{\prime \prime}}\right)\right)$ soit un diviseur de $P^{\prime}$ (resp. $P^{\prime \prime}, X, X^{\prime}$ et $\left.X^{\prime \prime}\right)$.

Proposition 2.2.2. - Avec les notations de 2.2.1, on dispose d'un morphisme, dit d'adjonction, fonctoriel en $\mathcal{E} \in D_{\text {coh }}^{\mathrm{b}}\left(\mathcal{D}_{\mathfrak{X}}^{\dagger}\left({ }^{\dagger} T_{X}\right)_{\mathbb{Q}}\right), \phi(\mathcal{E})$ : $u_{+}^{\prime} \circ a^{!}(\mathcal{E}) \rightarrow f^{!} \circ u_{+}(\mathcal{E})$. Si la réduction au niveau des fibres spéciales $d u$ carré de droite de 2.2.1.1 est cartésien, alors $\phi(\mathcal{E})$ est un isomorphisme. Le morphisme d'adjonction entre foncteurs, $u_{+}^{\prime} \circ a^{!} \rightarrow f^{!} \circ u_{+}$, sera noté $\phi$.

(i) En notant $\phi^{\prime}: u_{+}^{\prime \prime} \circ b^{!} \rightarrow g^{!} \circ u_{+}^{\prime}\left(\right.$ resp. $\left.\phi^{\prime \prime}: u_{+}^{\prime \prime} \circ(a \circ b)^{!} \rightarrow(f \circ g)^{!} \circ u_{+}\right) l e$ morphisme d'adjonction du carré de gauche de 2.2.1.1 (resp. du grand rectangle de 2.2.1.1), le diagramme

$$
\begin{gathered}
u_{+}^{\prime \prime} \circ(a \circ b)^{!} \longrightarrow u_{+}^{\prime \prime} \circ b^{!} \circ a^{!} \\
\downarrow \phi^{\prime \prime} \\
\left.(f \circ g)^{!} \circ u_{+} \longrightarrow g^{!} \circ \phi\right) \circ\left(\phi^{\prime} \circ a^{!}\right) \\
\sim g^{!} \circ f^{!} \circ u_{+},
\end{gathered}
$$

est alors commutatif. Ainsi, avec des abus de notations, on dispose de la formule de transitivité $\phi^{\prime \prime}=\left(g^{!} \circ \phi\right) \circ\left(\phi^{\prime} \circ a^{!}\right)$(pour le composé de deux morphismes lisses) de l'isomorphisme de changement de base par un morphisme lisse de l'image directe par une immersion fermée. 
(ii) Soit $a^{\prime}: \mathfrak{X}^{\prime} \rightarrow \mathfrak{X}$ (resp. $f^{\prime}: \mathcal{P}^{\prime} \rightarrow \mathcal{P}$ ) un morphisme dont la réduction $X^{\prime} \rightarrow \mathfrak{X}\left(\right.$ resp. $\left.P^{\prime} \rightarrow \mathcal{P}\right)$ coïncide avec celle de a (resp. f). Le diagramme ci-dessous

$$
\begin{aligned}
& u_{+}^{\prime} a^{!} \stackrel{\phi}{\longrightarrow} f^{!} \circ u_{+} \\
& u_{+}^{\prime}\left(\tau_{a, a^{\prime}}\right) \uparrow \sim \tau_{f, f^{\prime}} u_{+} \uparrow \sim \\
& u_{+}^{\prime} a^{\prime !} \stackrel{\psi}{\longrightarrow} f^{\prime !} \circ u_{+},
\end{aligned}
$$

où $\psi$ désigne le morphisme d'adjonction du diagramme de droite de 2.2.1.1 lorsque a et $f$ ont été remplacés respectivement par $a^{\prime}$ et $f^{\prime}$, est commutatif.

Démonstration. - Construisons d'abord le morphisme $\phi(\mathcal{E})$. Comme $\mathcal{E} \in$ $D_{\text {coh }}^{\mathrm{b}}\left(\mathcal{D}_{\mathfrak{X}}^{\dagger}\left({ }^{\dagger} T_{X}\right)_{\mathbb{Q}}\right)$, en appliquant le foncteur $u_{+}^{\prime} a^{!}$au morphisme d'adjonction de $u$ en $\mathcal{E}([12,1.2 .11])$, on obtient : $u_{+}^{\prime} a^{!}(\mathcal{E}) \rightarrow u_{+}^{\prime} a^{!} u^{!} u_{+}(\mathcal{E})$. Or, comme $\left(f \circ u^{\prime}\right)^{!} \stackrel{\sim}{\longrightarrow} u^{\prime !} f^{!}$et $(u \circ a)^{!} \stackrel{\sim}{\longrightarrow} a^{!} u^{!}$, on a l'isomorphisme $u_{+}^{\prime} \tau_{f \circ u^{\prime}, u \circ a} u_{+}(\mathcal{E})$ : $u_{+}^{\prime} a^{!} u^{!} u_{+}(\mathcal{E}) \stackrel{\sim}{\longrightarrow} u_{+}^{\prime} u^{\prime !} f^{!} u_{+}(\mathcal{E})$ (notations de 2.1.10). Ensuite, puisque $f^{!} u_{+}(\mathcal{E}) \in D_{\text {coh }}^{\mathrm{b}}\left(\mathcal{D}_{\mathcal{P}^{\prime}}^{\dagger}\left({ }^{\dagger} T_{P^{\prime}}\right)_{\mathbb{Q}}\right)$ et $u^{\prime !} f^{!} u_{+}(\mathcal{E}) \in D_{\text {coh }}^{\mathrm{b}}\left(\mathcal{D}_{\mathfrak{X}^{\prime}}^{\dagger}\left({ }^{\dagger} T_{X^{\prime}}\right)_{\mathbb{Q}}\right)$, il résulte de $[12,1.2 .12]$ que l'on dispose du morphisme d'adjonction de $u^{\prime}$ en $f^{!} u_{+}(\mathcal{E})$ : $u_{+}^{\prime} u^{\prime !} f^{!} u_{+}(\mathcal{E}) \rightarrow f^{!} u_{+}(\mathcal{E})$. En composant ces trois morphismes, il vient : $\phi(\mathcal{E}):=u_{+}^{\prime} a^{!}(\mathcal{E}) \rightarrow f^{!} u_{+}(\mathcal{E})$.

À présent, établissons que le morphisme $\phi(\mathcal{E})$ est un isomorphisme lorsque le diagramme de droite de 2.2.1.1 est cartésien. D'abord, comme $u$ est une immersion fermée, la première flèche dans la construction de $\phi(\mathcal{E})$ est un isomorphisme. En outre, l'hypothèse cartésienne implique que le faisceau $f^{!} u_{+}(\mathcal{E})$ est à support dans $X^{\prime}$ et donc que le morphisme d'adjonction de $u^{\prime}$ en $f^{!} u_{+}(\mathcal{E})$ est un isomorphisme (voir [12, 1.2.12]).

Prouvons à présent la formule de transitivité de (i). Pour cela, considérons le diagramme suivant :

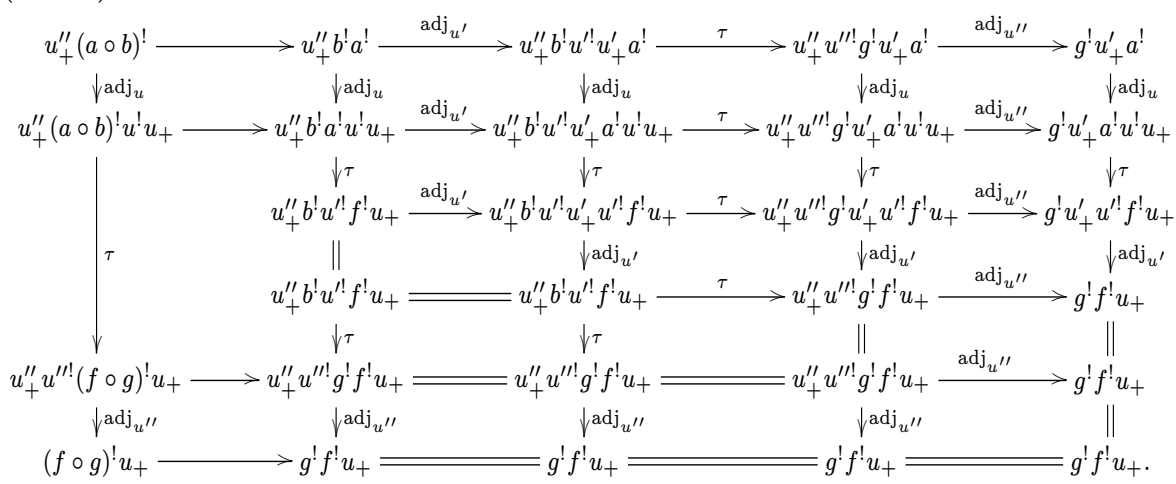

TOME $137-2009-\mathrm{N}^{\mathrm{O}} 4$ 
La commutativité du rectangle (le «seul » rectangle : à gauche et au milieu) de 2.2.2.1 se déduit de toutes les propriétés, données dans 2.1.10, des isomorphismes de la forme $\tau$, ainsi que du diagramme suivant

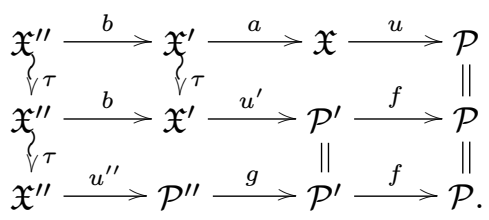

De plus, on remarque que le morphisme composé $u^{\prime !} \stackrel{\operatorname{adj}_{u^{\prime}}}{\longrightarrow} u^{\prime !} u_{+}^{\prime} u^{\prime !} \stackrel{\operatorname{adj}_{u^{\prime}}}{\longrightarrow} u^{\prime}$ est l'identité. En effet, cela résulte de l'isomorphisme d'adjonction de bifoncteurs $\theta: \operatorname{Hom}_{\mathcal{D}_{\mathcal{P}^{\prime}}^{\dagger}\left(\dagger T_{P^{\prime}}\right)}\left(u_{+}^{\prime}(-),-\right) \stackrel{\sim}{\longrightarrow} \operatorname{Hom}_{\mathcal{D}_{\mathfrak{X}^{\prime}}^{\dagger}\left(\dagger T_{X^{\prime}}\right)}\left(-, u^{\prime !}(-)\right)$ (on n'utilisera que la fonctorialité à droite), et du fait que le morphisme identité $u_{+}^{\prime} u^{\prime !} \stackrel{\mathrm{Id}}{\longrightarrow} u_{+}^{\prime} u^{\prime !}$ s'envoie via $\theta$ sur $u^{\prime !} \stackrel{\operatorname{adj}_{u^{\prime}}}{\longrightarrow} u^{\prime !} u_{+}^{\prime} u^{\prime !}$ tandis que $u_{+}^{\prime} u^{\prime !} \stackrel{\operatorname{adj}_{u^{\prime}}}{\longrightarrow}$ Id s'envoie sur $u^{\prime !} \stackrel{\mathrm{Id}}{\longrightarrow}$ $u^{\prime}$. Il en résulte la commutativité du carré de gauche de la troisième ligne de 2.2.2.1.

On vérifie ensuite, par définition ou par fonctorialité, la commutativité des autres carrés de 2.2.2.1. Ce diagramme est donc commutatif.

Or, on constate que le morphisme composé de gauche de 2.2.2.1, $u_{+}^{\prime \prime} \circ(a \circ$ $b)^{!} \stackrel{\sim}{\longrightarrow}(f \circ g)^{!} \circ u_{+}$, n'est autre que $\phi^{\prime \prime}$, tandis que celui construit en prenant le chemin qui passe par le haut puis par la droite du contour de 2.2.2.1, $u_{+}^{\prime \prime} \circ$ $b^{!} \circ a^{!} \stackrel{\sim}{\longrightarrow} g^{!} \circ f^{!} \circ u_{+}$, correspond à $\left(g^{!} \circ \phi\right) \circ\left(\phi^{\prime} \circ a^{!}\right)$. D’où (i).

Démontrons maintenant (ii). Grâce à 2.1.10, le diagramme ci-après

$$
\begin{array}{r}
u_{+}^{\prime} a^{!}(\mathcal{E}) \stackrel{\operatorname{adj}_{u}}{\longrightarrow} u_{+}^{\prime} a^{!} u^{!} u_{+}(\mathcal{E}) \stackrel{u_{+}^{\prime} \tau_{f \circ u^{\prime}, u \circ a} u_{+}}{\longrightarrow} u_{+}^{\prime} u^{\prime !} f^{!} u_{+}(\mathcal{E}) \stackrel{\operatorname{adj}_{u^{\prime}}}{\longrightarrow} f^{!} u_{+}(\mathcal{E}) \\
u_{+}^{\prime}\left(\tau_{a, a^{\prime}}\right) \uparrow \\
u_{+}^{\prime} a^{\prime !}(\mathcal{E}) \underset{u_{u \circ a, u \circ a^{\prime}} u_{+} \uparrow}{\longrightarrow} \underset{\operatorname{adj}_{u}}{\longrightarrow} u_{+}^{\prime} a^{\prime !} u^{!} u_{+}(\mathcal{E}) \underset{u_{+}^{\prime} \tau_{f \circ u^{\prime}, f^{\prime} \circ u^{\prime} u^{\prime}, u \circ a^{\prime}} u_{+}}{\longrightarrow} u_{+}^{\prime} u_{f, f^{\prime}} u_{+}{ }^{\prime} f^{\prime !} u_{+}(\mathcal{E}) \underset{\operatorname{adj}_{u^{\prime}}}{\longrightarrow} f^{\prime !} u_{+}(\mathcal{E}) .
\end{array}
$$

est commutatif. On conclut en remarquant que son contour correspond au diagramme de (ii).

\subsection{Isomorphismes de recollement : cas rigide}

2.3.1. - Considérons les diagrammes commutatifs

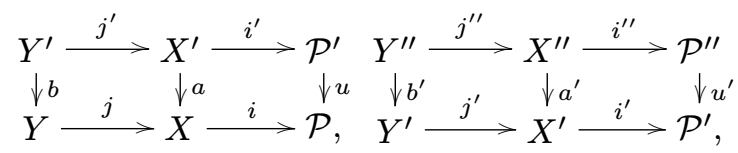

où $u$ (resp. $u^{\prime}$ ) est un morphisme de $\mathcal{V}$-schémas formels lisses, $j, j^{\prime}$ et $j^{\prime \prime}$ sont des immersions ouvertes de $k$-schémas, $i, i^{\prime}$ et $i^{\prime \prime}$ sont des immersions fermées. 
En notant $\phi=(u, a, b)$, on définit le foncteur image inverse extraordinaire par $\phi_{K}$ d'un $j^{\dagger} \mathcal{D}_{] X{ }_{\mathcal{P}}}$-module $E$ en posant $\phi_{K}^{!}(E):=j^{\prime \dagger} \mathcal{D}_{X^{\prime}{ }_{\mathcal{P}^{\prime}}} \otimes_{u_{K}^{-1} j^{\dagger} \mathcal{D}_{]_{X}{ }_{\mathcal{P}}}}^{\mathbb{L}}$ $u_{K}^{-1} E\left[d_{P^{\prime} / P}\right]$, où $d_{P^{\prime} / P}$ est la dimension relative de $P^{\prime}$ sur $P$. Si aucune confusion n'est à craindre, on écrira $u_{K}^{!}$au lieu de $\phi_{K}^{!}$. En désignant par $u_{K}^{*}$, l'image inverse usuelle (notations de $[3,2.2 .16]$ ), on obtient la relation : $u_{K}^{!}(E) \stackrel{\sim}{\longrightarrow}$ $u_{K}^{*}(E)\left[d_{P^{\prime} / P}\right]$.

D'après [3, 2.2.17.(i)], si $v: \mathcal{P}^{\prime} \rightarrow \mathcal{P}$ est un morphisme dont la restriction à $X^{\prime}$ se factorise par $a$, alors on dispose d'un isomorphisme $\epsilon_{u, v}: v_{K}^{*} \stackrel{\sim}{\longrightarrow}$ $u_{K}^{*}$ de foncteurs de la catégorie des $j^{\dagger} \mathcal{O}_{]_{X}{ }_{\mathcal{P}}}$-modules à connexion intégrable

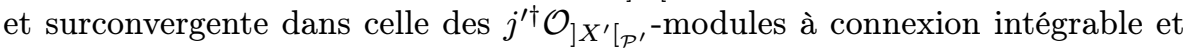
surconvergente, tel que $\epsilon_{u, u}=\mathrm{Id}$, et que, si $w: \mathcal{P}^{\prime} \rightarrow \mathcal{P}$ est un troisième morphisme coïncidant avec $u$ et $v$ sur $X^{\prime}$, on ait la condition de transitivité $\epsilon_{u, w}=\epsilon_{u, v} \circ \epsilon_{v, w}$. Si $v^{\prime}$ est un morphisme dont la restriction à $X^{\prime}$ se factorise par $a^{\prime}$, avec un raisonnement analogue à 2.1.11, on prouve les formules $\epsilon_{u \circ u^{\prime}, v \circ u^{\prime}}=$ $u_{K}^{\prime *} \circ \epsilon_{u, v}$ et $\epsilon_{u^{\prime}, v^{\prime}} \circ u_{K}^{*}=\epsilon_{u \circ u^{\prime}, u \circ v^{\prime}}$.

Dans le diagramme (de gauche par défaut) 2.3.1.1, supposons que l'on ne dispose que d'un morphisme $u_{0}: P^{\prime} \rightarrow P$ rendant commutatif le diagramme 2.3.1.1 où $u$ a été remplacé par $u_{0}$ (on suppose toujours que $P$ et $P^{\prime}$ se relèvent en des $\mathcal{V}$-schémas formels lisses $\mathcal{P}$ et $\left.\mathcal{P}^{\prime}\right)$. De manière analogue à [5, 2.1.6], on construit alors, par recollement, le foncteur $u_{0 K}^{*}$, de la catégorie des $j^{\dagger} \mathcal{O}_{X_{X}}$-modules à connexion intégrable et surconvergente dans celle des $j^{\prime \dagger} \mathcal{O}_{X^{\prime}}\left[_{\mathcal{P}^{\prime}}\right.$-modules à connexion intégrable et surconvergente. Précisons sa construction. Choisissons une application surjective $\rho: \Lambda^{\prime} \rightarrow \Lambda$, deux recouvrements ouverts affines $\left(\mathcal{P}_{\alpha}\right)_{\alpha \in \Lambda}$ de $\mathcal{P}$ et $\left(\mathcal{P}_{\alpha^{\prime}}^{\prime}\right)_{\alpha^{\prime} \in \Lambda^{\prime}}$ de $\mathcal{P}^{\prime}$ tels que $u_{0}$ se factorise par $P_{\alpha^{\prime}}^{\prime} \rightarrow P_{\rho\left(\alpha^{\prime}\right)}$. On note alors $X_{\alpha}:=X \cap P_{\alpha}$, $X_{\alpha \beta}:=X \cap P_{\alpha} \cap P_{\beta}$ et de même en rajoutant des primes. De plus, choisissons des relèvements $u_{\alpha^{\prime}}: \mathcal{P}_{\alpha^{\prime}}^{\prime} \rightarrow \mathcal{P}_{\rho\left(\alpha^{\prime}\right)}$ des factorisations induites par $u_{0}$. Si $E$ est un $j^{\dagger} \mathcal{O}_{X_{X}{ }_{\mathcal{P}}}$-module à connexion intégrable et surconvergente, on lui associe l'objet $\left(\left(\left.u_{\alpha^{\prime} K}^{*} E\right|_{] X_{\rho\left(\alpha^{\prime}\right)}\left[\mathcal{P}_{\rho\left(\alpha^{\prime}\right)}\right.}\right)_{\alpha^{\prime} \in \Lambda^{\prime}},\left(\eta_{\alpha^{\prime} \beta^{\prime}}^{\prime}\right)_{\alpha^{\prime}, \beta^{\prime} \in \Lambda^{\prime}}\right)$, où $\eta_{\alpha^{\prime} \beta^{\prime}}^{\prime}$ :

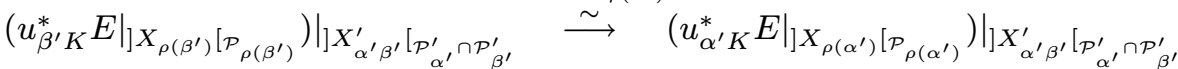
sont les isomorphismes de la forme $\epsilon$. Ils résultent de la condition de transitivité des isomorphismes de la forme $\epsilon$ ainsi que de leur comptabilité à l'image inverse que la famille $\left.\left(\left.u_{\alpha^{\prime} K}^{*} E\right|_{] X_{\rho\left(\alpha^{\prime}\right)}\left[\mathcal{P}_{\rho\left(\alpha^{\prime}\right)}\right.}\right)_{\alpha^{\prime} \in \Lambda^{\prime}},\left(\eta_{\alpha^{\prime} \beta^{\prime}}^{\prime}\right)_{\alpha^{\prime}, \beta^{\prime} \in \Lambda^{\prime}}\right)$ se recolle en un $j^{\prime \dagger} \mathcal{O}_{] X^{\prime}\left[\mathcal{P}^{\prime}\right.}$-module à connexion intégrable et surconvergente, celui-ci étant par définition $u_{0 K}^{*}(E)$. On vérifie ensuite que ce foncteur est indépendant des choix effectués.

Lorsque $u_{0}$ se relève en un morphisme $u: \mathcal{P}^{\prime} \rightarrow \mathcal{P}, u_{0 K}^{*}$ est canoniquement isomorphe à $u_{K}^{*}$. Enfin, si $u_{0}^{\prime}: P^{\prime \prime} \rightarrow P^{\prime}$ est un morphisme rendant commutatif le diagramme de droite de 2.3.1.1 lorsque $u^{\prime}$ a été remplacé par $u_{0}^{\prime}$, on dispose 
d'un isomorphisme canonique $\left(u_{0} \circ u_{0}^{\prime}\right)_{K}^{*} \stackrel{\sim}{\longrightarrow} u_{0 K}^{*} \circ u_{0 K}^{*}$, celui-ci étant transitif (pour la composition de diagrammes de la forme 2.3.1.1).

Soit $Y_{1}$ (resp. $Y_{1}^{\prime}$ ) un ouvert de $Y$ (resp. $Y^{\prime}$ ) tel que $b\left(Y_{1}^{\prime}\right) \subset Y_{1}$. En notant $j_{1}: Y_{1} \rightarrow X$ et $j_{1}^{\prime}: Y_{1}^{\prime} \rightarrow X^{\prime}$ les immersions ouvertes déduites, pour tout $j^{\dagger} \mathcal{O}_{] X[\mathcal{P}}$-module $E$ à connexion intégrable et surconvergente, on a le diagramme commutatif de gauche :

$$
\begin{array}{lll}
u_{K}^{*} j_{1}^{\dagger} E \longrightarrow j_{1}^{\prime \dagger} u_{K}^{*} E & u_{K}^{*}\left(E^{\vee}\right) \longrightarrow\left(u_{K}^{*}(E)\right)^{\vee} \\
\epsilon_{u, v} \uparrow & \epsilon_{u, v \uparrow} j_{1}^{\prime \dagger} \epsilon_{u, v} \uparrow & \epsilon_{u, v}^{\vee-1} \uparrow \\
v_{K}^{*} j_{1}^{\dagger} E_{1} \longrightarrow j_{1}^{\prime \dagger} v_{K}^{*} E, & v_{K}^{*}\left(E^{\vee}\right) \longrightarrow\left(v_{K}^{*}(E)\right)^{\vee} .
\end{array}
$$

De plus, on vérifie par construction celui de droite. En effet, l'isomorphisme $p_{2}^{*}\left(E_{1}^{\vee}\right) \stackrel{\sim}{\longrightarrow} p_{1}^{*}\left(E_{1}^{\vee}\right)$, modulo les isomorphismes (transitifs par rapport à la composée de deux morphismes) de commutation de l'image inverse au dual, est par définition l'inverse du dual de $p_{2}^{*}\left(E_{1}\right) \stackrel{\sim}{\longrightarrow} p_{1}^{*}\left(E_{1}\right)$.

2.4. Comparaison des isomorphismes formels et rigides de recollements. - Les deux propositions ci-après seront ensuite étendues, en 4.1 .2 et 4.1 .8 , au cas non relevable.

La première partie de la proposition qui suit est due à Noot-Huyghe ([29, 1.5.3]). Cependant, nous donnons ici une démonstration plus formelle qui fait intervenir la quasi-cohérence sur les schémas formels et nous nous assurons de sa transitivité.

Proposition 2.4.1. - Soient $f: \mathfrak{X}^{\prime} \rightarrow \mathfrak{X}$ un morphisme de $\mathcal{V}$-schémas formels lisses, $H$ un diviseur de $X, H^{\prime} \supset f_{0}^{-1}(H)$ un diviseur de $X^{\prime}, j: X \backslash H \hookrightarrow$

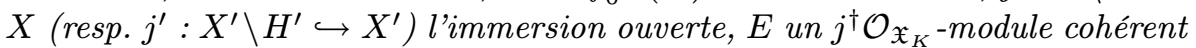
muni d'une connexion surconvergente $\nabla$ et $\mathcal{E}:=\operatorname{sp}_{*}(E)$, le $\mathcal{D}_{\mathfrak{X}, \mathbb{Q}}^{\dagger}\left({ }^{\dagger} H\right)$-module cohérent associé. On désignera par $f_{K}^{*}$ le foncteur image inverse de la catégorie des $j^{\dagger} \mathcal{O}_{\mathfrak{X}_{K}}$-modules cohérents à connexion surconvergente dans celle des $j^{\prime \dagger} \mathcal{O}_{\mathfrak{X}_{K}^{\prime}}$-modules cohérents à connexion surconvergente.

Il existe un isomorphisme $j^{\prime \dagger} \mathcal{D}_{\mathfrak{X}_{K}^{\prime}}$-linéaire, $f_{K}^{*} \operatorname{sp}^{*}(\mathcal{E}) \stackrel{\sim}{\longrightarrow} \operatorname{sp}^{*} f_{H^{\prime}, H}^{!}(\mathcal{E})\left[-d_{X^{\prime} / X}\right]$,

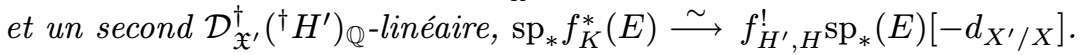

En outre, ces derniers sont transitifs, i.e., si $g: \mathfrak{X}^{\prime \prime} \rightarrow \mathfrak{X}^{\prime}$ est un second morphisme de $\mathcal{V}$-schémas formels lisses, si $H^{\prime \prime} \supset g_{0}^{-1}\left(H^{\prime}\right)$ est un diviseur de $X^{\prime \prime}$, alors le diagramme

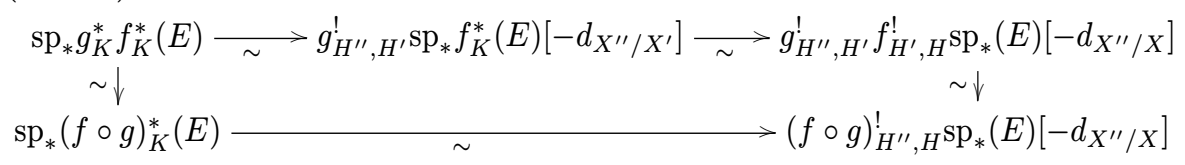


est commutatif. De même, on obtient un second diagramme commutatif en remplaçant dans 2.4.1.1 $\mathrm{sp}_{*}$ par $\mathrm{sp}^{*}, E$ par $\mathcal{E},-$ par + , en inversant le sens des flèches et en intervertissant les symboles en haut $*$ et !.

Démonstration. - Nous noterons abusivement $f^{!}, g^{!}$et $(f \circ g)$ à la place de $f_{H^{\prime}, H}^{!}, g_{H^{\prime \prime}, H^{\prime}}^{!}$et $(f \circ g)_{H^{\prime \prime}, H}^{!}$. On pose $\left.f^{*}(\mathcal{E}):=\mathcal{O}_{\mathfrak{X}^{\prime}}\left({ }^{\dagger} H^{\prime}\right)_{\mathbb{Q}} \otimes_{f^{-1} \mathcal{O}_{\mathfrak{X}}}{ }^{\dagger} H\right)_{\mathbb{Q}} f^{-1} \mathcal{E}$. Le foncteur $E \mapsto f_{K}^{*}(E) \stackrel{\sim}{\longrightarrow} j^{\dagger} \mathcal{O}_{\mathfrak{X}_{K}^{\prime}} \otimes_{f_{K}^{-1} j^{\dagger} \mathcal{O}_{\mathfrak{X}_{K}}} f_{K}^{-1}(E)$ est une image inverse de sites annelés, de même pour $f^{*}$ et $\mathrm{sp}^{*}$. Grâce à la transitivité de l'isomorphisme de commutation à la composition des images inverses de sites annelés, on obtient l'isomorphisme $f_{K}^{*} \mathrm{sp}^{*}(\mathcal{E}) \stackrel{\sim}{\longrightarrow} \mathrm{sp}^{*} f^{*}(\mathcal{E})$ commutant aux stratifications respectives (induites par celle de $\mathcal{E}$ qui provient de sa structure

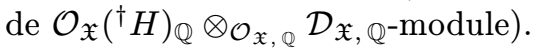

Soit $\mathcal{E}^{(\bullet)} \in L D_{\mathbb{Q}, \text { coh }}^{\mathrm{b}}\left(\widehat{\mathcal{D}}_{\mathcal{P}}^{(\bullet)}(T)\right)$ tel que $\lim _{\longrightarrow}\left(\mathcal{E}^{(\bullet)}\right) \stackrel{\sim}{\longrightarrow} \mathcal{E}$. On dispose, de manière analogue à $[6,4.3 .2]$, de l'isomorphisme canonique :

$$
f^{!}(\mathcal{E}) \stackrel{\sim}{\longrightarrow} \underset{\lim }{\longrightarrow}\left(\left(\widehat{\mathcal{B}}_{\mathfrak{X}^{\prime}}^{(m)}\left(H^{\prime}\right) \widehat{\otimes}_{f^{-1} \widehat{\mathcal{B}}_{\mathfrak{X}}^{(m)}(H)}^{\mathbb{L}} f^{-1} \mathcal{E}^{(m)}\right)_{m \in \mathbb{N}}\right)\left[d_{X^{\prime} / X}\right]
$$

De plus, puisque que $\mathcal{E}$ est un $\mathcal{O}_{\mathfrak{X}}\left({ }^{\dagger} H\right)_{\mathbb{Q}^{-}}$module cohérent, grâce au lemme [20, I.7.1] sur les foncteurs way-out, le morphisme canonique $\mathcal{O}_{\mathfrak{X}^{\prime}}\left({ }^{\dagger} H^{\prime}\right)_{\mathbb{Q}} \otimes_{\mathcal{O}_{\mathfrak{X}^{\prime}, \mathbb{Q}}}$ $\mathcal{D}_{\mathfrak{X}^{\prime}, \mathbb{Q}^{-}}$-linéaire

$$
\mathcal{O}_{\mathfrak{X}^{\prime}}\left({ }^{\dagger} H^{\prime}\right)_{\mathbb{Q}} \otimes_{f^{-1} \mathcal{O}_{\mathfrak{X}}\left({ }^{\dagger} H\right)_{\mathbb{Q}}} f^{-1} \mathcal{E} \rightarrow \underline{\lim }\left(\left(\widehat{\mathcal{B}}_{\mathfrak{X}^{\prime}}^{(m)}\left(H^{\prime}\right) \widehat{\otimes}_{f^{-1} \widehat{\mathcal{B}}_{\mathfrak{X}}^{(m)}(H)}^{\mathbb{L}} f^{-1} \mathcal{E}^{(m)}\right)_{m \in \mathbb{N}}\right)
$$

est un isomorphisme. Il en résulte un isomorphisme $f_{K}^{*} \operatorname{sp}^{*}(\mathcal{E}) \stackrel{\sim}{\longrightarrow} \operatorname{sp}^{*} f^{!}(\mathcal{E})\left[-d_{X^{\prime} / X}\right]$. En outre, la transitivité de cet isomorphisme est immédiate. En effet, il suffit d'invoquer la transitivité de l'isomorphisme de commutation à la composition des images inverses de sites annelés (et d'ajouter les isomorphismes de la forme $\left.f^{*}(\mathcal{E}) \stackrel{\sim}{\longrightarrow} f^{!}(\mathcal{E})\left[-d_{X^{\prime} / X}\right]\right)$.

Prouvons à présent le deuxième isomorphisme. D'après [4, 4.4.2], les foncteurs $\mathrm{sp}_{*}$ et $\mathrm{sp}^{*}$ sont des équivalences quasi-inverses de la catégorie des $j^{\dagger} \mathcal{O}_{\mathfrak{X}_{K}}$ modules cohérents munis d'une connexion intégrable dans celle des $\mathcal{O}_{\mathfrak{X}}\left({ }^{\dagger} H\right)_{\mathbb{Q}^{-}}$ modules cohérents munis d'une connexion intégrable (de même en rajoutant des primes). Il existe donc un unique isomorphisme $\mathcal{O}_{\mathfrak{X}^{\prime}}\left({ }^{\dagger} H^{\prime}\right)_{\mathbb{Q}} \otimes_{\mathcal{O}_{\mathfrak{X}^{\prime}, \mathbb{Q}}} \mathcal{D}_{\mathfrak{X}^{\prime}, \mathbb{Q}^{-}}$ linéaire, $\operatorname{sp}_{*} f_{K}^{*}(E) \stackrel{\sim}{\longrightarrow} f^{*} \operatorname{sp}_{*}(E)$, s'inscrivant dans le diagramme canonique

$$
\begin{aligned}
\operatorname{sp}^{*} f^{*} \operatorname{sp}_{*}(E) \longleftarrow f_{K}^{*} \operatorname{sp}^{*} \operatorname{sp}_{*}(E) \longrightarrow f_{K}^{*}(E) & \sim \\
\operatorname{sp}^{*} \operatorname{sp}_{*} f_{K}^{*}(E) & \sim f_{K}^{*}(E) .
\end{aligned}
$$

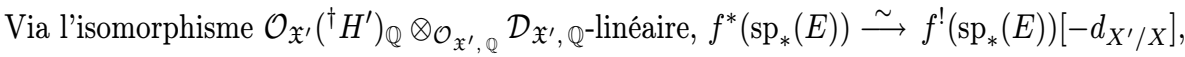
on construit le suivant $\operatorname{sp}_{*} f_{K}^{*}(E) \stackrel{\sim}{\longrightarrow} f^{!}\left(\operatorname{sp}_{*}(E)\right)\left[-d_{X^{\prime} / X}\right]$. Ce dernier, étant 
un morphisme $\mathcal{O}_{\mathfrak{X}^{\prime}}\left({ }^{\dagger} H^{\prime}\right)_{\mathbb{Q}} \otimes \mathcal{O}_{\mathfrak{X}^{\prime}, \mathbb{Q}} \mathcal{D}_{\mathfrak{X}^{\prime}, \mathbb{Q}}$-linéaire entre deux isocristaux surcon-

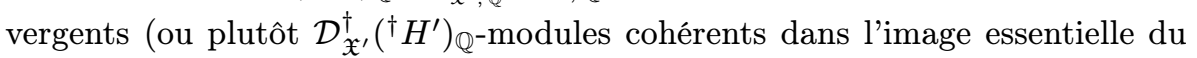
foncteur $\operatorname{sp}_{*}$ de $\left.[4,4.4 .5]\right)$, est $\mathcal{D}_{\mathfrak{X}^{\prime}}^{\dagger}\left({ }^{\dagger} H^{\prime}\right)_{\mathbb{Q}^{-}}$-linéaire.

Vérifions maintenant sa transitivité. Le diagramme 2.4.1.1 est commutatif, si et seulement si le suivant (qui correspond globalement à l'image de 2.4.1.1 par le foncteur sp*) l'est.

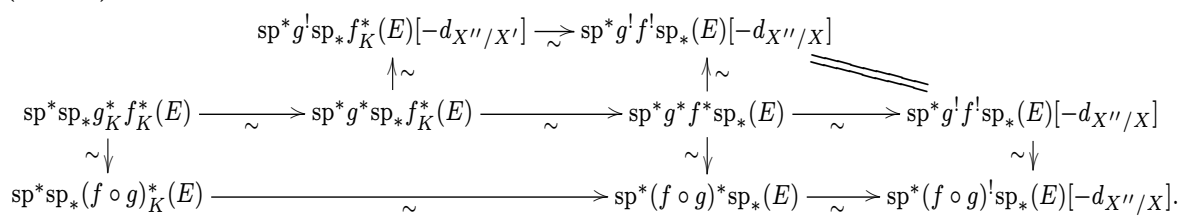

La commutativité des deux carrés et du triangle est aisée. Il reste à démontrer celle du rectangle en bas à gauche de 2.4.1.3. Or, celui-ci s'inscrit (à gauche) dans le suivant :

(2.4.1.4)

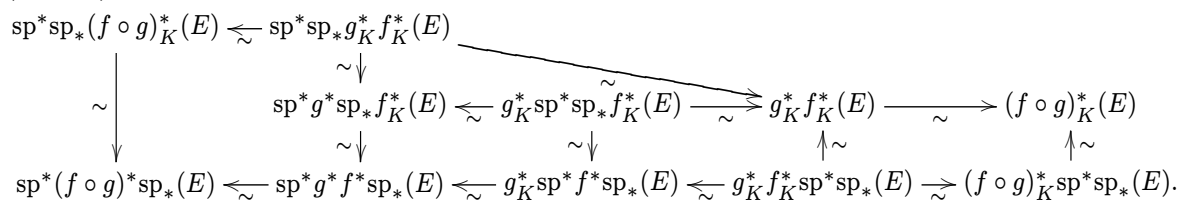

Le deuxième carré de droite et le triangle de 2.4.1.4 sont commutatifs par construction (voir 2.4.1.2), tandis que les deux autres carrés sont fonctoriels. Or, la flèche composée du bas de 2.4.1.4 est l'isomorphisme canonique $\mathrm{sp}^{*}(f \circ$ $g)^{*} \operatorname{sp}_{*}(E) \stackrel{\sim}{\longrightarrow}(f \circ g)_{K}^{*} \mathrm{sp}^{*} \operatorname{sp}_{*}(E)$. De plus, par fonctorialité de $\mathrm{sp}^{*} \mathrm{sp}_{*} \rightarrow \mathrm{Id}$, le morphisme composé du haut de 2.4.1.4 correspond au morphisme canonique $\mathrm{sp}^{*} \operatorname{sp}_{*}(f \circ g)_{K}^{*}(E) \stackrel{\sim}{\longrightarrow} \mathrm{sp}^{*}(f \circ g)^{*} \operatorname{sp}_{*}(E)$. Il en découle que le contour de 2.4.1.4 est le diagramme canonique ci-dessous

$$
\begin{aligned}
& \mathrm{sp}^{*} \operatorname{sp}_{*}(f \circ g)_{K}^{*}(E) \longrightarrow \underset{\sim \downarrow}{\sim} \longrightarrow \underset{\uparrow \sim}{\sim}(f \circ g)_{K}^{*}(E) \\
& \operatorname{sp}^{*}(f \circ g)^{*} \operatorname{sp}_{*}(E) \longleftarrow \sim(f \circ g)_{K}^{*} \operatorname{sp}^{*} \operatorname{sp}_{*}(E),
\end{aligned}
$$

qui est commutatif d'après 2.4.1.2. Le rectangle de gauche de 2.4.1.4 est donc aussi commutatif.

Remarques 2.4.2. - Avec les notations de 2.4.1, le diagramme canonique suivant

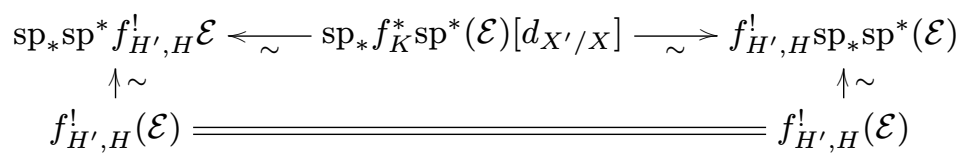

est commutatif. 
Démonstration. - Considérons le diagramme suivant

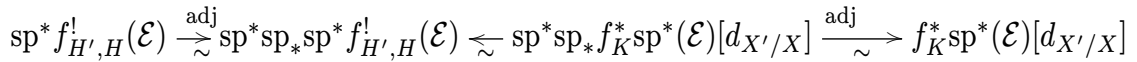

$$
\begin{aligned}
& \| \quad \sim \downarrow \quad \operatorname{adj} \uparrow \sim \\
& \mathrm{sp}^{*} f_{H^{\prime}, H}^{!}(\mathcal{E}) \underset{\text { adj }}{\sim} \mathrm{sp}^{*} f_{H^{\prime}, H}^{!} \operatorname{sp}_{*} \mathrm{sp}^{*}(\mathcal{E}) \underset{\sim}{\sim} f_{K}^{*} \operatorname{sp}^{*} \operatorname{sp}_{*} \mathrm{sp}^{*}(\mathcal{E})\left[d_{X^{\prime} / X}\right],
\end{aligned}
$$

où le rectangle à gauche est l'image par $\mathrm{sp}^{*}$ de celui de 2.4.2. Grâce à que 2.4.1.2, le carré est commutatif. De plus, on dispose du diagramme commutatif :

$$
\begin{aligned}
& \mathrm{sp}^{*} f_{H^{\prime}, H}^{!}(\mathcal{E}) \stackrel{\operatorname{adj}}{\sim} \mathrm{sp}^{*} \mathrm{sp}_{*} \mathrm{sp}^{*} f_{H^{\prime}, H}^{!}(\mathcal{E}) \longleftarrow \mathrm{sp}^{*} \operatorname{sp}_{*} f_{K^{*}}^{*} \mathrm{sp}^{*}(\mathcal{E})\left[d_{X^{\prime} / X}\right] \\
& \| \quad \sim \downarrow \text { adj } \quad \sim \downarrow \text { adj } \\
& \mathrm{sp}^{*} f_{H^{\prime}, H}^{!}(\mathcal{E})=\operatorname{sp}^{*} f_{H^{\prime}, H}^{!}(\mathcal{E}) \longleftarrow \sim f_{K}^{*} \mathrm{sp}^{*}(\mathcal{E})\left[d_{X^{\prime} / X}\right] \text {. }
\end{aligned}
$$

Il en résulte que l'isomorphisme composé du haut de 2.4.2.1, $\mathrm{sp}^{*} f_{H^{\prime}, H}^{!}(\mathcal{E}) \stackrel{\sim}{\leftarrow} f_{K}^{*} \mathrm{sp}^{*}(\mathcal{E})\left[d_{X^{\prime} / X}\right]$, est le morphisme canonique. De la même manière, en utilisant le diagramme commutatif suivant

$$
\begin{aligned}
& f_{K}^{*} \operatorname{sp}^{*}(\mathcal{E}) \stackrel{\operatorname{adj}}{\sim} f_{K}^{*} \operatorname{sp}^{*} \operatorname{sp}_{*} \operatorname{sp}^{*}(\mathcal{E}) \longrightarrow \operatorname{sp}^{*} f_{H^{\prime}, H^{\prime}}^{!} \operatorname{sp}_{*} \operatorname{sp}^{*}(\mathcal{E})\left[-d_{X^{\prime} / X}\right] \\
& \operatorname{adj} \uparrow \sim \quad \operatorname{adj} \uparrow \sim \\
& f_{K}^{*} \operatorname{sp}^{*}(\mathcal{E})=f_{K}^{*} \operatorname{sp}^{*}(\mathcal{E}) \longrightarrow \operatorname{sp}^{*} f_{H^{\prime}, H}^{!}(\mathcal{E})\left[-d_{X^{\prime} / X}\right] \text {, }
\end{aligned}
$$

on établit que le morphisme $f_{K}^{*} \mathrm{sp}^{*}(\mathcal{E})\left[d_{X^{\prime} / X}\right] \stackrel{\sim}{\longrightarrow} \mathrm{sp}^{*} f_{H^{\prime}, H}^{!}(\mathcal{E})$ de 2.4.2.1 passant par la droite puis en bas est le morphisme canonique de commutation (construit en 2.4.1). Le diagramme 2.4.2.1 est donc commutatif.

Proposition 2.4.3. - Soient $f: \mathfrak{X}^{\prime} \rightarrow \mathfrak{X}$ un morphisme de $\mathcal{V}$-schémas formels lisses, $H_{1} \subset H_{2}$ deux diviseurs de $X, H_{1}^{\prime} \subset H_{2}^{\prime}$ deux diviseurs de $X^{\prime}$ tels que, pour $i=1,2, H_{i}^{\prime} \supset f_{0}^{-1}\left(H_{i}\right)$. On note, pour $i=1,2, j_{i}: X \backslash H_{i} \hookrightarrow X$ et $j_{i}^{\prime}: X^{\prime} \backslash H_{i}^{\prime} \hookrightarrow X^{\prime}$ les immersions ouvertes. Pour tout isocristal $E_{1}$ sur $X \backslash H_{1}$ surconvergent le long de $H_{1}$, en notant et $\mathcal{E}_{1}=\mathrm{sp}_{*}\left(E_{1}\right)$, on dispose des isomorphismes $j_{2}^{\dagger} \mathrm{sp}^{*}\left(\mathcal{E}_{1}\right) \stackrel{\sim}{\longrightarrow} \mathrm{sp}^{*}\left({ }^{\dagger} H_{2}\right)\left(\mathcal{E}_{1}\right)$ et $\mathrm{sp}_{*} j_{2}^{\dagger}\left(E_{1}\right) \stackrel{\sim}{\longrightarrow}\left({ }^{\dagger} H_{2}^{\prime}\right) \circ \mathrm{sp}_{*}\left(E_{1}\right)$. Ceux-ci commutent aux images inverses extraordinaires, i.e., le diagramme canonique

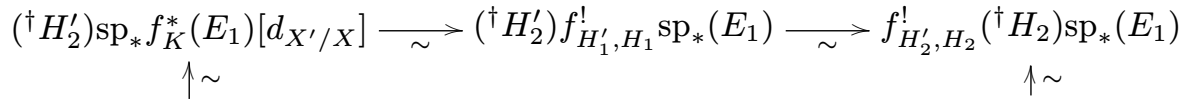

$$
\begin{aligned}
& \operatorname{sp}_{*} j_{2}^{\prime \dagger} f_{K}^{*}\left(E_{1}\right)\left[d_{X^{\prime} / X}\right] \longrightarrow \operatorname{sp}_{*} f_{K}^{*} j_{2}^{\dagger}\left(E_{1}\right)\left[d_{X^{\prime} / X}\right] \longrightarrow f_{H_{2}^{\prime}, H_{2}}^{!} \operatorname{sp}_{*} j_{2}^{\dagger}\left(E_{1}\right),
\end{aligned}
$$

est commutatif.

Démonstration. - Cela découle de la transitivité de 2.4.1. En effet, les foncteurs de la forme $j^{\dagger}$ (resp. $\left({ }^{\dagger} T\right)$ ) sont aussi des images inverses (resp. des images inverses extraordinaires). 
Afin de prouver que les isomorphismes de la forme $\epsilon$ et $\tau$ se correspondent via les foncteurs quasi-inverses $\mathrm{sp}_{*}$ et $\mathrm{sp}^{*}$ (voir 2.4.6), nous aurons besoin de quelques compléments à propos de la notion « topologiquement nilpotent ».

Lemme 2.4.4. - Soient $f: X \rightarrow S$ un morphisme lisse, $\mathcal{B}$ une $\mathcal{O}_{X}$-algèbre munie d'une structure compatible de $\mathcal{D}_{X / S}^{(m)}$-module et $\mathcal{E}$ un $\mathcal{B} \otimes \mathcal{O}_{X} \mathcal{D}_{X / S}^{(m)}$-module. On suppose que $p$ est localement nilpotent sur $S$, et qu'il existe sur $X$ un système de coordonnées locales $t_{1}, \ldots, t_{d}$. Les conditions suivantes sont équivalentes :

(i) Pour toute section e de $\mathcal{E}$, il existe localement un entier $N$ tel que, pour tout $\underline{k} \in \mathbb{N}^{d}$ tel que $|\underline{k}| \geq N, \underline{\partial}^{<\underline{k}>} e=0$.

(ii) La condition (i) est satisfaite pour tout système de coordonnées locales sur un ouvert de $X$.

(iii) Il existe un isomorphisme de $\mathcal{B} \otimes_{\mathcal{O}_{X}} \mathcal{P}_{X / S,(m)}$-algèbres

$$
\epsilon:\left(\mathcal{B} \otimes_{\mathcal{O}_{X}} \mathcal{P}_{X / S,(m)}\right) \otimes_{\mathcal{B}} \mathcal{E} \stackrel{\sim}{\longrightarrow} \mathcal{E} \otimes_{\mathcal{B}}\left(\mathcal{B} \otimes_{\mathcal{O}_{X}} \mathcal{P}_{X / S,(m)}\right)
$$

vérifiant la condition de cocycle, et induisant par réduction sur les $\mathcal{B} \otimes_{\mathcal{O}_{X}}$ $\mathcal{P}_{X / S,(m)}^{n}$ la $m$-PD-stratification de $\mathcal{E}$ relative à $\mathcal{B}$ (voir $\left.[10,1.1 .15]\right)$. De plus, cet isomorphisme est alors unique et déterminé, pour toute section locale e de $\mathcal{E}$, par la relation $\epsilon((1 \otimes 1) \otimes e)=\sum_{\underline{k} \geq 0} \underline{\widetilde{\partial}}^{<\underline{k}>} e \otimes \underline{\tau}^{\{\underline{k}\}}$, ò̀ $\underline{\widetilde{\tau}}^{\{\underline{k}\}}=1 \otimes \underline{\tau}^{\{\underline{k}\}}$ et $\underline{\tilde{\partial}}^{<\underline{k}>}:=1 \otimes \underline{\partial}^{<\underline{k}>}$.

Démonstration. - On calque [4, 2.3.7].

2.4.5. - On étend, de manière analogue à la suite de [4, 2.3.7], les notions de quasi-nilpotence, nilpotence et topologiquement nilpotence. Soient $\mathfrak{X}$ un $\mathcal{V}$-schéma formel, $H$ un diviseur de $X, n_{m} \geq m$ deux entiers et $\mathcal{E}$ un $\widehat{\mathcal{B}}_{\mathfrak{X}}^{\left(n_{m}\right)}(H) \widehat{\otimes}_{\mathcal{O}_{\mathfrak{X}}} \widehat{\mathcal{D}}_{\mathfrak{X}}^{(m)}$-module qui soit $\widehat{\mathcal{B}}_{\mathfrak{X}}^{\left(n_{m}\right)}(H)$-cohérent. Notons $X_{i}$ la réduction modulo $\pi^{i+1}$ de $\mathfrak{X}$ et $\mathcal{E}_{i}:=\mathcal{O}_{X_{i}} \otimes_{\mathcal{O}_{\mathfrak{X}}} \mathcal{E}$. On déduit par complétion de 2.4 .4 que les affirmations suivantes sont équivalentes :

(i) Le faisceau $\mathcal{E}$ est topologiquement nilpotent;

(ii) Il existe un isomorphisme de $\widehat{\mathcal{B}}_{\mathfrak{X}}^{\left(n_{m}\right)}(H) \widehat{\otimes}_{\mathcal{O}_{\mathfrak{X}}} \mathcal{P}_{X,(m)}$-algèbres

$$
\widehat{\epsilon}:\left(\widehat{\mathcal{B}}_{\mathfrak{X}}^{\left(n_{m}\right)}(H) \widehat{\otimes}_{\mathcal{O}_{\mathfrak{X}}} \mathcal{P}_{\mathfrak{X},(m)}\right) \otimes_{\widehat{\mathcal{B}}_{\mathfrak{X}}^{\left(n_{m}\right)}(H)} \mathcal{E} \stackrel{\sim}{\longrightarrow} \mathcal{E} \otimes_{\widehat{\mathcal{B}}_{\mathfrak{X}}^{\left(n_{m}\right)}(H)}\left(\widehat{\mathcal{B}}_{\mathfrak{X}}^{\left(n_{m}\right)}(H) \widehat{\otimes}_{\mathcal{O}_{\mathfrak{X}}} \mathcal{P}_{\mathfrak{X},(m)}\right)
$$

vérifiant la condition de cocycle, et induisant par réduction sur les $\mathcal{B}_{X_{i}}^{\left(n_{m}\right)}(H) \otimes_{\mathcal{O}_{X_{i}}}$ $\mathcal{P}_{X_{i},(m)}^{n}$ (pour tous entiers $i$ et $n$ ) la $m$-PD-stratification de $\mathcal{E}_{i}$ relative à $\mathcal{B}_{X_{i}}^{\left(n_{m}\right)}(H)$. De plus, cet isomorphisme est alors unique et satisfait, au dessus d'un ouvert possédant des coordonnées locales $t_{1}, \ldots, t_{d}$ et pour toute section locale $e$ de $\mathcal{E}$, la relation $\widehat{\epsilon}((1 \otimes 1) \otimes e)=\sum_{\underline{k} \geq 0} \underline{\widetilde{\partial}}^{<\underline{k}>} e \otimes \underline{\widetilde{\tau}}^{\{\underline{k}\}}$. Cette relation caractérise $\widehat{\epsilon}$. 
ThÉORÈme 2.4.6. - Avec les notations de 2.4.1, pour tout morphisme $f^{\prime}$ : $\mathfrak{X}^{\prime} \rightarrow \mathfrak{X}$ tel que $f_{0}^{\prime}=f_{0}$, les diagrammes suivants

$$
\begin{array}{ccc}
\operatorname{sp}_{*} f_{K}^{\prime *}(E)\left[d_{X^{\prime} / X}\right] \stackrel{\mathrm{sp}_{*}\left(\epsilon_{f, f^{\prime}}\right)}{\sim} \operatorname{sp}_{*} f_{K}^{*}(E)\left[d_{X^{\prime} / X}\right] & f_{K}^{\prime *} \operatorname{sp}^{*}(\mathcal{E})\left[d_{X^{\prime} / X}\right] \stackrel{\epsilon_{f, f^{\prime}}}{\sim} f_{K}^{*} \operatorname{Sp}^{*}(\mathcal{E})\left[d_{X^{\prime} / X}\right] \\
\sim \downarrow & \sim \downarrow & \sim \downarrow \\
f_{H^{\prime}, H}^{\prime \prime} \operatorname{sp}_{*}(E) \stackrel{\tau_{f, f^{\prime}, H^{\prime}, H}}{\sim} f_{H^{\prime}, H^{\prime}}^{!} \operatorname{sp}_{*}(E), & \operatorname{sp}^{*} f_{H^{\prime}, H}^{\prime !}(\mathcal{E}) \stackrel{\mathrm{sp}^{*}\left(\tau_{f, f^{\prime}, H^{\prime}, H}\right)}{\sim} f_{H^{\prime}, H^{\prime}}^{!} \operatorname{sp}_{*}(\mathcal{E}),
\end{array}
$$

où les isomorphismes verticaux résultent de 2.4 .1 et ceux horizontaux sont construits dans les sections 2.1.10 et de 2.3.1, sont commutatifs.

Démonstration. - L'assertion étant locale en $\mathfrak{X}$ et $\mathfrak{X}^{\prime}$, on se ramène au cas où $\mathfrak{X}=\operatorname{Spf} A$ et $\mathfrak{X}^{\prime}=\operatorname{Spf} A^{\prime}$ sont affines et possèdent des coordonnées locales et où $H$ (resp. $H^{\prime}$ ) est l'ensemble des zéros modulo $\mathfrak{m}$ d'un élément $g$ de $A$ (resp. $g^{\prime}$ de $\left.A^{\prime}\right)$. Nous prenons les notations de [3, 1.1.8 et 1.2]. Pour tout $1>\lambda \geq|\pi|$, on a ainsi $\left.U_{\lambda}:=\right] X[\mathfrak{X}-] H\left[\mathfrak{x} \lambda=\left\{x \in \mathfrak{X}_{K} ;|g(x)| \geq \lambda\right\}, U_{\lambda}^{\prime}:=\right] X^{\prime}\left[\mathfrak{X}^{\prime}-\right] H^{\prime}\left[\mathfrak{X}^{\prime} \lambda=\left\{x^{\prime} \in\right.\right.$ $\left.\mathfrak{X}_{K}^{\prime} ;\left|g^{\prime}\left(x^{\prime}\right)\right| \geq \lambda\right\}$ (l'hypothèse $\lambda \geq|\pi|$ implique que ceux-ci ne dépendent pas du choix des sections $g$ et $g^{\prime}$ définissant $H$ et $\left.H^{\prime}\right)$. Pour tout $m \in \mathbb{N}$, on écrira $\lambda_{m}$ ou $\mu_{m}$ pour $p^{-1 / p^{m+1}}$. On remarque que $\Gamma\left(\mathfrak{X}, \widehat{\mathcal{B}}_{\mathfrak{X}}^{(m)}(H)\right)_{\mathbb{Q}}=\Gamma\left(U_{\lambda_{m}}, \mathcal{O}_{\mathfrak{X}_{K}}\right)$.

Soit $m_{0}$ suffisamment grand tel qu'il existe un $\mathcal{O}_{\lambda_{m_{0}}}$-module cohérent $E_{0}$, muni d'une connexion intégrable $\nabla_{0}$, et un isomorphisme $(E, \nabla) \stackrel{\sim}{\longrightarrow}$ $j^{\dagger}\left(E_{0}, \nabla_{0}\right)$. Pour tout $m \geq m_{0}$, on note $\left(E_{m}, \nabla_{m}\right)$ la restriction de $\left(E_{0}, \nabla_{0}\right)$ à $U_{\lambda_{m}}, \mathcal{E}_{0}=\operatorname{sp}_{*}\left(E_{0}\right)$ et $\mathcal{E}^{(m)}=\operatorname{sp}_{*}\left(E_{m}\right)$, où sp est le composé $U_{\lambda_{m}} \subset \mathfrak{X}_{K} \stackrel{\text { sp }}{\longrightarrow} \mathfrak{X}$ induit par le morphisme de spécialisation.

Notons respectivement $p_{1}$ et $p_{2}$ les projections $] X\left[\mathfrak{X}^{2} \rightarrow \mathfrak{X}_{K}\right.$ à gauche et à droite, $\mathcal{I}$ l'idéal définissant l'immersion diagonale $\mathfrak{X}_{K} \hookrightarrow \mathfrak{X}_{K}^{2}, \mathcal{P}^{n}=\mathcal{O}_{\mathfrak{X}_{K}^{2}} / \mathcal{I}^{n+1}$

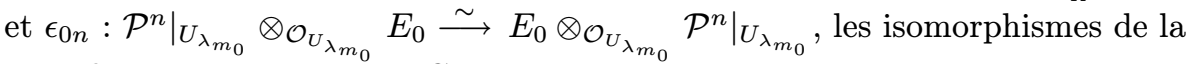
stratification associée à $\nabla_{0}$. Comme la connexion $\nabla$ est surconvergente, d'après $[3,2.2 .6]$, il existe un voisinage strict $V^{\prime}$ de $] X \backslash H\left[\mathfrak{X}^{2}\right.$ dans $] X\left[\mathfrak{X}^{2}\right.$, contenu dans $p_{1}^{-1}\left(U_{\lambda_{m_{0}}}\right) \cap p_{2}^{-1}\left(U_{\lambda_{m_{0}}}\right)$, tel que $\epsilon$ soit de la forme $\epsilon=j^{\dagger}\left(\epsilon_{0}\right)$, où $\epsilon_{0}$ : $\left.\left.p_{2}^{*}\left(E_{m_{0}}\right)\right|_{V^{\prime}} \stackrel{\sim}{\longrightarrow} p_{1}^{*}\left(E_{m_{0}}\right)\right|_{V^{\prime}}$ est un isomorphisme de $\mathcal{O}_{V^{\prime}}$-modules, induisant pour tout $n$, par réduction modulo $\mathcal{I}^{n+1}$, la restriction à $V^{\prime} \cap \mathfrak{X}_{K}$ des isomorphismes $\epsilon_{0, n}$.

Comme $f_{0}^{-1}(H) \subset H^{\prime}$, il existe des sections $a^{\prime}$ et $b^{\prime}$ de $A^{\prime}$ telles que $g^{\prime}=$ $a^{\prime} f^{*}(g)+\pi b^{\prime}$. Si $\lambda>|\pi|$, il en résulte, pour tout $x^{\prime} \in U_{\lambda}^{\prime}$, que $\lambda \leq\left|f_{K}^{*}(g)\left(x^{\prime}\right)\right|=$ $\left|g\left(f_{K}\left(x^{\prime}\right)\right)\right|$. Ainsi, lorsque $\lambda>|\pi|, f_{K}$ induit le morphisme $U_{\lambda}^{\prime} \rightarrow U_{\lambda}$. De même en remplaçant $f$ par $f^{\prime}$.

D'après [4, 4.4], il existe une suite d'entiers $\left(n_{m}\right)_{m \in \mathbb{N}}$ telle que $n_{m}>$ $\max \left\{m, m_{0}\right\}$ et telle que $\mathcal{E}^{\left(n_{m}\right)}$ soit muni d'une structure de $\widehat{\mathcal{B}}_{\mathfrak{X}}^{\left(n_{m}\right)}(H) \widehat{\otimes} \widehat{\mathcal{D}}_{\mathfrak{X}, \mathbb{Q}^{-}}^{(m)}$ module cohérent topologiquement nilpotent. Via [4, 4.4.7], il existe un 
$\widehat{\mathcal{B}}_{\mathfrak{X}}^{\left(n_{m}\right)}(H) \widehat{\otimes} \widehat{\mathcal{D}}_{\mathfrak{X}}^{(m)}$-module cohérent $\stackrel{\circ}{\mathcal{E}}^{\left(n_{m}\right)}$, cohérent sur $\widehat{\mathcal{B}}_{\mathfrak{X}}^{\left(n_{m}\right)}(H)$ et sans

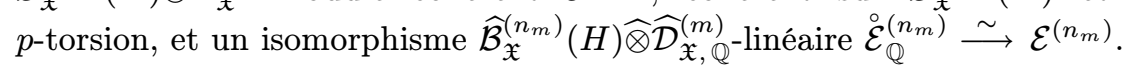

Comme $1>\lambda_{n_{m}}>\eta_{m}>|\pi|,[X]_{\mathfrak{X}^{2} \eta_{m}} \cap p_{1}^{-1}\left(U_{\lambda_{n_{m}}}\right)=[X]_{\mathfrak{X}^{2} \eta_{m}} \cap$ $p_{2}^{-1}\left(U_{\lambda_{n_{m}}}\right)=: V_{\eta_{m} \lambda_{n_{m}}}$. Notons $h=\left(f, f^{\prime}\right): \mathfrak{X}^{\prime} \rightarrow \mathfrak{X} \times_{\mathcal{S}} \mathfrak{X}$. Puisque $f$ et $f^{\prime}$ coïncident sur $X^{\prime}, h^{*}\left(\tau_{i}\right) \in \pi A^{\prime}$. On en déduit que pour tout $x^{\prime} \in \mathfrak{X}_{K}^{\prime}$, $h_{K}\left(x^{\prime}\right) \in[X]_{\mathfrak{X}^{2}|\pi|} \subset[X]_{\mathfrak{X}^{2} \eta_{m}}$. Il en découle que le morphisme $h_{K}$ induit la flèche $U_{\lambda_{n_{m}}}^{\prime} \rightarrow V_{\eta_{m} \lambda_{n_{m}}}$, notée abusivement $h_{k}$, qui s'insère dans le diagramme commutatif

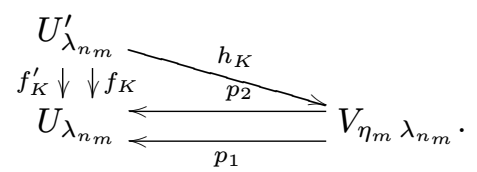

Via [3, 1.2.2], quitte à augmenter $n_{m}$, on peut supposer $V_{\eta_{m} \lambda_{n_{m}}} \subset V^{\prime}$. On note alors $\epsilon_{m}: p_{2}^{*}\left(E_{n_{m}}\right) \stackrel{\sim}{\longrightarrow} p_{1}^{*}\left(E_{n_{m}}\right)$ l'isomorphisme $\left.\epsilon_{0}\right|_{V_{\eta_{m} \lambda_{n_{m}}}}$. Pour tout $n$, la restriction à $U_{\lambda_{n_{m}}}$ des réduction modulo $\mathcal{I}^{n+1}$ des isomorphismes $\epsilon_{m}$ est $\left.\epsilon_{0 n}\right|_{U_{\lambda_{n_{m}}}}$ et correspond donc aux isomorphismes de la stratification de $E_{n_{m}}$ induite par $\nabla_{n_{m}}$.

En considérant $\mathfrak{X}^{2}$ comme $\mathfrak{X}$-schéma via la projection à gauche, notons $\phi$ : $\mathfrak{X}^{2} \rightarrow \mathfrak{X} \times \widehat{\mathbb{A}}_{\mathcal{V}}^{d}$ le morphisme de $\mathfrak{X}$-schémas formels défini par $\tau_{1}, \ldots, \tau_{d}$. Puisque $\mathfrak{X}$ possède des coordonnées locales, il résulte du théorème de fibration fort [3, 1.3.7] qu'il existe des voisinages stricts $V^{\prime}$ de $] X \backslash H\left[\mathfrak{X}^{2}\right.$ dans $] X\left[\mathfrak{X}_{\mathfrak{X}^{2}}\right.$ et $V^{\prime \prime}$ de ]$X \backslash H\left[_{\mathfrak{X} \times \widehat{\mathbb{A}}_{\mathcal{V}}^{d}}\right.$ dans $] X\left[_{\mathfrak{X} \times \widehat{\mathbb{A}}_{\mathcal{V}}^{d}}\right.$ tels que $\phi_{K}$ induise l'isomorphisme $V^{\prime} \stackrel{\sim}{\longrightarrow} V^{\prime \prime}$. Par construction de $\phi, \phi_{K}^{-1}\left(U_{\lambda_{n_{m}}} \times D\left(0, \eta_{m}{ }^{+}\right)\right)=V_{\eta_{m} \lambda_{n_{m}}}$. De plus, quitte à accroître $n_{m}$ et grâce à $[3,1.2 .2]$ (et à $[3,1.2 .3$.(iii)] pour la deuxième inclusion), on peut supposer $U_{\lambda_{n_{m}}} \times D\left(0, \eta_{m}{ }^{+}\right) \subset V^{\prime \prime}$ et $V_{\eta_{m} \lambda_{n_{m}}} \subset V^{\prime}$. L'isomorphisme $\phi_{K}: V^{\prime} \stackrel{\sim}{\longrightarrow} V^{\prime \prime}$ induit alors le suivant

$$
V_{\eta_{m} \lambda_{n_{m}}} \stackrel{\sim}{\longrightarrow} U_{\lambda_{n_{m}}} \times D\left(0, \eta_{m}^{+}\right) .
$$

Grâce à [3, 2.2.12], il en dérive que l'isomorphisme $\epsilon_{m}$ est déterminé, pour toute section $e$ de $E_{n_{m}}$, par la formule $\epsilon_{m}(1 \otimes e)=\sum_{\underline{k} \geq 0} \underline{\partial}^{[\underline{k}]} e \otimes \underline{\tau}^{\underline{k}}$. En passant à la limite sur $m$ l'isomorphisme $h_{K}^{*}\left(\epsilon_{m}\right): f_{K}^{\prime *}\left(E_{n_{m}}\right) \stackrel{\sim}{\longrightarrow} f_{K}^{*}\left(E_{n_{m}}\right)$ (ou en lui appliquant le foncteur $j^{\dagger}$ ), on obtient $\epsilon_{f, f^{\prime}}$.

Notons $\widehat{\mathcal{P}}_{\mathfrak{X},(m)}=\lim _{\longleftarrow} \mathcal{P}_{X_{i}(m)}, \widehat{P}_{\mathfrak{X},(m)}=\Gamma\left(\mathfrak{X}, \widehat{\mathcal{P}}_{\mathfrak{X},(m)}\right), \widehat{\mathcal{P}}_{\mathfrak{X},(m)}^{\prime}=$ $\lim _{i} \widehat{\mathcal{B}}_{X_{i}}^{\left(n_{m}\right)}(H) \otimes_{\mathcal{O}_{X_{i}}} \mathcal{P}_{X_{i}(m)}$ et $\widehat{P}_{\mathfrak{X},(m)}^{\prime}=\Gamma\left(\mathfrak{X}, \widehat{\mathcal{P}}_{\mathfrak{X},(m)}^{\prime}\right)$. Désignons par $\tilde{f}_{i}$ et $\tilde{f}_{i}^{\prime}$ les morphismes d'espaces annelés $\left(X_{i}^{\prime}, \mathcal{B}_{X_{i}^{\prime}}^{\left(n_{m}\right)}\left(H^{\prime}\right)\right) \rightarrow\left(X_{i}, \mathcal{B}_{X_{i}}^{\left(n_{m}\right)}(H)\right)$ induits par $f_{i}$ et $f_{i}^{\prime}$, et par $\delta_{\tilde{f}_{i}, \tilde{f}_{i}^{\prime}}:\left(X_{i}^{\prime}, \mathcal{B}_{X_{i}^{\prime}}^{\left(n_{m}\right)}\left(H^{\prime}\right)\right) \rightarrow \widetilde{\Delta}_{X_{i}(m)}$ le morphisme déduit (notations de 2.1.5). En passant à la limite inductive, on obtient le morphisme d'espaces annelés $\delta_{\tilde{f}, \tilde{f}^{\prime}}:\left(\mathfrak{X}^{\prime}, \widehat{\mathcal{B}}_{\mathfrak{X}^{\prime}}^{\left(n_{m}\right)}\left(H^{\prime}\right)\right) \rightarrow \widetilde{\Delta}_{\mathfrak{X}(m)}$ (où $\widetilde{\Delta}_{\mathfrak{X}(m)}$ 
désigne la limite inductive des $\widetilde{\Delta}_{X_{i}(m)}$, i.e., l'espace topologique est celui des $\widetilde{\Delta}_{X_{i}(m)}$ tandis que le faisceau d'anneaux correspond à la limite projective des faisceaux structuraux des $\left.\widetilde{\Delta}_{X_{i}(m)}\right)$ s'inscrivant dans le diagramme commutatif :

$$
\begin{aligned}
& \left(\mathfrak{X}^{\prime}, \widehat{\mathcal{B}}_{\mathfrak{X}^{\prime}}^{\left(n_{m}\right)}\left(H^{\prime}\right)\right) \\
& \tilde{f}^{\prime} \downarrow \downarrow \tilde{f} \\
& \left(\mathfrak{X}, \widehat{\mathcal{B}}_{\mathfrak{X}}^{\left(n_{m}\right)}(H)\right) \underset{\delta_{\tilde{f}, \tilde{f}^{\prime}}}{\stackrel{p_{2}}{\longleftarrow}} \widetilde{\Delta}_{\mathfrak{p _ { 1 }}(m)} .
\end{aligned}
$$

Il dérive de 2.4.5 que la structure de $\widehat{\mathcal{B}}_{\mathfrak{X}}^{\left(n_{m}\right)}(H) \widehat{\otimes} \widehat{\mathcal{D}}_{\mathfrak{X}}^{(m)}$-module topologiquement nilpotent de $\stackrel{\circ}{\mathcal{E}}^{\left(n_{m}\right)}$ induit un isomorphisme $\widehat{\epsilon}_{m}: \tilde{p}_{2}^{*}\left(\stackrel{\circ}{\mathcal{E}}^{\left(n_{m}\right)}\right) \stackrel{\sim}{\longrightarrow} \tilde{p}_{1}^{*}\left(\stackrel{\circ}{\mathcal{E}}^{\left(n_{m}\right)}\right)$. En lui appliquant $\delta_{\tilde{f}, \tilde{f}^{\prime}}^{*}$, il en découle le suivant $: \tilde{f}^{\prime *}\left(\mathcal{\mathcal { E }}^{\left(n_{m}\right)}\right) \stackrel{\sim}{\longrightarrow} \tilde{f}^{*}\left(\stackrel{\mathcal{E}}{ }^{\left(n_{m}\right)}\right)$. En tensorisant par $\mathbb{Q}$, puis en passant à la limite inductive sur $m$, on obtient (modulo le décalage $\left[d_{X^{\prime} / X}\right]$ ) l'isomorphisme $\tau_{\tilde{f}, \tilde{f}^{\prime}}$.

Or, on dispose d'un homomorphisme canonique d'anneaux $\Gamma\left([X]_{\mathfrak{X}^{2} \eta_{m}}, \mathcal{O}_{[X]_{\mathfrak{X}^{2} \eta_{m}}}\right) \rightarrow P_{\mathfrak{X},(m) \mathbb{Q}}$ (voir le début de la preuve de $\left.[1,3.1 .2]\right)$. Ce dernier est $A \otimes_{\mathcal{V}} K$-linéaire pour les structures droite et gauche et envoie $\tau_{i}$ $\operatorname{sur} \tau_{i}$. Il en résulte un morphisme $\Gamma\left(V_{\eta_{m} \lambda_{n_{m}}}, \mathcal{O}_{V_{\eta_{m} \lambda_{n_{m}}}}\right) \rightarrow P_{\mathfrak{X},(m) \mathbb{Q}}$. Via cette extension, les $\epsilon_{m}: p_{2}^{*}\left(E_{n_{m}}\right) \stackrel{\sim}{\longrightarrow} p_{1}^{*}\left(E_{n_{m}}\right)$ induisent alors (modulo les foncteurs quasi-inverses $\mathrm{sp}_{*}$ et $\left.\mathrm{sp}^{*}\right)$ les isomorphismes $\eta_{m}: p_{2}^{*}\left(\stackrel{\circ}{\mathcal{E}}^{\left(n_{m}\right)}\right)_{\mathbb{Q}} \stackrel{\sim}{\longrightarrow} p_{1}^{*}\left(\stackrel{\circ}{\mathcal{E}}^{\left(n_{m}\right)}\right)_{\mathbb{Q}}$. Puisque $\epsilon_{m}(1 \otimes e)=\sum_{\underline{k} \geq 0} \underline{\partial}^{[\underline{k}]} e \otimes \underline{\tau}^{\underline{k}}$ et que $\widehat{\epsilon}_{m \mathbb{Q}}$ est caractérisé par la relation $\widehat{\epsilon}_{m \mathbb{Q}}((1 \otimes 1) \otimes e)=\sum_{\underline{k} \geq 0} \underline{\widetilde{\partial}}^{<\underline{k}>} e \otimes \underline{\widetilde{\tau}}^{\{\underline{k}\}}$ (cela découle de 2.4.5), $\eta_{m}=\widehat{\epsilon}_{m \mathbb{Q}}$. Il en dérive que les deux isomorphismes $f_{K}^{\prime *}\left(E_{n_{m}}\right) \stackrel{\sim}{\longrightarrow} f_{K}^{*}\left(E_{n_{m}}\right)$ et $\tilde{f}^{\prime *}\left(\stackrel{\circ}{\mathcal{E}}^{\left(n_{m}\right)}\right)_{\mathbb{Q}} \stackrel{\sim}{\longrightarrow} \tilde{f}^{*}\left(\stackrel{\circ}{\mathcal{E}}^{\left(n_{m}\right)}\right)_{\mathbb{Q}}$ se correspondent (modulo les foncteurs quasiinverses $\mathrm{sp}_{*}$ et $\left.\mathrm{sp}^{*}\right)$. On conclut en passant à la limite sur $m$.

2.5. Construction de $\mathrm{sp}_{+} \cdot$ - D'après une remarque de Berthelot, la procédure de recollement de la section [8,2] est incorrecte. Avec les notations de [8, 2], cela vient du fait que les schémas formels de la forme $\mathfrak{X}_{\alpha} \times_{\mathcal{P}} \mathfrak{X}_{\beta}$ ne sont pas plats en général. Nous donnerons ici une procédure améliorée et nous vérifierons par la suite avec celle-ci (voir 4) les résultats de la section [8, 2].

2.5.1. - Nous garderons, sauf mention explicite du contraire, les suivantes : on se donne $\mathcal{P}$ un $\mathcal{V}$-schéma formel séparé et lisse, $X$ un sous-schéma fermé $k$-lisse de $P$ et $T$ un diviseur de $P$ tel que $T_{X}:=T \cap X$ soit un diviseur de $X$. On note $\mathfrak{U}:=\mathcal{P} \backslash T, Y:=X \backslash T_{X}$ et $j: Y \hookrightarrow X$ l'immersion ouverte. En notant $\operatorname{Isoc}^{\dagger}(Y, X / K)$ la catégorie des isocristaux sur $Y$ surconvergent le long de $T \cap X$ et $\operatorname{Coh}(X, \mathcal{P}, T)$ celle des $\mathcal{D}_{\mathcal{P}}^{\dagger}\left({ }^{\dagger} T\right)_{\mathbb{Q}^{-} \text {-modules cohérents à }}$ support dans $X$, nous allons maintenant construire le foncteur $\operatorname{sp}_{X \hookrightarrow \mathcal{P}, T,+}$ : $\operatorname{Isoc}^{\dagger}(Y, X / K) \rightarrow \operatorname{Coh}(X, \mathcal{P}, T)$. Pour cela, il ne coûte rien de supposer $X$ intègre. En effet, $X$ est la somme directe de ses composantes irréductibles notées 
$X_{r}$. On bénéficie des équivalences canoniques de catégories : $\operatorname{Isoc}^{\dagger}(Y, X / K) \cong$ $\prod_{r} \operatorname{Isoc}^{\dagger}\left(Y \cap X_{r}, X_{r} / K\right)$ et $\operatorname{Coh}(X, \mathcal{P}, T) \cong \prod_{r} \operatorname{Coh}\left(X_{r}, \mathcal{P}, T\right)$. Si $E$ est un objet de $\operatorname{Isoc}^{\dagger}(Y, X / K)$ et $E_{r}$ sont les objets induits sur Isoc ${ }^{\dagger}\left(Y \cap X_{r}, X_{r} / K\right)$, il suffit alors de poser $\operatorname{sp}_{X \hookrightarrow \mathcal{P}, T,+}(E):=\bigoplus_{r} \operatorname{sp}_{X_{r} \hookrightarrow \mathcal{P}, T,+}\left(E_{r}\right)$. On remarque enfin que dans le cas où $X$ est intègre, ce que l'on supposera dans la suite de cette section, soit $T \supset X$ (les catégories $\operatorname{Isoc}^{\dagger}(Y, X / K)$ et $\operatorname{Coh}(X, \mathcal{P}, T)$ sont alors nulles) ou soit $T \cap X$ est un diviseur de $X$. Cette dernière hypothèse est donc superflue mais nous la conserverons afin d'éviter de jongler avec les cas.

On fixe $\left(\mathcal{P}_{\alpha}\right)_{\alpha \in \Lambda}$ un recouvrement d'ouverts de $\mathcal{P}$. On note $\mathcal{P}_{\alpha \beta}:=\mathcal{P}_{\alpha} \cap \mathcal{P}_{\beta}$, $\mathcal{P}_{\alpha \beta \gamma}:=\mathcal{P}_{\alpha} \cap \mathcal{P}_{\beta} \cap \mathcal{P}_{\gamma}, X_{\alpha}:=X \cap P_{\alpha}, X_{\alpha \beta}:=X_{\alpha} \cap X_{\beta}$ et $X_{\alpha \beta \gamma}:=X_{\alpha} \cap X_{\beta} \cap X_{\gamma}$. De plus, on notera $Y_{\alpha}$ l'ouvert de $X_{\alpha}$ complémentaire de $T, Y_{\alpha \beta}:=Y_{\alpha} \cap Y_{\beta}$, $Y_{\alpha \beta \gamma}:=Y_{\alpha} \cap Y_{\beta} \cap Y_{\gamma}, j_{\alpha}: Y_{\alpha} \hookrightarrow X_{\alpha}, j_{\alpha \beta}: Y_{\alpha \beta} \hookrightarrow X_{\alpha \beta}$ et $j_{\alpha \beta \gamma}: Y_{\alpha \beta \gamma} \hookrightarrow X_{\alpha \beta \gamma}$ les immersions ouvertes canoniques. On suppose de plus que pour tout $\alpha \in \Lambda$, $X_{\alpha}$ est affine (par exemple lorsque le recouvrement $\left(\mathcal{P}_{\alpha}\right)_{\alpha \in \Lambda}$ est affine). Comme $P$ est séparé, pour tous $\alpha, \beta, \gamma \in \Lambda, X_{\alpha \beta}$ et $X_{\alpha \beta \gamma}$ sont donc affines.

Pour tout triplet $(\alpha, \beta, \gamma) \in \Lambda^{3}$, choisissons $\mathfrak{X}_{\alpha}$ (resp. $\left.\mathfrak{X}_{\alpha \beta}, \mathfrak{X}_{\alpha \beta \gamma}\right)$ des $\mathcal{V}$ schémas formels lisses relevant $X_{\alpha}$ (resp. $X_{\alpha \beta}, X_{\alpha \beta \gamma}$ ), $p_{1}^{\alpha \beta}: \mathfrak{X}_{\alpha \beta} \rightarrow \mathfrak{X}_{\alpha}$ (resp. $p_{2}^{\alpha \beta}: \mathfrak{X}_{\alpha \beta} \rightarrow \mathfrak{X}_{\beta}$ ) des relèvements de $X_{\alpha \beta} \rightarrow X_{\alpha}$ (resp. $X_{\alpha \beta} \rightarrow X_{\beta}$ ). Rappelons que grâce à Elkik ([18] de tels relèvements existent bien.

De même, pour tout triplet $(\alpha, \beta, \gamma) \in \Lambda^{3}$, on choisit des relèvements $p_{12}^{\alpha \beta \gamma}$ : $\mathfrak{X}_{\alpha \beta \gamma} \rightarrow \mathfrak{X}_{\alpha \beta}, p_{23}^{\alpha \beta \gamma}: \mathfrak{X}_{\alpha \beta \gamma} \rightarrow \mathfrak{X}_{\beta \gamma}, p_{13}^{\alpha \beta \gamma}: \mathfrak{X}_{\alpha \beta \gamma} \rightarrow \mathfrak{X}_{\alpha \gamma}, p_{1}^{\alpha \beta \gamma}: \mathfrak{X}_{\alpha \beta \gamma} \rightarrow \mathfrak{X}_{\alpha}$ $p_{2}^{\alpha \beta \gamma}: \mathfrak{X}_{\alpha \beta \gamma} \rightarrow \mathfrak{X}_{\beta}, p_{3}^{\alpha \beta \gamma}: \mathfrak{X}_{\alpha \beta \gamma} \rightarrow \mathfrak{X}_{\gamma}, u_{\alpha}: \mathfrak{X}_{\alpha} \hookrightarrow \mathcal{P}_{\alpha}, u_{\alpha \beta}: \mathfrak{X}_{\alpha \beta} \hookrightarrow \mathcal{P}_{\alpha \beta}$ et $u_{\alpha \beta \gamma}: \mathfrak{X}_{\alpha \beta \gamma} \hookrightarrow \mathcal{P}_{\alpha \beta \gamma}$ induisant les morphismes canoniques au niveau des fibres spéciales. Sauf mention du contraire, tous ces relèvements seront supposés fixés par la suite.

Via les isomorphismes de la forme $\tau$ (2.1.10), on remarque que l'on dispose des diagrammes commutatifs de foncteurs suivants

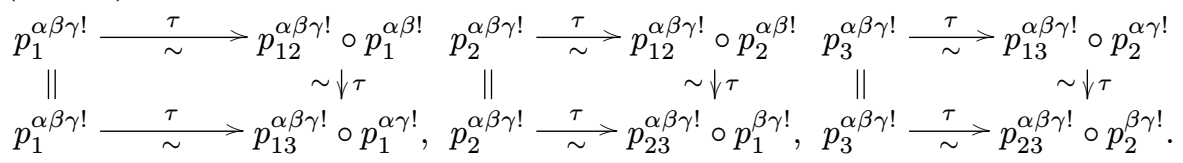

DÉfinition 2.5.2. - Pour tout $\alpha \in \Lambda$, donnons-nous $\mathcal{E}_{\alpha}$, un $\mathcal{D}_{\mathfrak{X}_{\alpha}}^{\dagger}\left({ }^{\dagger} T \cap X_{\alpha}\right) \mathbb{Q}^{-}$ module cohérent. On appelle donnée de recollement sur $\left(\mathcal{E}_{\alpha}\right)_{\alpha \in \Lambda}$, la donnée

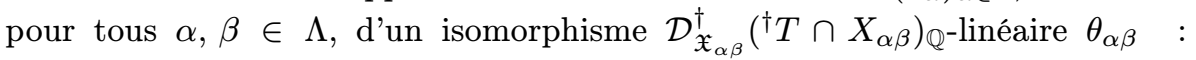
$p_{2}^{\alpha \beta !}\left(\mathcal{E}_{\beta}\right) \stackrel{\sim}{\longrightarrow} p_{1}^{\alpha \beta !}\left(\mathcal{E}_{\alpha}\right)$, ceux-ci vérifiant la condition de cocycle : $\theta_{13}^{\alpha \beta \gamma}=$ 
$\theta_{12}^{\alpha \beta \gamma} \circ \theta_{23}^{\alpha \beta \gamma}$, où $\theta_{12}^{\alpha \beta \gamma}, \theta_{23}^{\alpha \beta \gamma}$ et $\theta_{13}^{\alpha \beta \gamma}$ sont définis par les diagrammes commutatifs

$$
\begin{aligned}
& p_{12}^{\alpha \beta \gamma !} p_{2}^{\alpha \beta !}\left(\mathcal{E}_{\beta}\right) \stackrel{\tau}{\sim} p_{2}^{\alpha \beta \gamma !}\left(\mathcal{E}_{\beta}\right) \\
& \sim \downarrow p_{12}^{\alpha \beta \gamma !}\left(\theta_{\alpha \beta}\right) \quad \forall \theta_{12}^{\alpha \beta \gamma} \\
& p_{12}^{\alpha \beta \gamma !} p_{1}^{\alpha \beta !}\left(\mathcal{E}_{\alpha}\right) \stackrel{\tau}{\sim} \underset{\sim}{\sim} p_{1}^{\alpha \beta \gamma !}\left(\mathcal{E}_{\alpha}\right) \text {, } \\
& p_{23}^{\alpha \beta \gamma !} p_{2}^{\beta \gamma !}\left(\mathcal{E}_{\gamma}\right) \stackrel{\tau}{\sim} p_{3}^{\alpha \beta \gamma !}\left(\mathcal{E}_{\gamma}\right) \\
& \sim \downarrow p_{23}^{\alpha \beta \gamma !}\left(\theta_{\beta \gamma}\right) \quad \forall \theta_{23}^{\alpha \beta \gamma} \\
& p_{23}^{\alpha \beta \gamma !} p_{1}^{\beta \gamma !}\left(\mathcal{E}_{\beta}\right) \stackrel{\tau}{\sim} p_{2}^{\alpha \beta \gamma !}\left(\mathcal{E}_{\beta}\right) \text {, } \\
& p_{13}^{\alpha \beta \gamma !} p_{2}^{\alpha \gamma !}\left(\mathcal{E}_{\gamma}\right) \stackrel{\tau}{\sim} \underset{\sim}{\sim} p_{3}^{\alpha \beta \gamma !}\left(\mathcal{E}_{\gamma}\right) \\
& \sim \downarrow p_{13}^{\alpha \beta \gamma !}\left(\theta_{\alpha \gamma}\right) \quad \forall \theta_{13}^{\alpha \beta \gamma} \\
& p_{13}^{\alpha \beta \gamma !} p_{1}^{\alpha \gamma !}\left(\mathcal{E}_{\alpha}\right) \stackrel{\tau}{\sim} p_{1}^{\alpha \beta \gamma !}\left(\mathcal{E}_{\alpha}\right) \text {. }
\end{aligned}
$$

On construit la catégorie $\operatorname{Coh}\left(X,\left(\mathcal{P}_{\alpha}\right)_{\alpha \in \Lambda}, T\right)$ de la manière suivante :

- un objet est une famille $\left(\mathcal{E}_{\alpha}\right)_{\alpha \in \Lambda}$ de $\mathcal{D}_{\mathfrak{X}_{\alpha}}^{\dagger}\left({ }^{\dagger} T \cap X_{\alpha}\right)_{\mathbb{Q}^{-m o d u l e s ~ c o h e ́ r e n t s, ~}}$ $\mathcal{E}_{\alpha}$, munie d'une donnée de recollement $\left(\theta_{\alpha \beta}\right)_{\alpha, \beta \in \Lambda}$,

- un morphisme $\left(\left(\mathcal{E}_{\alpha}\right)_{\alpha \in \Lambda},\left(\theta_{\alpha \beta}\right)_{\alpha, \beta \in \Lambda}\right) \rightarrow\left(\left(\mathcal{E}_{\alpha}^{\prime}\right)_{\alpha \in \Lambda},\left(\theta_{\alpha \beta}^{\prime}\right)_{\alpha, \beta \in \Lambda}\right)$ est une famille de morphismes $f_{\alpha}: \mathcal{E}_{\alpha} \rightarrow \mathcal{E}_{\alpha}^{\prime}$ commutant aux données de recollement, i.e., telle que le diagramme suivant soit commutatif :

$$
\begin{gathered}
p_{2}^{\alpha \beta !}\left(\mathcal{E}_{\beta}\right) \stackrel{\theta_{\alpha \beta}}{\sim} p_{1}^{\alpha \beta !}\left(\mathcal{E}_{\alpha}\right) \\
\downarrow p_{2}^{\alpha \beta !}\left(f_{\beta}\right), \quad \downarrow p_{1}^{\alpha \beta !}\left(f_{\alpha}\right) \\
p_{2}^{\alpha \beta !}\left(\mathcal{E}_{\beta}^{\prime}\right) \stackrel{\theta_{\alpha \beta}}{\sim} p_{1}^{\alpha \beta !}\left(\mathcal{E}_{\alpha}^{\prime}\right) .
\end{gathered}
$$

Lorsque $T$ est vide, on omettra comme d'habitude de l'indiquer.

Remarques 2.5.3. - Pour tous $\alpha, \beta \in \Lambda$, soient $f_{\alpha}: \mathcal{E}_{\alpha} \rightarrow \mathcal{E}_{\alpha}^{\prime}$ un mor-

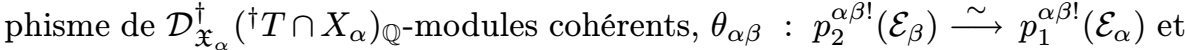
$\theta_{\alpha \beta}^{\prime}: p_{2}^{\alpha \beta !}\left(\mathcal{E}_{\beta}^{\prime}\right) \stackrel{\sim}{\longrightarrow} p_{1}^{\alpha \beta !}\left(\mathcal{E}_{\alpha}^{\prime}\right)$ des isomorphismes $\mathcal{D}_{\mathfrak{X}_{\alpha \beta}}^{\dagger}\left({ }^{\dagger} T \cap X_{\alpha \beta}\right) \mathbb{Q}_{\mathbb{Q}}$-linéaires. On suppose en outre que les morphismes $f_{\alpha}$ et les isomorphismes $\theta_{\alpha \beta}$ et $\theta_{\alpha \beta}^{\prime}$ induisent le diagramme commutatif 2.5.2.2.

Alors, les isomorphismes $\theta_{\alpha \beta}$ satisfont à la condition de cocycle si et seulement s'il en est de même des isomorphismes $\theta_{\alpha \beta}^{\prime}$. En effet, en transformant, via 2.5.2.2, les carrés 2.5.2.1 en trois cubes commutatifs, on obtient les trois carrés 
commutatifs suivants :

$$
\begin{aligned}
& p_{2}^{\alpha \beta \gamma !}\left(\mathcal{E}_{\beta}\right) \stackrel{p_{2}^{\alpha \beta \gamma !}\left(f_{\beta}\right)}{\longrightarrow} p_{2}^{\alpha \beta \gamma !}\left(\mathcal{E}_{\beta}^{\prime}\right) \\
& \sim \downarrow \theta_{12}^{\alpha \beta \gamma} p_{\alpha \beta \gamma !}^{\alpha \beta}\left(f_{\alpha}\right) \quad \sim \downarrow \theta_{12}^{\prime \alpha \beta \gamma} \\
& p_{1}^{\alpha \beta \gamma !}\left(\mathcal{E}_{\alpha}\right) \stackrel{p_{1}^{\alpha \beta \gamma !}\left(f_{\alpha}\right)}{\longrightarrow} p_{1}^{\alpha \beta \gamma !}\left(\mathcal{E}_{\alpha}^{\prime}\right), \\
& p_{3}^{\alpha \beta \gamma !}\left(\mathcal{E}_{\gamma}\right) \stackrel{p_{3}^{\alpha \beta \gamma !}\left(f_{\gamma}\right)}{\longrightarrow} p_{3}^{\alpha \beta \gamma !}\left(\mathcal{E}_{\gamma}^{\prime}\right) \\
& \sim \downarrow \theta_{23}^{\alpha \beta \gamma} p_{\alpha \beta \gamma !} \quad \sim \downarrow \theta_{23}^{\prime \alpha \beta \gamma} \\
& p_{2}^{\alpha \beta \gamma !}\left(\mathcal{E}_{\beta}\right) \stackrel{p_{2}^{\alpha \beta \gamma !}\left(f_{\beta}\right)}{\longrightarrow} p_{2}^{\alpha \beta \gamma !}\left(\mathcal{E}_{\beta}^{\prime}\right), \\
& p_{3}^{\alpha \beta \gamma !}\left(\mathcal{E}_{\gamma}\right) \stackrel{p_{3}^{\alpha \beta \gamma !}\left(f_{\gamma}\right)}{\longrightarrow} p_{3}^{\alpha \beta \gamma !}\left(\mathcal{E}_{\gamma}^{\prime}\right)
\end{aligned}
$$

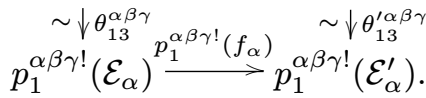

Proposition 2.5.4. - On dispose d'une équivalence de catégories entre $\operatorname{Coh}(X, \mathcal{P}, T)$ et $\operatorname{Coh}\left(X,\left(\mathcal{P}_{\alpha}\right)_{\alpha \in \Lambda}, T\right)$.

Démonstration. - Construisons d'abord le foncteur canonique $\mathcal{L}$ oc $: \operatorname{Coh}(X, \mathcal{P}, T) \rightarrow \operatorname{Coh}\left(X,\left(\mathcal{P}_{\alpha}\right)_{\alpha \in \Lambda}, T\right)$. Pour tout $\mathcal{D}_{\mathcal{P}, \mathbb{Q}}^{\dagger}\left({ }^{\dagger} T\right)$-module cohérent $\mathcal{E}$ à support dans $X$, on définit l'isomorphisme $\theta_{\alpha \beta}: p_{2}^{\alpha \beta !} u_{\beta}^{!}\left(\left.\mathcal{E}\right|_{\mathcal{P}_{\beta}}\right) \stackrel{\sim}{\longrightarrow}$ $p_{1}^{\alpha \beta !} u_{\alpha}^{!}\left(\left.\mathcal{E}\right|_{\mathcal{P}_{\alpha}}\right)$, comme étant l'unique flèche rendant commutatif le diagramme suivant

$$
\begin{gathered}
p_{2}^{\alpha \beta !} u_{\beta}^{!}\left(\left.\mathcal{E}\right|_{\mathcal{P}_{\beta}}\right) \stackrel{\tau}{\stackrel{\tau}{\sim}} u_{\alpha \beta}^{!}\left(\left.\left(\left.\mathcal{E}\right|_{\mathcal{P}_{\beta}}\right)\right|_{\mathcal{P}_{\alpha \beta}}\right) \\
\| \theta_{\alpha \beta} \\
p_{1}^{\alpha \beta !} u_{\alpha}^{!}\left(\left.\mathcal{E}\right|_{\mathcal{P}_{\alpha}}\right) \stackrel{\tau}{\stackrel{\tau}{\sim}} u_{\alpha \beta}^{!}\left(\left.\left(\left.\mathcal{E}\right|_{\mathcal{P}_{\alpha}}\right)\right|_{\mathcal{P}_{\alpha \beta}}\right) .
\end{gathered}
$$

Via l'isomorphisme $\tau: p_{12}^{\alpha \beta \gamma !} u_{\alpha \beta}^{!}\left(\left.\left(\left.\mathcal{E}\right|_{\mathcal{P}_{\alpha}}\right)\right|_{\mathcal{P}_{\alpha \beta}}\right) \stackrel{\sim}{\longrightarrow} u_{\alpha \beta \gamma}^{!}\left(\left.\left(\left.\mathcal{E}\right|_{\mathcal{P}_{\beta}}\right)\right|_{\mathcal{P}_{\alpha \beta \gamma}}\right)$, en appliquant le foncteur $p_{12}^{\alpha \beta \gamma \text { ! }}$ au carré 2.5.4.1 et avec 2.5.2.1, on obtient le diagramme commutatif :

$$
\begin{gathered}
p_{2}^{\alpha \beta \gamma !}\left(u_{\beta}^{!}\left(\left.\mathcal{E}\right|_{\mathcal{P}_{\beta}}\right)\right) \stackrel{\tau}{\stackrel{\tau}{\sim}} \underset{\sim}{\sim} u_{\alpha \beta \gamma}^{!}\left(\left.\left(\left.\mathcal{E}\right|_{\mathcal{P}_{\beta}}\right)\right|_{\mathcal{P}_{\alpha \beta \gamma}}\right) \\
\quad \sim \downarrow \theta_{12}^{\alpha \beta \gamma} \\
p_{1}^{\alpha \beta \gamma !}\left(u_{\alpha}^{!}\left(\left.\mathcal{E}\right|_{\mathcal{P}_{\alpha}}\right)\right) \stackrel{\tau}{\stackrel{\tau}{\sim}} \underset{\sim}{\sim} u_{\alpha \beta \gamma}^{!}\left(\left.\left(\left.\mathcal{E}\right|_{\mathcal{P}_{\alpha}}\right)\right|_{\mathcal{P}_{\alpha \beta \gamma}}\right),
\end{gathered}
$$

où les isomorphismes horizontaux sont de la forme $\tau$, grâce à la formule de transitivité et à la commutation aux images inverses extraordinaires des isomorphismes de la forme $\tau$ (2.1.10). De même, on construit les deux autres diagrammes analogues. Avec ces trois-ci, on vérifie que $\mathcal{L} \operatorname{oc}(\mathcal{E}):=\left(\left(u_{\alpha}^{!}\left(\left.\mathcal{E}\right|_{\mathcal{P}_{\alpha}}\right)\right)_{\alpha \in \Lambda},\left(\theta_{\alpha \beta}\right)_{\alpha, \beta \in \Lambda}\right)$ satisfait à la condition de cocycle et est ainsi un objet de $\operatorname{Coh}\left(X,\left(\mathcal{P}_{\alpha}\right)_{\alpha \in \Lambda}, T\right)$. 
En outre, si $f: \mathcal{E} \rightarrow \mathcal{E}^{\prime}$ est un morphisme de $\mathcal{D}_{\mathcal{P}}^{\dagger}\left({ }^{\dagger} T\right)_{\mathbb{Q}}$-modules cohérents à support dans $X$, alors, par fonctorialité en $\mathcal{E}$ de 2.5.4.1 (on transforme le carré 2.5.4.1 en cube), la famille $\left(u_{\alpha}^{!}\left(\left.f\right|_{\mathcal{P}_{\alpha}}\right)\right)_{\alpha \in \Lambda}$ commute aux données de recollement.

Construisons à présent un foncteur quasi-inverse canonique $\mathcal{R} e c o l$ : $\operatorname{Coh}\left(X,\left(\mathcal{P}_{\alpha}\right)_{\alpha \in \Lambda}, T\right) \rightarrow \operatorname{Coh}(X, \mathcal{P}, T)$.

Soit $\left(\mathcal{E}_{\alpha}\right)_{\alpha \in \Lambda}$ une famille de $\mathcal{D}_{\mathfrak{X}_{\alpha}, \mathbb{Q}}^{\dagger}\left({ }^{\dagger} T \cap X_{\alpha}\right)$-modules cohérents munie d'une donnée de recollement $\left(\theta_{\alpha \beta}\right)_{\alpha, \beta \in \Lambda}$. Prouvons que la famille $\left(u_{\alpha+}\left(\mathcal{E}_{\alpha}\right)\right)_{\alpha \in \Lambda}$ se recolle en un $\mathcal{D}_{\mathcal{P}, \mathbb{Q}}^{\dagger}\left({ }^{\dagger} T\right)$-module cohérent à support dans $X$.

Pour cela, notons $\phi_{1}^{\alpha \beta}$ (resp. $\phi_{2}^{\alpha \beta}$ ) le morphisme d'adjonction (voir 2.2.2) du carré de gauche (resp. de droite)
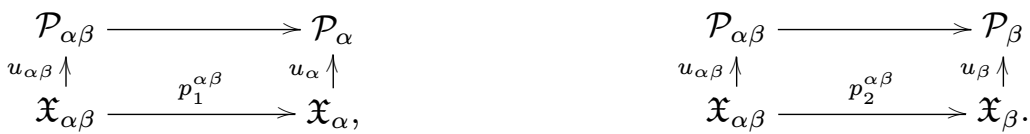

On construit pour tous $\alpha, \beta \in \Lambda$ un isomorphisme $\tau_{\alpha \beta}:\left.\left(u_{\beta+}\left(\mathcal{E}_{\beta}\right)\right)\right|_{\mathcal{P}_{\alpha \beta}} \stackrel{\sim}{\longrightarrow}$ $\left.\left(u_{\alpha+}\left(\mathcal{E}_{\alpha}\right)\right)\right|_{\mathcal{P}_{\alpha \beta}}$ comme étant l'unique morphisme rendant commutatif le diagramme suivant

$$
\begin{array}{cc}
\left.u_{\alpha \beta+} \circ p_{1}^{\alpha \beta !}\left(\mathcal{E}_{\alpha}\right) \frac{\phi_{1}^{\alpha \beta}\left(\mathcal{E}_{\alpha}\right)}{\sim}\left(u_{\alpha+}\left(\mathcal{E}_{\alpha}\right)\right)\right|_{\mathcal{P}_{\alpha \beta}} \\
u_{\alpha \beta+}\left(\theta_{\alpha \beta}\right) \uparrow \sim \\
\left.\tau_{\alpha \beta \beta} \sim \circ p_{2}^{\alpha \beta !}\left(\mathcal{E}_{\beta}\right) \frac{\phi_{2}^{\alpha \beta}\left(\mathcal{E}_{\beta}\right)}{\sim}\left(u_{\beta+}\left(\mathcal{E}_{\beta}\right)\right)\right|_{\mathcal{P}_{\alpha \beta}} .
\end{array}
$$

Il reste maintenant à établir que les isomorphismes $\tau_{\alpha \beta}$ vérifient la condition de recollement. À cette fin, notons $\phi_{12}^{\alpha \beta \gamma}$ (resp. $\phi_{23}^{\alpha \beta \gamma}$ et $\phi_{13}^{\alpha \beta \gamma}$ ) le morphisme d'adjonction (toujours 2.2.2) du carré de gauche (resp. du centre et de droite) suivant

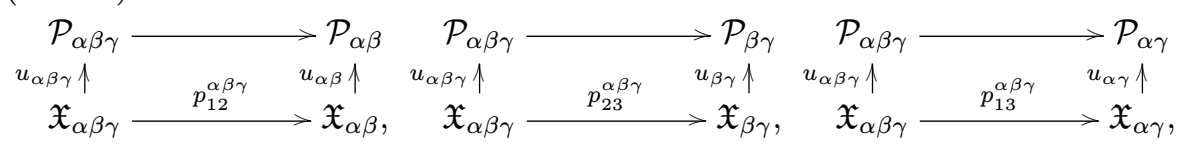

et $\phi_{1}^{\alpha \beta \gamma}$ (resp. $\phi_{2}^{\alpha \beta \gamma}$ et $\phi_{3}^{\alpha \beta \gamma}$ ) celui du diagramme de gauche (resp. du centre et de droite) :

$$
\begin{aligned}
& \mathcal{P}_{\alpha \beta \gamma} \longrightarrow \mathcal{P}_{\alpha} \quad \mathcal{P}_{\alpha \beta \gamma} \longrightarrow \mathcal{P}_{\beta} \quad \mathcal{P}_{\alpha \beta \gamma} \longrightarrow \mathcal{P}_{\gamma} \\
& \begin{array}{ccccc}
u_{\alpha \beta \gamma} \uparrow & p_{1}^{\alpha \beta \gamma} u_{\alpha} \uparrow & u_{\alpha \beta \gamma} \uparrow & p_{2}^{\alpha \beta \gamma} u_{\beta} \uparrow & u_{\alpha \beta \gamma \uparrow} \\
\mathfrak{X}_{\alpha \beta \gamma} \stackrel{\mathfrak{X}_{\alpha}}{\longrightarrow}, & \mathfrak{X}_{\alpha \beta \gamma} \stackrel{p_{\beta}^{\alpha \beta \gamma},}{\mathfrak{X}_{\gamma} \uparrow} & \mathfrak{X}_{\alpha \beta \gamma} \stackrel{\mathcal{X}_{3}}{\longrightarrow} \mathfrak{X}_{\gamma} .
\end{array}
\end{aligned}
$$


Considérons le diagramme commutatif suivant

$$
\begin{aligned}
& \left.u_{\alpha \beta \gamma+} p_{1}^{\alpha \beta \gamma !}\left(\mathcal{E}_{\alpha}\right) \stackrel{u_{\alpha \beta \gamma+}(\tau)}{\sim} u_{\alpha \beta \gamma+} \circ p_{12}^{\alpha \beta \gamma !}\left(p_{1}^{\alpha \beta !}\left(\mathcal{E}_{\alpha}\right)\right) \stackrel{\phi_{12}^{\alpha \beta \gamma}\left(p_{1}^{\alpha \beta !}\left(\mathcal{E}_{\alpha}\right)\right)}{\sim} u_{\alpha \beta+}\left(p_{1}^{\alpha \beta !}\left(\mathcal{E}_{\alpha}\right)\right)\right|_{\mathcal{P}_{\alpha \beta \gamma}} \\
& \left.u_{\alpha \beta \gamma+}\left(\theta_{12}^{\alpha \beta \gamma}\right) \uparrow \sim \quad u_{\alpha \beta \gamma+} \circ p_{12}^{\alpha \beta \gamma !}\left(\theta_{\alpha \beta}\right) \uparrow \sim \quad \sim u_{\alpha \beta \gamma+}(\tau) u_{\alpha \beta+}\left(\theta_{\alpha \beta}\right)\right|_{\mathcal{P}_{\alpha \beta \gamma}} \\
& \left.u_{\alpha \beta \gamma+} p_{2}^{\alpha \beta \gamma !}\left(\mathcal{E}_{\beta}\right) \stackrel{u_{\alpha \beta \gamma+}(\tau)}{\sim} u_{\alpha \beta \gamma+} \circ p_{12}^{\alpha \beta \gamma !}\left(p_{2}^{\alpha \beta !}\left(\mathcal{E}_{\beta}\right)\right) \stackrel{\phi_{12}^{\alpha \beta \gamma}\left(p_{2}^{\alpha \beta !}\left(\mathcal{E}_{\beta}\right)\right)}{\sim} u_{\alpha \beta+}\left(p_{2}^{\alpha \beta !}\left(\mathcal{E}_{\beta}\right)\right)\right|_{\mathcal{P}_{\alpha \beta \gamma}} .
\end{aligned}
$$

Grâce à 2.2.2.i) et 2.2.2.ii), on obtient alors

$$
\begin{aligned}
& \left(\left.\phi_{1}^{\alpha \beta}\left(\mathcal{E}_{\alpha}\right)\right|_{\mathcal{P}_{\alpha \beta \gamma}}\right) \circ \phi_{12}^{\alpha \beta \gamma}\left(p_{1}^{\alpha \beta !}\left(\mathcal{E}_{\alpha}\right)\right) \circ u_{\alpha \beta \gamma+}(\tau)=\phi_{1}^{\alpha \beta \gamma}\left(\mathcal{E}_{\alpha}\right), \\
& \left(\left.\phi_{2}^{\alpha \beta}\left(\mathcal{E}_{\beta}\right)\right|_{\mathcal{P}_{\alpha \beta \gamma}}\right) \circ \phi_{12}^{\alpha \beta \gamma}\left(p_{2}^{\alpha \beta !}\left(\mathcal{E}_{\beta}\right)\right) \circ u_{\alpha \beta \gamma+}(\tau)=\phi_{2}^{\alpha \beta \gamma}\left(\mathcal{E}_{\beta}\right), \\
& \left(\left.\phi_{1}^{\beta \gamma}\left(\mathcal{E}_{\beta}\right)\right|_{\mathcal{P}_{\alpha \beta \gamma}}\right) \circ \phi_{23}^{\alpha \beta \gamma}\left(p_{1}^{\beta \gamma !}\left(\mathcal{E}_{\beta}\right)\right) \circ u_{\alpha \beta \gamma+}(\tau)=\phi_{2}^{\alpha \beta \gamma}\left(\mathcal{E}_{\beta}\right), \\
& \left(\left.\phi_{2}^{\beta \gamma}\left(\mathcal{E}_{\gamma}\right)\right|_{\mathcal{P}_{\alpha \beta \gamma}}\right) \circ \phi_{23}^{\alpha \beta \gamma}\left(p_{2}^{\beta \gamma !}\left(\mathcal{E}_{\gamma}\right)\right) \circ u_{\alpha \beta \gamma+}(\tau)=\phi_{3}^{\alpha \beta \gamma}\left(\mathcal{E}_{\gamma}\right), \\
& \left(\left.\phi_{1}^{\alpha \gamma}\left(\mathcal{E}_{\alpha}\right)\right|_{\mathcal{P}_{\alpha \beta \gamma}}\right) \circ \phi_{13}^{\alpha \beta \gamma}\left(p_{1}^{\alpha \gamma !}\left(\mathcal{E}_{\alpha}\right)\right) \circ u_{\alpha \beta \gamma+}(\tau)=\phi_{1}^{\alpha \beta \gamma}\left(\mathcal{E}_{\alpha}\right), \\
& \left(\left.\phi_{2}^{\alpha \gamma}\left(\mathcal{E}_{\gamma}\right)\right|_{\mathcal{P}_{\alpha \beta \gamma}}\right) \circ \phi_{13}^{\alpha \beta \gamma}\left(p_{2}^{\alpha \gamma !}\left(\mathcal{E}_{\gamma}\right)\right) \circ u_{\alpha \beta \gamma+}(\tau)=\phi_{3}^{\alpha \beta \gamma}\left(\mathcal{E}_{\gamma}\right) .
\end{aligned}
$$

En composant 2.5.4.3 restreint à $\mathcal{P}_{\alpha \beta \gamma}$ et 2.5.4.6, via les égalités 2.5.4.7 et 2.5.4.8, on obtient le carré commutatif :

$$
\begin{array}{cr}
u_{\alpha \beta \gamma+} p_{1}^{\alpha \beta \gamma !}\left(\mathcal{E}_{\alpha}\right) \frac{\phi_{1}^{\alpha \beta \gamma}\left(\mathcal{E}_{\alpha}\right)}{\sim} & \left.\left(u_{\alpha+}\left(\mathcal{E}_{\alpha}\right)\right)\right|_{\mathcal{P}_{\alpha \beta \gamma}} \\
u_{\alpha \beta \gamma+}\left(\theta_{12}^{\alpha \beta \gamma}\right) \uparrow \sim & \left.\sim \uparrow \tau_{\alpha \beta}\right|_{\mathcal{P}_{\alpha \beta \gamma}} \\
u_{\alpha \beta \gamma+} p_{2}^{\alpha \beta \gamma !}\left(\mathcal{E}_{\beta}\right) \stackrel{\phi_{2}^{\alpha \beta \gamma}\left(\mathcal{E}_{\beta}\right)}{\sim} & \left.\sim\left(u_{\beta+}\left(\mathcal{E}_{\beta}\right)\right)\right|_{\mathcal{P}_{\alpha \beta \gamma}} .
\end{array}
$$

De façon analogue, en utilisant 2.5.4.9 et 2.5.4.10 (resp. 2.5.4.11 et 2.5.4.12) on obtient les diagrammes commutatifs suivants :

$(2.5 .4 .14)$

$$
\begin{gathered}
\left.\left.u_{\alpha \beta \gamma+} \circ p_{2}^{\alpha \beta \gamma !}\left(\mathcal{E}_{\beta}\right) \stackrel{\phi_{2}^{\alpha \beta \gamma}\left(\mathcal{E}_{\beta}\right)}{\sim}\left(u_{\beta+}\left(\mathcal{E}_{\beta}\right)\right)\right|_{\mathcal{P}_{\alpha \beta \gamma}} \quad u_{\alpha \beta \gamma+} \circ p_{1}^{\alpha \beta \gamma !}\left(\mathcal{E}_{\alpha}\right) \stackrel{\phi_{1}^{\alpha \beta \gamma}\left(\mathcal{E}_{\alpha}\right)}{\sim}\left(u_{\alpha+}\left(\mathcal{E}_{\alpha}\right)\right)\right|_{\mathcal{P}_{\alpha \beta \gamma}} \\
\sim \uparrow \tau_{\beta \gamma \mid \mathcal{P}_{\alpha \beta \gamma}} \\
u_{\alpha \beta \gamma+}\left(\theta_{13}^{\alpha \beta \gamma}\right) \uparrow \sim
\end{gathered}
$$

De ces trois derniers diagrammes, comme le foncteur $u_{\alpha \beta \gamma+}$ est (pleinement) fidèle (pour les modules cohérents), il en dérive que les isomorphismes $\theta_{\alpha \beta}$ vérifient la condition de cocycle si et seulement si les isomorphismes $\tau_{\alpha \beta}$ se recollent.

Soit $f=\left(f_{\alpha}\right)_{\alpha \in \Lambda}:\left(\left(\mathcal{E}_{\alpha}\right)_{\alpha \in \Lambda},\left(\theta_{\alpha \beta}\right)_{\alpha, \beta \in \Lambda}\right) \rightarrow\left(\left(\mathcal{E}_{\alpha}^{\prime}\right)_{\alpha \in \Lambda},\left(\theta_{\alpha \beta}^{\prime}\right)_{\alpha, \beta \in \Lambda}\right)$, un morphisme de $\operatorname{Coh}\left(X,\left(\mathcal{P}_{\alpha}\right)_{\alpha \in \Lambda}, T\right)$. On lui associe la famille $\operatorname{Recol}(f)$ : $\left(u_{\alpha+}\left(f_{\alpha}\right)\right)_{\alpha \in \Lambda}$. En notant $\tau_{\alpha \beta}$ (resp. $\left.\tau_{\alpha \beta}^{\prime}\right)$ l'isomorphisme rendant commutatif 
2.5.4.3 pour $\theta_{\alpha \beta}$ (resp. $\left.\theta_{\alpha \beta}^{\prime}\right)$, on obtient le cube

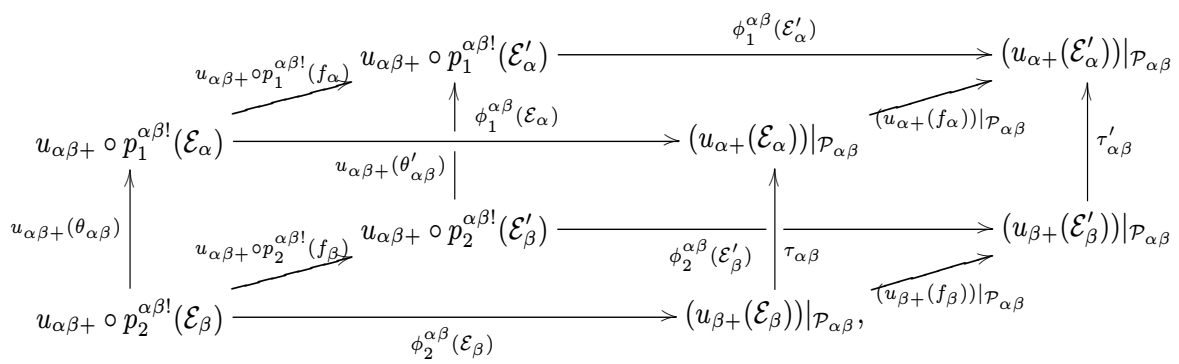

dont les carrés de devant, de derrière, du bas et du haut sont commutatifs par fonctorialité ou grâce à 2.5.4.3. Comme celui de gauche l'est (via 2.5.2.2), il en résulte qu'il en est de même du carré de droite. Les morphismes $u_{\alpha+}\left(f_{\alpha}\right)$ se recollent donc.

On a donc construit le foncteur Recol. Prouvons maintenant que celui-ci est quasi-inverse de $\mathcal{L}$ oc. Soit $\left(\left(\mathcal{E}_{\alpha}\right)_{\alpha \in \Lambda},\left(\theta_{\alpha \beta}\right)_{\alpha, \beta \in \Lambda}\right)$ un objet de $\operatorname{Coh}\left(X,\left(\mathcal{P}_{\alpha}\right)_{\alpha \in \Lambda}, T\right)$. Dans un premier temps, il s'agit d'établir un isomorphisme fonctoriel $\operatorname{Loc} \circ \mathcal{R} \operatorname{ecol}\left(\left(\mathcal{E}_{\alpha}\right)_{\alpha \in \Lambda},\left(\theta_{\alpha \beta}\right)_{\alpha, \beta \in \Lambda}\right) \stackrel{\sim}{\longrightarrow}\left(\left(\mathcal{E}_{\alpha}\right)_{\alpha \in \Lambda},\left(\theta_{\alpha \beta}\right)_{\alpha, \beta \in \Lambda}\right)$.

Notons $\mathcal{E}:=\mathcal{R} \operatorname{ecol}\left(\left(\mathcal{E}_{\alpha}\right)_{\alpha \in \Lambda},\left(\theta_{\alpha \beta}\right)_{\alpha, \beta \in \Lambda}\right), \mathcal{L} o c(\mathcal{E})=\left(\left(u_{\alpha}^{!}\left(\left.\mathcal{E}\right|_{\mathcal{P}_{\alpha}}\right)\right)_{\alpha \in \Lambda},\left(\theta_{\alpha \beta}^{\prime \prime}\right)_{\alpha, \beta \in \Lambda}\right)$ et $\tau_{\alpha}:\left.u_{\alpha+}\left(\mathcal{E}_{\alpha}\right) \stackrel{\sim}{\longrightarrow} \mathcal{E}\right|_{\mathcal{P}_{\alpha}}$ les isomorphismes canoniques vérifiant $\tau_{\alpha \beta}=$ $\left.\left.\tau_{\alpha}^{-1}\right|_{\mathcal{P}_{\alpha \beta}} \circ \tau_{\beta}\right|_{\mathcal{P}_{\alpha \beta}}$, où $\tau_{\alpha \beta}$ a été défini via 2.5.4.3. Considérons le diagramme suivant

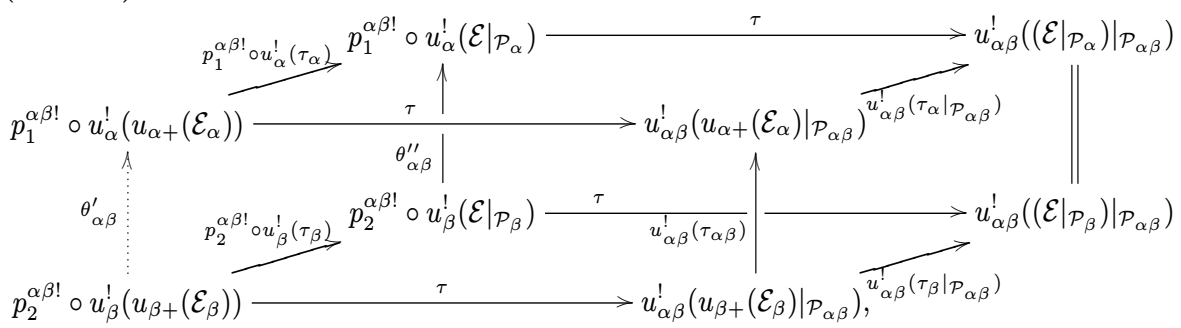

où la flèche $\theta_{\alpha \beta}^{\prime}$ est par définition celle rendant commutatif le carré de devant. Les carrés du fond et de droite sont commutatifs par définition et ceux du haut et du bas le sont par fonctorialité. Grâce la remarque 2.5.3, on en déduit que les isomorphismes $\theta_{\alpha \beta}^{\prime}$ vérifie la condition de cocycle et on se ramène à prouver que l'isomorphisme d'adjonction $\mathcal{E}_{\alpha} \stackrel{\sim}{\longrightarrow} u_{\alpha}^{!} \circ u_{\alpha+}\left(\mathcal{E}_{\alpha}\right)$ est compatible aux données 
de recollement respectives, i.e., que le carré de gauche suivant

$$
\begin{gathered}
p_{1}^{\alpha \beta !}\left(\mathcal{E}_{\alpha}\right) \underset{\operatorname{adj}_{u_{\alpha}}}{\sim} p_{1}^{\alpha \beta !} \circ u_{\alpha}^{!} \circ u_{\alpha+}\left(\mathcal{E}_{\alpha}\right) \stackrel{\tau}{\sim} u_{\alpha \beta}^{!}\left(\left.u_{\alpha+}\left(\mathcal{E}_{\alpha}\right)\right|_{\mathcal{P}_{\alpha \beta}}\right) \\
\theta_{\alpha \beta \uparrow \sim} \sim u_{\alpha \beta}^{\prime}\left(\tau_{\alpha \beta}\right) \uparrow \sim \\
p_{2}^{\alpha \beta !}\left(\mathcal{E}_{\beta}\right) \underset{\operatorname{adj}_{u_{\beta}}}{\sim} \underset{\sim}{\sim} p_{2}^{\alpha \beta !} \circ u_{\beta}^{!} \circ u_{\beta+}\left(\mathcal{E}_{\beta}\right) \stackrel{\tau}{\sim} u_{\alpha \beta}^{!}\left(\left.u_{\beta+}\left(\mathcal{E}_{\beta}\right)\right|_{\mathcal{P}_{\alpha \beta}}\right)
\end{gathered}
$$

est commutatif. Or, en appliquant le foncteur $u_{\alpha \beta+}$ au diagramme 2.5.4.17 et en composant avec le diagramme commutatif

$$
\begin{aligned}
& \left.u_{\alpha \beta+} u_{\alpha \beta}^{!}\left(\left.u_{\alpha+}\left(\mathcal{E}_{\alpha}\right)\right|_{\mathcal{P}_{\alpha \beta}}\right) \frac{\operatorname{adj}_{u_{\alpha \beta}}}{\sim} u_{\alpha+}\left(\mathcal{E}_{\alpha}\right)\right|_{\mathcal{P}_{\alpha \beta}} \\
& \begin{array}{cc}
u_{\alpha \beta+} u_{\alpha \beta}^{!}\left(\tau_{\alpha \beta}\right) \uparrow \sim & \tau_{\alpha \beta} \uparrow \sim \\
\left.u_{\alpha \beta+} u_{\alpha \beta}^{!}\left(\left.u_{\beta+}\left(\mathcal{E}_{\beta}\right)\right|_{\mathcal{P}_{\alpha \beta}}\right) \stackrel{\operatorname{adj}_{u_{\alpha \beta}}}{\sim} u_{\beta+}\left(\mathcal{E}_{\beta}\right)\right|_{\mathcal{P}_{\alpha \beta}},
\end{array}
\end{aligned}
$$

on obtient 2.5.4.3 (par construction de la flèche d'adjonction de la proposition 2.2.2), qui est commutatif. Le foncteur $u_{\alpha \beta+}$ étant fidèle, on démontre ainsi la commutativité du contour de 2.5.4.17. Puisque le carré de droite de 2.5.4.17 est commutatif (il correspond au carré de devant de 2.5.4.16), il en découle celle du carré gauche.

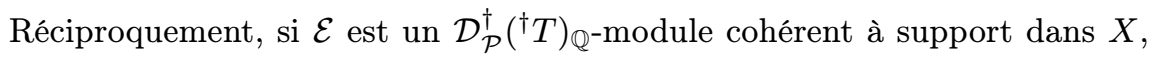
vérifions que l'on dispose d'un isomorphisme $\operatorname{Recol} \circ \mathcal{L}$ oc $(\mathcal{E}) \stackrel{\sim}{\longrightarrow} \mathcal{E}$ fonctoriel en $\mathcal{E}$. Notons $\left(\theta_{\alpha \beta}\right)_{\alpha \beta \in \Lambda}$, la donnée de recollement de $\left(u_{\alpha}^{!}\left(\left.\mathcal{E}\right|_{\mathcal{P}_{\alpha}}\right)\right)_{\alpha \in \Lambda}$ définie dans 2.5.4.1 et $\left(\tau_{\alpha \beta}\right)_{\alpha \beta \in \Lambda}$, la donnée de recollement de $\left(u_{\alpha+} u_{\alpha}^{!}\left(\left.\mathcal{E}\right|_{\mathcal{P}_{\alpha}}\right)\right)_{\alpha \in \Lambda}$ déduite de celle de $\left(u_{\alpha}^{!}\left(\left.\mathcal{E}\right|_{\mathcal{P}_{\alpha}}\right)\right)_{\alpha \in \Lambda}$ via 2.5.4.3. Prouvons maintenant que les isomorphismes d'adjonction $\left.u_{\alpha+} u_{\alpha}^{!}\left(\left.\mathcal{E}\right|_{\mathcal{P}_{\alpha}}\right) \rightarrow \mathcal{E}\right|_{\mathcal{P}_{\alpha}}$ sont compatibles aux données de recollement respectives, i.e., que le carré de droite suivant (la commutativité des deux autres est tautologique)

$$
\begin{aligned}
& \left.\left.u_{\alpha \beta+} \circ u_{\alpha \beta}^{!}\left(\left.\mathcal{E}\right|_{\mathcal{P}_{\alpha \beta}}\right) \stackrel{u_{\alpha \beta+}(\tau)}{\sim} u_{\alpha \beta+} \circ p_{1}^{\alpha \beta !}\left(u_{\alpha}^{!}\left(\left.\mathcal{E}\right|_{\mathcal{P}_{\alpha}}\right)\right) \frac{\phi_{1}^{\alpha \beta}\left(u_{\alpha}^{!}\left(\left.\mathcal{E}\right|_{\mathcal{P}_{\alpha}}\right)\right)}{\sim}\left(u_{\alpha+} u_{\alpha}^{!}\left(\left.\mathcal{E}\right|_{\mathcal{P}_{\alpha}}\right)\right)\right|_{\mathcal{P}_{\alpha \beta}} \stackrel{\left.\operatorname{adj}_{u_{\alpha}}\right|_{\mathcal{P}_{\alpha \beta}}}{\sim}\right|_{\mathcal{P}_{\alpha \beta}} \\
& \left.u_{\alpha \beta+} \circ u_{\alpha \beta}^{!}\left(\left.\mathcal{E}\right|_{\mathcal{P}_{\alpha \beta}}\right) \stackrel{u_{\alpha \beta+}(\tau)}{\sim} u_{\alpha \beta+} u_{\alpha \beta+} \circ p_{2}^{\alpha \beta !}\left(u_{\beta}^{!}\left(\left.\mathcal{E}\right|_{\mathcal{P}_{\beta}}\right)\right) \stackrel{\phi_{2}^{\alpha \beta}\left(u_{\beta}^{!}\left(\left.\mathcal{E}\right|_{\mathcal{P}_{\beta}}\right)\right)}{\sim}\left(\begin{array}{c}
\tau_{\alpha \beta} \uparrow \sim \\
\sim
\end{array} u_{\beta+} u_{\beta}^{!}\left(\left.\mathcal{E}\right|_{\mathcal{P}_{\beta}}\right)\right)\right|_{\mathcal{P}_{\alpha \beta}} \stackrel{\left.\operatorname{adj}_{u_{\beta}}\right|_{\mathcal{P}_{\alpha \beta}}}{\sim} \|\left.\right|_{\mathcal{P}_{\alpha \beta}}
\end{aligned}
$$

est commutatif. Or, on dispose du diagramme commutatif suivant :

$$
\begin{aligned}
& u_{\alpha \beta+} p_{1}^{\alpha \beta !} u_{\alpha}^{!}\left(\left.\mathcal{E}\right|_{\mathcal{P}_{\alpha}}\right) \stackrel{\operatorname{adj}_{u_{\alpha}}}{\sim} u_{\alpha \beta+} p_{1}^{\alpha \beta !} u_{\alpha}^{!} u_{\alpha+} u_{\alpha}^{!}\left(\left.\left.\left.\mathcal{E}\right|_{\mathcal{P}_{\alpha}} \stackrel{u_{\alpha \beta+}}{\stackrel{\sim}{\sim}} u_{\alpha \beta+} u_{\alpha \beta}^{!}\left(u_{\alpha+} u_{\alpha}^{!}\left(\left.\mathcal{E}\right|_{\mathcal{P}_{\alpha}}\right)\right)\right|_{\mathcal{P}_{\alpha \beta}} \stackrel{\operatorname{adj}_{u_{\alpha \beta}}}{\sim} u_{\alpha+} u_{\alpha}^{!}\left(\left.\mathcal{E}\right|_{\mathcal{P}_{\alpha}}\right)\right|_{\mathcal{P}_{\alpha \beta}}\right.
\end{aligned}
$$

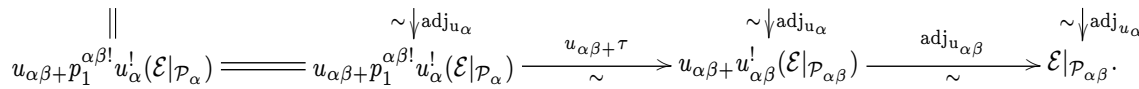

En effet, les deux carrés de droite de 2.5.4.19 sont commutatifs par fonctorialité, tandis que celui de gauche l'est pour les mêmes raisons que celle du deuxième carré de gauche de la troisième ligne de 2.2.2.1. Or, le morphisme $\phi_{1}^{\alpha \beta}\left(u_{\alpha}^{!}\left(\left.\mathcal{E}\right|_{\mathcal{P}_{\alpha}}\right)\right)$ 
est, par construction (voir la preuve de 2.2.2), égal au morphisme composé horizontal du haut de 2.5.4.19. Via la commutativité du diagramme 2.5.4.19, il en découle que le morphisme composé horizontal du haut de 2.5.4.18 est le morphisme d'adjonction $\left.u_{\alpha \beta+} u_{\alpha \beta}^{!}\left(\left.\mathcal{E}\right|_{\mathcal{P}_{\alpha \beta}}\right) \rightarrow \mathcal{E}\right|_{\mathcal{P}_{\alpha \beta}}$. De même, on vérifie que morphisme composé horizontal du bas de 2.5.4.18 est égal au morphisme d'adjonction par $u_{\alpha \beta}$.

REMARQUes 2.5.5. - Nous avons en fait prouvé mieux que 2.5.4 : nous avons construit un foncteur canonique $\mathcal{R}$ ecol quasi-inverse du foncteur canonique $\mathcal{L}$ oc : $\operatorname{Coh}(X, \mathcal{P}, T) \rightarrow \operatorname{Coh}\left(X,\left(\mathcal{P}_{\alpha}\right)_{\alpha \in \Lambda}, T\right)$. Cela correspond à une extension de l'analogue $p$-adique de Berthelot du théorème de Kashiwara (voir [6, 5.3.3]) qui correspond au cas où $X \hookrightarrow P$ se relève en un morphisme $u: \mathfrak{X} \hookrightarrow \mathcal{P}$ de $\mathcal{V}$-schémas formels lisses. Le foncteur $\mathcal{L} o c$ (resp. $\mathcal{R}$ ecol) étend d'une certaine manière $u^{!}$(resp. $\left.u_{+}\right)$.

DÉfinition 2.5.6. - On définit la catégorie $\operatorname{Isoc}^{\dagger}\left(Y, X,\left(\mathcal{P}_{\alpha}\right)_{\alpha \in \Lambda} / K\right)$ de la manière suivante : les objets sont les familles $\left(E_{\alpha}\right)_{\alpha \in \Lambda}$, de $j_{\alpha}^{\dagger} \mathcal{O}_{\mathfrak{X}_{\alpha} K^{-}}$ modules cohérents $E_{\alpha}$ possédant une connexion intégrable surconvergente, ces familles étant munies d'une donnée de recollement, i.e., d'isomorphismes, $\eta_{\alpha \beta}: p_{2 K}^{\alpha \beta !}\left(E_{\beta}\right) \stackrel{\sim}{\longrightarrow} p_{1 K}^{\alpha \beta !}\left(E_{\alpha}\right), j_{\alpha \beta}^{\dagger} \mathcal{D}_{\mathfrak{X}_{\alpha \beta K}}$-linéaires et vérifiant la condition de cocycle : $\eta_{13}^{\alpha \beta \gamma}=\eta_{12}^{\alpha \beta \gamma} \circ \eta_{23}^{\alpha \beta \gamma}$, où $\eta_{12}^{\alpha \beta \gamma}, \eta_{23}^{\alpha \beta \gamma}$ et $\eta_{13}^{\alpha \beta \gamma}$ sont définis par les diagrammes commutatifs

$$
\begin{aligned}
& p_{12 K}^{\alpha \beta \gamma !} p_{2 K}^{\alpha \beta !}\left(E_{\beta}\right) \stackrel{\epsilon}{\underset{\sim}{\sim}} p_{2 K}^{\alpha \beta \gamma !}\left(E_{\beta}\right) p_{23 K}^{\alpha \beta \gamma !} p_{2 K}^{\beta \gamma !}\left(E_{\gamma}\right) \stackrel{\epsilon}{\underset{\sim}{\sim}} p_{3 K}^{\alpha \beta \gamma !}\left(E_{\gamma}\right) p_{13 K}^{\alpha \beta \gamma !} p_{2 K}^{\alpha \gamma !}\left(E_{\gamma}\right) \stackrel{\epsilon}{\sim} p_{3 K}^{\alpha \beta \gamma !}\left(E_{\gamma}\right) \\
& \sim \downarrow p_{12 K}^{\alpha \beta \gamma}\left(\eta_{\alpha \beta}\right) \quad \forall \eta_{12}^{\alpha \beta \gamma} \quad \sim \downarrow p_{23 K}^{\alpha \beta \gamma !}\left(\eta_{\beta \gamma}\right) \quad \forall \eta_{23}^{\alpha \beta \gamma} \quad \sim \vee p_{13 K}^{\alpha \beta \gamma !}\left(\eta_{\alpha \gamma}\right) \quad \forall \eta_{13}^{\alpha \beta \gamma} \\
& p_{12 K}^{\alpha \beta \gamma !} p_{1 K}^{\alpha \beta !}\left(E_{\alpha}\right) \stackrel{\epsilon}{\underset{\sim}{\sim}} p_{1 K}^{\alpha \beta \gamma !}\left(E_{\alpha}\right), p_{23 K}^{\alpha \beta \gamma !} p_{1 K}^{\beta \gamma !}\left(E_{\beta}\right) \stackrel{\epsilon}{\underset{\sim}{\sim}} p_{2 K}^{\alpha \beta \gamma !}\left(E_{\beta}\right), p_{13 K}^{\alpha \beta \gamma !} p_{1 K}^{\alpha \gamma !}\left(E_{\alpha}\right) \stackrel{\epsilon}{\stackrel{\sim}{\sim}} p_{1 K}^{\alpha \beta \gamma !}\left(E_{\alpha}\right) \text {. }
\end{aligned}
$$

Les morphismes $f=\left(f_{\alpha}\right)_{\alpha \in \Lambda}:\left(\left(E_{\alpha}\right)_{\alpha \in \Lambda},\left(\eta_{\alpha \beta}\right)_{\alpha, \beta \in \Lambda}\right) \rightarrow\left(\left(E_{\alpha}^{\prime}\right)_{\alpha \in \Lambda},\left(\eta_{\alpha \beta}^{\prime}\right)_{\alpha, \beta \in \Lambda}\right)$ sont les familles de morphismes $f_{\alpha}: E_{\alpha} \rightarrow E_{\alpha}^{\prime}$ commutant aux données de recollement.

On remarque enfin que, comme $p_{1 K}$ est une immersion ouverte, les foncteurs $p_{1 K}^{\alpha \beta !}$ et $p_{1 K}^{\alpha \beta *}$ sont égaux. De même, pour $p_{2 K}^{\alpha \beta}, p_{12 K}^{\alpha \beta \gamma}, p_{23 K}^{\alpha \beta \gamma}$ et $p_{13 K}^{\alpha \beta \gamma}$. De plus, lorsque $\Lambda$ est réduit à un élément, $\operatorname{Isoc}^{\dagger}(Y, X, \mathcal{P} / K)$ est égale à $\operatorname{Isoc}^{\dagger}(Y, X / K)$.

Proposition 2.5.7. - Il existe un foncteur $\mathcal{L}$ oc $: \operatorname{Isoc}^{\dagger}(Y, X / K) \rightarrow$ $\operatorname{Isoc}^{\dagger}\left(Y, X,\left(\mathcal{P}_{\alpha}\right)_{\alpha \in \Lambda} / K\right)$ canonique et induisant une équivalence de catégorie.

Démonstration. - On associe à chaque $j^{\dagger} \mathcal{O}_{] X[\mathcal{P}}$-module cohérent, $E$, muni d'une connexion intégrable surconvergente, la famille $\left(u_{\alpha K}^{*}\left(\left.E\right|_{]_{X_{\alpha}\left[\mathcal{P}_{\alpha}\right.}}\right)\right)_{\alpha \in \Lambda}$, où 
$u_{\alpha K}^{*}$ est l'image inverse correspondant au diagramme commutatif suivant (voir les notations de 2.3.1) :

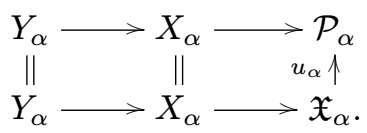

De manière analogue au début de la preuve de 2.5.4 (on remplace $\tau$ par $\epsilon$ ), cette famille est munie d'une structure canonique de donnée de recollement. De même, si $f: E \rightarrow E^{\prime}$ est un morphisme $\operatorname{de} \operatorname{Isoc}^{\dagger}(Y, X / K)$, la famille de morphismes $\left(u_{\alpha K}^{*}\left(\left.f\right|_{]_{X_{\alpha}\left[\mathcal{P}_{\alpha}\right.}}\right)\right)_{\alpha \in \Lambda}$ commute aux données de recollement. On a ainsi construit un foncteur $\mathcal{L} o c: \operatorname{Isoc}^{\dagger}(Y, X / K) \rightarrow \operatorname{Isoc}^{\dagger}\left(Y, X,\left(\mathcal{P}_{\alpha}\right)_{\alpha \in \Lambda} / K\right)$.

Grâce à [3, 2.3.1] (qui implique que les foncteurs $u_{\alpha K}^{*}$ et $u_{\alpha \beta K}^{*}$ sont pleinement fidèles pour les isocristaux surconvergents) on vérifie que ce foncteur est pleinement fidèle.

Prouvons à présent son essentielle surjectivité. Soit $\left(\left(E_{\alpha}\right)_{\alpha \in \Lambda},\left(\eta_{\alpha \beta}\right)_{\alpha, \beta \in \Lambda}\right)$ un objet de $\operatorname{Isoc}^{\dagger}\left(Y, X,\left(\mathcal{P}_{\alpha}\right)_{\alpha \in \Lambda} / K\right)$. D'après [3, 2.3.1], il existe un $j_{\alpha}^{\dagger} \mathcal{O}_{X_{\alpha}\left[\mathcal{P}_{\alpha}\right.}{ }^{-}$ module cohérent $E_{\alpha}^{\prime}$ et un isomorphisme $\iota_{\alpha}: u_{\alpha K}^{*}\left(E_{\alpha}^{\prime}\right) \stackrel{\sim}{\longrightarrow} E_{\alpha}$. Il existe alors un unique isomorphisme $\tau_{\alpha \beta}^{\prime}:\left.\left.E_{\beta}^{\prime}\right|_{X_{\alpha \beta}\left[\mathcal{P}_{\alpha \beta}\right.} \stackrel{\sim}{\longrightarrow} E_{\alpha}^{\prime}\right|_{X_{\alpha \beta}\left[\mathcal{P}_{\alpha \beta}\right.}$ s'inscrivant dans le diagramme commutatif :

$$
\begin{aligned}
& u_{\alpha \beta K}^{*}\left(\left.E_{\beta}^{\prime}\right|_{] X_{\alpha \beta}\left[\mathcal{P}_{\alpha \beta}\right.}\right) \underset{\sim}{\stackrel{\epsilon}{\sim}} p_{2 K}^{\alpha \beta *} u_{\beta K}^{*}\left(E_{\beta}^{\prime}\right) \stackrel{p_{2 K}^{\alpha \beta *}\left(\iota_{\beta}\right)}{\sim} p_{2 K}^{\alpha \beta *}\left(E_{\beta}\right) \\
& u_{\alpha \beta K}^{*}\left(\left.E_{\alpha}^{\prime}\right|_{X_{\alpha \beta}\left[\mathcal{P}_{\alpha \beta}\right.}\right) \stackrel{\epsilon}{\sim} u_{1 K}^{*} p_{\alpha K}^{\alpha \beta *} u_{\alpha K}^{*}\left(E_{\alpha}^{\prime}\right) \stackrel{p_{1 K}^{\alpha \beta *}\left(\iota_{\alpha}\right)}{\sim} p_{1 K}^{\alpha \beta *}\left(E_{\alpha}\right) .
\end{aligned}
$$

Vérifions à présent que les isomorphismes $\tau_{\alpha \beta}^{\prime}$ se recollent. Considérons le diagramme commutatif suivant

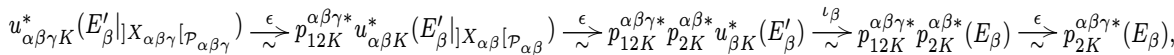

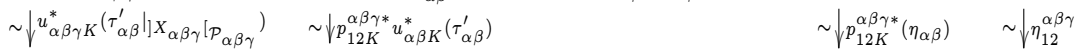

$$
\begin{aligned}
& u_{\alpha \beta \gamma K}^{*}\left(\left.E_{\alpha}^{\prime}\right|_{X_{\alpha \beta \gamma}\left[\mathcal{P}_{\alpha \beta \gamma}\right.}\right) \stackrel{\epsilon}{\underset{\sim}{\sim}} p_{12 K}^{\alpha \beta \gamma *} u_{\alpha \beta K}^{*}\left(\left.E_{\alpha}^{\prime}\right|_{X_{\alpha \beta}\left[\mathcal{P}_{\alpha \beta}\right.}\right) \stackrel{\epsilon}{\sim} p_{12 K}^{\alpha \beta \gamma *} p_{1 K}^{\alpha \beta *} u_{\alpha K}^{*}\left(E_{\alpha}^{\prime}\right) \stackrel{\iota \alpha}{\sim} p_{12 K}^{\alpha \beta \gamma *} p_{1 K}^{\alpha \beta *}\left(E_{\alpha}\right) \stackrel{\epsilon}{\sim} p_{1 K}^{\alpha \beta \gamma *}\left(E_{\alpha}\right),
\end{aligned}
$$

où le rectangle du milieu se déduit de 2.5.7.1 par application du foncteur $p_{12 K}^{\alpha \beta \gamma *}$. On remarque que la flèche composée $d u$ bas de 2.5.7.2 est indépendant du chemin suivi, i.e., le diagramme suivant est commutatif :

$$
\begin{aligned}
& u_{\alpha \beta \gamma K}^{*}\left(\left.E_{\alpha}^{\prime}\right|_{] X_{\alpha \beta \gamma}\left[\mathcal{P}_{\alpha \beta \gamma}\right.}\right) \stackrel{\epsilon}{\sim} p_{12 K}^{\alpha \beta \gamma *} u_{\alpha \beta K}^{*}\left(\left.E_{\alpha}^{\prime}\right|_{X_{\alpha \beta}\left[\mathcal{P}_{\alpha \beta}\right.}\right) \stackrel{\epsilon}{\sim} p_{12 K}^{\alpha \beta \gamma *} p_{1 K}^{\alpha \beta *} u_{\alpha K}^{*}\left(E_{\alpha}^{\prime}\right) \stackrel{\iota_{\alpha}}{\sim} p_{12 K}^{\alpha \beta \gamma *} p_{1 K}^{\alpha \beta *}\left(E_{\alpha}\right) \stackrel{\epsilon}{\sim} p_{1 K}^{\alpha \beta \gamma *}\left(E_{\alpha}\right) \\
& \|\quad \epsilon \uparrow \sim \quad \epsilon \uparrow \sim \quad \epsilon \uparrow \sim \quad\| \\
& u_{\alpha \beta \gamma K}^{*}\left(\left.E_{\alpha}^{\prime}\right|_{] X_{\alpha \beta \gamma}\left[\mathcal{P}_{\alpha \beta \gamma}\right.}\right) \stackrel{\epsilon}{\sim} p_{13 K}^{\alpha \beta \gamma *} u_{\alpha \gamma K}^{*}\left(\left.E_{\alpha}^{\prime}\right|_{X_{\alpha \gamma}\left[\mathcal{P}_{\alpha \gamma}\right.}\right) \stackrel{\epsilon}{\sim} p_{13 K}^{\alpha \beta \gamma *} p_{1 K}^{\alpha \gamma *} u_{\alpha K}^{*}\left(E_{\alpha}^{\prime}\right) \stackrel{\iota_{\alpha}}{\sim} p_{13 K}^{\alpha \beta \gamma *} p_{1 K}^{\alpha \gamma *}\left(E_{\alpha}\right) \stackrel{\epsilon}{\sim} p_{1 K}^{\alpha \beta \gamma *}\left(E_{\alpha}\right) \text {. }
\end{aligned}
$$

De même pour la flèche du haut de 2.5.7.2. En écrivant les deux autres diagrammes analogues à 2.5.7.2, on établit que la famille $\left(E_{\alpha}^{\prime}\right)_{\alpha \in \Lambda}$ se recolle en un 
$j^{\dagger} \mathcal{O}_{] X\left[{ }_{\mathcal{P}}\right.}$-module cohérent $E^{\prime}$, muni d'une connexion intégrable surconvergente. En outre, les isomorphismes $\iota_{\alpha}$ induisent $\operatorname{Loc}\left(E^{\prime}\right) \stackrel{\sim}{\longrightarrow}\left(\left(E_{\alpha}\right)_{\alpha \in \Lambda},\left(\eta_{\alpha \beta}\right)_{\alpha, \beta \in \Lambda}\right)$.

DÉFInition 2.5.8. - Avec les notations de 2.5.7, soient $\left(\left(E_{\alpha}\right)_{\alpha \in \Lambda},\left(\eta_{\alpha \beta}\right)_{\alpha, \beta \in \Lambda}\right)$ un objet de $\operatorname{Isoc}^{\dagger}\left(Y, X,\left(\mathcal{P}_{\alpha}\right)_{\alpha \in \Lambda} / K\right)$ et $E \in \operatorname{Isoc}^{\dagger}(Y, X / K)$. On dira que $\left(\left(E_{\alpha}\right)_{\alpha \in \Lambda},\left(\eta_{\alpha \beta}\right)_{\alpha, \beta \in \Lambda}\right)$ se recolle en $E$, s'il existe un isomorphisme $\mathcal{L} \operatorname{occ}(E) \stackrel{\sim}{\longrightarrow}\left(\left(E_{\alpha}\right)_{\alpha \in \Lambda},\left(\eta_{\alpha \beta}\right)_{\alpha, \beta \in \Lambda}\right)$.

Proposition 2.5.9. - Les foncteurs $\mathrm{sp}_{*}$ et $\mathrm{sp}^{*}$ induisent des équivalences quasi-inverses entre la catégorie $\operatorname{Isoc}^{\dagger}\left(Y, X,\left(\mathcal{P}_{\alpha}\right)_{\alpha \in \Lambda} / K\right)$ et la sous-catégorie pleine de $\operatorname{Coh}\left(X,\left(\mathcal{P}_{\alpha}\right)_{\alpha \in \Lambda}, T\right)$ des objets $\left(\left(\mathcal{E}_{\alpha}\right)_{\alpha \in \Lambda},\left(\theta_{\alpha \beta}\right)_{\alpha, \beta \in \Lambda}\right)$ tels que, pour tout $\alpha \in \Lambda, \mathcal{E}_{\alpha}$ soit $\mathcal{O}_{\mathfrak{X}_{\alpha}, \mathbb{Q}}\left({ }^{\dagger} T \cap X_{\alpha}\right)$-cohérent.

Démonstration. - i) Construisons d'abord le foncteur $\operatorname{sp}_{*}$ : $\operatorname{Isoc}^{\dagger}(Y, X$, $\left.\left(\mathcal{P}_{\alpha}\right)_{\alpha \in \Lambda} / K\right) \rightarrow \operatorname{Coh}\left(X,\left(\mathcal{P}_{\alpha}\right)_{\alpha \in \Lambda}, T\right)$. Si $\left(\left(E_{\alpha}\right)_{\alpha \in \Lambda},\left(\eta_{\alpha \beta}\right)_{\alpha, \beta \in \Lambda}\right) \in \operatorname{Isoc}^{\dagger}(Y, X$, $\left.\left(\mathcal{P}_{\alpha}\right)_{\alpha \in \Lambda} / K\right)$, il suffit de vérifier que $\left(\left(\operatorname{sp}_{*} E_{\alpha}\right)_{\alpha \in \Lambda},\left(\theta_{\alpha \beta}\right)_{\alpha, \beta \in \Lambda}\right)$, où $\theta_{\alpha \beta}$ est l'unique morphisme rendant commutatif le diagramme suivant

$$
\begin{aligned}
& \operatorname{sp}_{*} p_{1 K}^{\alpha \beta !}\left(E_{\alpha}\right) \longrightarrow p_{1}^{\alpha \beta !} \operatorname{sp}_{*}\left(E_{\alpha}\right) \\
& \mathrm{sp}_{*} \eta_{\alpha \beta} \uparrow \sim \theta_{\alpha \beta} \mathrm{A} \\
& \operatorname{sp}_{*} p_{2 K}^{\alpha \beta !}\left(E_{\beta}\right) \longrightarrow p_{2}^{\alpha \beta !} \operatorname{sp}_{*}\left(E_{\beta}\right)
\end{aligned}
$$

dont les isomorphismes horizontaux découlent de 2.4.1, est un objet de $\operatorname{Coh}\left(X,\left(\mathcal{P}_{\alpha}\right)_{\alpha \in \Lambda}, T\right)$, i.e., que les isomorphismes $\theta_{\alpha \beta}$ vérifient la condition de cocycle. Considérons le diagramme commutatif suivant :

$(2.5 .9 .2)$

$$
\begin{aligned}
& \operatorname{sp}_{*} p_{1 K}^{\alpha \beta \gamma !}\left(E_{\alpha}\right) \stackrel{\mathrm{sp}_{*}(\epsilon)}{\sim} \operatorname{sp}_{*} p_{12 K}^{\alpha \beta \gamma *} p_{1 K}^{\alpha \beta *}\left(E_{\alpha}\right) \longrightarrow p_{12}^{\alpha \beta \gamma !} \operatorname{sp}_{*} p_{1 K}^{\alpha \beta *}\left(E_{\alpha}\right) \longrightarrow p_{12}^{\alpha \beta \gamma !} p_{1}^{\alpha \beta !} \operatorname{sp}_{*}\left(E_{\alpha}\right) \stackrel{\tau}{\sim} p_{1}^{\alpha \beta \gamma !}\left(\operatorname{sp}_{*}\left(E_{\alpha}\right)\right) \\
& \operatorname{sp}_{*}\left(\eta_{12}^{\alpha \beta \gamma}\right) \uparrow \sim \quad \operatorname{sp}_{*} p_{12 K}^{\alpha \beta \gamma *}\left(\eta_{\alpha \beta}\right) \uparrow \sim \quad p_{12}^{\alpha \beta \gamma !} \operatorname{sp}_{*}\left(\eta_{\alpha \beta}\right) \uparrow \sim \quad p_{12}^{\alpha \beta \gamma !}\left(\theta_{\alpha \beta}\right) \mid \sim \quad \theta_{12}^{\alpha \beta \gamma} \uparrow \sim \\
& \operatorname{sp}_{*} p_{2 K}^{\alpha \beta \gamma !}\left(E_{\beta}\right) \stackrel{\mathrm{sp}_{*}(\epsilon)}{\sim} \operatorname{sp}_{*} p_{12 K}^{\alpha \beta \gamma *} p_{2 K}^{\alpha \beta *}\left(E_{\beta}\right) \longrightarrow p_{12}^{\alpha \beta \gamma !} \operatorname{sp}_{*} p_{2 K}^{\alpha \beta *}\left(E_{\beta}\right) \longrightarrow p_{12}^{\alpha \beta \gamma !} p_{2}^{\alpha \beta !} \operatorname{sp}_{*}\left(E_{\beta}\right) \stackrel{\tau}{\longrightarrow} p_{2}^{\alpha \beta \gamma !}\left(\operatorname{sp}_{*}\left(E_{\beta}\right)\right) .
\end{aligned}
$$

Or, il découle de 2.4.1 et de 2.4.6 que le morphisme composé du haut (resp. du bas) de 2.5.9.2 est l'isomorphisme canonique $\operatorname{sp}_{*}\left(p_{1 K}^{\alpha \beta \gamma}\right)^{*}\left(E_{\alpha}\right) \stackrel{\sim}{\longrightarrow} p_{1}^{\alpha \beta \gamma !} \operatorname{sp}_{*}\left(E_{\alpha}\right)$ (resp. $\left.\operatorname{sp}_{*}\left(p_{2 K}^{\alpha \beta \gamma}\right)^{*}\left(E_{\beta}\right) \stackrel{\sim}{\longrightarrow} p_{2}^{\alpha \beta \gamma !} \operatorname{sp}_{*}\left(E_{\beta}\right)\right)$. En écrivant les deux autres diagrammes analogues, on conclut que la condition de cocycle est validée.

En outre, si $f=\left(f_{\alpha}\right)_{\alpha \in \Lambda}:\left(\left(E_{\alpha}\right)_{\alpha \in \Lambda},\left(\eta_{\alpha \beta}\right)_{\alpha, \beta \in \Lambda}\right) \rightarrow\left(\left(E_{\alpha}^{\prime}\right)_{\alpha \in \Lambda},\left(\eta_{\alpha \beta}^{\prime}\right)_{\alpha, \beta \in \Lambda}\right)$ est un morphisme de la catégorie $\operatorname{Isoc}^{\dagger}\left(Y, X,\left(\mathcal{P}_{\alpha}\right)_{\alpha \in \Lambda} / K\right)$, on vérifie, par fonctorialité de 2.5.9.1, que la famille $\operatorname{sp}_{*}(f):=\left(\operatorname{sp}_{*}\left(f_{\alpha}\right)\right)_{\alpha \in \Lambda}$ commute aux données de recollement.

ii) Réciproquement, on construit un foncteur, noté sp*, de la manière suivante : soit $\left(\left(\mathcal{E}_{\alpha}\right)_{\alpha \in \Lambda},\left(\theta_{\alpha \beta}\right)_{\alpha, \beta \in \Lambda}\right)$ est un objet de $\operatorname{Coh}\left(X,\left(\mathcal{P}_{\alpha}\right)_{\alpha \in \Lambda}, T\right)$ tel que 
$\mathcal{E}_{\alpha}$ soit $\mathcal{O}_{\mathfrak{X}_{\alpha}, \mathbb{Q}}\left({ }^{\dagger} T \cap X_{\alpha}\right)$-cohérent. En notant $\eta_{\alpha \beta}$ l'unique morphisme rendant commutatif le diagramme suivant

$$
\begin{gathered}
\operatorname{sp}^{*} p_{1}^{\alpha \beta !}\left(\mathcal{E}_{\alpha}\right) \longleftarrow \sim p_{1 K}^{\alpha \beta *} \operatorname{sp}^{*}\left(\mathcal{E}_{\alpha}\right) \\
\operatorname{sp}_{\alpha \beta}^{*} \theta_{\alpha \beta} \uparrow \sim \\
\operatorname{sp}^{*} p_{2}^{\alpha \beta !}\left(\mathcal{E}_{\beta}\right) \longleftarrow \sim p_{2 K}^{\alpha \beta *} \operatorname{sp}^{*}\left(\mathcal{E}_{\beta}\right),
\end{gathered}
$$

on pose $\operatorname{sp}^{*}\left(\left(\mathcal{E}_{\alpha}\right)_{\alpha \in \Lambda},\left(\theta_{\alpha \beta}\right)_{\alpha, \beta \in \Lambda}\right):=\left(\left(\operatorname{sp}^{*} \mathcal{E}_{\alpha}\right)_{\alpha \in \Lambda},\left(\eta_{\alpha \beta}\right)_{\alpha, \beta \in \Lambda}\right)$. De manière analogue à l'étape i) (en utilisant 2.4.1 et 2.4.6), on établit que $\left(\left(\operatorname{sp}^{*} \mathcal{E}_{\alpha}\right)_{\alpha \in \Lambda},\left(\eta_{\alpha \beta}\right)_{\alpha, \beta \in \Lambda}\right) \in \operatorname{Isoc}^{\dagger}\left(Y, X,\left(\mathcal{P}_{\alpha}\right)_{\alpha \in \Lambda} / K\right)$. Enfin, si $f=\left(f_{\alpha}\right)_{\alpha \in \Lambda}$ : $\left(\left(\mathcal{E}_{\alpha}\right)_{\alpha \in \Lambda},\left(\theta_{\alpha \beta}\right)_{\alpha, \beta \in \Lambda}\right) \rightarrow\left(\left(\mathcal{E}_{\alpha}^{\prime}\right)_{\alpha \in \Lambda},\left(\theta_{\alpha \beta}^{\prime}\right)_{\alpha, \beta \in \Lambda}\right)$ est un morphisme, par fonctorialité de 2.5.9.3, la famille $\operatorname{sp}^{*}(f):=\left(\operatorname{sp}^{*}\left(f_{\alpha}\right)\right)_{\alpha \in \Lambda}$ commute aux données de recollement.

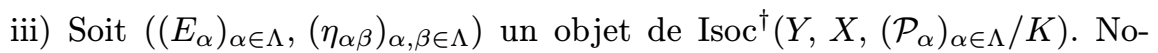
tons $\theta_{\alpha \beta}$ (resp. $\eta_{\alpha \beta}^{\prime}$ ) les isomorphismes de recollement canoniques induits sur $\operatorname{sp}_{*}\left(E_{\alpha}\right)$ (resp. $\operatorname{sp}^{*} \operatorname{sp}_{*}\left(E_{\alpha}\right)$ ). Démontrons maintenant que les isomorphismes d'adjonctions $\operatorname{sp}^{*} \operatorname{sp}_{*}\left(E_{\alpha}\right) \stackrel{\sim}{\longrightarrow} E_{\alpha}$ commutent aux données de recollement, i.e., que le carré de droite du diagramme suivant

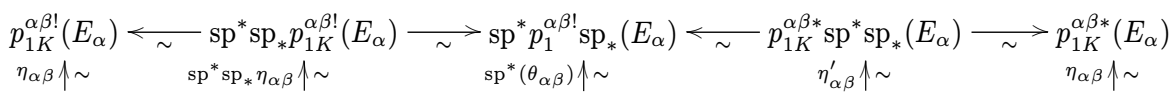

$$
\begin{aligned}
& p_{2 K}^{\alpha \beta !}\left(E_{\beta}\right) \longleftarrow \sim \operatorname{sp}^{*} \operatorname{sp}_{*} p_{2 K}^{\alpha \beta !}\left(E_{\beta}\right) \longrightarrow \operatorname{sp}^{*} p_{2}^{\alpha \beta !} \operatorname{sp}_{*}\left(E_{\beta}\right) \longleftarrow p_{2 K}^{\alpha \beta *} \operatorname{sp}^{*} \operatorname{sp}_{*}\left(E_{\beta}\right) \longrightarrow p_{2 K}^{\alpha \beta *}\left(E_{\beta}\right),
\end{aligned}
$$

est commutatif. La commutativité des deux carrés du milieu découlent respectivement de 2.5.9.1 et 2.5.9.3, tandis que celle du carré de gauche se vérifie par fonctorialité. Or, il dérive de 2.4.1.2 que les morphismes composés du haut et du bas de 2.5.9.4 sont les morphismes identités. Comme toutes les flèches sont des isomorphismes, le diagramme 2.5.9.4 est commutatif.

iv) Soit $\left(\left(\mathcal{E}_{\alpha}\right)_{\alpha \in \Lambda},\left(\theta_{\alpha \beta}\right)_{\alpha, \beta \in \Lambda}\right)$ un objet de $\operatorname{Coh}\left(X,\left(\mathcal{P}_{\alpha}\right)_{\alpha \in \Lambda}, T\right)$ tel que $\mathcal{E}_{\alpha}$ soit $\left.\mathcal{O}_{\mathfrak{X}_{\alpha}, \mathbb{Q}}{ }^{\dagger} T \cap X_{\alpha}\right)$-cohérent. On vérifie de façon similaire à l'étape iii) (on utilise 2.4.2 au lieu de 2.4.1.2), que les isomorphismes d'adjonctions $\mathcal{E}_{\alpha} \stackrel{\sim}{\longrightarrow}$ $\operatorname{sp}_{*} \mathrm{sp}^{*}\left(\mathcal{E}_{\alpha}\right)$ commutent aux données de recollement.

THÉORÈme 2.5.10. - On dispose d'un foncteur canonique pleinement fidèle $\operatorname{sp}_{X \hookrightarrow \mathcal{P}, T+}: \operatorname{Isoc}^{\dagger}(Y, X / K) \rightarrow \operatorname{Coh}(X, \mathcal{P}, T)$. Son image essentielle est constituée par les $\mathcal{D}_{\mathcal{P}}^{\dagger}\left({ }^{\dagger} T\right)_{\mathbb{Q}}$-modules cohérents $\mathcal{E}$ à support dans $X$ satisfaisant l'une des conditions équivalentes suivantes :

(*) pour tout ouvert $\mathcal{P}^{\prime}$ de $\mathcal{P}$ tel que l'immersion fermée $X \cap P^{\prime} \hookrightarrow P^{\prime}$ se relève en un morphisme $v: \mathfrak{X}^{\prime} \hookrightarrow \mathcal{P}^{\prime}$ de $\mathcal{V}$-schémas formels lisses, le faisceau $v^{!}\left(\left.\mathcal{E}\right|_{\mathcal{P}^{\prime}}\right)$ est $\mathcal{O}_{\mathfrak{X}^{\prime}, \mathbb{Q}}\left({ }^{\dagger} T \cap X^{\prime}\right)$-cohérent,

$\left({ }^{* *}\right)$ pour tout ouvert $\mathcal{P}^{\prime}$ affine de $\mathcal{P}$, pour tout relèvement $v: \mathfrak{X}^{\prime} \hookrightarrow \mathcal{P}^{\prime}$ de $X \cap P^{\prime} \hookrightarrow P^{\prime}$, le faisceau $v^{!}\left(\left.\mathcal{E}\right|_{\mathcal{P}^{\prime}}\right)$ est $\mathcal{O}_{\mathfrak{X}^{\prime}, \mathbb{Q}}\left({ }^{\dagger} T \cap X^{\prime}\right)$-cohérent. 
Si $T$ est vide, on le notera $\mathrm{sp}_{X \hookrightarrow \mathcal{P}+}$. Si aucune confusion n'est à craindre, on écrira simplement $\mathrm{sp}_{+}$.

Démonstration. - Il découle de 2.5.7, 2.5.4 et 2.5.9 et avec leurs notations, la construction d'un foncteur canonique pleinement fidèle $\operatorname{sp}_{X \hookrightarrow \mathcal{P}, T+}$ : $\operatorname{Isoc}^{\dagger}(Y, X / K) \rightarrow \operatorname{Coh}(X, \mathcal{P}, T)$ défini en posant $\operatorname{sp}_{X \hookrightarrow \mathcal{P}, T+}:=\mathcal{R}$ ecol $\circ$ $\mathrm{sp}_{*} \circ \mathcal{L}$ oc. À présent, caractérisons son image essentielle. La condition (*) implique $\left.{ }^{* *}\right)$. De plus, soit $\mathcal{E}$ un $\mathcal{D}_{\mathcal{P}}^{\dagger}\left({ }^{\dagger} T\right)_{\mathbb{Q}^{-m o d u l e}}$ cohérent à support dans $X$ satisfaisant la condition $\left({ }^{* *}\right)$. Avec les notations de la preuve de 2.5.4, soit $\mathcal{L} o c(\mathcal{E}):=\left(\left(u_{\alpha}^{!}\left(\left.\mathcal{E}\right|_{\mathcal{P}_{\alpha}}\right)\right)_{\alpha \in \Lambda},\left(\theta_{\alpha \beta}\right)_{\alpha, \beta \in \Lambda}\right)$. Via 2.5 .9 , comme $u_{\alpha}^{!}\left(\left.\mathcal{E}\right|_{\mathcal{P}_{\alpha}}\right)$ est $\mathcal{O}_{\mathfrak{X}_{\alpha}, \mathbb{Q}}\left({ }^{\dagger} T \cap X_{\alpha}\right)$-cohérent, il en résulte que $\mathcal{E}$ est dans l'image essentielle de $\operatorname{sp}_{X \hookrightarrow \mathcal{P}, T+}$. Réciproquement, donnons-nous un objet $\left(\left(\mathcal{E}_{\alpha}\right)_{\alpha \in \Lambda},\left(\theta_{\alpha \beta}\right)_{\alpha, \beta \in \Lambda}\right)$ de $\operatorname{Coh}\left(X,\left(\mathcal{P}_{\alpha}\right)_{\alpha \in \Lambda}, T\right)$ tel que, pour tout $\alpha \in \Lambda, \mathcal{E}_{\alpha}$ soit $\mathcal{O}_{\mathfrak{X}_{\alpha}, \mathbb{Q}}\left({ }^{\dagger} T \cap X_{\alpha}\right)$ cohérent. Posons $\mathcal{E}:=\mathcal{R} \operatorname{ecol}\left(\left(\mathcal{E}_{\alpha}\right)_{\alpha \in \Lambda},\left(\theta_{\alpha \beta}\right)_{\alpha, \beta \in \Lambda}\right)$ et prouvons que $\mathcal{E}$ vérifie la condition $\left(^{*}\right)$. Soit $\mathcal{P}^{\prime}$ un ouvert de $\mathcal{P}$ tel que l'immersion fermée $X \cap P^{\prime} \hookrightarrow P^{\prime}$ se relève en un morphisme $v: \mathfrak{X}^{\prime} \hookrightarrow \mathcal{P}^{\prime}$ de $\mathcal{V}$-schémas formels lisses. Il s'agit de prouver que $v^{!}\left(\left.\mathcal{E}\right|_{\mathcal{P}^{\prime}}\right)$ est $\mathcal{O}_{\mathfrak{X}^{\prime}, \mathbb{Q}}\left({ }^{\dagger} T \cap X^{\prime}\right)$-cohérent, ce qui est local en $\mathfrak{X}^{\prime}$ et $\mathcal{P}^{\prime}$. On peut donc supposer que $\mathfrak{X}^{\prime}$ est affine et qu'il existe $\alpha_{0} \in \Lambda$ tel que $\mathcal{P}^{\prime} \subset \mathcal{P}_{\alpha_{0}}$. Choisissons $\rho_{\alpha}: \mathfrak{X}^{\prime} \hookrightarrow \mathfrak{X}_{\alpha_{0}}$ un relèvement de $X^{\prime} \subset X_{\alpha_{0}}$. On conclut via l'isomorphisme $v^{!}\left(\left.\mathcal{E}\right|_{\mathcal{P}^{\prime}}\right) \stackrel{\tau}{\sim} \underset{\sim}{\sim} \rho_{\alpha}^{!} u_{\alpha}^{!}\left(\left.\mathcal{E}\right|_{\mathcal{P}_{\alpha}}\right)$.

Remarques 2.5.11. - Avec les notations de 2.5.10, grâce à la description de Berthelot des isocristaux surconvergents énoncée dans [12, 2.2.12], $v^{!}\left(\left.\mathcal{E}\right|_{\mathcal{P}^{\prime}}\right)$ est $\mathcal{O}_{\mathfrak{X}^{\prime}, \mathbb{Q}}\left({ }^{\dagger} T \cap X^{\prime}\right)$-cohérent si et seulement si, en notant $\mathcal{Y}^{\prime}$ l'ouvert de $\mathfrak{X}^{\prime}$ complémentaire de $T \cap X^{\prime},\left.v^{!}\left(\left.\mathcal{E}\right|_{\mathcal{P}^{\prime}}\right)\right|_{\mathcal{Y}^{\prime}}$ est $\mathcal{O}_{\mathcal{Y}^{\prime}, \mathbb{Q}^{-}}$-cohérent.

Proposition 2.5.12. - Le foncteur $\operatorname{sp}_{X \hookrightarrow \mathcal{P}, T+}$ ne dépend que de $X \hookrightarrow \mathcal{P}$ et de $T$.

Démonstration. - Il s'agit de prouver que le foncteur $\operatorname{sp}_{X \hookrightarrow \mathcal{P}, T+}$ est indépendant du choix des recouvrements ouverts de $P$, ni de celui des relèvements qu'il induit. Donnons-nous un deuxième recouvrement d'ouverts affines $\mathcal{P}=\cup_{\alpha^{\prime} \in \Lambda^{\prime}} \mathcal{P}_{\alpha^{\prime}}$. En remplaçant respectivement $\alpha, \beta, \gamma \in \Lambda$ par $\alpha^{\prime}, \beta^{\prime}, \gamma^{\prime} \in \Lambda^{\prime}$, on prend pour ce recouvrement les notations analogues à 2.5.1.

Dans un premier temps, supposons qu'il existe une application surjective $\rho$ : $\Lambda^{\prime} \rightarrow \Lambda$ telle que, $\mathcal{P}_{\alpha^{\prime}} \subset \mathcal{P}_{\rho\left(\alpha^{\prime}\right)}$ et $\mathcal{P}_{\alpha}=\cup_{\alpha^{\prime} \in \rho^{-1}(\alpha)} \mathcal{P}_{\alpha^{\prime}}$. Pour tout $\left(\alpha^{\prime}, \beta^{\prime}, \gamma^{\prime}\right) \in$ $\Lambda^{\prime 3}$, on choisit alors des relèvements $\epsilon_{\alpha^{\prime}}: \mathfrak{X}_{\alpha^{\prime}} \rightarrow \mathfrak{X}_{\rho\left(\alpha^{\prime}\right)}, \epsilon_{\alpha^{\prime} \beta^{\prime}}: \mathfrak{X}_{\alpha^{\prime} \beta^{\prime}} \rightarrow$ $\mathfrak{X}_{\rho\left(\alpha^{\prime}\right) \rho\left(\beta^{\prime}\right)}$ et $\epsilon_{\alpha^{\prime} \beta^{\prime} \gamma^{\prime}}: \mathfrak{X}_{\alpha^{\prime} \beta^{\prime} \gamma^{\prime}} \rightarrow \mathfrak{X}_{\rho\left(\alpha^{\prime}\right) \rho\left(\beta^{\prime}\right) \rho\left(\gamma^{\prime}\right)}$. On construit alors un foncteur

$$
\begin{aligned}
& \operatorname{Loc}_{\left(\mathcal{P}_{\alpha^{\prime}}\right)_{\alpha^{\prime} \in \Lambda^{\prime}},\left(\mathcal{P}_{\alpha}\right)_{\alpha \in \Lambda}}: \operatorname{Coh}\left(X,\left(\mathcal{P}_{\alpha}\right)_{\alpha \in \Lambda}, T\right) \rightarrow \operatorname{Coh}\left(X,\left(\mathcal{P}_{\alpha^{\prime}}\right)_{\alpha^{\prime} \in \Lambda^{\prime}}, T\right) \\
& \text { томе } 137-2009-\mathrm{N}^{\circ} 4
\end{aligned}
$$


de la façon suivante : pour tout objet $\left(\left(\mathcal{E}_{\alpha}\right)_{\alpha \in \Lambda},\left(\theta_{\alpha \beta}\right)_{\alpha, \beta \in \Lambda}\right)$ de $\operatorname{Coh}(X$, $\left.\left(\mathcal{P}_{\alpha}\right)_{\alpha \in \Lambda}, T\right)$, on définit $\operatorname{L}_{\left.o c_{\left(\mathcal{P}^{\prime}\right.}\right)_{\alpha^{\prime} \in \Lambda^{\prime}},\left(\mathcal{P}_{\alpha}\right)_{\alpha \in \Lambda}}\left(\left(\mathcal{E}_{\alpha}\right)_{\alpha \in \Lambda},\left(\theta_{\alpha \beta}\right)_{\alpha, \beta \in \Lambda}\right)=$ $\left(\left(\mathcal{E}_{\alpha^{\prime}}\right)_{\alpha^{\prime} \in \Lambda^{\prime}},\left(\theta_{\alpha^{\prime} \beta^{\prime}}\right)_{\alpha^{\prime}, \beta^{\prime} \in \Lambda^{\prime}}\right)$, où $\mathcal{E}_{\alpha^{\prime}}:=\epsilon_{\alpha^{\prime}}^{!}\left(\mathcal{E}_{\rho\left(\alpha^{\prime}\right)}\right)$ et $\theta_{\alpha^{\prime} \beta^{\prime}}$ est l'unique morphisme induisant le diagramme commutatif :

$$
\begin{aligned}
& p_{1}^{\alpha^{\prime} \beta^{\prime} !} \epsilon_{\alpha^{\prime}}^{!}\left(\mathcal{E}_{\rho\left(\alpha^{\prime}\right)}\right) \stackrel{\tau}{\sim} \underset{\theta_{\alpha^{\prime} \beta^{\prime}} \wedge}{\sim} \epsilon_{\alpha^{\prime} \beta^{\prime}}^{!} p_{1}^{\rho\left(\alpha^{\prime}\right) \rho\left(\beta^{\prime}\right) !}\left(\mathcal{E}_{\rho\left(\alpha^{\prime}\right)}\right) \\
& p_{2}^{\alpha^{\prime} \beta^{\prime} !} \epsilon_{\alpha^{\prime} \beta^{\prime}}^{!}\left(\theta_{\rho\left(\alpha^{\prime}\right) \rho\left(\beta^{\prime}\right) \uparrow \sim}\left(\mathcal{E}_{\rho\left(\beta^{\prime}\right)}\right) \stackrel{\tau}{\sim} \underset{\sim}{\sim} \epsilon_{\alpha^{\prime} \beta^{\prime}}^{!} p_{2}^{\rho\left(\alpha^{\prime}\right) \rho\left(\beta^{\prime}\right) !}\left(\mathcal{E}_{\rho\left(\beta^{\prime}\right)}\right) .\right.
\end{aligned}
$$

On vérifie que les isomorphismes $\theta_{\alpha^{\prime} \beta^{\prime}}$ satisfont à la condition de cocycle. Enfin, en transformant le carré 2.5.12.1 en cube, on obtient la fonctorialité de $\mathcal{L} c_{\left(\mathcal{P}_{\alpha^{\prime}}\right)_{\alpha^{\prime} \in \Lambda^{\prime}},\left(\mathcal{P}_{\alpha}\right)_{\alpha \in \Lambda}}$.

De manière analogue (en remplaçant $\mathcal{E}$ par $E, \theta$ par $\eta$ et $\tau$ par $\epsilon$ ), on construit un foncteur

$\operatorname{Loc}_{\left(\mathcal{P}_{\alpha^{\prime}}\right)_{\alpha^{\prime} \in \Lambda^{\prime}},\left(\mathcal{P}_{\alpha}\right)_{\alpha \in \Lambda}}: \operatorname{Isoc}^{\dagger}\left(Y, X,\left(\mathcal{P}_{\alpha}\right)_{\alpha \in \Lambda} / K\right) \rightarrow \operatorname{Isoc}^{\dagger}\left(Y, X,\left(\mathcal{P}_{\alpha^{\prime}}\right)_{\alpha^{\prime} \in \Lambda^{\prime}} / K\right)$.

Pour ces deux foncteurs, on dispose de l'isomorphisme $\mathcal{L}_{o c}\left(\mathcal{P}_{\alpha^{\prime}}\right)_{\alpha^{\prime} \in \Lambda^{\prime}},\left(\mathcal{P}_{\alpha}\right)_{\alpha \in \Lambda} \circ$ $\mathcal{L} o c \stackrel{\sim}{\sim} \mathcal{L}$ oc. Il reste alors à prouver la commutation suivante : $\mathrm{sp}^{*} \circ$

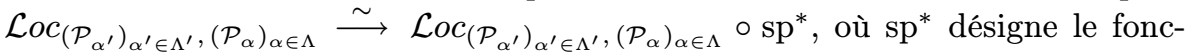
teur de 2.5.9. Soit $\left(\left(\mathcal{E}_{\alpha}\right)_{\alpha \in \Lambda},\left(\theta_{\alpha \beta}\right)_{\alpha, \beta \in \Lambda}\right)$ un objet de $\operatorname{Coh}\left(X,\left(\mathcal{P}_{\alpha}\right)_{\alpha \in \Lambda}, T\right)$ tel que, pour tout $\alpha \in \Lambda, \mathcal{E}_{\alpha}$ soit $\mathcal{O}_{\mathfrak{X}_{\alpha}, \mathbb{Q}}\left({ }^{\dagger} T \cap X_{\alpha}\right)$-cohérent. Avec les notations analogues à 2.5.9.3, considérons le diagramme

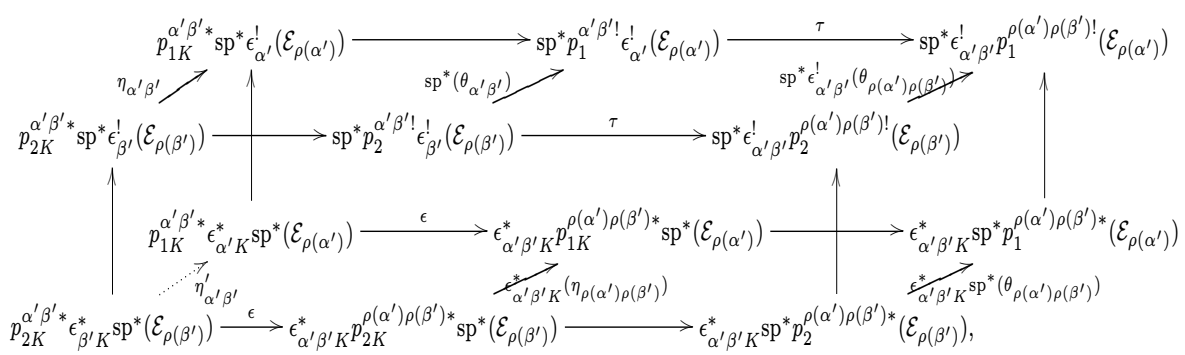

où les carrés horizontaux sont commutatifs par définition. Il découle de 2.4.1 et de 2.4.6 que les rectangles de devant et de derrière le sont aussi. Comme il en est de même par fonctorialité du carré de droite, il en dérive que le carré de gauche est commutatif, i.e., que l'isomorphisme canonique $\operatorname{sp}^{*} \epsilon_{\alpha^{\prime}}^{!}\left(\mathcal{E}_{\rho\left(\alpha^{\prime}\right)}\right) \stackrel{\sim}{\longrightarrow}$ $\epsilon_{\alpha^{\prime} K^{\prime}}^{*} \mathrm{sp}^{*}\left(\mathcal{E}_{\rho\left(\alpha^{\prime}\right)}\right)$ commute aux données de recollement respectives.

Passons à présent au cas général. En utilisant le troisième recouvrement $\mathcal{P}=\cup_{\left(\alpha, \alpha^{\prime}\right) \in \Lambda \times \Lambda^{\prime}} \mathcal{P}_{\alpha} \cap \mathcal{P}_{\alpha^{\prime}}$, on se ramène au premier cas. 
3. Comparaison des foncteurs duaux des isocristaux surconvergents : compatibilité aux images inverses (extraordinaires) par une immersion ouverte

On désignera par $f: \mathfrak{X}^{\prime} \rightarrow \mathfrak{X}$ une immersion ouverte de $\mathcal{V}$-schémas formels lisses, $T$ un diviseur de $X$ et $T^{\prime}:=f^{-1}(T)$ le diviseur de $X^{\prime}$ correspondant. On note $\mathcal{Y}$ l'ouvert de $\mathfrak{X}$ complémentaire de $T$ et on désigne par $E$ un isocristal sur $Y$ surconvergent le long de $T$. Le dual de $E$ sera noté $E^{\vee}$. De même pour tout $\mathcal{O}_{\mathfrak{X}}\left({ }^{\dagger} T\right)_{\mathbb{Q}^{-}}$module $\mathcal{E}, \mathcal{E}^{\vee}$ sera son dual $\mathcal{O}_{\mathfrak{X}}\left({ }^{\dagger} T\right)_{\mathbb{Q}}$-linéaire.

Nous avons construit dans [10, 2.2.12], l'isomorphisme canonique de commutation aux foncteurs duaux suivant $: \operatorname{sp}_{*}\left(E^{\vee}\right) \stackrel{\sim}{\longrightarrow} \mathbb{D}_{T}^{\dagger} \operatorname{sp}_{*}(E)$. Nous vérifions dans cette section que celui-ci commute aux images inverses (extraordinaires) respectives par $f$, i.e., que le diagramme 3.3.13.1 est commutatif. Notons que lorsque $f$ n'est pas une immersion ouverte, le foncteur dual $\mathbb{D}_{T}^{\dagger}$ ne commute pas a priori au foncteur $f^{!}$(mais lorsque $f$ est lisse, à un décalage près, cela reste conjecturalement vrai).

Comme le cas de la restriction à un ouvert est immédiat, sauf pour 3.3.13, on supposera pour simplifier que $f$ est un isomorphisme.

\subsection{Images directes et images inverses extraordinaires dans le cas d'un isomorphisme}

3.1.1. - On note $\mathcal{D}_{\mathfrak{X}}\left({ }^{\dagger} T\right)_{\mathbb{Q}}:=\mathcal{O}_{\mathfrak{X}}\left({ }^{\dagger} T\right)_{\mathbb{Q}} \otimes_{\mathcal{O}_{\mathfrak{X}, \mathbb{Q}}} \mathcal{D}_{\mathfrak{X}, \mathbb{Q}}$ (de même avec des primes). Le morphisme $\mathcal{D}_{\mathfrak{X}^{\prime}}\left({ }^{\dagger} T^{\prime}\right)_{\mathbb{Q}}$-linéaire à gauche canonique $\mathcal{D}_{\mathfrak{X}^{\prime}}\left({ }^{\dagger} T^{\prime}\right)_{\mathbb{Q}} \rightarrow$ $f^{*} \mathcal{D}_{\mathfrak{X}}\left({ }^{\dagger} T\right)_{\mathbb{Q}}(\operatorname{voir}[5,2.1 .3 .1])$ est un isomorphisme. On note $\iota$, l'isomorphisme composé : $f^{-1} \mathcal{D}_{\mathfrak{X}}\left({ }^{\dagger} T\right)_{\mathbb{Q}} \stackrel{\sim}{\longrightarrow} f^{*} \mathcal{D}_{\mathfrak{X}}\left({ }^{\dagger} T\right)_{\mathbb{Q}} \stackrel{\sim}{\sim} \mathcal{D}_{\mathfrak{X}^{\prime}}\left({ }^{\dagger} T^{\prime}\right)_{\mathbb{Q}}$.

Proposition 3.1.2. - L'isomorphisme $\iota: f^{-1} \mathcal{D}_{\mathfrak{X}}\left({ }^{\dagger} T\right)_{\mathbb{Q}} \stackrel{\sim}{\longrightarrow} \mathcal{D}_{\mathfrak{X}^{\prime}}\left({ }^{\dagger} T^{\prime}\right)_{\mathbb{Q}}$ est un isomorphisme d'anneaux.

Démonstration. - Pour tout entier $n$, on écrit $\mathcal{P}_{\mathfrak{X}, n}:=\mathcal{O}_{\mathfrak{X} \times \mathfrak{X}} / \mathcal{I}^{n+1}$, où $\mathcal{I}$ désigne l'idéal de l'immersion diagonale de $\mathfrak{X}$. On posera $\widetilde{\mathcal{P}}_{\mathfrak{X}, n}:=\mathcal{O}_{\mathfrak{X}}\left({ }^{\dagger} T\right)_{\mathbb{Q}} \otimes_{\mathcal{O}_{\mathfrak{X}}}$ $\mathcal{P}_{\mathfrak{X}, n}$ et $\widetilde{\mathcal{D}}_{\mathfrak{X}, n}$ son dual $\mathcal{O}_{\mathfrak{X}}\left({ }^{\dagger} T\right)_{\mathbb{Q}}$-linéaire pour la structure gauche (de même en rajoutant des primes).

On remarque d'abord que l'isomorphisme $\iota$ envoie $f^{-1} \widetilde{\mathcal{D}}_{\mathfrak{X}, n}$ sur $\widetilde{\mathcal{D}}_{\mathfrak{X}^{\prime}, n}$. En effet, cela dérive du diagramme commutatif :

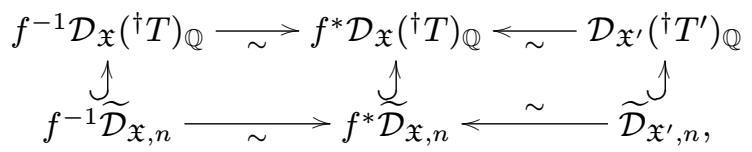

dont la flèche en bas à droite découle par dualité du morphisme canonique $f^{*} \widetilde{\mathcal{P}}_{\mathfrak{X}, n} \rightarrow \widetilde{\mathcal{P}}_{\mathfrak{X}^{\prime}, n}$. 
Soient $n, n^{\prime}$ deux entiers, $P \in f^{-1} \widetilde{\mathcal{D}}_{\mathfrak{X}, n}, Q \in f^{-1} \widetilde{\mathcal{D}}_{\mathfrak{X}, n^{\prime}}$. En notant $1 \otimes P: f^{*} \widetilde{\mathcal{P}}_{\mathfrak{X}, n} \rightarrow \mathcal{O}_{\mathfrak{X}^{\prime}}\left({ }^{\dagger} T^{\prime}\right)_{\mathbb{Q}}$ l'image de $P$ par $f^{-1} \widetilde{\mathcal{D}}_{\mathfrak{X}, n} \stackrel{\sim}{\longrightarrow} f^{*} \widetilde{\mathcal{D}}_{\mathfrak{X}, n} \stackrel{\sim}{\longrightarrow}$ $\mathcal{H o m}_{\mathcal{O}_{\mathfrak{X}^{\prime}}\left({ }^{\dagger} T^{\prime}\right)_{\mathbb{Q}}}\left(f^{*} \widetilde{\mathcal{P}}_{\mathfrak{X}, n}, \mathcal{O}_{\mathfrak{X}^{\prime}}\left({ }^{\dagger} T^{\prime}\right)_{\mathbb{Q}}\right)$, via 3.1.2.1, on obtient tautologiquement le diagramme commutatif :

$$
\begin{aligned}
\widetilde{\mathcal{P}}_{\mathfrak{X}^{\prime}, n} \underset{\sim}{\longleftarrow} f^{*} \widetilde{\mathcal{P}}_{\mathfrak{X}, n} \longleftarrow & \sim f^{-1} \widetilde{\mathcal{P}}_{\mathfrak{X}, n} \\
& \downarrow P \\
\left.\iota^{\prime(P)}\right) & \left.\mathcal{O}_{\mathfrak{X}^{\prime}}{ }^{\dagger} T^{\prime}\right)_{\mathbb{Q}} \longleftarrow \sim f^{-1} \mathcal{O}_{\mathfrak{X}}\left({ }^{\dagger} T\right)_{\mathbb{Q}} .
\end{aligned}
$$

Considérons maintenant le diagramme suivant :

$$
\begin{gathered}
f^{-1} \widetilde{\mathcal{P}}_{\mathfrak{X}, n+n^{\prime}} \stackrel{\widetilde{\delta}^{n, n^{\prime}}}{\longrightarrow} f^{-1} \widetilde{\mathcal{P}}_{\mathfrak{X}, n} \otimes_{f^{-1} \mathcal{O}_{\mathfrak{X}}(\dagger T)_{\mathbb{Q}}} f^{-1} \widetilde{\mathcal{P}}_{\mathfrak{X}, n^{\prime}} \stackrel{Q}{\longrightarrow} f^{-1} \widetilde{\mathcal{P}}_{\mathfrak{X}, n} \stackrel{P}{\longrightarrow} f^{-1} \mathcal{O}_{\mathfrak{X}}\left({ }^{\dagger} T\right)_{\mathbb{Q}} \\
\widetilde{\mathcal{P}}_{\mathfrak{X}^{\prime}, n+n^{\prime}} \stackrel{\widetilde{\delta}^{n, n^{\prime}}}{\longrightarrow} \widetilde{\mathcal{P}}_{\mathfrak{X}^{\prime}, n} \otimes_{\left.\mathcal{O}_{\mathfrak{X}^{\prime}}{ }^{\dagger} T^{\prime}\right)_{\mathbb{Q}}} \widetilde{\mathcal{P}}_{\mathfrak{X}^{\prime}, n^{\prime}} \stackrel{\iota(Q)}{\longrightarrow} \widetilde{\mathcal{P}}_{\mathfrak{X}^{\prime}, n} \stackrel{\iota(P)}{\longrightarrow} \mathcal{O}_{\mathfrak{X}^{\prime}}\left({ }^{\dagger} T^{\prime}\right)_{\mathbb{Q}},
\end{gathered}
$$

dont les isomorphismes $\widetilde{\delta}^{n, n^{\prime}}$ sont ceux analogues à [10, 1.1.10]. Par définition, le composé du haut (resp. du bas) donne $P \cdot Q($ resp. $\iota(P) \cdot \iota(Q))$. Ainsi, par 3.1.2.2, il s'agit d'établir la commutativité de 3.1.2.3. On déduit de 3.1.2.2 la commutativité des carrés du milieu et de droite. Quant à celle du carré de gauche, cela dérive d'un calcul immédiat (on utilise la caractérisation de $\widetilde{\delta}^{n, n^{\prime}}$ : $\left.\widetilde{\delta}^{n, n^{\prime}}(a \otimes b)=(a \otimes 1) \otimes(1 \otimes b)\right)$. D'où le résultat.

\subsection{3. - On remarque que le diagramme}

$$
\begin{gathered}
\left.f^{-1} \mathcal{O}_{\mathfrak{X}}\left({ }^{\dagger} T\right)_{\mathbb{Q}} \underset{\mathcal{X}^{\prime}}{ } \stackrel{\sim}{\sim} \mathcal{O}^{\dagger} T^{\prime}\right)_{\mathbb{Q}} \\
\downarrow \\
f^{-1} \mathcal{D}_{\mathfrak{X}}\left({ }^{\dagger} T\right)_{\mathbb{Q}} \stackrel{\iota}{\sim} \mathcal{D}_{\mathfrak{X}^{\prime}}\left({ }^{\dagger} T^{\prime}\right)_{\mathbb{Q}}
\end{gathered}
$$

est commutatif. On dispose ainsi, pour tout $\mathcal{E} \in D_{\text {coh }}^{\mathrm{b}}\left({ }^{\mathrm{g}} \mathcal{D}_{\mathfrak{X}}\left({ }^{\dagger} T\right)_{\mathbb{Q}}\right)$, de l'isomorphisme canonique $f^{*}(\mathcal{E})=\mathcal{O}_{\mathfrak{X}^{\prime}}\left({ }^{\dagger} T^{\prime}\right)_{\mathbb{Q}} \otimes_{f^{-1}} \mathcal{O}_{\mathfrak{X}}\left({ }^{\dagger} T\right)_{\mathbb{Q}} \mathcal{E} \stackrel{\sim}{\longrightarrow} \mathcal{D}_{\mathfrak{X}^{\prime}}\left({ }^{\dagger} T^{\prime}\right)_{\mathbb{Q}} \otimes_{f^{-1}} \mathcal{D}_{\mathfrak{X}}\left({ }^{\dagger} T\right)_{\mathbb{Q}}$ $\mathcal{E}$. De même en remplaçant $\mathcal{D}$ par $\mathcal{D}^{\dagger}$.

En voyant $\mathcal{D}_{\mathfrak{X}^{\prime}}\left({ }^{\dagger} T^{\prime}\right)_{\mathbb{Q}}$ comme un $\left(\mathcal{D}_{\mathfrak{X}^{\prime}}\left({ }^{\dagger} T^{\prime}\right)_{\mathbb{Q}}, f^{-1} \mathcal{D}_{\mathfrak{X}}\left({ }^{\dagger} T\right)_{\mathbb{Q}}\right)$-bimodule, on obtient l'isomorphisme canonique $\mathcal{D}_{\mathfrak{X}^{\prime}}\left({ }^{\dagger} T^{\prime}\right)_{\mathbb{Q}} \stackrel{\sim}{\longrightarrow} \mathcal{D}_{\mathfrak{X}^{\prime} \rightarrow \mathfrak{X}}\left({ }^{\dagger} T\right)_{\mathbb{Q}}$ de $\left(\mathcal{D}_{\mathfrak{X}^{\prime}}\left({ }^{\dagger} T^{\prime}\right)_{\mathbb{Q}}, f^{-1} \mathcal{D}_{\mathfrak{X}}\left({ }^{\dagger} T\right)_{\mathbb{Q}}\right)$-bimodules. Dans la suite, on pourra identifier canoniquement $\mathcal{D}_{\mathfrak{X}^{\prime} \rightarrow \mathfrak{X}}\left({ }^{\dagger} T\right)_{\mathbb{Q}}$ et $\mathcal{D}_{\mathfrak{X}^{\prime}}\left({ }^{\dagger} T^{\prime}\right)_{\mathbb{Q}}$.

\section{Proposition 3.1.4. - Les isomorphismes canoniques}

$$
\begin{aligned}
& f^{-1} \omega_{\mathfrak{X}} \otimes_{f^{-1}} \mathcal{O}_{\mathfrak{X}} \mathcal{D}_{\mathfrak{X}^{\prime}}\left({ }^{\dagger} T^{\prime}\right)_{\mathbb{Q}} \stackrel{\sim}{\longrightarrow} \omega_{\mathfrak{X}^{\prime}} \otimes_{\mathcal{O}_{\mathfrak{X}^{\prime}}} \mathcal{D}_{\mathfrak{X}^{\prime}}\left({ }^{\dagger} T^{\prime}\right)_{\mathbb{Q}}, \\
& \mathcal{D}_{\mathfrak{X}^{\prime}}\left({ }^{\dagger} T^{\prime}\right)_{\mathbb{Q}} \otimes_{f^{-1} \mathcal{O}_{\mathfrak{X}}} f^{-1} \omega_{\mathfrak{X}}^{-1} \stackrel{\sim}{\longrightarrow} \mathcal{D}_{\mathfrak{X}^{\prime}}\left({ }^{\dagger} T^{\prime}\right)_{\mathbb{Q}} \otimes_{\mathcal{O}_{\mathfrak{X}^{\prime}}} \omega_{\mathfrak{X}^{\prime}}^{-1}, \\
&\left.\omega_{\mathfrak{X}^{\prime}} \otimes_{\mathcal{O}_{\mathfrak{X}^{\prime}}} \mathcal{D}_{\mathfrak{X}^{\prime}}\left({ }^{\dagger} T^{\prime}\right)_{\mathbb{Q}} \otimes_{\mathcal{O}_{\mathfrak{X}^{\prime}}} \omega_{\mathfrak{X}^{\prime}}^{-1} \stackrel{\sim}{\longrightarrow} \mathcal{D}_{\mathfrak{X} \leftarrow \mathfrak{X}^{\prime}}{ }^{\dagger} T\right)_{\mathbb{Q}},
\end{aligned}
$$


sont $f^{-1} \mathcal{D}_{\mathfrak{X}}\left({ }^{\dagger} T\right)_{\mathbb{Q}}$-bilinéaires pour les deux premiers et $\left(f^{-1} \mathcal{D}_{\mathfrak{X}}\left({ }^{\dagger} T\right)_{\mathbb{Q}}, \mathcal{D}_{\mathfrak{X}^{\prime}}\left({ }^{\dagger} T^{\prime}\right)_{\mathbb{Q}}\right)$-bilinéaire pour le dernier.

Démonstration. - La proposition a bien un sens. En effet, en considérant le faisceau $\mathcal{D}_{\mathfrak{X}^{\prime}}\left({ }^{\dagger} T^{\prime}\right)_{\mathbb{Q}}$ comme un $\left(f^{-1} \mathcal{D}_{\mathfrak{X}}\left({ }^{\dagger} T\right)_{\mathbb{Q}}, \mathcal{D}_{\mathfrak{X}^{\prime}}\left({ }^{\dagger} T^{\prime}\right)_{\mathbb{Q}}\right)$-bimodule, $f^{-1} \omega_{\mathfrak{X}} \otimes_{f^{-1} \mathcal{O}_{\mathfrak{X}}} \mathcal{D}_{\mathfrak{X}^{\prime}}\left({ }^{\dagger} T^{\prime}\right)_{\mathbb{Q}}$ devient un $\left(f^{-1} \mathcal{D}_{\mathfrak{X}}\left({ }^{\dagger} T\right)_{\mathbb{Q}}, \mathcal{D}_{\mathfrak{X}^{\prime}}\left({ }^{\dagger} T^{\prime}\right)_{\mathbb{Q}}\right)$-bimodule à droite et donc, grâce à $\iota$, un $\mathcal{D}_{\mathfrak{X}^{\prime}}\left({ }^{\dagger} T^{\prime}\right)_{\mathbb{Q}}$-bimodule à droite. De même, pour les deux autres isomorphismes 3.1.4.2 et 3.1.4.3.

La proposition est locale. Supposons donc $\mathfrak{X}$ affine muni de coordonnées locales $t_{1}, \ldots, t_{d}$. Notons $t_{1}^{\prime}, \ldots, t_{d}^{\prime}$ les coordonnées locales sur $\mathfrak{X}^{\prime}$ correspondantes, $\partial_{1}, \ldots, \partial_{d}$ et $\partial_{1}^{\prime}, \ldots, \partial_{d}^{\prime}$ les dérivations associées. On peut alors identifer $\omega_{\mathfrak{X}}$ à $\mathcal{O}_{\mathfrak{X}}$ grâce à la base $d t_{1} \wedge \cdots \wedge d t_{d}$, et $\omega_{\mathfrak{X}}^{-1}$ à $\mathcal{O}_{\mathfrak{X}}$ grâce à la base duale. De même en remplaçant $\mathfrak{X}$ par $\mathfrak{X}^{\prime}$ et $t$ par $t^{\prime}$. Ces identifications sont compatibles car l'image de $d t_{1} \wedge \cdots \wedge d t_{d}$ par l'isomorphisme canonique $f^{-1} \omega_{\mathfrak{X}} \otimes_{f-1} \mathcal{O}_{\mathfrak{X}} \mathcal{O}_{\mathfrak{X}^{\prime}} \stackrel{\sim}{\longrightarrow} \omega_{\mathfrak{X}^{\prime}}$ est $d t_{1}^{\prime} \wedge \cdots \wedge d t_{d}^{\prime}$ (de même en passant au dual).

Avec ces identifications, l'action par la structure tordue se calcule avec l'adjoint (voir [5, 1.2.2.1]). En effet, cela découle de la formule essentielle [5, $1.2 .3]$.

La flèche $\iota$ envoie $\underline{\partial}^{[k]}:=\partial_{1}^{k_{1}} \cdots \partial_{d}^{k_{d}}$ sur $\underline{\partial}^{\prime[k]}:=\partial_{1}^{\prime k_{1}} \cdots \partial_{d}^{\prime k_{d}}$ et $\sum_{\underline{k}} a_{\underline{k}} \underline{\partial}^{[k]}$ sur $\sum_{\underline{k}}\left(1 \otimes a_{\underline{k}}\right) \underline{\partial}^{\prime}{ }^{[k]}$, où $1 \otimes a_{\underline{k}}$ est l'image canonique de $a_{\underline{k}} \in f^{-1} \mathcal{O}_{\mathfrak{X}}\left(^{\dagger} T\right)_{\mathbb{Q}}$ dans $\mathcal{O}_{\mathfrak{X}^{\prime}}\left({ }^{\dagger} T^{\prime}\right)_{\mathbb{Q}}$. Puisque $\iota$ est un homomorphisme d'anneaux, on calcule que pour tout $P \in f^{-1} \mathcal{D}_{\mathfrak{X}}\left({ }^{\dagger} T\right)_{\mathbb{Q}},{ }^{\mathrm{t}} \iota(P)=\iota\left({ }^{\mathrm{t}} P\right)$, où $\ll \mathrm{t}$ » désigne l'adjoint.

3.1.5. - De manière analogue à 3.1.2, on prouve que l'isomorphisme composé $f^{-1} \mathcal{D}_{X_{i}}^{(m)}(T) \stackrel{\sim}{\longrightarrow} f^{*} \mathcal{D}_{X_{i}}^{(m)}(T) \stackrel{\sim}{\longleftarrow} \mathcal{D}_{X_{i}^{\prime}}^{(m)}\left(T^{\prime}\right)$ est un isomorphisme d'anneaux. Il en résulte, avec les notations de [12, 1.1.2], par complétion et passage à la limite sur le niveau, que $\left.f^{-1} \mathcal{D}_{\mathfrak{X}}^{\dagger}\left({ }^{\dagger} T\right)_{\mathbb{Q}} \stackrel{\sim}{\longrightarrow} \mathcal{D}_{\mathfrak{X}^{\prime} \rightarrow \mathfrak{X}}^{\dagger}{ }^{\dagger} T\right)_{\mathbb{Q}} \stackrel{\sim}{\sim} \mathcal{D}_{\mathfrak{X}^{\prime}}^{\dagger}\left({ }^{\dagger} T^{\prime}\right)_{\mathbb{Q}}$ est un isomorphisme d'anneaux. Les isomorphismes de 3.1.4.1 sont encore valables en remplaçant $\mathcal{D}$ par $\mathcal{D}^{\dagger}$. Ainsi, l'isomorphisme canonique $\omega_{\mathfrak{X}^{\prime}} \otimes_{\mathcal{O}_{\mathfrak{X}^{\prime}}}$ $\mathcal{D}_{\mathfrak{X}^{\prime}}^{\dagger}\left({ }^{\dagger} T^{\prime}\right)_{\mathbb{Q}} \otimes_{\mathcal{O}_{\mathfrak{X}^{\prime}}} \omega_{\mathfrak{X}^{\prime}}^{-1} \stackrel{\sim}{\longrightarrow} \mathcal{D}_{\mathfrak{X} \leftarrow \mathfrak{X}^{\prime}}^{\dagger}\left({ }^{\dagger} T\right)_{\mathbb{Q}}$ est $\left(f^{-1} \mathcal{D}_{\mathfrak{X}}^{\dagger}\left({ }^{\dagger} T\right)_{\mathbb{Q}}, \mathcal{D}_{\mathfrak{X}^{\prime}}^{\dagger}\left({ }^{\dagger} T^{\prime}\right)_{\mathbb{Q}}\right)$-bilinéaire. Par la suite, on identifiera $\mathcal{D}_{\mathfrak{X}^{\prime} \rightarrow \mathfrak{X}}^{\dagger}\left({ }^{\dagger} T\right)_{\mathbb{Q}}$ à $\mathcal{D}_{\mathfrak{X}^{\prime}}^{\dagger}\left({ }^{\dagger} T^{\prime}\right)_{\mathbb{Q}}$ et $\left.\mathcal{D}_{\mathfrak{X} \leftarrow \mathfrak{X}^{\prime}}^{\dagger}{ }^{\dagger} T\right)_{\mathbb{Q}}$ à $\omega_{\mathfrak{X}^{\prime}} \otimes_{\mathcal{O}_{\mathfrak{X}^{\prime}}}$ $\mathcal{D}_{\mathfrak{X}^{\prime}}^{\dagger}\left({ }^{\dagger} T^{\prime}\right)_{\mathbb{Q}} \otimes \mathcal{O}_{\mathfrak{X}^{\prime}} \omega_{\mathfrak{X}^{\prime}}^{-1}$.

Notations 3.1.6. - Pour alléger les notations, on pose $\widetilde{\mathcal{O}}_{\mathfrak{X}}:=\mathcal{O}_{\mathfrak{X}}\left({ }^{\dagger} T\right)_{\mathbb{Q}}$ $\widetilde{\omega}_{\mathfrak{X}}:=\omega_{\mathfrak{X}} \otimes_{\mathcal{O}_{\mathfrak{X}}} \widetilde{\mathcal{O}}_{\mathfrak{X}}, \widetilde{\mathcal{D}}_{\mathfrak{X}}:=\mathcal{D}_{\mathfrak{X}}^{\dagger}\left({ }^{\dagger} T\right)_{\mathbb{Q}}$ (par défaut) ou $\mathcal{D}_{\mathfrak{X}}\left({ }^{\dagger} T\right)_{\mathbb{Q}}, \widetilde{\mathcal{D}}_{\mathfrak{X}^{\prime} \rightarrow \mathfrak{X}}:=$ $\lim _{\longrightarrow} \widehat{\mathcal{B}}_{\mathfrak{X}^{\prime}}^{(m)}\left(T^{\prime}\right) \widehat{\otimes}_{\mathcal{O}_{\mathfrak{X}^{\prime}}} \widehat{\mathcal{D}}_{\mathfrak{X}^{\prime} \rightarrow \mathfrak{X}, \mathbb{Q}}^{(m)}$ (par défaut) ou $\mathcal{O}_{\mathfrak{X}^{\prime}}\left({ }^{\dagger} T^{\prime}\right)_{\mathbb{Q}} \otimes_{\mathcal{O}_{\mathfrak{X}^{\prime}, \mathbb{Q}}} \mathcal{D}_{\mathfrak{X}^{\prime} \rightarrow \mathfrak{X}, \mathbb{Q}}$. De même en rajoutant des primes.

On notera $\left(\widetilde{\mathcal{D}}_{\mathfrak{X}} \otimes_{\widetilde{\mathcal{O}}_{\mathfrak{X}}} \widetilde{\omega}_{\mathfrak{X}}^{-1}\right)_{\mathrm{t}}$ le bimodule déduit de $\widetilde{\mathcal{D}}_{\mathfrak{X}} \otimes_{\widetilde{\mathcal{O}}_{\mathfrak{X}}} \widetilde{\omega}_{\mathfrak{X}}^{-1}$ via l'isomorphisme de transposition $\beta$ de $[5,1.3 .4 .3]$ (en d'autres termes, la structure 
gauche est la structure droite et vis versa). De même, $\left(\widetilde{\omega}_{\mathfrak{X}} \otimes_{\widetilde{\mathcal{O}}_{\mathfrak{X}}} \widetilde{\mathcal{D}}_{\mathfrak{X}}\right)_{\mathrm{t}}$ désignera le bimodule déduit de $\widetilde{\omega}_{\mathfrak{X}} \otimes_{\widetilde{\mathcal{O}}_{\mathfrak{X}}} \widetilde{\mathcal{D}}_{\mathfrak{X}}$ via l'isomorphisme de transposition $\delta$ de $[5$, 1.3.4.1]. De cette façon, les isomorphismes $\beta: \widetilde{\mathcal{D}}_{\mathfrak{X}} \otimes_{\widetilde{\mathcal{O}}_{\mathfrak{X}}} \widetilde{\omega}_{\mathfrak{X}}^{-1} \stackrel{\sim}{\longrightarrow}\left(\widetilde{\mathcal{D}}_{\mathfrak{X}} \otimes_{\widetilde{\mathcal{O}}_{\mathfrak{X}}} \widetilde{\omega}_{\mathfrak{X}}^{-1}\right)_{\mathrm{t}}$ et $\delta: \widetilde{\omega}_{\mathfrak{X}} \otimes_{\widetilde{\mathcal{O}}_{\mathfrak{X}}} \widetilde{\mathcal{D}}_{\mathfrak{X}} \stackrel{\sim}{\longrightarrow}\left(\widetilde{\omega}_{\mathfrak{X}} \otimes_{\widetilde{\mathcal{O}}_{\mathfrak{X}}} \widetilde{\mathcal{D}}_{\mathfrak{X}}\right)_{\mathrm{t}}$ sont bilinéaires.

3.1.7. - Soient $\mathcal{M} \in D_{\text {coh }}^{\mathrm{b}}\left(\mathcal{D}_{\mathfrak{X}}^{\dagger}\left({ }^{\dagger} T\right)_{\mathbb{Q}^{d}}\right), \mathcal{M}^{\prime} \in D_{\text {coh }}^{\mathrm{b}}\left(\mathcal{D}_{\mathfrak{X}^{\prime}}^{\dagger}\left({ }^{\dagger} T^{\prime}\right)_{\mathbb{Q}^{d}}\right), \mathcal{E} \in$ $D_{\text {coh }}^{\mathrm{b}}\left({ }^{\mathrm{g}} \mathcal{D}_{\mathfrak{X}}^{\dagger}\left({ }^{\dagger} T\right)_{\mathbb{Q}}\right), \mathcal{E}^{\prime} \in D_{\text {coh }}^{\mathrm{b}}\left({ }^{\mathrm{g}} \mathcal{D}_{\mathfrak{X}^{\prime}}^{\dagger}\left({ }^{\dagger} T^{\prime}\right)_{\mathbb{Q}}\right)$. Via les deux identifications de 3.1.6, on obtient :

$$
\begin{aligned}
f_{T}^{!}(\mathcal{E}) & =\mathcal{D}_{\mathfrak{X}^{\prime}}^{\dagger}\left({ }^{\dagger} T^{\prime}\right)_{\mathbb{Q}} \otimes_{f^{-1} \mathcal{D}_{\mathfrak{X}}^{\dagger}\left({ }^{\dagger} T\right)_{\mathbb{Q}}} f^{-1} \mathcal{E}, \\
f_{T}^{!}(\mathcal{M}) & =f^{-1} \mathcal{M} \otimes_{f^{-1} \mathcal{D}_{\mathfrak{X}}^{\dagger}\left({ }^{\dagger} T\right)_{\mathbb{Q}}}\left(\omega_{\mathfrak{X}^{\prime}} \otimes_{\mathcal{O}_{\mathfrak{X}^{\prime}}} \mathcal{D}_{\mathfrak{X}^{\prime}}^{\dagger}\left({ }^{\dagger} T^{\prime}\right)_{\mathbb{Q}} \otimes_{\mathcal{O}_{\mathfrak{X}^{\prime}}} \omega_{\mathfrak{X}^{\prime}}^{-1}\right), \\
f_{T,+}\left(\mathcal{E}^{\prime}\right) & =f_{*}\left(\left(\omega_{\mathfrak{X}^{\prime}} \otimes_{\mathcal{O}_{\mathfrak{X}^{\prime}}} \mathcal{D}_{\mathfrak{X}^{\prime}}^{\dagger}\left({ }^{\dagger} T^{\prime}\right)_{\mathbb{Q}} \otimes_{\mathcal{O}_{\mathfrak{X}^{\prime}}} \omega_{\mathfrak{X}^{\prime}}^{-1}\right) \otimes_{\mathcal{D}_{\mathfrak{X}^{\prime}}^{\dagger}\left({ }^{\dagger} T^{\prime}\right)_{\mathbb{Q}}} \mathcal{E}^{\prime}\right), \\
f_{T,+}\left(\mathcal{M}^{\prime}\right) & =f_{*}\left(\mathcal{M}^{\prime}\right) .
\end{aligned}
$$

On dispose des isomorphismes :

$$
\omega_{\mathfrak{X}^{\prime}} \otimes_{\mathcal{O}_{\mathfrak{X}^{\prime}}} f_{T}^{!}(\mathcal{E}) \stackrel{\sim}{\longrightarrow} f_{T}^{!}\left(\omega_{\mathfrak{X}} \otimes_{\mathcal{O}_{\mathfrak{X}}} \mathcal{E}\right), f_{T}^{!}(\mathcal{M}) \otimes_{\mathcal{O}_{\mathfrak{X}^{\prime}}} \omega_{\mathfrak{X}^{\prime}}^{-1} \stackrel{\sim}{\longrightarrow} f_{T}^{!}\left(\mathcal{M} \otimes_{\mathcal{O}_{\mathfrak{X}}} \omega_{\mathfrak{X}}^{-1}\right),
$$

$\omega_{\mathfrak{X}} \otimes_{\mathcal{O}_{\mathfrak{X}}} f_{T,+}\left(\mathcal{E}^{\prime}\right) \stackrel{\sim}{\longrightarrow} f_{T,+}\left(\omega_{\mathfrak{X}^{\prime}} \otimes_{\mathcal{O}_{\mathfrak{X}^{\prime}}} \mathcal{E}^{\prime}\right), f_{T,+}\left(\mathcal{M}^{\prime}\right) \otimes_{\mathcal{O}_{\mathfrak{X}}} \omega_{\mathfrak{X}}^{-1} \stackrel{\sim}{\longrightarrow} f_{T,+}\left(\mathcal{M}^{\prime} \otimes_{\mathcal{O}_{\mathfrak{X}^{\prime}}} \omega_{\mathfrak{X}^{\prime}}^{-1}\right)$

(pour le passage de droite à gauche, on pourra consulter [31, I.2]). Décrivons localement ces isomorphismes. Supposons donc $\mathfrak{X}$ affine muni de coordonnées locales $t_{1}, \ldots, t_{d}$. Notons $t_{1}^{\prime}, \ldots, t_{d}^{\prime}$ les coordonnées locales sur $\mathfrak{X}^{\prime}$ correspondantes et identifions $\omega_{\mathfrak{X}}$ à $\mathcal{O}_{\mathfrak{X}}$ grâce à la base $d t_{1} \wedge \cdots \wedge d t_{d}$, et $\omega_{\mathfrak{X}}^{-1}$ à $\mathcal{O}_{\mathfrak{X}}$ grâce à la base duale et de même avec des primes.

Avec ces identifications, on calcule d'abord que l'isomorphisme canonique (voir sa construction dans [31, I.2.1.2]) $\left(\widetilde{\mathcal{D}}_{\mathfrak{X}} \otimes_{\widetilde{\mathcal{O}}_{\mathfrak{X}}} \widetilde{\omega}_{\mathfrak{X}}^{-1}\right)_{\mathrm{t}} \otimes_{\widetilde{\mathcal{D}}_{\mathfrak{X}}}\left(\widetilde{\omega}_{\mathfrak{X}} \otimes_{\widetilde{\mathcal{O}}_{\mathfrak{X}}} \widetilde{\mathcal{D}}_{\mathfrak{X}}\right)_{\mathrm{t}} \stackrel{\sim}{\longrightarrow}$ $\widetilde{\mathcal{D}}_{\mathfrak{X}}$ envoie $P \otimes Q$ sur $Q P$, avec $P$ et $Q$ des sections locales de $\widetilde{\mathcal{D}}_{\mathfrak{X}}$. En particulier $1 \otimes 1$ s'envoie sur 1 . Il en découle que l'isomorphisme canonique de [31, I.2.2.(i)] $\mathcal{M} \otimes_{\widetilde{\mathcal{D}}_{\mathfrak{X}}} \mathcal{E} \stackrel{\sim}{\longrightarrow}\left(\widetilde{\omega}_{\mathfrak{X}} \otimes_{\widetilde{\mathcal{O}}_{\mathfrak{X}}} \mathcal{E}\right) \otimes_{\widetilde{\mathcal{D}}_{\mathfrak{X}}}\left(\mathcal{M} \otimes_{\widetilde{\mathcal{O}}_{\mathfrak{X}}} \widetilde{\omega}_{\mathfrak{X}}^{-1}\right)$ expédie $x \otimes e$ sur $e \otimes x$, où $x$ et $e$ sont des sections locales respectives de $\mathcal{M}$ et $\mathcal{E}$. Or, l'isomorphisme $\omega_{\mathfrak{X}^{\prime}} \otimes_{\mathcal{O}_{\mathfrak{X}^{\prime}}} f_{T}^{!}(\mathcal{E}) \stackrel{\sim}{\longrightarrow} f_{T}^{!}\left(\omega_{\mathfrak{X}} \otimes_{\mathcal{O}_{\mathfrak{X}}} \mathcal{E}\right)$ est le composé suivant :

$\omega_{\mathfrak{X}^{\prime}} \otimes_{\mathcal{O}_{\mathfrak{X}^{\prime}}}\left(\widetilde{\mathcal{D}}_{\mathfrak{X}^{\prime}} \otimes_{f^{-1}} \widetilde{\mathcal{D}}_{\mathfrak{X}} f^{-1} \mathcal{E}\right) \stackrel{\sim}{\longrightarrow} \omega_{\mathfrak{X}^{\prime}} \otimes_{\mathcal{O}_{\mathfrak{X}^{\prime}}}\left(f^{-1}\left(\widetilde{\omega}_{\mathfrak{X}} \otimes_{\widetilde{\mathcal{O}}_{\mathfrak{X}}} \mathcal{E}\right) \otimes_{f^{-1}}^{\mathrm{d}} \widetilde{\mathcal{D}}_{\mathfrak{X}}\left(\widetilde{\mathcal{D}}_{\mathfrak{X}^{\prime}} \otimes_{\widetilde{\mathcal{O}}_{\mathfrak{X}^{\prime}}} \widetilde{\omega}_{\mathfrak{X}^{\prime}}^{-1}\right)\right) \stackrel{\sim}{\longrightarrow}$

$$
\stackrel{\sim}{\longrightarrow} f^{-1}\left(\widetilde{\omega}_{\mathfrak{X}} \otimes_{\widetilde{\mathcal{O}}_{\mathfrak{X}}} \mathcal{E}\right) \otimes_{f^{-1}} \widetilde{\mathcal{D}}_{\mathfrak{X}}\left(\omega_{\mathfrak{X}^{\prime}} \otimes_{\mathcal{O}_{\mathfrak{X}^{\prime}}} \widetilde{\mathcal{D}}_{\mathfrak{X}^{\prime}} \otimes_{\widetilde{\mathcal{O}}_{\mathfrak{X}^{\prime}}} \widetilde{\omega}_{\mathfrak{X}^{\prime}}^{-1}\right),
$$

où le symbole « $\mathrm{d}$ » signifie que l'on a pris, pour calculer le produit tensoriel, la structure droite (i.e. la structure tordue) de $\widetilde{\mathcal{D}}_{\mathfrak{X}^{\prime}} \otimes_{\widetilde{\mathcal{O}}_{\mathfrak{X}^{\prime}}} \widetilde{\omega}_{\mathfrak{X}^{\prime}}^{-1}$, et où le premier isomorphisme découle de [31, I.2.2.(i)]. Le composé 3.1.7.3 envoie $P^{\prime} \otimes x$ sur 
$x \otimes P^{\prime}$, où $P^{\prime}$ et $x$ sont des sections locales respectives de $\widetilde{\mathcal{D}}_{\mathfrak{X}^{\prime}}$ et $\mathcal{E}$. On bénéficie de descriptions analogues pour les trois autres isomorphismes de 3.1.7.1 et 3.1.7.2.

De plus, on définit les opérations analogues en remplaçant « $\mathcal{D}^{\dagger} »$ par $« \mathcal{D} »$. Si un risque de confusion est à craindre, on notera $f_{T}^{!^{\dagger}}$ (resp. $f_{T,+}^{\dagger}$ ) pour $f_{T}^{!}$ (resp. $\left.f_{T,+}\right)$. Pour tout $\mathcal{E} \in D_{\text {coh }}^{\mathrm{b}}\left({ }^{\mathrm{g}} \mathcal{D}_{\mathfrak{X}}\left({ }^{\dagger} T\right)_{\mathbb{Q}}\right)$, on dispose d'un isomorphisme canonique

$$
f_{T}^{!^{\dagger}}\left(\mathcal{D}_{\mathfrak{X}}^{\dagger}\left({ }^{\dagger} T\right)_{\mathbb{Q}} \otimes_{\mathcal{D}_{\mathfrak{X}}\left({ }^{\dagger} T\right)_{\mathbb{Q}}} \mathcal{E}\right) \stackrel{\sim}{\longrightarrow} \mathcal{D}_{\mathfrak{X}^{\prime}}^{\dagger}\left({ }^{\dagger} T^{\prime}\right)_{\mathbb{Q}} \otimes_{\mathcal{D}_{\mathfrak{X}^{\prime}}\left({ }^{\dagger} T^{\prime}\right)_{\mathbb{Q}}} f_{T}^{!}(\mathcal{E}) .
$$

3.1.8. - On dispose des isomorphismes canoniques $f_{T, \mathrm{~g}}^{!}\left(\widetilde{\mathcal{D}}_{\mathfrak{X}} \otimes_{\widetilde{\mathcal{O}}_{\mathfrak{X}}} \widetilde{\omega}_{\mathfrak{X}}^{-1}\right) \stackrel{\sim}{\longrightarrow}$ $\widetilde{\mathcal{D}}_{\mathfrak{X}^{\prime}} \otimes_{\widetilde{\mathcal{O}}_{\mathfrak{X}^{\prime}}} \widetilde{\omega}_{\mathfrak{X}^{\prime}}^{-1}$ et $f_{T, \mathrm{~d}}^{!}\left(\left(\widetilde{\mathcal{D}}_{\mathfrak{X}} \otimes_{\widetilde{\mathcal{O}}_{\mathfrak{X}}} \widetilde{\omega}_{\mathfrak{X}}^{-1}\right)_{\mathrm{t}}\right) \stackrel{\sim}{\longrightarrow}\left(\widetilde{\mathcal{D}}_{\mathfrak{X}^{\prime}} \otimes_{\widetilde{\mathcal{O}}_{\mathfrak{X}^{\prime}}} \widetilde{\omega}_{\mathfrak{X}^{\prime}}^{-1}\right)_{\mathrm{t}}$. De plus, on bénéficie du composé suivant :

$$
\begin{aligned}
& f_{T, \mathrm{~d}}^{!}\left(\widetilde{\mathcal{D}}_{\mathfrak{X}} \otimes_{\widetilde{\mathcal{O}}_{\mathfrak{X}}} \widetilde{\omega}_{\mathfrak{X}}^{-1}\right)=\widetilde{\mathcal{D}}_{\mathfrak{X}^{\prime}} \otimes_{f^{-1}}^{\mathrm{dd}} \widetilde{\mathcal{D}}_{\mathfrak{X}} f^{-1}\left(\widetilde{\mathcal{D}}_{\mathfrak{X}} \otimes_{\widetilde{\mathcal{O}}_{\mathfrak{X}}} \widetilde{\omega}_{\mathfrak{X}}^{-1}\right) \stackrel{\sim}{\longrightarrow} \\
& \stackrel{\sim}{\longrightarrow} f^{-1} \widetilde{\mathcal{D}}_{\mathfrak{X}} \otimes_{f^{-1} \widetilde{\mathcal{D}}_{\mathfrak{X}}}^{\mathrm{dd}}\left(\widetilde{\mathcal{D}}_{\mathfrak{X}^{\prime}} \otimes_{\widetilde{\mathcal{O}}_{\mathfrak{X}^{\prime}}} \widetilde{\omega}_{\mathfrak{X}^{\prime}}^{-1}\right) \stackrel{\sim}{\longrightarrow}\left(\widetilde{\mathcal{D}}_{\mathfrak{X}^{\prime}} \otimes_{\widetilde{\mathcal{O}}_{\mathfrak{X}^{\prime}}} \widetilde{\omega}_{\mathfrak{X}^{\prime}}^{-1}\right)_{\mathrm{t}},
\end{aligned}
$$

où les symboles « $\mathrm{d}$ » signifient que l'on choisit dans le calcul des produits tensoriels les structures droites des bimodules respectifs, et où le premier isomorphisme découle de [31, I.2.2.(i)]. De manière analogue à 3.1.8.1, on construit $f_{T, \mathrm{~g}}^{!}\left(\left(\widetilde{\mathcal{D}}_{\mathfrak{X}} \otimes_{\widetilde{\mathcal{O}}_{\mathfrak{X}}} \widetilde{\omega}_{\mathfrak{X}}^{-1}\right)_{\mathrm{t}}\right) \stackrel{\sim}{\longrightarrow} \widetilde{\mathcal{D}}_{\mathfrak{X}^{\prime}} \otimes_{\widetilde{\mathcal{O}}_{\mathfrak{X}^{\prime}}} \widetilde{\omega}_{\mathfrak{X}^{\prime}}^{-1}$.

Par un calcul local, on vérifie que les diagrammes

$$
\begin{aligned}
& f_{T, \mathrm{~g}}^{!}\left(\widetilde{\mathcal{D}}_{\mathfrak{X}} \otimes_{\widetilde{\mathcal{O}}_{\mathfrak{X}}} \widetilde{\omega}_{\mathfrak{X}}^{-1}\right) \stackrel{\beta}{\sim} f_{T, \mathrm{~g}}^{!}\left(\left(\widetilde{\mathcal{D}}_{\mathfrak{X}} \otimes_{\widetilde{\mathcal{O}}_{\mathfrak{X}}} \widetilde{\omega}_{\mathfrak{X}}^{-1}\right)_{\mathrm{t}}\right) \quad f_{T, \mathrm{~d}}^{!}\left(\left(\widetilde{\mathcal{D}}_{\mathfrak{X}} \otimes_{\widetilde{\mathcal{O}}_{\mathfrak{X}}} \widetilde{\omega}_{\mathfrak{X}}^{-1}\right)_{\mathrm{t}}\right) \stackrel{\beta}{\sim} f_{T, \mathrm{~d}}^{!}\left(\widetilde{\mathcal{D}}_{\mathfrak{X}} \otimes_{\widetilde{\mathcal{O}}_{\mathfrak{X}}} \widetilde{\omega}_{\mathfrak{X}}^{-1}\right) \\
& \sim \downarrow \quad \sim \downarrow \quad \sim \downarrow \quad \sim \downarrow \\
& \widetilde{\mathcal{D}}_{\mathfrak{X}^{\prime}} \otimes_{\widetilde{\mathcal{O}}_{\mathfrak{X}^{\prime}}} \widetilde{\omega}_{\mathfrak{X}^{\prime}}^{-1}=\left(\widetilde{\mathcal{D}}_{\mathfrak{X}^{\prime}} \otimes_{\widetilde{\mathcal{O}}_{\mathfrak{X}^{\prime}}} \widetilde{\omega}_{\mathfrak{X}^{\prime}}^{-1}, \quad\left(\widetilde{\mathcal{D}}_{\mathfrak{X}^{\prime}} \otimes_{\widetilde{\mathcal{O}}_{\mathfrak{X}^{\prime}}} \widetilde{\omega}_{\mathfrak{X}^{\prime}}^{-1}\right)_{\mathrm{t}}=\left(\widetilde{\mathcal{D}}_{\mathfrak{X}^{\prime}} \otimes_{\widetilde{\mathcal{O}}_{\mathfrak{X}^{\prime}}} \widetilde{\omega}_{\mathfrak{X}^{\prime}}^{-1}\right)_{\mathrm{t}},\right.
\end{aligned}
$$

sont commutatifs. Lorsque $f$ est l'identité, les flèches de gauche des deux carrés sont égaux à l'identité et celles de droite s'identifient à $\beta$.

\subsection{Sur la commutation des foncteurs duaux aux images inverses par un isomor- phisme}

Notations 3.2.1. - Commençons par quelques notations et rappels sur les foncteurs duaux.

Pour tout $\mathcal{E} \in D_{\text {parf }}\left({ }^{\mathrm{g}} \mathcal{D}_{\mathfrak{X}}\left({ }^{\dagger} T\right)_{\mathbb{Q}}\right)$, le complexe dual $\mathcal{D}_{\mathfrak{X}}\left({ }^{\dagger} T\right)_{\mathbb{Q}}$-linéaire de $\mathcal{E}$ au sens de Virrion (voir [31, I.3.2]) est défini en posant :

$$
\mathbb{D}_{\mathfrak{X}, T}(\mathcal{E}):=\mathbb{R} \mathcal{H}^{o m} m_{\mathcal{D}_{\mathfrak{X}}\left({ }^{\dagger} T\right)_{\mathbb{Q}}}\left(\mathcal{E}, \mathcal{D}_{\mathfrak{X}}\left({ }^{\dagger} T\right)_{\mathbb{Q}} \otimes_{\mathcal{O}_{\mathfrak{X}}} \omega_{\mathfrak{X}}^{-1}\right)\left[d_{X}\right] .
$$


De même, pour tous $\mathcal{E} \in D_{\text {parf }}\left({ }^{\mathrm{g}} \mathcal{D}_{\mathfrak{X}, \mathbb{Q}}^{\dagger}\left({ }^{\dagger} T\right)\right), \mathcal{M} \in D_{\text {parf }}\left(\mathcal{D}_{\mathfrak{X}, \mathbb{Q}}^{\dagger}\left({ }^{\dagger} T\right)^{\mathrm{d}}\right)$, leurs complexes duaux $\mathcal{D}_{\mathfrak{X}, \mathbb{Q}}^{\dagger}\left({ }^{\dagger} T\right)$-linéaires sont définis comme suit :

$$
\begin{gathered}
\mathbb{D}_{\mathfrak{X}, T}^{\dagger}(\mathcal{E}):=\mathbb{R} \mathcal{H} o m_{\widetilde{\mathcal{D}}_{\mathfrak{X}}}\left(\mathcal{E}, \widetilde{\mathcal{D}}_{\mathfrak{X}} \otimes_{\widetilde{\mathcal{O}}_{\mathfrak{X}}} \widetilde{\omega}_{\mathfrak{X}}^{-1}\right)\left[d_{X}\right], \\
\mathbb{D}_{\mathfrak{X}, T}^{\dagger}(\mathcal{E}):=\mathbb{R H}_{\widetilde{\mathcal{D}}_{\mathfrak{X}}}\left(\mathcal{E},\left(\widetilde{\mathcal{D}}_{\mathfrak{X}} \otimes_{\widetilde{\mathcal{O}}_{\mathfrak{X}}} \widetilde{\omega}_{\mathfrak{X}}^{-1}\right)_{\mathrm{t}}\right)\left[d_{X}\right], \\
\mathbb{D}_{\mathfrak{X}, T}^{\dagger}(\mathcal{M}):=\widetilde{\omega}_{\mathfrak{X}} \otimes_{\widetilde{\mathcal{O}}_{\mathfrak{X}}} \mathbb{R} \mathcal{H} m_{\widetilde{\mathcal{D}}_{\mathfrak{X}}}\left(\mathcal{M}, \widetilde{\mathcal{D}}_{\mathfrak{X}}\right)\left[d_{X}\right] \stackrel{\sim}{\longrightarrow} \mathbb{R} \mathcal{H}_{\widetilde{\mathcal{D}}_{\mathfrak{X}}}\left(\mathcal{M},\left(\widetilde{\omega}_{\mathfrak{X}} \otimes_{\widetilde{\mathcal{O}}_{\mathfrak{X}}} \widetilde{\mathcal{D}}_{\mathfrak{X}}\right)_{\mathrm{t}}\right)\left[d_{X}\right], \\
\mathbb{D}_{\mathfrak{X}, T}^{\prime \dagger}(\mathcal{M}):=\mathbb{R} \operatorname{Hom}_{\widetilde{\mathcal{D}}_{\mathfrak{X}}}\left(\mathcal{M}, \widetilde{\omega}_{\mathfrak{X}} \otimes_{\widetilde{\mathcal{O}}_{\mathfrak{X}}} \widetilde{\mathcal{D}}_{\mathfrak{X}}\right)\left[d_{X}\right] .
\end{gathered}
$$

Le foncteur dual commutant à l'extension des scalaires ([31]), si $\mathcal{E} \in$ $D_{\text {parf }}\left({ }^{g} \mathcal{D}_{\mathfrak{X}}\left({ }^{\dagger} T\right)_{\mathbb{Q}}\right)$,

$$
\mathbb{D}_{\mathfrak{X}, T}^{\dagger}\left(\mathcal{D}_{\mathfrak{X}}^{\dagger}\left({ }^{\dagger} T\right)_{\mathbb{Q}} \otimes_{\mathcal{D}_{\mathfrak{X}}\left({ }^{\dagger} T\right)_{\mathbb{Q}}} \mathcal{E}\right) \stackrel{\sim}{\longrightarrow} \mathcal{D}_{\mathfrak{X}}^{\dagger}\left({ }^{\dagger} T\right)_{\mathbb{Q}} \otimes_{\mathcal{D}_{\mathfrak{X}}\left({ }^{\dagger} T\right)_{\mathbb{Q}}} \mathbb{D}_{\mathfrak{X}, T}(\mathcal{E}) .
$$

Si aucune confusion avec 3.2.1.1 n'est à craindre, on omettra d'indiquer le symbole «†».

On dispose des isomorphismes canoniques :

$\mathbb{D}_{\mathfrak{X}, T}^{\prime}(\mathcal{M}) \otimes_{\widetilde{\mathcal{O}}_{\mathfrak{X}}} \widetilde{\omega}_{\mathfrak{X}}^{-1} \stackrel{\sim}{\longrightarrow} \mathbb{R} \mathcal{H} o m_{\mathrm{g}} \widetilde{\mathcal{D}}_{\mathfrak{X}}\left(\mathcal{M} \otimes_{\widetilde{\mathcal{O}}_{\mathfrak{X}}} \widetilde{\omega}_{\mathfrak{X}}^{-1}, \widetilde{\mathcal{D}}_{\mathfrak{X}}\right) \otimes_{\widetilde{\mathcal{O}}_{\mathfrak{X}}} \widetilde{\omega}_{\mathfrak{X}}^{-1}\left[d_{X}\right] \stackrel{\sim}{\longrightarrow} \mathbb{D}_{\mathfrak{X}, T}\left(\mathcal{M} \otimes_{\widetilde{\mathcal{O}}_{\mathfrak{X}}} \widetilde{\omega}_{\mathfrak{X}}^{-1}\right)$,

$\widetilde{\omega}_{\mathfrak{X}} \otimes_{\widetilde{\mathcal{O}}_{\mathfrak{X}}} \mathbb{D}_{\mathfrak{X}, T}^{\prime}(\mathcal{E}) \stackrel{\sim}{\longrightarrow} \widetilde{\omega}_{\mathfrak{X}} \otimes_{\widetilde{\mathcal{O}}_{\mathfrak{X}}} \mathbb{R} \mathcal{H} o m_{\widetilde{\mathcal{D}}_{\mathfrak{X}}{ }^{d}}\left(\widetilde{\omega}_{\mathfrak{X}} \otimes_{\widetilde{\mathcal{O}}_{\mathfrak{X}}} \mathcal{E}, \widetilde{\mathcal{D}}_{\mathfrak{X}}\right)\left[d_{X}\right] \stackrel{\sim}{\longrightarrow} \mathbb{D}_{\mathfrak{X}, T}\left(\widetilde{\omega}_{\mathfrak{X}} \otimes_{\widetilde{\mathcal{O}}_{\mathfrak{X}}} \mathcal{E}\right)$.

Les isomorphismes 3.2.1.3 et 3.2.1.4 n'utilisent pas celui de transposition, ce qui facilite les calculs locaux. En effet, supposons $\mathfrak{X}$ affine et muni de coordonnées locales $t_{1}, \ldots, t_{d}$. Notons $t_{1}^{\prime}, \ldots, t_{d}^{\prime}$ les coordonnées locales sur $\mathfrak{X}^{\prime}$ correspondantes et identifions $\omega_{\mathfrak{X}}$ à $\mathcal{O}_{\mathfrak{X}}$ grâce à la base $d t_{1} \wedge \cdots \wedge d t_{d}$, et $\omega_{\mathfrak{X}}^{-1}$ à $\mathcal{O}_{\mathfrak{X}}$ grâce à la base duale et de même avec des primes. Les isomorphismes 3.2.1.3 et 3.2.1.4 s'identifient alors à l'identité.

Enfin, on remarque que dans le lemme 3.2.3 l'isomorphisme de transposition n'apparaît pas non plus.

Notations 3.2.2. - • Pour tout $\mathcal{M}^{\prime} \in D_{\text {parf }}\left(\mathcal{D}_{\mathfrak{X}^{\prime}, \mathbb{Q}}^{\dagger}\left({ }^{\dagger} T^{\prime}\right)^{\mathrm{d}}\right)$, l'isomorphisme de dualité relative $([12,1.2 .7]) f_{T,+} \circ \mathbb{D}_{\mathfrak{X}^{\prime}, T^{\prime}}\left(\mathcal{M}^{\prime}\right) \stackrel{\sim}{\longrightarrow} \mathbb{D}_{\mathfrak{X}, T} \circ f_{T,+}\left(\mathcal{M}^{\prime}\right)$ sera noté $\chi$. En fait, via les isomorphismes de transposition $\delta$ de $[5,1.3 .4 .1]$, nous avions d'abord construit l'isomorphisme $f_{T,+} \circ \mathbb{D}_{\mathfrak{X}^{\prime}, T^{\prime}}^{\prime}\left(\mathcal{M}^{\prime}\right) \stackrel{\sim}{\longrightarrow} \mathbb{D}_{\mathfrak{X}, T}^{\prime} \circ f_{T,+}\left(\mathcal{M}^{\prime}\right)$, que l'on désignera encore par $\chi$.

- Pour tout $\mathcal{E} \in D_{\text {parf }}\left({ }^{\mathrm{g}} \mathcal{D}_{\mathfrak{X}, \mathbb{Q}}^{\dagger}\left({ }^{\dagger} T\right)\right)$, on notera :

$$
\operatorname{proj}^{\prime}: f_{+}\left(\widetilde{\omega}_{\mathfrak{X}^{\prime}}\right) \otimes_{\widetilde{\mathcal{O}}_{\mathfrak{X}}} \mathcal{E}=f_{*}\left(\widetilde{\omega}_{\mathfrak{X}^{\prime}}\right) \otimes_{\widetilde{\mathcal{O}}_{\mathfrak{X}}} \mathcal{E} \stackrel{\sim}{\longrightarrow} f_{*}\left(\widetilde{\omega}_{\mathfrak{X}^{\prime}}\right) \otimes_{\widetilde{\mathcal{O}}_{\mathfrak{X}}} f_{*}\left(\widetilde{\mathcal{D}}_{\mathfrak{X}^{\prime}} \otimes_{f^{-1} \widetilde{\mathcal{D}}_{\mathfrak{X}}} f^{-1} \mathcal{E}\right)
$$

$$
\stackrel{\sim}{\longrightarrow} f_{*}\left(\widetilde{\omega}_{\mathfrak{X}^{\prime}} \otimes_{\widetilde{\mathcal{O}}_{\mathfrak{X}^{\prime}}}\left(\widetilde{\mathcal{D}}_{\mathfrak{X}^{\prime}} \otimes_{f^{-1} \widetilde{\mathcal{D}}_{\mathfrak{X}}} f^{-1} \mathcal{E}\right)\right)=f_{+}\left(\widetilde{\omega}_{\mathfrak{X}^{\prime}} \otimes_{\widetilde{\mathcal{O}}_{\mathfrak{X}^{\prime}}} f_{T}^{!}(\mathcal{E})\right) .
$$


On obtient en particulier proj' $: f_{*}\left(\widetilde{\omega}_{\mathfrak{X}^{\prime}}\right) \otimes_{\widetilde{\mathcal{O}}_{\mathfrak{X}}} \widetilde{\mathcal{D}}_{\mathfrak{X}} \stackrel{\sim}{\longrightarrow} f_{*}\left(\widetilde{\omega}_{\mathfrak{X}^{\prime}} \otimes_{\widetilde{\mathcal{O}}_{\mathfrak{X}^{\prime}}} \widetilde{\mathcal{D}}_{\mathfrak{X}^{\prime}}\right)$, ce dernier étant par fonctorialité $\widetilde{\mathcal{D}}_{\mathfrak{X}}$-bilinéaire. On vérifie par un calcul que cet isomorphisme proj' correspond bien à l'isomorphisme canonique lorsque $f$ est un morphisme quasi-compact et quasi-séparé quelconque (pour la construction dans le cas général, voir [9, 1.2.27] ou [16, 1.4.1]).

- Le morphisme trace $: f_{*}\left(\widetilde{\omega}_{\mathfrak{X}^{\prime}}\right)=f_{+}\left(\widetilde{\omega}_{\mathfrak{X}^{\prime}}\right) \rightarrow \widetilde{\omega}_{\mathfrak{X}}$ sera noté Tr.

Lemme 3.2.3. - Pour tout $\mathcal{M}^{\prime} \in D_{\text {parf }}\left(\mathcal{D}_{\mathfrak{X}^{\prime}, \mathbb{Q}}^{\dagger}\left({ }^{\dagger} T^{\prime}\right)^{\mathrm{d}}\right)$, avec les notations 3.2.1 et 3.2.2, le diagramme ci-après

$$
\begin{aligned}
& f_{*} \mathbb{R H o m}_{\widetilde{\mathcal{D}}_{\mathfrak{X}^{\prime}}}\left(\mathcal{M}^{\prime}, \widetilde{\omega}_{\mathfrak{X}^{\prime}} \otimes_{\widetilde{\mathcal{O}}_{\mathfrak{X}^{\prime}}} \widetilde{\mathcal{D}}_{\mathfrak{X}^{\prime}}\right)\left[d_{X^{\prime}}\right]=f_{T,+} \circ \mathbb{D}_{\mathfrak{X}^{\prime}, T^{\prime}}^{\prime}\left(\mathcal{M}^{\prime}\right) \\
& \sim \downarrow \\
& f_{*} \mathbb{R} \mathcal{H o m}_{f^{-1} \widetilde{\mathcal{D}}_{\mathfrak{X}}}\left(\mathcal{M}^{\prime}, \widetilde{\omega}_{\mathfrak{X}^{\prime}} \otimes_{\widetilde{\mathcal{O}}_{\mathfrak{X}^{\prime}}} \widetilde{\mathcal{D}}_{\mathfrak{X}^{\prime}}\right)\left[d_{X^{\prime}}\right] \\
& \sim \downarrow f_{*} \\
& \mathbb{R H} \operatorname{Hom}_{\widetilde{\mathcal{D}}_{\mathfrak{X}}}\left(f_{*} \mathcal{M}^{\prime}, f_{*}\left(\widetilde{\omega}_{\mathfrak{X}^{\prime}} \otimes_{\widetilde{\mathcal{O}}_{\mathfrak{X}^{\prime}}} \widetilde{\mathcal{D}}_{\mathfrak{X}^{\prime}}\right)\right)\left[d_{X^{\prime}}\right] \\
& \operatorname{proj}^{\prime} \uparrow \sim \\
& \left.\mathbb{R H o m}_{\widetilde{\mathcal{D}}_{\mathfrak{X}}}\left(f_{*} \mathcal{M}^{\prime}, f_{*}\left(\widetilde{\omega}_{\mathfrak{X}^{\prime}}\right) \otimes_{\widetilde{\mathcal{O}}_{\mathfrak{X}}} \widetilde{\mathcal{D}}_{\mathfrak{X}}\right)\right)\left[d_{X^{\prime}}\right] \\
& \sim \downarrow \operatorname{Tr} \\
& \mathbb{R} \mathcal{H}_{\widetilde{\mathcal{D}}_{\mathfrak{X}}}\left(f_{*} \mathcal{M}^{\prime}, \widetilde{\omega}_{\mathfrak{X}} \otimes_{\widetilde{\mathcal{O}}_{\mathfrak{X}}} \widetilde{\mathcal{D}}_{\mathfrak{X}}\right)\left[d_{X}\right]=\mathbb{D}_{\mathfrak{X}, T}^{\prime} \circ f_{T,+}\left(\mathcal{M}^{\prime}\right),
\end{aligned}
$$

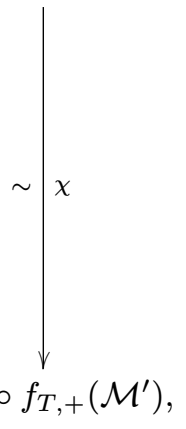

où l'isomorphisme à droite en haut dérive de l'identification entre $\widetilde{\mathcal{D}}_{\mathfrak{X}^{\prime}}$ et $\widetilde{\mathcal{D}}_{\mathfrak{X}^{\prime} \rightarrow \mathfrak{X}}$, est commutatif.

Démonstration. - Modulo l'identification entre $\widetilde{\mathcal{D}}_{\mathfrak{X}^{\prime}}$ et $\widetilde{\mathcal{D}}_{\mathfrak{X}^{\prime} \rightarrow \mathfrak{X}}$, le morphisme $[12,1.2 .7 .8]$ est égal au morphisme Troproj ${ }^{\prime-1}: f_{*}\left(\widetilde{\omega}_{\mathfrak{X}^{\prime}} \otimes_{\widetilde{\mathcal{O}}_{\mathfrak{X}^{\prime}}} \widetilde{\mathcal{D}}_{\mathfrak{X}^{\prime}}\right) \stackrel{\sim}{\longrightarrow} \widetilde{\omega}_{\mathfrak{X}} \otimes_{\widetilde{\mathcal{O}}_{\mathfrak{X}}}$ $\widetilde{\mathcal{D}}_{\mathfrak{X}}$. De plus, le composé de $[12,1.2 .7 .2]$ correspond à $f_{*} \mathbb{R} \mathcal{H}$ om $\widetilde{\mathcal{D}}_{\mathfrak{X}^{\prime}}\left(\mathcal{M}^{\prime}, \widetilde{\omega}_{\mathfrak{X}^{\prime}} \otimes_{\widetilde{\mathcal{O}}_{\mathfrak{X}^{\prime}}}\right.$ $\left.\widetilde{\mathcal{D}}_{\mathfrak{X}^{\prime}}\right)\left[d_{X^{\prime}}\right] \stackrel{\sim}{\longrightarrow} \mathbb{R} \mathcal{H o m}_{\widetilde{\mathcal{D}}_{\mathfrak{X}}}\left(f_{*} \mathcal{M}^{\prime}, f_{*}\left(\widetilde{\omega}_{\mathfrak{X}^{\prime}} \otimes_{\widetilde{\mathcal{O}}_{\mathfrak{X}^{\prime}}} \widetilde{\mathcal{D}}_{\mathfrak{X}^{\prime}}\right)\right)\left[d_{X^{\prime}}\right]$ de 3.2.3.1. D'où le résultat par construction de l'isomorphisme $\chi$ de $[12,1.2 .7]$.

3.2.4. - Dans ce paragraphe 3.2.4, supposons $f$ seulement propre. Pour la commodité du lecteur, rappelons que l'on dispose, pour tous $\mathcal{M}^{\prime} \in$ $D_{\text {parf }}\left(\mathcal{D}_{\mathfrak{X}^{\prime}, \mathbb{Q}}^{\dagger}\left({ }^{\dagger} T^{\prime}\right)^{\mathrm{d}}\right), \mathcal{E} \in D_{\text {parf }}\left({ }^{\mathrm{g}} \mathcal{D}_{\mathfrak{X}, \mathbb{Q}}^{\dagger}\left({ }^{\dagger} T\right)\right)$, de l'isomorphisme composé :

$$
\begin{aligned}
& \mathbb{R} f_{*} \mathbb{R} \mathcal{H o m}_{\widetilde{\mathcal{D}}_{\mathfrak{X}^{\prime}}}\left(\mathcal{M}^{\prime}, f_{T}^{!}\left(\widetilde{\omega}_{\mathfrak{X}} \otimes_{\widetilde{\mathcal{O}}_{\mathfrak{X}}} \mathcal{E}\right)\right) \stackrel{\sim}{\longrightarrow} \mathbb{R} f_{*} \mathbb{R} \mathcal{H} o m_{\widetilde{\mathcal{D}}_{\mathfrak{X}^{\prime}}}\left(\mathcal{M}^{\prime}, \widetilde{\omega}_{\mathfrak{X}^{\prime}} \otimes_{\widetilde{\mathcal{O}}_{\mathfrak{X}^{\prime}}} f_{T}^{!}(\mathcal{E})\right) \\
& =\mathbb{R} f_{*} \mathbb{R} \mathcal{H o m}_{\widetilde{\mathcal{D}}_{\mathfrak{X}^{\prime}}}\left(\mathcal{M}^{\prime}, \widetilde{\omega}_{\mathfrak{X}^{\prime}} \otimes_{\widetilde{\mathcal{O}}_{\mathfrak{X}^{\prime}}} \widetilde{\mathcal{D}}_{\mathfrak{X}^{\prime} \rightarrow \mathfrak{X}} \otimes_{f^{-1} \widetilde{\mathcal{D}}_{\mathfrak{X}}}^{\mathbb{L}} f^{-1} \mathcal{E}\right)\left[d_{X^{\prime} / X}\right] \\
& \underset{\operatorname{proj}_{f}}{\sim}\left(\mathbb{R} f_{*} \mathbb{R} \mathcal{H} \text { om }_{\widetilde{\mathcal{D}}_{\mathfrak{X}^{\prime}}}\left(\mathcal{M}^{\prime}, \widetilde{\omega}_{\mathfrak{X}^{\prime}} \otimes_{\widetilde{\mathcal{O}}_{\mathfrak{X}^{\prime}}} \widetilde{\mathcal{D}}_{\mathfrak{X}^{\prime} \rightarrow \mathfrak{X}}\right)\right) \otimes \otimes_{\widetilde{\mathcal{D}}_{\mathfrak{X}}}^{\mathbb{L}} \mathcal{E}\left[d_{X^{\prime} / X}\right] \\
& \stackrel{\sim}{\otimes}\left(\mathbb{R} f_{*}\left(\mathbb{R} \mathcal{H} m_{\widetilde{\mathcal{D}}_{\mathfrak{X}^{\prime}}}\left(\mathcal{M}^{\prime}, \widetilde{\omega}_{\mathfrak{X}^{\prime}} \otimes_{\widetilde{\mathcal{O}}_{\mathfrak{X}^{\prime}}} \widetilde{\mathcal{D}}_{\mathfrak{X}^{\prime}}\right) \otimes_{\widetilde{\mathcal{D}}_{\mathfrak{X}^{\prime}}}^{\mathbb{L}} \widetilde{\mathcal{D}}_{\mathfrak{X}^{\prime} \rightarrow \mathfrak{X}}\right)\right) \otimes_{\widetilde{\mathcal{D}}_{\mathfrak{X}}}^{\mathbb{L}} \mathcal{E}\left[d_{X^{\prime} / X}\right]
\end{aligned}
$$




$$
\begin{aligned}
& \underset{\chi}{\stackrel{\sim}{\longrightarrow}} \mathbb{R} \mathcal{H o m}_{\widetilde{\mathcal{D}}_{\mathfrak{X}}}\left(f_{T,+}\left(\mathcal{M}^{\prime}\right), \widetilde{\omega}_{\mathfrak{X}} \otimes_{\widetilde{\mathcal{O}}_{\mathfrak{X}}} \widetilde{\mathcal{D}}_{\mathfrak{X}^{\prime}}\right) \otimes_{\widetilde{\mathcal{D}}_{\mathfrak{X}}}^{\mathbb{L}} \mathcal{E} \\
& \underset{\otimes}{\stackrel{\sim}{\longrightarrow}} \mathbb{R} \mathcal{H o m}_{\widetilde{\mathcal{D}}_{\mathfrak{X}}}\left(f_{T,+}\left(\mathcal{M}^{\prime}\right), \widetilde{\omega}_{\mathfrak{X}} \otimes_{\widetilde{\mathcal{O}}_{\mathfrak{X}}} \mathcal{E}\right) \text {. }
\end{aligned}
$$

En lui appliquant $H^{0} \circ \Gamma(\mathfrak{X},-)$, on obtient l'isomorphisme d'adjonction suivant :

$$
\operatorname{adj}: \operatorname{Hom}_{\widetilde{\mathcal{D}}_{\mathfrak{X}^{\prime}}}\left(\mathcal{M}^{\prime}, f_{T}^{!}\left(\widetilde{\omega}_{\mathfrak{X}} \otimes_{\widetilde{\mathcal{O}}_{\mathfrak{X}}} \mathcal{E}\right)\right) \stackrel{\sim}{\longrightarrow} \operatorname{Hom}_{\widetilde{\mathcal{D}}_{\mathfrak{X}}}\left(f_{T,+}\left(\mathcal{M}^{\prime}\right), \widetilde{\omega}_{\mathfrak{X}} \otimes_{\widetilde{\mathcal{O}}_{\mathfrak{X}}} \mathcal{E}\right) .
$$

Lorsque $f$ est un isomorphisme, on notera $\operatorname{adj}_{\widetilde{\omega}_{\mathfrak{X}} \otimes_{\widetilde{\mathcal{O}}_{\mathfrak{X}}} \mathcal{E}}: f_{T+} f_{T}^{!}\left(\widetilde{\omega}_{\mathfrak{X}} \otimes_{\widetilde{\mathcal{O}}_{\mathfrak{X}}}\right.$ $\mathcal{E}) \rightarrow \widetilde{\omega}_{\mathfrak{X}} \otimes_{\widetilde{\mathcal{O}}_{\mathfrak{X}}} \mathcal{E}$, l'image de l'identité de $f_{T}^{!}\left(\widetilde{\omega}_{\mathfrak{X}} \otimes_{\widetilde{\mathcal{O}}_{\mathfrak{X}}} \mathcal{E}\right)$ par 3.2.4.2. Comme $f_{T+} f_{T}^{!}\left(\widetilde{\omega}_{\mathfrak{X}} \otimes_{\widetilde{\mathcal{O}}_{\mathfrak{X}}} \mathcal{E}\right) \stackrel{\sim}{\longrightarrow} \widetilde{\omega}_{\mathfrak{X}} \otimes_{\widetilde{\mathcal{O}}_{\mathfrak{X}}} f_{T+} f_{T}^{!}(\mathcal{E})$ (voir 3.1.7.1 et 3.1.7.2), on obtient le morphisme d'adjonction $\operatorname{adj}_{\mathcal{E}}: f_{T+} f_{T}^{!}(\mathcal{E}) \rightarrow \mathcal{E}$.

Lemme 3.2.5. - Pour tout $\mathcal{E} \in D_{\mathrm{parf}}\left({ }^{g} \mathcal{D}_{\mathfrak{X}, \mathbb{Q}}^{\dagger}\left({ }^{\dagger} T\right)\right)$, le morphisme composé

$f_{T+} f_{T}^{!}\left(\widetilde{\omega}_{\mathfrak{X}} \otimes_{\widetilde{\mathcal{O}}_{\mathfrak{X}}} \mathcal{E}\right) \stackrel{\sim}{\longrightarrow} f_{*}\left(\widetilde{\omega}_{\mathfrak{X}^{\prime}} \otimes_{\widetilde{\mathcal{O}}_{\mathfrak{X}^{\prime}}} f_{T}^{!}(\mathcal{E})\right) \underset{\operatorname{proj}^{\prime}}{\stackrel{\sim}{\sim}} f_{*}\left(\widetilde{\omega}_{\mathfrak{X}^{\prime}}\right) \otimes_{\widetilde{\mathcal{O}}_{\mathfrak{X}}} \mathcal{E} \underset{\operatorname{Tr}}{\sim} \widetilde{\omega}_{\mathfrak{X}} \otimes_{\widetilde{\mathcal{O}}_{\mathfrak{X}}} \mathcal{E}$, où le premier isomorphisme est 3.1.7.1, est égal à $\operatorname{adj}_{\widetilde{\omega}_{\mathfrak{X}} \otimes_{\widetilde{\mathcal{O}}_{\mathfrak{X}}}}$.

Démonstration. - Pour tous $\mathcal{M}^{\prime} \in D_{\text {parf }}\left(\mathcal{D}_{\mathfrak{X}^{\prime}, \mathbb{Q}}^{\dagger}\left({ }^{\dagger} T^{\prime}\right)^{\mathrm{d}}\right), \mathcal{E} \in D_{\text {parf }}\left({ }^{\mathrm{g}} \mathcal{D}_{\mathfrak{X}, \mathbb{Q}}^{\dagger}\left({ }^{\dagger} T\right)\right)$, le diagramme ci-après

$$
\begin{aligned}
& f_{*} \mathbb{R} \mathcal{H o m}_{\widetilde{\mathcal{D}}_{\mathfrak{X}^{\prime}}}\left(\mathcal{M}^{\prime}, f_{T}^{!}\left(\widetilde{\omega}_{\mathfrak{X}} \otimes_{\widetilde{\mathcal{O}}_{\mathfrak{X}}} \mathcal{E}\right)\right) \underset{\sim}{f_{*}} \underset{R}{\sim} \mathcal{H o m}_{\widetilde{\mathcal{D}}_{\mathfrak{X}}}\left(f_{*} \mathcal{M}^{\prime}, f_{*} f_{T}^{!}\left(\widetilde{\omega}_{\mathfrak{X}} \otimes_{\widetilde{\mathcal{O}}_{\mathfrak{X}}} \mathcal{E}\right)\right) \\
& \sim \downarrow \quad \sim \downarrow \\
& f_{*} \mathbb{R} \mathcal{H}_{o m_{\widetilde{\mathcal{D}}_{\mathfrak{X}^{\prime}}}}\left(\mathcal{M}^{\prime}, \widetilde{\omega}_{\mathfrak{X}^{\prime}} \otimes_{\widetilde{\mathcal{O}}_{\mathfrak{X}^{\prime}}} f_{T}^{!}(\mathcal{E})\right) \stackrel{f_{*}}{\sim} \mathbb{R} \mathcal{H}_{\widetilde{\mathcal{D}}_{\mathfrak{X}}}\left(f_{*} \mathcal{M}^{\prime}, f_{*}\left(\widetilde{\omega}_{\mathfrak{X}^{\prime}} \otimes_{\widetilde{\mathcal{O}}_{\mathfrak{X}^{\prime}}} f_{T}^{!}(\mathcal{E})\right)\right) \\
& \operatorname{proj}_{f} \uparrow \sim \\
& \left(f_{*} \mathbb{R} \mathcal{H o m}_{\widetilde{\mathcal{D}}_{\mathfrak{X}^{\prime}}}\left(\mathcal{M}^{\prime}, \widetilde{\omega}_{\mathfrak{X}^{\prime}} \otimes_{\widetilde{\mathcal{O}}_{\mathfrak{X}^{\prime}}} \widetilde{\mathcal{D}}_{\mathfrak{X}^{\prime}}\right)\right) \otimes \widetilde{\mathcal{D}}_{\mathfrak{X}}^{\mathbb{L}} \mathcal{E} \\
& \sim \downarrow \\
& \mathbb{R H o m}_{\widetilde{\mathcal{D}}_{\mathfrak{X}}}\left(f_{*} \mathcal{M}^{\prime}, f_{*}\left(\widetilde{\omega}_{\mathfrak{X}^{\prime}} \otimes_{\widetilde{\mathcal{O}}_{\mathfrak{X}^{\prime}}} \widetilde{\mathcal{D}}_{\mathfrak{X}^{\prime}}\right)\right) \otimes{\widetilde{\widetilde{\mathcal{D}}_{\mathfrak{X}}}}_{\mathbb{L}} \mathcal{E} \\
& \operatorname{proj}^{\prime} \uparrow \sim \\
& \mathbb{R} \mathcal{H o m}_{\widetilde{\mathcal{D}}_{\mathfrak{X}}}\left(f_{*} \mathcal{M}^{\prime}, f_{*}\left(\widetilde{\omega}_{\mathfrak{X}^{\prime}}\right) \otimes_{\widetilde{\mathcal{O}}_{\mathfrak{X}}} \widetilde{\mathcal{D}}_{\mathfrak{X}}\right) \otimes_{\widetilde{\mathcal{D}}_{\mathfrak{X}}}^{\mathbb{L}} \mathcal{\sim} \underset{\sim}{\stackrel{\operatorname{Tr}}{\longrightarrow}} \mathbb{R} \mathcal{H} o m_{\widetilde{\mathcal{D}}_{\mathfrak{X}}}\left(f_{*} \mathcal{M}^{\prime}, \widetilde{\omega}_{\mathfrak{X}} \otimes_{\widetilde{\mathcal{O}}_{\mathfrak{X}}} \mathcal{E}\right), \\
& \operatorname{proj}^{\prime} \uparrow \sim
\end{aligned}
$$

où le morphisme de projection $\operatorname{proj}_{f}$ en haut à droite est l'isomorphisme de la troisième ligne de 3.2.4.1 et dont les deux isomorphismes proj' sont induits par 3.2.2.1, est commutatif. En effet, cela est local en $\mathfrak{X}$. On peut donc supposer que $\mathcal{M}^{\prime}$ possède une résolution bornée par des $\widetilde{\mathcal{D}}_{\mathfrak{X}^{\prime}}$-modules localement projectifs et de type fini. En résolvant platement $\mathcal{E}$, la commutativité du rectangle du bas de 3.2.5.2 résulte d'un calcul. Enfin, celle du carré se vérifie par fonctorialité. 
Or, grâce à 3.2.3.1 (et via l'identification $f_{*}=f_{T+}$ ), la flèche

$$
\left(f_{*} \mathbb{R} \mathcal{H o m}_{\widetilde{\mathcal{D}}_{\mathfrak{X}^{\prime}}}\left(\mathcal{M}^{\prime}, \widetilde{\omega}_{\mathfrak{X}^{\prime}} \otimes_{\widetilde{\mathcal{O}}_{\mathfrak{X}^{\prime}}} \widetilde{\mathcal{D}}_{\mathfrak{X}^{\prime}}\right)\right) \otimes_{\widetilde{\mathcal{D}}_{\mathfrak{X}}}^{\mathbb{L}} \mathcal{E} \stackrel{\sim}{\longrightarrow} \mathcal{H o m}_{\widetilde{\mathcal{D}}_{\mathfrak{X}}}\left(f_{*} \mathcal{M}^{\prime}, \widetilde{\omega}_{\mathfrak{X}} \otimes_{\widetilde{\mathcal{O}}_{\mathfrak{X}}} \mathcal{E}\right)
$$

de 3.2.5.2 (passant par la gauche puis par le bas) est $\otimes \circ \chi$ de 3.2.4.1 (qui s'identifie d'ailleurs à $\otimes \circ \chi \circ \otimes)$. Il en résulte que l'isomorphisme

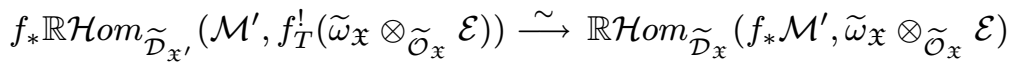

passant par la gauche puis par le bas de 3.2.5.2 est le composé de 3.2.4.1. Pour $\mathcal{M}^{\prime}=f_{T}^{!}\left(\widetilde{\omega}_{\mathfrak{X}} \otimes_{\widetilde{\mathcal{O}}_{\mathfrak{X}}} \mathcal{E}\right)$, en appliquant $H^{0} \circ \mathbb{R} \Gamma(\mathfrak{X},-)$ à 3.2.5.2, il en dérive le diagramme commutatif

$$
\begin{aligned}
& \operatorname{Hom}_{\widetilde{\mathcal{D}}_{\mathfrak{X}^{\prime}}}\left(f_{T}^{!}\left(\widetilde{\omega}_{\mathfrak{X}} \otimes_{\widetilde{\mathcal{O}}_{\mathfrak{X}}} \mathcal{E}\right), f_{T}^{!}\left(\widetilde{\omega}_{\mathfrak{X}} \otimes_{\widetilde{\mathcal{O}}_{\mathfrak{X}}} \mathcal{E}\right)\right) \underset{\sim}{\sim} \operatorname{Hom}_{\widetilde{\mathcal{D}}_{\mathfrak{X}}}\left(f_{T+} f_{T}^{!}\left(\widetilde{\omega}_{\mathfrak{X}} \otimes_{\widetilde{\mathcal{O}}_{\mathfrak{X}}} \mathcal{E}\right), f_{T+} f_{T}^{!}\left(\widetilde{\omega}_{\mathfrak{X}} \otimes_{\widetilde{\mathcal{O}}_{\mathfrak{X}}} \mathcal{E}\right)\right) \\
& \underset{\operatorname{adj}}{\sim \operatorname{Hom}_{\widetilde{\mathcal{D}}_{\mathfrak{X}}}}\left(f_{T+} f_{T}^{!}\left(\widetilde{\omega}_{\mathfrak{X}} \otimes_{\widetilde{\mathcal{O}}_{\mathfrak{X}}} \mathcal{E}\right), \widetilde{\omega}_{\mathfrak{X}} \otimes_{\widetilde{\mathcal{O}}_{\mathfrak{X}}} \mathcal{E}\right)
\end{aligned}
$$

dont le morphisme de droite se déduit du composé de droite de 3.2.5.2 (qui découle fonctoriellement de 3.2.5.1). On conclut en calculant l'image de l'identité $\operatorname{de} f_{T}^{!}\left(\widetilde{\omega}_{\mathfrak{X}} \otimes_{\widetilde{\mathcal{O}}_{\mathfrak{X}}} \mathcal{E}\right)$ via les deux chemins de 3.2.5.3.

3.2.6. - Soit $\mathcal{F}$ un $\widetilde{\mathcal{D}}_{\mathfrak{X}}$-bimodule à gauche. On obtient le $\left(f^{-1} \widetilde{\mathcal{D}}_{\mathfrak{X}}, \widetilde{\mathcal{D}}_{\mathfrak{X}^{\prime}}\right)$ bimodule $f_{T, \mathrm{~d}}^{!}(\mathcal{F})$. Via l'isomorphisme canonique $\iota: f^{-1} \widetilde{\mathcal{D}}_{\mathfrak{X}} \stackrel{\sim}{\longrightarrow} \widetilde{\mathcal{D}}_{\mathfrak{X}^{\prime}}, f_{T, \mathrm{~d}}^{!}(\mathcal{F})$ est ainsi muni d'une structure canonique de $\widetilde{\mathcal{D}}_{\mathfrak{X}^{\prime}}$-bimodule à gauche. De même, en remplaçant « à gauche » par « à droite» ou le symbole « $d »$ par « $g$ » ou en passant aux complexes.

Pour tous $\mathcal{E} \in D\left({ }^{\mathrm{g}} \mathcal{D}_{\mathfrak{X}, \mathbb{Q}}^{\dagger}\left({ }^{\dagger} T\right)\right)$ et $\mathcal{F} \in D^{+}\left({ }^{\mathrm{g}} \mathcal{D}_{\mathfrak{X}, \mathbb{Q}}^{\dagger}\left({ }^{\dagger} T\right),{ }^{\mathrm{g}} \mathcal{D}_{\mathfrak{X}, \mathbb{Q}}^{\dagger}\left({ }^{\dagger} T\right)\right)$, on obtient ainsi les deuxièmes isomorphisme des composés :

$f_{T}^{!} \mathbb{R H o m}_{\widetilde{\mathcal{D}}_{\mathfrak{X}}}(\mathcal{E}, \mathcal{F}) \stackrel{\sim}{\longrightarrow} \mathbb{R} \mathcal{H}_{f^{-1}} \widetilde{\mathcal{D}}_{\mathfrak{X}}\left(f^{-1} \mathcal{E}, f_{T, \mathrm{~d}}^{!} \mathcal{F}\right) \stackrel{\sim}{\longrightarrow} \mathbb{R} \mathcal{H}_{\widetilde{\mathcal{D}}_{\mathfrak{X}^{\prime}}}\left(f_{T}^{!}(\mathcal{E}), f_{T, \mathrm{~d}}^{!}(\mathcal{F})\right)$,

$f_{T, \mathrm{~d}}^{!} \mathbb{R} \mathcal{H o m}_{\widetilde{\mathcal{O}}_{\mathfrak{X}}}(\mathcal{E}, \mathcal{F}) \stackrel{\sim}{\longrightarrow} \mathbb{R} \mathcal{H o m}_{f^{-1} \widetilde{\mathcal{O}}_{\mathfrak{X}}}\left(f^{-1} \mathcal{E}, f_{T, \mathrm{~d}}^{!} \mathcal{F}\right) \stackrel{\sim}{\longrightarrow} \mathbb{R} \mathcal{H o m}_{\widetilde{\mathcal{O}}_{\mathfrak{X}^{\prime}}}\left(f_{T}^{!}(\mathcal{E}), f_{T, \mathrm{~d}}^{!}(\mathcal{F})\right)$.

De même, pour tous $\mathcal{E} \in D^{-}\left({ }^{\mathrm{g}} \mathcal{D}_{\mathfrak{X}, \mathbb{Q}}^{\dagger}\left({ }^{\dagger} T\right)\right), \mathcal{F} \in D^{-}\left({ }^{\mathrm{g}} \mathcal{D}_{\mathfrak{X}, \mathbb{Q}}^{\dagger}\left({ }^{\dagger} T\right),{ }^{\mathrm{g}} \mathcal{D}_{\mathfrak{X}, \mathbb{Q}}^{\dagger}\left({ }^{\dagger} T\right)\right)$,

$$
f_{T, \mathrm{~d}}^{!}\left(\mathcal{E} \otimes \widetilde{\mathcal{O}}_{\mathfrak{X}}^{\mathbb{L}} \mathcal{F}\right) \stackrel{\sim}{\longrightarrow} f^{-1}(\mathcal{E}) \otimes_{f^{-1} \widetilde{\mathcal{O}}_{\mathfrak{X}}}^{\mathbb{L}} f_{T, \mathrm{~d}}^{!}(\mathcal{F}) \stackrel{\sim}{\longrightarrow} f_{T}^{!}(\mathcal{E}) \otimes_{\widetilde{\mathcal{O}}_{\mathfrak{X}^{\prime}}}^{\mathbb{L}} f_{T, \mathrm{~d}}^{!}(\mathcal{F})
$$

On remarque que la structure gauche de $\mathcal{E} \otimes \widetilde{\mathcal{O}}_{\mathfrak{X}}^{\mathbb{L}} \mathcal{F}$ étant celle du produit tensoriel, $f_{T, \mathrm{~g}}^{!}\left(\mathcal{E} \otimes \widetilde{\mathcal{O}}_{\mathfrak{x}}^{\mathbb{L}} \mathcal{F}\right) \stackrel{\sim}{\longrightarrow} f_{T}^{!}(\mathcal{E}) \otimes \mathbb{\widetilde { \mathcal { O } }}_{\mathfrak{x}^{\prime}} f_{T, \mathrm{~g}}^{!}(\mathcal{F})$ (pour ce dernier isomorphisme, l'hypothèse que $f$ est un isomorphisme est superflu, mais il faut ajouté le décalage $\left.\left[d_{X^{\prime} / X}\right]\right)$. 
3.2.7. - Soit $\mathcal{E} \in D_{\text {parf }}\left({ }^{g} \mathcal{D}_{\mathfrak{X}, \mathbb{Q}}^{\dagger}\left({ }^{\dagger} T\right)\right)$. On note $\xi: f_{T}^{!} \mathbb{D}_{T}^{\prime}(\mathcal{E}) \stackrel{\sim}{\longrightarrow} \mathbb{D}_{T^{\prime}}^{\prime} f_{T}^{!}(\mathcal{E})$ l'isomorphisme défini comme suit

$$
\begin{aligned}
\xi: f_{T}^{!} & \mathbb{D}_{T}^{\prime}(\mathcal{E}) \stackrel{\sim}{\longrightarrow} \mathbb{R} \mathcal{H o m}_{\widetilde{\mathcal{D}}_{\mathfrak{X}^{\prime}}}\left(f_{T}^{!}(\mathcal{E}), f_{T, \mathrm{~d}}^{!}\left(\left(\widetilde{\mathcal{D}}_{\mathfrak{X}} \otimes_{\widetilde{\mathcal{O}}_{\mathfrak{X}}} \widetilde{\omega}_{\mathfrak{X}}^{-1}\right)_{\mathrm{t}}\right)\right)\left[d_{X}\right] \\
& \stackrel{\sim}{\longrightarrow} \mathbb{R} \mathcal{H o m}_{\widetilde{\mathcal{D}}_{\mathfrak{X}^{\prime}}}\left(f_{T}^{!} \mathcal{E},\left(\widetilde{\mathcal{D}}_{\mathfrak{X}^{\prime}} \otimes_{\widetilde{\mathcal{O}}_{\mathfrak{X}^{\prime}}} \widetilde{\omega}_{\mathfrak{X}^{\prime}}^{-1}\right)_{\mathrm{t}}\right)\left[d_{X^{\prime}}\right]=\mathbb{D}_{T^{\prime}}^{\prime} f_{T}^{!}(\mathcal{E}),
\end{aligned}
$$

où l'isomorphisme de la première ligne (resp. deuxième ligne) est 3.2.6.1 (resp. 3.1.8). Il en découle l'isomorphisme : $f_{T}^{!} \mathbb{D}_{T}(\mathcal{E}) \underset{\beta}{\stackrel{\sim}{\longrightarrow}} f_{T}^{!} \mathbb{D}_{T}^{\prime}(\mathcal{E}) \underset{\xi}{\stackrel{\sim}{\longrightarrow}}$ $\mathbb{D}_{T^{\prime}}^{\prime} f_{T}^{!}(\mathcal{E}) \underset{\beta}{\stackrel{\sim}{\longrightarrow}} \mathbb{D}_{T^{\prime}} f_{T}^{!}(\mathcal{E})$, que l'on notera à nouveau $\xi$.

Grâce à 3.1.7.1 et 3.2.1.4, on obtient le composé :

$f_{T}^{!} \circ \mathbb{D}_{T}\left(\widetilde{\omega}_{\mathfrak{X}} \otimes_{\widetilde{\mathcal{O}}_{\mathfrak{X}}} \mathcal{E}\right) \stackrel{\sim}{\longrightarrow} f_{T}^{!}\left(\widetilde{\omega}_{\mathfrak{X}} \otimes_{\widetilde{\mathcal{O}}_{\mathfrak{X}}} \mathbb{D}_{T}^{\prime}(\mathcal{E})\right) \stackrel{\sim}{\longrightarrow} \widetilde{\omega}_{\mathfrak{X}^{\prime}} \otimes_{\widetilde{\mathcal{O}}_{\mathfrak{X}^{\prime}}} f_{T}^{!}\left(\mathbb{D}_{T}^{\prime}(\mathcal{E})\right) \stackrel{\xi}{\longrightarrow} \widetilde{\omega}_{\mathfrak{X}^{\prime}} \otimes_{\widetilde{\mathcal{O}}_{\mathfrak{X}^{\prime}}} \mathbb{D}_{T^{\prime}}^{\prime}\left(f_{T}^{!}(\mathcal{E})\right)$

$$
\stackrel{\sim}{\longrightarrow} \mathbb{D}_{T^{\prime}}\left(\widetilde{\omega}_{\mathfrak{X}^{\prime}} \otimes_{\widetilde{\mathcal{O}}_{\mathfrak{X}^{\prime}}} f_{T}^{!}(\mathcal{E})\right) \stackrel{\sim}{\longrightarrow} \mathbb{D}_{T^{\prime}} \circ f_{T}^{!}\left(\widetilde{\omega}_{\mathfrak{X}} \otimes_{\widetilde{\mathcal{O}}_{\mathfrak{X}}} \mathcal{E}\right)
$$

encore noté $\xi$. Pour tout $\mathcal{M} \in D_{\text {parf }}\left(\mathcal{D}_{\mathfrak{X}, \mathbb{Q}}^{\dagger}\left({ }^{\dagger} T\right)^{\mathrm{d}}\right)$, on a ainsi l'isomorphisme $\xi$ : $f_{T}^{!} \mathbb{D}_{T}(\mathcal{M}) \stackrel{\sim}{\longrightarrow} \mathbb{D}_{T^{\prime}} f_{T}^{!}(\mathcal{M})$.

REMARQUES 3.2.8. - Grâce au diagramme de droite de 3.1.8.2, avec les notations 3.2 .7 , on aurait pu définir $\xi$ comme suit :

$$
\begin{aligned}
\xi: & f_{T}^{!} \mathbb{D}_{T}(\mathcal{E}) \stackrel{\sim}{\longrightarrow} \mathbb{R} \mathcal{H o m} \widetilde{\mathcal{D}}_{\mathfrak{X}^{\prime}}\left(f_{T}^{!}(\mathcal{E}), f_{T, \mathrm{~d}}^{!}\left(\widetilde{\mathcal{D}}_{\mathfrak{X}} \otimes_{\widetilde{\mathcal{O}}_{\mathfrak{X}}} \widetilde{\omega}_{\mathfrak{X}}^{-1}\right)\right)\left[d_{X}\right] \\
& \left.\stackrel{\sim}{\longrightarrow} \mathbb{R} \mathcal{H o m}_{\widetilde{\mathcal{D}}_{\mathfrak{X}^{\prime}}}\left(f_{T}^{!}(\mathcal{E}), \widetilde{\mathcal{D}}_{\mathfrak{X}^{\prime}} \otimes_{\widetilde{\mathcal{O}}_{\mathfrak{X}^{\prime}}} \widetilde{\omega}_{\mathfrak{X}^{\prime}}^{-1}\right)_{\mathrm{t}}\right)\left[d_{X}\right] \underset{\beta}{\stackrel{\sim}{\longrightarrow}} \mathbb{D}_{T^{\prime}} f_{T}^{!}(\mathcal{E}) .
\end{aligned}
$$

Avec la remarque de 3.1.8, on vérifie que lorsque $f$ est l'identité $\xi$ est l'identité.

Proposition 3.2.9. - Soit $\mathcal{M} \in D_{\text {parf }}\left({ }^{*} \mathcal{D}_{\mathfrak{X}, \mathbb{Q}}^{\dagger}\left({ }^{\dagger} T\right)\right)$. Le diagramme

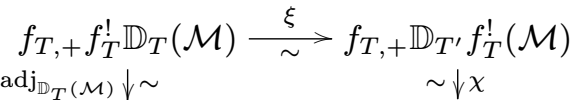

$$
\begin{aligned}
& \mathbb{D}_{T}(\mathcal{M}) \underset{\mathbb{D}_{T} \operatorname{adj}_{\mathcal{M}}}{\sim} \mathbb{D}_{T} f_{T,+} f_{T}^{!}(\mathcal{M})
\end{aligned}
$$

est commutatif. De même, en remplaçant $\mathbb{D}$ par $\mathbb{D}^{\prime}$. 
Démonstration. - Comme les carrés de droite, de gauche, du haut et du bas du diagramme

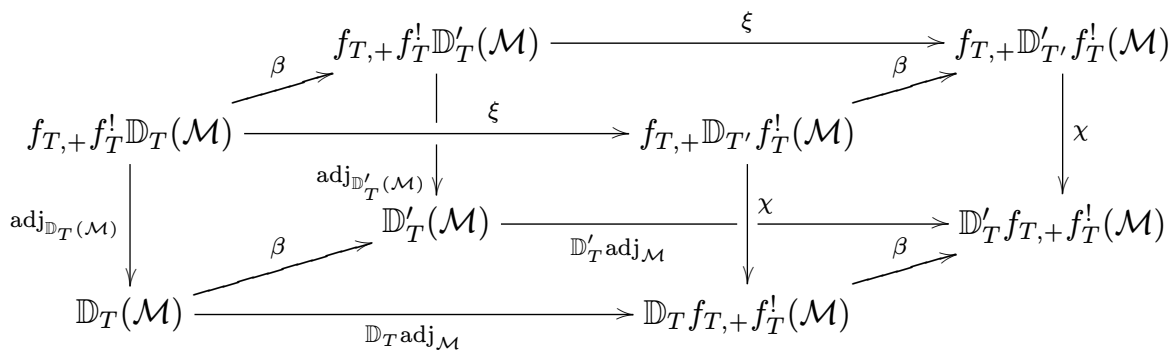

sont commutatif, il suffit de prouver la proposition pour $\mathbb{D}^{\prime}$. De même, grâce à $3.1 .7 .1,3.1 .7 .2,3.2 .1 .3$ et 3.2 .1 .4 , on vérifie que le cas où $*=g$ se déduit du cas $*=d$. Traitons donc ce dernier. Pour tout $\mathcal{F} \in D_{\text {parf }}\left({ }^{\mathrm{g}} \mathcal{D}_{\mathfrak{X}, \mathbb{Q}}^{\dagger}\left({ }^{\dagger} T\right)\right)$, notons $\alpha$ le morphisme composé $f_{*}\left(\widetilde{\omega}_{\mathfrak{X}^{\prime}} \otimes_{\widetilde{\mathcal{O}}_{\mathfrak{X}^{\prime}}} f_{T}^{!}(\mathcal{F})\right) \underset{\text { proj' }}{\stackrel{\sim}{\sim}} f_{*}\left(\widetilde{\omega}_{\mathfrak{X}^{\prime}}\right) \otimes_{\widetilde{\mathcal{O}}_{\mathfrak{X}}} \mathcal{F} \underset{\operatorname{Tr}}{\stackrel{\sim}{\longrightarrow}} \widetilde{\omega}_{\mathfrak{X}} \otimes_{\widetilde{\mathcal{O}}_{\mathfrak{X}}} \mathcal{F}$, où $\operatorname{proj}^{\prime}$ a été défini en 3.2.2.1.

Soit $\mathcal{E} \in D_{\text {parf }}\left({ }^{g} \mathcal{D}_{\mathfrak{X}, \mathbb{Q}}^{\dagger}\left({ }^{\dagger} T\right)\right)$ tel que $\mathcal{M}=\widetilde{\omega}_{\mathfrak{X}} \otimes_{\widetilde{\mathcal{O}}_{\mathfrak{X}}} \mathcal{E}$. D'après 3.2.5, par fonctorialité en l'isomorphisme $\mathbb{D}_{T}^{\prime}\left(\widetilde{\omega}_{\mathfrak{X}} \otimes_{\widetilde{\mathcal{O}}_{\mathfrak{X}}} \mathcal{E}\right) \stackrel{\sim}{\longrightarrow} \widetilde{\omega}_{\mathfrak{X}} \otimes_{\widetilde{\mathcal{O}}_{\mathfrak{X}}} \mathbb{D}_{T}(\mathcal{E})$, le composé de gauche du diagramme ci-dessous

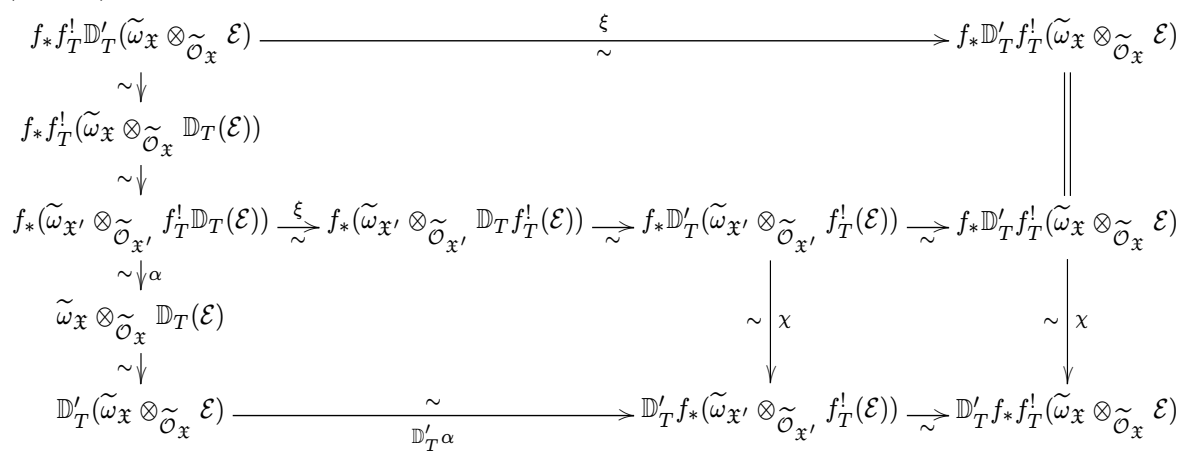

est égal à $\operatorname{adj}_{\mathbb{D}_{T}^{\prime}\left(\widetilde{\omega}_{\mathfrak{X}} \otimes_{\widetilde{\mathcal{O}}_{\mathfrak{X}}} \mathcal{E}\right)}$. Avec 3.2 .5 , le composé du bas est $\mathbb{D}_{T}^{\prime} \operatorname{adj}_{\widetilde{\omega}_{\mathfrak{X}} \otimes_{\widetilde{\mathcal{O}}_{\mathfrak{X}}} \mathcal{E}}$. Ainsi, le contour de 3.2.9.3 correspond à celui de 3.2.9.1. Le rectangle du haut est commutatif par définition (3.2.7.2), celui en bas à droite l'est par fonctorialité. Enfin, via 3.2.3.1 et 3.2.8.1, on établit par un calcul local (où $f_{*}$ est le foncteur oubli et où on identifie $\widetilde{\omega}_{\mathfrak{X}}$ à $\widetilde{\mathcal{O}}_{\mathfrak{X}}$ grâce à la base $d t_{1} \wedge \cdots \wedge d t_{d}$ etc.) celle du rectangle en bas à gauche. 
REMARQUes 3.2.10. - Avec les notations de 3.2.9 et dans le cas à gauche, il résulte de 3.2.9 et de 4.2.2.2 que l'isomorphisme $\xi$ est égal à l'isomorphisme $\theta_{f, T, \mathcal{M}}$ défini en 4.2.1.

\subsection{Preuve de la compatibilité aux images inverses par une immersion ouverte}

3.3.1 (Convention). - On pose ici $\widetilde{\mathcal{O}}_{\mathfrak{X}}:=\mathcal{O}_{\mathfrak{X}}\left({ }^{\dagger} T\right)_{\mathbb{Q}} \widetilde{\omega}_{\mathfrak{X}}:=\omega_{\mathfrak{X}} \otimes_{\mathcal{O}_{\mathfrak{X}}} \widetilde{\mathcal{O}}_{\mathfrak{X}}, \widetilde{\mathcal{D}}_{\mathfrak{X}}:=$ $\mathcal{D}_{\mathfrak{X}}\left({ }^{\dagger} T\right)_{\mathbb{Q}}$. De même en rajoutant des primes.

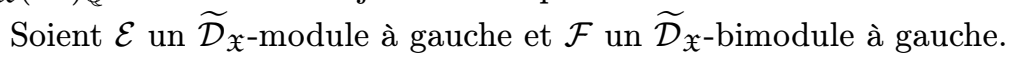

Pour calculer le produit tensoriel $\mathcal{G}_{1}:=\mathcal{F} \otimes_{\widetilde{\mathcal{O}}_{\mathfrak{X}}} \mathcal{E}$, on prendra la structure droite de $\mathcal{F}$. Par fonctorialité, $\mathcal{G}_{1}$ est muni d'une structure de $\widetilde{\mathcal{D}}_{\mathfrak{X}}$-bimodule à gauche. Par convention, la structure gauche de $\mathcal{G}_{1}$ sera celle induite fonctoriellement par la structure gauche de $\mathcal{F}$ et la structure droite sera celle induite par le produit tensoriel.

Lorsque l'on choisira d'inverser ces deux structures, on notera $\mathcal{G}_{2}:=\mathcal{F} \otimes_{\widetilde{\mathcal{O}}_{\mathfrak{X}}}^{\mathrm{t}} \mathcal{E}$. Ainsi, $\mathcal{G}_{2}=\mathcal{G}_{1}$ sauf que la structure droite de $\mathcal{G}_{1}$ est la structure gauche de $\mathcal{G}_{2}$ et vice versa.

Pour calculer $\mathcal{G}_{3}:=\mathcal{E} \otimes_{\widetilde{\mathcal{O}}_{\mathfrak{X}}} \mathcal{F}$, on prendra la structure gauche de $\mathcal{F}$. Par convention, la structure gauche de $\mathcal{G}_{3}$ sera la structure induite par le produit tensoriel et la structure droite sera celle déduite de la structure droite de $\mathcal{F}$.

On définit de même $\mathcal{E} \otimes_{\widetilde{\mathcal{O}}_{\mathfrak{X}}}^{\mathrm{t}} \mathcal{F}$ comme étant le $\widetilde{\mathcal{D}}_{\mathfrak{X}}$-bimodule à gauche égale à $\mathcal{G}_{3}$ modulo un inversement de ses structures gauche et droite.

Afin de prouver la proposition 3.3.9, établissons d'abord les lemmes qui suivent.

LEMme 3.3.2. - Soient $\mathcal{E} \in D^{-}\left({ }^{\mathrm{g}} \widetilde{\mathcal{D}}_{\mathfrak{X}}\right), \mathcal{F} \in D^{+}\left({ }^{\mathrm{g}} \widetilde{\mathcal{D}}_{\mathfrak{X}},{ }^{\mathrm{g}} \widetilde{\mathcal{D}}_{\mathfrak{X}}\right)$ et $\mathcal{G} \in$ $D_{\text {tdf }}\left({ }^{g} \widetilde{\mathcal{D}}_{\mathfrak{X}}\right)$. Le diagramme

$$
\begin{aligned}
& f_{T}^{!}\left(\mathbb{R} \mathcal{H} m_{\widetilde{\mathcal{D}}_{\mathfrak{X}}}(\mathcal{E}, \mathcal{F}) \otimes_{\widetilde{\mathcal{O}}_{\mathfrak{X}}}^{\mathbb{L}} \mathcal{G}\right) \longrightarrow f_{T}^{!}\left(\mathbb{R} \mathcal{H} \text { om } \widetilde{\mathcal{D}}_{\mathfrak{X}}\left(\mathcal{E}, \mathcal{F} \otimes_{\widetilde{\mathcal{O}}_{\mathfrak{X}}}^{\mathbb{L}} \mathcal{G}\right)\right) \\
& \sim \downarrow \\
& f_{T}^{!}\left(\mathbb{R} \mathcal{H} o m_{\widetilde{\mathcal{D}}_{\mathfrak{X}}}(\mathcal{E}, \mathcal{F})\right) \otimes_{\widetilde{\mathcal{O}}_{\mathfrak{X}^{\prime}}}^{\mathbb{L}} f_{T}^{!}(\mathcal{G})
\end{aligned}
$$

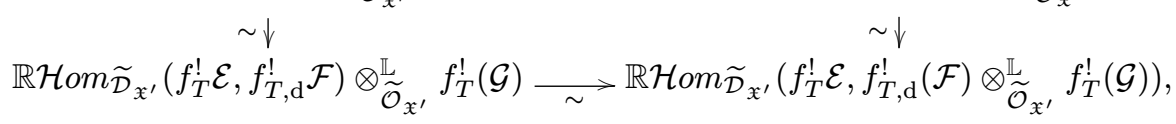

dont les isomorphismes $\widetilde{\mathcal{D}}_{\mathfrak{X}}$-linéaires horizontaux sont les isomorphismes $([10$, 2.1.26]) et dont deux des flèches verticales sont 3.2.6.1, est commutatif.

Démonstration. — On résout $\mathcal{F}$ injectivement et $\mathcal{G}$ platement. La commutativité de 3.3.2.1 découle alors d'un calcul local. 
Lemme 3.3.3. - Soient $\mathcal{E} \in D^{-}\left({ }^{\mathrm{g}} \widetilde{\mathcal{D}}_{\mathfrak{X}}\right), \mathcal{F} \in D^{-}\left({ }^{\mathrm{g}} \widetilde{\mathcal{D}}_{\mathfrak{X}}\right)$ et $\mathcal{G} \in D^{+}\left({ }^{\mathrm{g}} \widetilde{\mathcal{D}}_{\mathfrak{X}},{ }^{*} \widetilde{\mathcal{D}}_{\mathfrak{X}}\right)$. Le diagramme

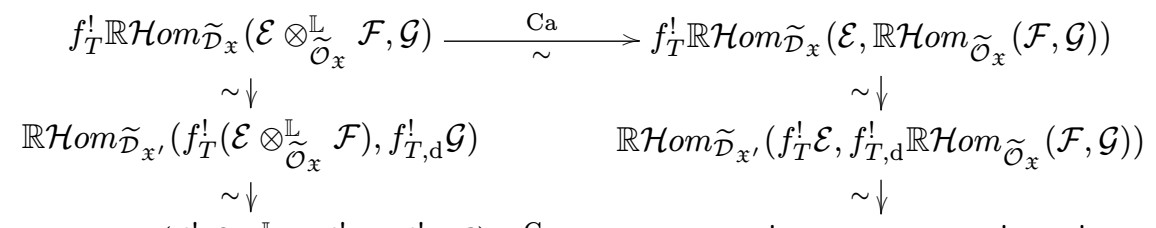

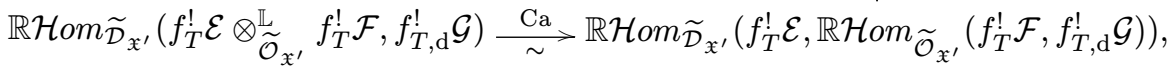

où les isomorphismes $\widetilde{\mathcal{D}}_{\mathfrak{X}}$-linéaires horizontaux sont les isomorphismes de Cartan (10, 2.1.34]), où les isomorphismes verticaux du haut (resp. en bas à droite) découlent de 3.2.6.1 (resp. 3.2.6.2), est commutatif.

Démonstration. - On résout $\mathcal{F}$ par $\widetilde{\mathcal{D}}_{\mathfrak{X}}$-modules à gauche plat et $\mathcal{G}$ par des $\widetilde{\mathcal{D}}_{\mathfrak{X}}$-bimodules à gauche injectifs.

LEMme 3.3.4. - Soit $\mathcal{E}$ un $\widetilde{\mathcal{D}}_{\mathfrak{X}}$-module à gauche. Avec les notations de 3.3.1, on dispose du diagramme commutatif :

$$
\begin{aligned}
& \left(\widetilde{\mathcal{D}}_{\mathfrak{X}} \otimes_{\widetilde{\mathcal{O}}_{\mathfrak{X}}} \widetilde{\omega}_{\mathfrak{X}}^{-1}\right) \otimes_{\widetilde{\mathcal{O}}_{\mathfrak{X}}} \mathcal{E} \underset{\sim}{\gamma_{\mathcal{E}} \otimes \omega^{-1}} \mathcal{\sim} \mathcal{E} \otimes_{\widetilde{\mathcal{O}}_{\mathfrak{X}}}\left(\widetilde{\mathcal{D}}_{\mathfrak{X}} \otimes_{\widetilde{\mathcal{O}}_{\mathfrak{X}}} \widetilde{\omega}_{\mathfrak{X}}^{-1}\right) \\
& \sim \downarrow \beta \quad \sim \downarrow \beta \\
& \left(\widetilde{\mathcal{D}}_{\mathfrak{X}} \otimes_{\widetilde{\mathcal{O}}_{\mathfrak{X}}} \widetilde{\omega}_{\mathfrak{X}}^{-1}\right)_{\mathrm{t}} \otimes_{\widetilde{\mathcal{O}}_{\mathfrak{X}}} \mathcal{E} \quad \mathcal{E} \otimes_{\widetilde{\mathcal{O}}_{\mathfrak{X}}}\left(\widetilde{\mathcal{D}}_{\mathfrak{X}} \otimes_{\widetilde{\mathcal{O}}_{\mathfrak{X}}} \widetilde{\omega}_{\mathfrak{X}}^{-1}\right)_{\mathrm{t}} \\
& \mathcal{E} \otimes_{\widetilde{\mathcal{O}}_{\mathfrak{X}}}^{\mathrm{t}}\left(\widetilde{\mathcal{D}}_{\mathfrak{X}}^{\|} \otimes_{\widetilde{\mathcal{O}}_{\mathfrak{X}}} \widetilde{\omega}_{\mathfrak{X}}^{-1}\right) \stackrel{\gamma_{\mathcal{E}} \otimes \omega^{-1}}{\sim}\left(\widetilde{\mathcal{D}}_{\mathfrak{X}} \otimes_{\widetilde{\mathcal{O}}_{\mathfrak{X}}} \widetilde{\omega}_{\mathfrak{X}}^{-1}\right) \otimes_{\widetilde{\mathcal{O}}_{\mathfrak{X}}}^{\mathrm{t}} \mathcal{E} .
\end{aligned}
$$

Démonstration. - En utilisant les caractérisations de [4, 1.3.1 et 1.3.3] des isomorphismes de transposition, on calcule que l'image de $(1 \otimes \omega) \otimes e$, avec $e$ (resp. $\omega$ ) une section locale de $\mathcal{E}$ (resp. $\widetilde{\omega}_{\mathfrak{X}}^{-1}$ ), ne dépend pas du chemin suivi. On conclut par $\widetilde{\mathcal{D}}_{\mathfrak{X}}$-linéarité.

Afin d'établir le diagramme commutatif 3.3.7.3, nous aurons besoin des deux lemmes ci-dessous. 
Lemme 3.3.5. - Soit $\mathcal{G} \in D\left({ }^{\mathrm{g}} \widetilde{\mathcal{D}}_{\mathfrak{X}}\right)$. Le diagramme ci-après $(3.3 .5 .1)$

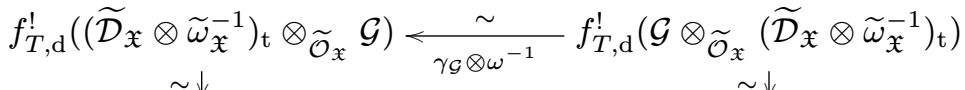

$$
\begin{aligned}
& f_{T, \mathrm{~d}}^{!}\left(\left(\widetilde{\mathcal{D}}_{\mathfrak{X}} \otimes \widetilde{\omega}_{\mathfrak{X}}^{-1}\right)_{\mathrm{t}}\right) \otimes_{\widetilde{\mathcal{O}}_{\mathfrak{X}^{\prime}}} f_{T}^{!}(\mathcal{G}) \quad f_{T}^{!}(\mathcal{G}) \otimes_{\widetilde{\mathcal{O}}_{\mathfrak{X}^{\prime}}} f_{T, \mathrm{~d}}^{!}\left(\left(\widetilde{\mathcal{D}}_{\mathfrak{X}} \otimes \widetilde{\omega}_{\mathfrak{X}}^{-1}\right)_{\mathrm{t}}\right) \\
& \sim \downarrow \quad \sim \downarrow \\
& \left(\widetilde{\mathcal{D}}_{\mathfrak{X}^{\prime}} \otimes \widetilde{\omega}_{\mathfrak{X}^{\prime}}^{-1}\right)_{\mathrm{t}} \otimes \widetilde{\mathcal{O}}_{\mathfrak{X}^{\prime}} f_{T}^{!}(\mathcal{G}) \underset{\gamma_{f_{T}^{!}(\mathcal{G})} \otimes \omega^{-1}}{\sim} f_{T}^{!}(\mathcal{G}) \otimes_{\widetilde{\mathcal{O}}_{\mathfrak{X}^{\prime}}}\left(\widetilde{\mathcal{D}}_{\mathfrak{X}^{\prime}} \otimes \widetilde{\omega}_{\mathfrak{X}^{\prime}}^{-1}\right)_{\mathrm{t}}
\end{aligned}
$$

dont la flèche en haut à droite est 3.2.6.3 et dont les flèches horizontales dérivent fonctoriellement de celle du bas de 3.3.4.1, est commutatif.

Démonstration. - Cela se vérifie par un calcul local.

Lemme 3.3.6. - Soient $\mathcal{E} \in D\left({ }^{8} \widetilde{\mathcal{D}}_{\mathfrak{X}}\right), \mathcal{F} \in D_{\text {tdf,. }}^{+}\left({ }^{\mathrm{g}} \widetilde{\mathcal{D}}_{\mathfrak{X}},{ }^{\mathrm{g}} \widetilde{\mathcal{D}}_{\mathfrak{X}}\right)$. On bénéficie du diagramme commutatif ci-dessous :

$$
\begin{aligned}
& f_{T, \mathrm{~d}}^{!}\left(\mathcal{E}^{\vee} \otimes_{\widetilde{\mathcal{O}}_{\mathfrak{X}}}^{\mathbb{L}} \mathcal{F}\right) \longrightarrow f_{T, \mathrm{~d}}^{!}\left(\mathbb{R} \mathcal{H} \operatorname{Hom}_{\widetilde{\mathcal{O}}_{\mathfrak{X}}}(\mathcal{E}, \mathcal{F})\right) \\
& \sim \downarrow \\
& f_{T}^{!}\left(\mathcal{E}^{\vee}\right) \otimes \widetilde{\mathcal{O}}_{\mathfrak{X}^{\prime}}^{\mathbb{L}} f_{T, \mathrm{~d}}^{!}(\mathcal{F}) \\
& f_{T}^{!}(\mathcal{E})^{\vee} \otimes \widetilde{\mathcal{O}}_{\mathfrak{X}^{\prime}}^{\mathbb{L}} f_{T, \mathrm{~d}}^{!}(\mathcal{F}) \underset{\sim}{\longrightarrow} \mathbb{R} \mathcal{H o m}_{\widetilde{\mathcal{O}}_{\mathfrak{X}}}\left(f_{T}^{!}(\mathcal{E}), f_{T, \mathrm{~d}}^{!}(\mathcal{F})\right),
\end{aligned}
$$

dont la flèche en haut à gauche est 3.2.6.3 et celle de droite est 3.2.6.2.

Démonstration. - On résout $\mathcal{F}$ platement et $\widetilde{\mathcal{O}_{\mathfrak{X}}}$ injectivement puis on conclut via un calcul local immédiat.

3.3.7. - Soit $\mathcal{E} \in D\left({ }^{\mathrm{g}} \widetilde{\mathcal{D}}_{\mathfrak{X}}\right)$. En appliquant 3.3.5.1 à $\mathcal{G}=\mathcal{E}^{\vee}$, on obtient le carré supérieur du diagramme :

$$
\begin{aligned}
& f_{T, \mathrm{~d}}^{!}\left(\left(\widetilde{\mathcal{D}}_{\mathfrak{X}} \otimes \widetilde{\omega}_{\mathfrak{X}}^{-1}\right)_{\mathrm{t}} \otimes_{\widetilde{\mathcal{O}}_{\mathfrak{X}}} \mathcal{E}^{\vee}\right) \underset{\gamma \otimes \omega^{-1}}{\sim} f_{T, \mathrm{~d}}^{!}\left(\mathcal{E}^{\vee} \otimes_{\widetilde{\mathcal{O}}_{\mathfrak{X}}}\left(\widetilde{\mathcal{D}}_{\mathfrak{X}} \otimes \widetilde{\omega}_{\mathfrak{X}}^{-1}\right)_{\mathrm{t}}\right) \\
& \left(\widetilde{\mathcal{D}}_{\mathfrak{X}^{\prime}} \otimes \widetilde{\omega}_{\mathfrak{X}^{\prime}}^{-1}\right)_{\mathrm{t}} \otimes_{\widetilde{\mathcal{O}}_{\mathfrak{X}^{\prime}}} f_{T}^{!}\left(\mathcal{E}^{\vee}\right) \underset{\gamma \otimes \omega^{-1}}{\sim \sim} f_{T}^{!}\left(\mathcal{E}^{\vee}\right) \otimes_{\widetilde{\mathcal{O}}_{\mathfrak{X}^{\prime}}}\left(\widetilde{\mathcal{D}}_{\mathfrak{X}^{\prime}} \otimes \widetilde{\omega}_{\mathfrak{X}^{\prime}}^{-1}\right)_{\mathrm{t}} \\
& \sim \downarrow \quad \gamma \otimes \omega^{-1}, \quad \sim \downarrow \\
& \left(\widetilde{\mathcal{D}}_{\mathfrak{X}^{\prime}} \otimes \widetilde{\omega}_{\mathfrak{X}^{\prime}}^{-1}\right)_{\mathrm{t}} \otimes_{\widetilde{\mathcal{O}}_{\mathfrak{X}^{\prime}}} f_{T}^{!}(\mathcal{E})^{\vee} \underset{\gamma \otimes \omega^{-1}}{\sim} f_{T}^{!}(\mathcal{E})^{\vee} \otimes_{\widetilde{\mathcal{O}}_{\mathfrak{X}^{\prime}}}\left(\widetilde{\mathcal{D}}_{\mathfrak{X}^{\prime}} \otimes \widetilde{\omega}_{\mathfrak{X}^{\prime}}^{-1}\right)_{\mathrm{t}} .
\end{aligned}
$$


Le carré du bas est commutatif par fonctorialité. Le diagramme 3.3.6.1 utilisé pour $\mathcal{F}=\left(\widetilde{\mathcal{D}}_{\mathfrak{X}} \otimes \widetilde{\omega}_{\mathfrak{X}}^{-1}\right)_{\mathrm{t}}$ donne le carré du haut de :

$$
\begin{aligned}
& f_{T, \mathrm{~d}}^{!}\left(\mathcal{E}^{\vee} \otimes \underset{\widetilde{\mathcal{O}}_{\mathfrak{X}}}{\mathbb{L}}\left(\widetilde{\mathcal{D}}_{\mathfrak{X}} \otimes \widetilde{\omega}_{\mathfrak{X}}^{-1}\right)_{\mathrm{t}}\right) \longrightarrow f_{T, \mathrm{~d}}^{!}\left(\mathbb{R} \mathcal{H} \text { om } \widetilde{\mathcal{O}}_{\mathfrak{X}}\left(\mathcal{E},\left(\widetilde{\mathcal{D}}_{\mathfrak{X}} \otimes \widetilde{\omega}_{\mathfrak{X}}^{-1}\right)_{\mathrm{t}}\right)\right) \\
& \sim \downarrow \quad \sim \downarrow \\
& f_{T}^{!}(\mathcal{E})^{\vee} \otimes \widetilde{\mathcal{O}}_{\mathfrak{X}^{\prime}} f_{T, \mathrm{~d}}^{!}\left(\left(\widetilde{\mathcal{D}}_{\mathfrak{X}} \otimes \widetilde{\omega}_{\mathfrak{X}}^{-1}\right)_{\mathrm{t}}\right) \longrightarrow \sim \mathbb{R} \mathcal{H}_{\sim} \widetilde{\mathcal{O}}_{\mathfrak{X}}\left(f_{T}^{!}(\mathcal{E}), f_{T, \mathrm{~d}}^{!}\left(\left(\widetilde{\mathcal{D}}_{\mathfrak{X}} \otimes \widetilde{\omega}_{\mathfrak{X}}^{-1}\right)_{\mathrm{t}}\right)\right)
\end{aligned}
$$

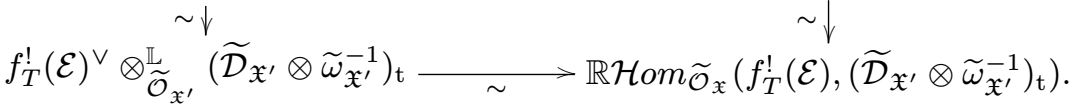

On bénéficie par fonctorialité de la commutativité du carré du bas et donc celle 3.3.7.2. En composant 3.3.7.1 et 3.3.7.2, on obtient ainsi le diagramme commutatif :

$$
\begin{aligned}
& f_{T, \mathrm{~d}}^{!}\left(\left(\widetilde{\mathcal{D}}_{\mathfrak{X}} \otimes \widetilde{\omega}_{\mathfrak{X}}^{-1}\right)_{\mathrm{t}} \otimes \widetilde{\mathcal{O}}_{\mathfrak{X}} \mathcal{E}^{\vee}\right) \longrightarrow f_{T, \mathrm{~d}}^{!}\left(\mathbb{R} \mathcal{H} \text { om }_{\widetilde{\mathcal{O}}_{\mathfrak{X}}}\left(\mathcal{E},\left(\widetilde{\mathcal{D}}_{\mathfrak{X}} \otimes \widetilde{\omega}_{\mathfrak{X}}^{-1}\right)_{\mathrm{t}}\right)\right) \\
& \sim \downarrow \quad \sim \downarrow \\
& \left(\widetilde{\mathcal{D}}_{\mathfrak{X}^{\prime}} \otimes \widetilde{\omega}_{\mathfrak{X}^{\prime}}^{-1}\right)_{\mathrm{t}} \otimes \widetilde{\mathcal{O}}_{\mathfrak{X}^{\prime}} f_{T}^{!}(\mathcal{E})^{\vee} \longrightarrow \mathbb{R} \mathcal{H o m}_{\widetilde{\mathcal{O}}_{\mathfrak{X}}}\left(f_{T}^{!}(\mathcal{E}),\left(\widetilde{\mathcal{D}}_{\mathfrak{X}^{\prime}} \otimes \widetilde{\omega}_{\mathfrak{X}^{\prime}}^{-1}\right)_{\mathrm{t}}\right) \text {. }
\end{aligned}
$$

3.3.8. - On rappelle que $E$ est un isocristal sur $Y$ surconvergent le long de $T$. On notera par la suite $\mathcal{E}:=\operatorname{sp}_{*}(E), f^{*}(E)$ l'image inverse de $E$ par $f$ (voir $[3,2.3 .2 .2])$ et $f^{*}(\mathcal{E}):=\mathcal{O}_{\mathfrak{X}^{\prime}}\left({ }^{\dagger} T^{\prime}\right)_{\mathbb{Q}} \otimes_{\left.f^{-1} \mathcal{O}_{\mathfrak{X}}{ }^{\dagger} T\right)_{\mathbb{Q}}} f^{-1} \mathcal{E}$. On vérifie à la main la commutativité du diagramme ci-dessous :

$$
\begin{gathered}
\operatorname{sp}_{*} f^{*}\left(E^{\vee}\right) \longrightarrow f^{*} \operatorname{sp}_{*}\left(E^{\vee}\right) \longrightarrow f^{*}\left(\operatorname{sp}_{*} E\right)^{\vee}=f^{*}\left(\mathcal{E}^{\vee}\right) \\
\sim \downarrow \\
\sim \downarrow \\
\operatorname{sp}_{*}\left(f^{*} E\right)^{\vee} \longrightarrow\left(\operatorname{sp}_{*} f^{*} E\right)^{\vee} \longrightarrow\left(f^{*} \operatorname{sp}_{*} E\right)^{\vee}=\left(f^{*} \mathcal{E}\right)^{\vee} .
\end{gathered}
$$

Avec 3.1.3, on a les isomorphismes canoniques $f^{*}(\mathcal{E}) \stackrel{\sim}{\longrightarrow} f_{T}^{!}(\mathcal{E}) \stackrel{\sim}{\longrightarrow} f_{T}^{! \dagger}(\mathcal{E})$. Il résulte alors de l'isomorphisme canonique $f^{*}\left(\mathcal{E}^{\vee}\right) \stackrel{\sim}{\longrightarrow} f^{*}(\mathcal{E})^{\vee}$ les suivants $f_{T}^{!}\left(\mathcal{E}^{\vee}\right) \stackrel{\sim}{\longrightarrow} f_{T}^{!}(\mathcal{E})^{\vee}$ et $\mu: f_{T}^{!^{\dagger}}\left(\mathcal{E}^{\vee}\right) \stackrel{\sim}{\longrightarrow} f_{T}^{!^{\dagger}}(\mathcal{E})^{\vee}$.

Proposition 3.3.9. - Avec les notations 3.3.8, le diagramme

$$
\begin{aligned}
& f_{T}^{!^{\dagger}}\left(\mathbb{D}_{T}^{\dagger}\left(\mathcal{O}_{\mathfrak{X}}\left({ }^{\dagger} T\right)_{\mathbb{Q}}\right) \otimes_{\mathcal{O}_{\mathfrak{X}}\left({ }^{\dagger} T\right)_{\mathbb{Q}}} \mathcal{E}^{\vee}\right) \longrightarrow f_{T}^{!^{\dagger}} \mathbb{D}_{T}^{\dagger}(\mathcal{E}) \\
& \sim \downarrow \\
& \begin{aligned}
f_{T}^{!^{\dagger}} \mathbb{D}_{T}^{\dagger}\left(\mathcal{O}_{\mathfrak{X}}\left({ }^{\dagger} T\right)_{\mathbb{Q}}\right) & \otimes_{\mathcal{O}_{\mathfrak{X}^{\prime}}\left({ }^{\dagger} T^{\prime}\right)_{\mathbb{Q}}} f_{T}^{!^{\dagger}}\left(\mathcal{E}^{\vee}\right) \sim \downarrow \xi \\
& \sim \downarrow \xi \otimes \mu
\end{aligned} \\
& \mathbb{D}_{T^{\prime}}^{\dagger}\left(\mathcal{O}_{\mathfrak{X}^{\prime}}\left({ }^{\dagger} T^{\prime}\right)_{\mathbb{Q}}\right) \otimes_{\mathcal{O}_{\mathfrak{X}^{\prime}}\left({ }^{\dagger} T^{\prime}\right)_{\mathbb{Q}}} f_{T}^{!^{\dagger}}(\mathcal{E})^{\vee} \longrightarrow \mathbb{D}_{T^{\prime}}^{\dagger} f_{T}^{!^{\dagger}}(\mathcal{E}),
\end{aligned}
$$

où les isomorphismes horizontaux dérivent de [10, 2.2.1], est commutatif. 
Démonstration. - Le carré

$$
\begin{aligned}
& f_{T}^{!^{\dagger}} \mathbb{D}_{T}^{\dagger}\left(\mathcal{D}_{\mathfrak{X}}^{\dagger}\left({ }^{\dagger} T\right)_{\mathbb{Q}} \otimes_{\mathcal{D}_{\mathfrak{X}}\left({ }^{\dagger} T\right)_{\mathbb{Q}}} \mathcal{E}\right) \longrightarrow \mathcal{D}_{\mathfrak{X}^{\prime}}^{\dagger}\left({ }^{\dagger} T^{\prime}\right)_{\mathbb{Q}} \otimes_{\mathcal{D}_{\mathfrak{X}^{\prime}}\left({ }^{\dagger} T^{\prime}\right)_{\mathbb{Q}}} f_{T}^{!} \mathbb{D}_{T}(\mathcal{E}) \\
& \sim \downarrow \xi \quad \sim \downarrow \xi \\
& \mathbb{D}_{T^{\prime}}^{\dagger} f_{T}^{!^{\dagger}}\left(\mathcal{D}_{\mathfrak{X}}^{\dagger}\left({ }^{\dagger} T\right)_{\mathbb{Q}} \otimes_{\mathcal{D}_{\mathfrak{X}}\left({ }^{\dagger} T\right)_{\mathbb{Q}}} \mathcal{E}\right) \longrightarrow \mathcal{D}_{\mathfrak{X}^{\prime}}^{\dagger}\left({ }^{\dagger} T^{\prime}\right)_{\mathbb{Q}} \otimes_{\left.\mathcal{D}_{\mathfrak{X}^{\prime}}{ }^{\dagger} T^{\prime}\right)_{\mathbb{Q}}} \mathbb{D}_{T^{\prime}} f_{T}^{!}(\mathcal{E}),
\end{aligned}
$$

où l'isomorphisme de droite se construit de manière analogue à 3.2.7, est commutatif. On se ramène ainsi à prouver la commutativité de 3.3.9.1 sans $\dagger$. Rappelons que les isomorphismes horizontaux de 3.3.9.1 sans $†$ sont construits dans [10, 2.2.1].

D'après 3.3.2, on bénéficie de la commutativité du deuxième carré du haut du diagramme :

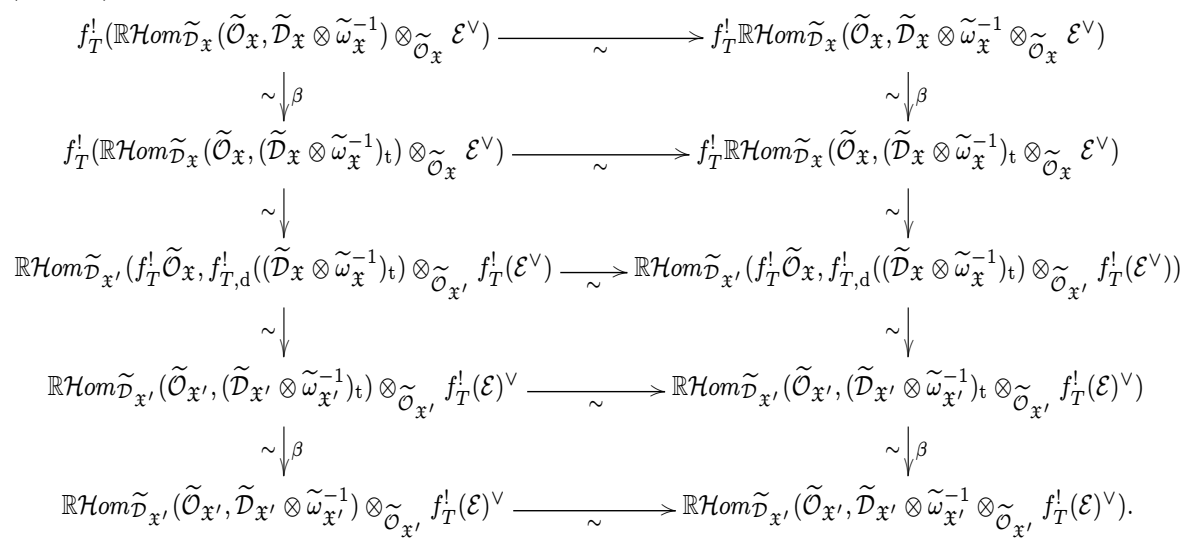

Celle des autres carrés se vérifient par fonctorialité (pour le deuxième du bas, on dispose des isomorphismes : $f_{T, \mathrm{~d}}^{!}\left(\left(\widetilde{\mathcal{D}}_{\mathfrak{X}} \otimes \widetilde{\omega}_{\mathfrak{X}}^{-1}\right)_{\mathrm{t}}\right) \stackrel{\sim}{\longrightarrow}\left(\widetilde{\mathcal{D}}_{\mathfrak{X}^{\prime}} \otimes \widetilde{\omega}_{\mathfrak{X}^{\prime}}^{-1}\right)_{\mathrm{t}}$ et $f_{T}^{!}\left(\mathcal{E}^{\vee}\right) \stackrel{\sim}{\longrightarrow}$ $\left.f_{T}^{!}(\mathcal{E})^{\vee}\right)$. Ainsi, 3.3.9.2 est commutatif.

D'après 3.3.4, le carré de gauche du diagramme :

$$
\begin{aligned}
& \widetilde{\mathcal{D}}_{\mathfrak{X}} \otimes \widetilde{\omega}_{\mathfrak{X}}^{-1} \otimes_{\widetilde{\mathcal{O}}_{\mathfrak{X}}} \mathcal{E}^{\vee} \stackrel{\gamma \otimes \omega^{-1}}{\sim} \mathcal{E}^{\vee} \otimes_{\widetilde{\mathcal{O}}_{\mathfrak{X}}} \widetilde{\mathcal{D}}_{\mathfrak{X}} \otimes \widetilde{\omega}_{\mathfrak{X}}^{-1} \stackrel{\sim}{\sim} \mathbb{R} \mathcal{H}_{\sim^{\prime}} \widetilde{\mathcal{O}}_{\mathfrak{X}}\left(\mathcal{E}, \widetilde{\mathcal{D}}_{\mathfrak{X}} \otimes \widetilde{\omega}_{\mathfrak{X}}^{-1}\right) \\
& \sim \downarrow \beta \quad \sim \downarrow \beta \quad \sim \downarrow \beta
\end{aligned}
$$

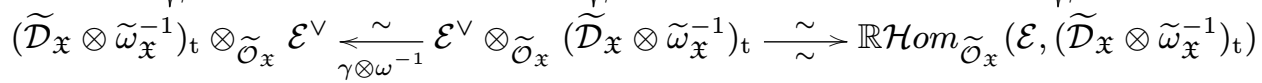

est commutatif. Comme le carré de droite de 3.3.9.3 est commutatif par fonctorialité, il en résulte la commutativité de 3.3.9.3. On dispose du diagramme analogue à 3.3.9.3 en remplaçant $\mathcal{E}$ par $f_{T}^{!}(\mathcal{E})$ et $\mathfrak{X}$ et $\mathfrak{X}^{\prime}$. On construit fonctoriellement à partir de 3.3.9.3 (resp. de son analogue), le carré du haut (resp. 
du bas) du diagramme :

$(3.3 .9 .4)$

$$
\begin{aligned}
& f_{T}^{!} \mathbb{R} \mathcal{H} o m \widetilde{\mathcal{D}}_{\mathfrak{X}}\left(\widetilde{\mathcal{O}}_{\mathfrak{X}}, \widetilde{\mathcal{D}}_{\mathfrak{X}} \otimes \widetilde{\omega}_{\mathfrak{X}}^{-1} \otimes \widetilde{\mathcal{O}}_{\mathfrak{X}} \mathcal{E}^{\vee}\right) \longrightarrow f_{T}^{!} \mathbb{R} \mathcal{H} o \widetilde{\mathcal{D}}_{\mathfrak{X}}\left(\widetilde{\mathcal{O}}_{\mathfrak{X}}, \mathbb{R} \mathcal{H} o m_{\widetilde{\mathcal{O}}_{\mathfrak{X}}}\left(\mathcal{E}, \widetilde{\mathcal{D}}_{\mathfrak{X}} \otimes \widetilde{\omega}_{\mathfrak{X}}^{-1}\right)\right) \\
& \sim \beta_{\beta} \quad \sim{ }_{\beta} \\
& f_{T}^{!} \mathbb{R} \mathcal{H} o m_{\mathcal{D}_{\mathfrak{X}}}\left(\widetilde{\mathcal{O}}_{\mathfrak{X}},\left(\widetilde{\mathcal{D}}_{\mathfrak{X}} \otimes \widetilde{\omega}_{\mathfrak{X}}^{-1}\right)_{\mathrm{t}} \otimes_{\widetilde{\mathcal{O}}_{\mathfrak{X}}} \mathcal{E}^{\vee}\right) \longrightarrow f_{T}^{!} \mathbb{R} \mathcal{H} o m_{\mathcal{D}_{\mathfrak{X}}}\left(\widetilde{\mathcal{O}}_{\mathfrak{X}}, \mathbb{R} \mathcal{H} o m_{\widetilde{\mathcal{O}}_{\mathfrak{X}}}\left(\mathcal{E},\left(\widetilde{\mathcal{D}}_{\mathfrak{X}} \otimes \widetilde{\omega}_{\mathfrak{X}}^{-1}\right)_{\mathrm{t}}\right)\right) \\
& \sim \downarrow \sim \downarrow \\
& \mathbb{R} \mathcal{H} o \widetilde{\mathcal{D}}_{\mathfrak{X}^{\prime}}\left(\widetilde{\mathcal{O}}_{\mathfrak{X}^{\prime}}, f_{T, \mathrm{~d}}^{!}\left(\left(\widetilde{\mathcal{D}}_{\mathfrak{X}} \otimes \widetilde{\omega}_{\mathfrak{X}}^{-1}\right)_{\mathrm{t}} \otimes_{\widetilde{\mathcal{O}}_{\mathfrak{X}}} \mathcal{E}^{\vee}\right)\right) \underset{\sim}{\sim} \mathbb{R} \mathcal{H}{ }^{\prime} \widetilde{\mathcal{D}}_{\mathfrak{X}^{\prime}}\left(\widetilde{\mathcal{O}}_{\mathfrak{X}^{\prime}}, f_{T, \mathrm{~d}}^{!}\left(\mathbb{R} \mathcal{H} o m_{\widetilde{\mathcal{O}}_{\mathfrak{X}}}\left(\mathcal{E},\left(\widetilde{\mathcal{D}}_{\mathfrak{X}} \otimes \widetilde{\omega}_{\mathfrak{X}}^{-1}\right)_{\mathrm{t}}\right)\right)\right)
\end{aligned}
$$

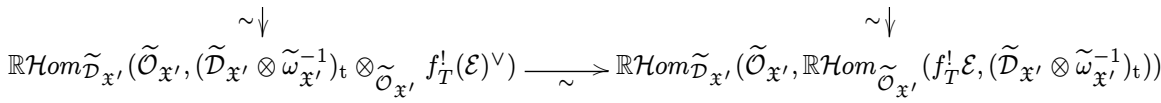

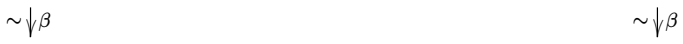

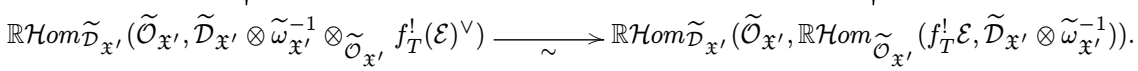

On déduit de 3.3.7.3 que le deuxième carré du bas de 3.3.9.4 est commutatif. Comme les deux autres carrés le sont par fonctorialité, il s'en suit la commutativité de 3.3.9.4.

Comme $f_{T, \mathrm{~d}}^{!}\left(\left(\widetilde{\mathcal{D}}_{\mathfrak{X}} \otimes \widetilde{\omega}_{\mathfrak{X}}^{-1}\right)_{\mathrm{t}}\right) \stackrel{\sim}{\longrightarrow}\left(\widetilde{\mathcal{D}}_{\mathfrak{X}^{\prime}} \otimes \widetilde{\omega}_{\mathfrak{X}^{\prime}}^{-1}\right)_{\mathrm{t}}$ et $f_{T}^{!}\left(\widetilde{\mathcal{O}}_{\mathfrak{X}}\right) \stackrel{\sim}{\longrightarrow} \widetilde{\mathcal{O}}_{\mathfrak{X}^{\prime}}$, il dérive de 3.3.3 le carré du milieu de

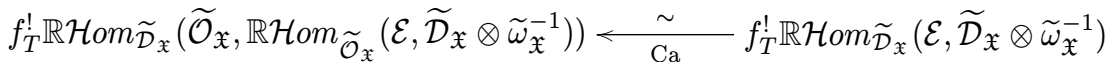

$$
\begin{aligned}
& \sim \downarrow \beta \quad \sim \downarrow \beta
\end{aligned}
$$

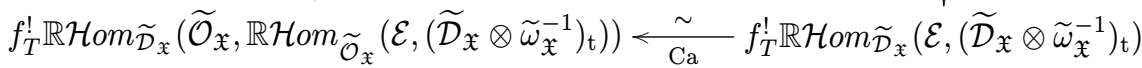

$$
\begin{aligned}
& \sim \downarrow \quad \sim \downarrow \\
& \mathbb{R} \mathcal{H o m}_{\widetilde{\mathcal{D}}_{\mathfrak{X}^{\prime}}}\left(\widetilde{\mathcal{O}}_{\mathfrak{X}^{\prime}}, \mathbb{R} \mathcal{H}_{o m_{\widetilde{\mathcal{O}}_{\mathfrak{X}^{\prime}}}}\left(f_{T}^{!} \mathcal{E},\left(\widetilde{\mathcal{D}}_{\mathfrak{X}^{\prime}} \otimes \widetilde{\omega}_{\mathfrak{X}^{\prime}}^{-1}\right)_{\mathrm{t}}\right)\right) \stackrel{\sim}{\stackrel{\sim}{\mathrm{Ca}}} \mathbb{R} \mathcal{H} o m_{\widetilde{\mathcal{D}}_{\mathfrak{X}^{\prime}}}\left(f_{T}^{!} \mathcal{E},\left(\widetilde{\mathcal{D}}_{\mathfrak{X}^{\prime}} \otimes \widetilde{\omega}_{\mathfrak{X}^{\prime}}^{-1}\right)_{\mathrm{t}}\right) \\
& \sim \downarrow \beta \quad \sim \downarrow \beta
\end{aligned}
$$

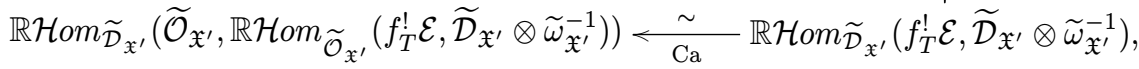

où les flèches horizontales sont les isomorphismes de Cartan ([10, 2.1.34]). Comme les carrés du haut et $\mathrm{du}$ bas sont commutatifs par fonctorialité, le diagramme 3.3.9.5 l'est donc.

On constate que les isomorphismes du haut et du bas de 3.3.9.2 (resp. 3.3.9.4, resp. 3.3.9.5) correspondent à [10, 2.2.1.5] (resp. aux compositions de $[10,2.2 .1 .2]$ avec [10, 2.2.1.4], resp. à [10, 2.2.1.3]). On aboutit alors, en composant $3.3 .9 .2,3.3 .9 .4$ et 3.3 .9 .5 et en ajoutant le décalage $\left[d_{X}\right]$, au diagramme 3.3.9.1 sans $\dagger$. D'où le résultat.

TOME $137-2009-\mathrm{N}^{\circ} 4$ 
3.3.10. - Soient $\mathcal{E} \in D\left(\mathcal{O}_{\mathfrak{X}, \mathbb{Q}}\right)$ et $\mathcal{F} \in D^{+}\left({ }^{\mathrm{g}} \mathcal{D}_{\mathfrak{X}, \mathbb{Q}}^{\dagger},{ }^{\mathrm{g}} \mathcal{D}_{\mathfrak{X}, \mathbb{Q}}^{\dagger}\right)$. De manière analogue à 3.2.6, on dispose des isomorphismes :

$f_{T}^{!}\left(\mathbb{R} \mathcal{H o m}_{\mathcal{O}_{\mathfrak{X}, \mathbb{Q}}}(\mathcal{E}, \mathcal{F})\right) \stackrel{\sim}{\longrightarrow} \mathbb{R} \mathcal{H o m}_{f^{-1} \mathcal{O}_{\mathfrak{X}, \mathbb{Q}}}\left(f^{-1} \mathcal{E}, f_{T, \mathrm{~d}}^{!}(\mathcal{F})\right) \stackrel{\sim}{\longrightarrow} \mathbb{R} \mathcal{H}_{\text {om }}{\mathcal{O}_{\mathfrak{X}^{\prime}, \mathbb{Q}}}\left(f^{*} \mathcal{E}, f_{T, \mathrm{~d}}^{!}(\mathcal{F})\right)$

$$
f^{!}\left(\mathcal{D}_{\mathfrak{X}, \mathbb{Q}}^{\dagger} \otimes \mathcal{O}_{\mathfrak{X}, \mathbb{Q}} \mathcal{E}\right) \stackrel{\sim}{\longrightarrow} f_{\mathrm{g}}^{!}\left(\mathcal{D}_{\mathfrak{X}, \mathbb{Q}}^{\dagger}\right) \otimes_{f^{-1} \mathcal{O}_{\mathfrak{X}, \mathbb{Q}}} f^{-1} \mathcal{E} \stackrel{\sim}{\longrightarrow} \mathcal{D}_{\mathfrak{X}^{\prime}, \mathbb{Q}}^{\dagger} \otimes_{\mathcal{O}_{\mathfrak{X}^{\prime}, \mathbb{Q}}} f^{*}(\mathcal{E}) .
$$

De même, pour tous $\mathcal{E} \in D^{-}\left(\mathcal{O}_{\mathfrak{X}, \mathbb{Q}}\right), \mathcal{F} \in D^{-}\left({ }^{\mathrm{g}} \mathcal{D}_{\mathfrak{X}, \mathbb{Q}}^{\dagger}\left({ }^{\dagger} T\right),{ }^{\mathrm{g}} \mathcal{D}_{\mathfrak{X}, \mathbb{Q}}^{\dagger}\left({ }^{\dagger} T\right)\right)$,

$$
f_{T}^{!}\left(\mathcal{E} \otimes_{\mathcal{O}_{\mathfrak{X}, \mathbb{Q}}}^{\mathbb{L}} \mathcal{F}\right) \stackrel{\sim}{\longrightarrow} f^{-1} \mathcal{E} \otimes_{f^{-1} \mathcal{O}_{\mathfrak{X}, \mathbb{Q}}}^{\mathbb{L}} f_{T, \mathrm{~d}}^{!}(\mathcal{F}) \stackrel{\sim}{\longrightarrow} f^{*} \mathcal{E} \otimes_{\mathcal{O}_{\mathfrak{X}^{\prime}, \mathbb{Q}}}^{\mathbb{L}} f_{T, \mathrm{~d}}^{!}(\mathcal{F})
$$

On dispose d'un isomorphisme canonique $\mathbb{D}_{T}\left(\mathcal{O}_{\mathfrak{X}}\left({ }^{\dagger} T\right)_{\mathbb{Q}}\right) \stackrel{\sim}{\longrightarrow} \mathcal{O}_{\mathfrak{X}}\left({ }^{\dagger} T\right)_{\mathbb{Q}}$. La proposition qui suit signifie que celui-ci commute au foncteur $f_{T}^{!}$. Nous aurons besoin pour sa preuve du lemme ci-dessous.

LEMme 3.3.11. - Soient $\mathcal{E} \in D\left(\mathcal{O}_{\mathfrak{X}, \mathbb{Q}}\right)$ et $\mathcal{F} \in D^{\mathrm{b}}\left({ }^{\mathrm{g}} \mathcal{D}_{\mathfrak{X}, \mathbb{Q}}^{\dagger},{ }^{\mathrm{g}} \mathcal{D}_{\mathfrak{X}, \mathbb{Q}}^{\dagger}\right)$. Le diagramme canonique

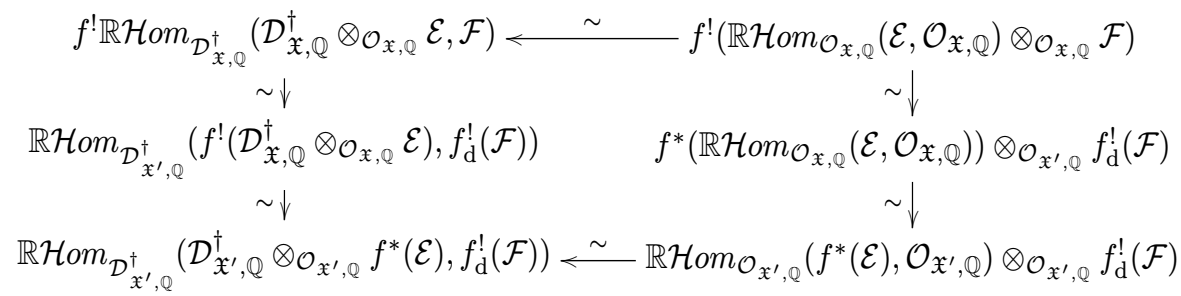

est commutatif.

Démonstration. - Pour construire trois des flèches verticales de 3.3.11.1, on a utilisé respectivement 3.3.10.2, 3.3.10.1 et 3.2.6.1. Le diagramme 3.3.11.1 est le composé des deux diagrammes suivants :

$$
\begin{aligned}
& f^{!} \mathbb{R} \mathcal{H o m}_{\mathcal{D}_{\mathfrak{X}, \mathbb{Q}}^{\dagger}}\left(\mathcal{D}_{\mathfrak{X}, \mathbb{Q}}^{\dagger} \otimes_{\mathcal{O}_{\mathfrak{X}, \mathbb{Q}}} \mathcal{E}, \mathcal{F}\right) \longleftarrow f^{!} \mathbb{R} \mathcal{H}^{\circ} m_{\mathcal{O}_{\mathfrak{X}, \mathbb{Q}}}(\mathcal{E}, \mathcal{F})
\end{aligned}
$$

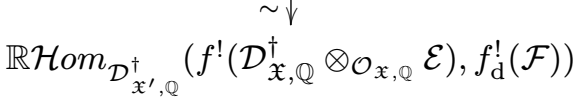

$$
\begin{aligned}
& \sim \downarrow \\
& \mathbb{R} \mathcal{H} o m_{\mathcal{D}_{\mathfrak{X}^{\prime}, \mathbb{Q}}^{\dagger}}\left(\mathcal{D}_{\mathfrak{X}^{\prime}, \mathbb{Q}}^{\dagger} \otimes_{\mathcal{O}_{\mathfrak{X}^{\prime}, \mathbb{Q}}} f^{*}(\mathcal{E}), f_{\mathrm{d}}^{!}(\mathcal{F})\right) \longleftarrow \sim \sim \mathcal{R} \mathcal{H o m}_{\mathcal{O}_{\mathfrak{X}^{\prime}, \mathbb{Q}}}\left(f^{*}(\mathcal{E}), f_{\mathrm{d}}^{!}(\mathcal{F})\right),
\end{aligned}
$$


$(3.3 .11 .3)$

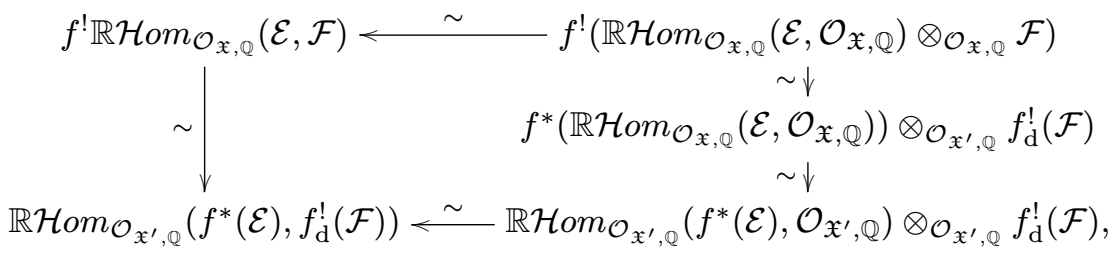

dont la commutativité se vérifie, après avoir résolu injectivement $\mathcal{F}$, par un calcul.

Proposition 3.3.12. - Le diagramme canonique suivant

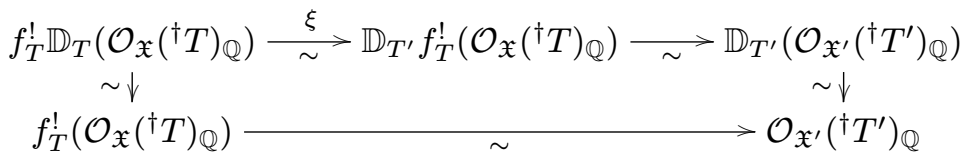

est commutatif.

Démonstration. - Par [4, 4.3.12], il suffit de prouver la proposition lorsque $T$ est vide. On notera $\widetilde{\mathcal{D}}_{\mathfrak{X}, \mathbb{Q}}^{\dagger}:=\mathcal{D}_{\mathfrak{X}, \mathbb{Q}}^{\dagger} \otimes_{\mathcal{O}_{\mathfrak{X}}} \omega_{\mathfrak{X}}^{-1}$ et $\left(\widetilde{\mathcal{D}}_{\mathfrak{X}, \mathbb{Q}}^{\dagger}\right)_{\mathrm{t}}:=\left(\mathcal{D}_{\mathfrak{X}, \mathbb{Q}}^{\dagger} \otimes_{\mathcal{O}_{\mathfrak{X}}} \omega_{\mathfrak{X}}^{-1}\right)_{\mathrm{t}}$. Notons $\mathcal{T}_{\mathfrak{X}}$ le faisceau tangent de $\mathfrak{X}$ et $\mathcal{D}_{\mathfrak{X}, \mathbb{Q}}^{\dagger} \otimes_{\mathcal{O}_{\mathfrak{X}}} \wedge^{\bullet} \mathcal{T}_{\mathfrak{X}}$ le complexe de Spencer

$$
\mathcal{D}_{\mathfrak{X}, \mathbb{Q}}^{\dagger} \otimes_{\mathcal{O}_{\mathfrak{X}}} \wedge^{\mathrm{d}} \mathcal{T}_{\mathfrak{X}} \rightarrow \cdots \rightarrow \mathcal{D}_{\mathfrak{X}, \mathbb{Q}}^{\dagger} \otimes_{\mathcal{O}_{\mathfrak{X}}} \mathcal{T}_{\mathfrak{X}} \rightarrow \mathcal{D}_{\mathfrak{X}, \mathbb{Q}}^{\dagger}
$$

(voir [5, 4.3.1]). L'application canonique $\mathcal{D}_{\mathfrak{X}, \mathbb{Q}}^{\dagger} \rightarrow \mathcal{O}_{\mathfrak{X}, \mathbb{Q}}$ envoyant un opérateur $P$ sur $P \cdot 1$ induit un quasi-isomorphisme $\mathcal{D}_{\mathfrak{X}, \mathbb{Q}}^{\dagger} \otimes_{\mathcal{O}_{\mathfrak{X}}} \wedge \mathcal{T}_{\mathfrak{X}} \stackrel{\sim}{\longrightarrow} \mathcal{O}_{\mathfrak{X}, \mathbb{Q}}$. L'isomorphisme canonique $f^{*} \Omega_{\mathfrak{X}} \stackrel{\sim}{\longrightarrow} \Omega_{\mathfrak{X}^{\prime}}$ induit par dualité le suivant $\mathcal{T}_{\mathfrak{X}^{\prime}} \stackrel{\sim}{\longrightarrow} f^{*} \mathcal{T}_{\mathfrak{X}}$. Avec 3.3.10.1, on en déduit, pour tout entier $r$, le composé : $f^{!}\left(\mathcal{D}_{\mathfrak{X}, \mathbb{Q}}^{\dagger} \otimes_{\mathcal{O}_{\mathfrak{X}}}\right.$ $\left.\wedge^{r} \mathcal{T}_{\mathfrak{X}}\right) \stackrel{\sim}{\longrightarrow} \mathcal{D}_{\mathfrak{X}^{\prime}, \mathbb{Q}}^{\dagger} \otimes_{\mathcal{O}_{\mathfrak{X}^{\prime}}} f^{*}\left(\wedge^{r} \mathcal{T}_{\mathfrak{X}}\right) \stackrel{\sim}{\longleftarrow} \mathcal{D}_{\mathfrak{X}^{\prime}, \mathbb{Q}}^{\dagger} \otimes_{\mathcal{O}_{\mathfrak{X}^{\prime}}} \wedge^{r} \mathcal{T}_{\mathfrak{X}^{\prime}}$. On construit ainsi le diagramme suivant :

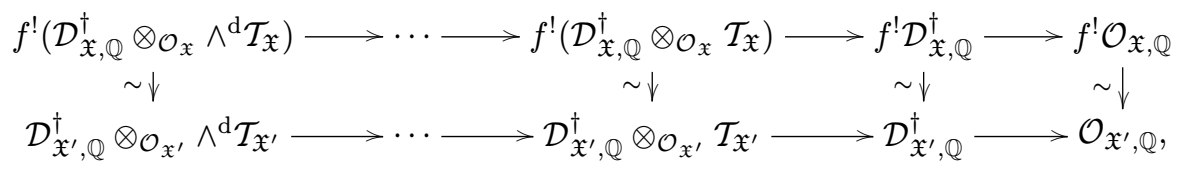

dont les complexes horizontaux sont exacts ( $f^{!}$est exact). On vérifie de plus que 3.3.12.2 est commutatif. On obtient en particulier un isomorphisme $f^{!}\left(\mathcal{D}_{\mathfrak{X}, \mathbb{Q}}^{\dagger} \otimes_{\mathcal{O}_{\mathfrak{X}}} \wedge^{\bullet} \mathcal{T}_{\mathfrak{X}}\right) \stackrel{\sim}{\longrightarrow} \mathcal{D}_{\mathfrak{X}^{\prime}, \mathbb{Q}}^{\dagger} \otimes_{\mathcal{O}_{\mathfrak{X}^{\prime}}} \wedge^{\bullet} \mathcal{T}_{\mathfrak{X}^{\prime}}$. Il en dérive aussi par dualité la 
commutativité du carré du bas de :

$(3.3 .12 .3)$

$$
\begin{aligned}
& f^{!} \mathbb{R} \mathcal{H} o m_{\mathcal{D}_{\mathfrak{X}, \mathbb{Q}}^{\dagger}}\left(\mathcal{O}_{\mathfrak{X}, \mathbb{Q}}, \widetilde{\mathcal{D}}_{\mathfrak{X}, \mathbb{Q}}^{\dagger}\right) \longrightarrow f^{!} \mathcal{H}^{\circ} m_{\mathcal{D}_{\mathfrak{X}, \mathbb{Q}}^{\dagger}}\left(\mathcal{D}_{\mathfrak{X}, \mathbb{Q}}^{\dagger} \otimes_{\mathcal{O}_{\mathfrak{X}}} \wedge \cdot \mathcal{T}_{\mathfrak{X}}, \widetilde{\mathcal{D}}_{\mathfrak{X}, \mathbb{Q}}^{\dagger}\right) \\
& \sim \downarrow \xi \quad \sim \downarrow \xi \\
& \mathbb{R} \mathcal{H}_{o m_{\mathcal{D}_{\mathfrak{X}^{\prime}, \mathbb{Q}}^{\dagger}}^{\dagger}}\left(f^{!} \mathcal{O}_{\mathfrak{X}, \mathbb{Q}}, \widetilde{\mathcal{D}}_{\mathfrak{X}^{\prime}, \mathbb{Q}}^{\dagger}\right) \underset{\sim}{\longrightarrow} \mathcal{H}^{\prime} m_{\mathcal{D}_{\mathfrak{X}^{\prime}, \mathbb{Q}}^{\dagger}}\left(f^{!}\left(\mathcal{D}_{\mathfrak{X}, \mathbb{Q}}^{\dagger} \otimes_{\mathcal{O}_{\mathfrak{X}}} \wedge \cdot \mathcal{T}_{\mathfrak{X}}\right), \widetilde{\mathcal{D}}_{\mathfrak{X}^{\prime}, \mathbb{Q}}^{\dagger}\right)
\end{aligned}
$$

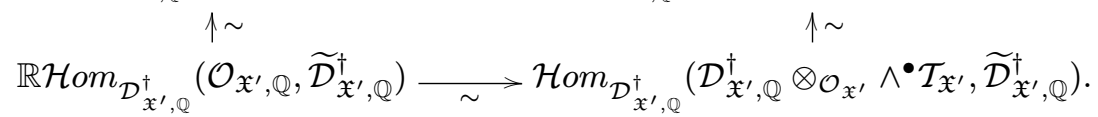

On établit par fonctorialité la commutativité du carré du haut de 3.3.12.3. D'où celle de 3.3.12.3.

Le diagramme

$$
\begin{aligned}
& f^{!} \mathcal{H}^{\prime} m_{\mathcal{D}_{\mathfrak{X}, \mathbb{Q}}^{\dagger}}\left(\mathcal{D}_{\mathfrak{X}, \mathbb{Q}}^{\dagger} \otimes \mathcal{O}_{\mathfrak{X}, \mathbb{Q}} \wedge \mathcal{T}_{\mathfrak{X}, \mathbb{Q}}, \widetilde{\mathcal{D}}_{\mathfrak{X}, \mathbb{Q}}^{\dagger}\right) \longrightarrow f^{!}\left(\mathcal{H}^{\prime} m_{\mathcal{O}_{\mathfrak{X}, \mathbb{Q}}}\left(\wedge \mathcal{T}_{\mathfrak{X}, \mathbb{Q}}, \mathcal{O}_{\mathfrak{X}, \mathbb{Q}}\right) \otimes \mathcal{O}_{\mathfrak{X}, \mathbb{Q}} \widetilde{\mathcal{D}}_{\mathfrak{X}, \mathbb{Q}}^{\dagger}\right) \\
& \sim \gamma^{\prime} \quad \sim \gamma^{\beta} \\
& f^{!} \mathcal{H}^{\prime} m_{\mathcal{D}_{\mathfrak{X}, \mathbb{Q}}^{\dagger}}\left(\mathcal{D}_{\mathfrak{X}, \mathbb{Q}}^{\dagger} \otimes_{\mathcal{O}_{\mathfrak{X}, \mathbb{Q}}} \wedge^{\bullet} \mathcal{T}_{\mathfrak{X}, \mathbb{Q}},\left(\widetilde{\mathcal{D}}_{\mathfrak{X}, \mathbb{Q}}^{\dagger}\right) \mathrm{t}\right) \longrightarrow f^{!}\left(\mathcal{H}^{\prime} m_{\mathcal{O}_{\mathfrak{X}, \mathbb{Q}}}\left(\wedge^{\bullet} \mathcal{T}_{\mathfrak{X}, \mathbb{Q}}, \mathcal{O}_{\mathfrak{X}, \mathbb{Q}}\right) \otimes_{\mathcal{O}_{\mathfrak{X}, \mathbb{Q}}}\left(\widetilde{\mathcal{D}}_{\mathfrak{X}, \mathbb{Q}}^{\dagger}\right)_{\mathrm{t}}\right) \\
& \mathcal{H o m}_{\mathcal{D}_{\mathfrak{X}^{\prime}, \mathbb{Q}}^{\dagger}}\left(\mathcal{D}_{\mathfrak{X}^{\prime}, \mathbb{Q}}^{\dagger} \otimes_{\mathcal{O}_{\mathfrak{X}^{\prime}, \mathbb{Q}}} f^{*}\left(\wedge \bullet \mathcal{T}_{\mathfrak{X}, \mathbb{Q}}\right), f_{\mathrm{d}}^{!}\left(\left(\widetilde{\mathcal{D}}_{\mathfrak{X}, \mathbb{Q}}^{\dagger}\right) t\right)\right) \underset{\sim}{\sim} \mathcal{H o m}_{\mathcal{O}_{\mathfrak{X}^{\prime}, \mathbb{Q}}}\left(f^{*}\left(\wedge \bullet \mathcal{T}_{\mathfrak{X}, \mathbb{Q}}\right), \mathcal{O}_{\mathfrak{X}^{\prime}, \mathbb{Q}}\right) \otimes_{\mathcal{O}_{\mathfrak{X}^{\prime}, \mathbb{Q}}} f_{\mathrm{d}}^{!}\left(\left(\widetilde{\mathcal{D}}_{\mathfrak{X}, \mathbb{Q}}^{\dagger}\right) t\right) \\
& \mathcal{H}_{\mathcal{D}_{\mathfrak{X}^{\prime}, \mathbb{Q}}^{\dagger}}\left(\mathcal{D}_{\mathfrak{X}^{\prime}, \mathbb{Q}}^{\dagger} \otimes \mathcal{O}_{\mathfrak{X}^{\prime}, \mathbb{Q}} \wedge \wedge^{\bullet} \mathcal{T}_{\mathfrak{X}^{\prime}, \mathbb{Q}},\left(\widetilde{\mathcal{D}}_{\mathfrak{X}^{\prime}, \mathbb{Q}}^{\dagger}\right)_{\mathrm{t}}\right) \longrightarrow \sim \mathcal{H}_{\sim} \underset{\mathcal{O}_{\mathfrak{X}^{\prime}, \mathbb{Q}}}{ }\left(\wedge^{\bullet} \mathcal{T}_{\left.\mathfrak{X}^{\prime}, \mathcal{O}_{\mathfrak{X}^{\prime}, \mathbb{Q}}\right) \otimes \mathcal{O}_{\mathfrak{X}^{\prime}, \mathbb{Q}}}\left(\widetilde{\mathcal{D}}_{\mathfrak{X}^{\prime}, \mathbb{Q}}^{\dagger}\right)_{\mathrm{t}}\right. \\
& \sim \gamma_{\beta} \quad \sim \gamma^{\beta}
\end{aligned}
$$

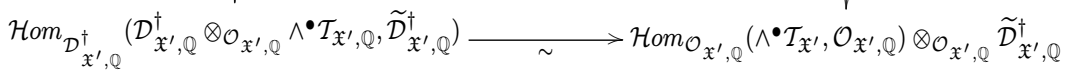

est commutatif. En effet, pour le carré du haut et les deux du bas, cela se vérifie par fonctorialité tandis que le dernier résulte de 3.3.11.1.

Les deux carrés du haut et celui du bas du diagramme

$$
\begin{aligned}
& f^{!}\left(\mathcal{H}^{\circ o m_{\mathcal{O}_{\mathfrak{Q}}}}\left(\wedge^{\bullet} \mathcal{T}_{\mathfrak{X}, \mathbb{Q}}, \mathcal{O}_{\mathfrak{X}, \mathbb{Q}}\right) \otimes_{\mathcal{O}_{\mathfrak{X}, \mathbb{Q}}} \widetilde{\mathcal{D}}_{\mathfrak{X}, \mathbb{Q}}^{\dagger}\right) \longrightarrow f^{!}\left(\Omega_{\mathfrak{X}, \mathbb{Q}}^{\bullet} \otimes_{\mathcal{O}_{\mathfrak{X}, \mathbb{Q}}} \widetilde{\mathcal{D}}_{\mathfrak{X}, \mathbb{Q}}^{\dagger}\right) \\
& \sim \downarrow \beta \quad \sim \downarrow \beta \\
& f^{!}\left(\mathcal{H}^{\prime} m_{\mathcal{O}_{\mathfrak{X}, \mathbb{Q}}}\left(\wedge^{\bullet} \mathcal{T}_{\mathfrak{X}, \mathbb{Q}}, \mathcal{O}_{\mathfrak{X}, \mathbb{Q}}\right) \otimes_{\mathcal{O}_{\mathfrak{X}, \mathbb{Q}}}\left(\widetilde{\mathcal{D}}_{\mathfrak{X}, \mathbb{Q}}^{\dagger}\right)_{\mathrm{t}}\right) \longrightarrow f^{!}\left(\Omega_{\mathfrak{X}, \mathbb{Q}}^{\bullet} \otimes_{\mathcal{O}_{\mathfrak{X}, \mathbb{Q}}}\left(\widetilde{\mathcal{D}}_{\mathfrak{X}, \mathbb{Q}}^{\dagger}\right)_{\mathrm{t}}\right) \\
& \sim \downarrow \quad \sim \downarrow \\
& f^{*} \mathcal{H o m}_{\mathcal{O}_{\mathfrak{X}, \mathbb{Q}}}\left(\wedge^{\bullet} \mathcal{T}_{\mathfrak{X}, \mathbb{Q}}, \mathcal{O}_{\mathfrak{X}, \mathbb{Q}}\right) \otimes_{\mathcal{O}_{\mathfrak{X}^{\prime}, \mathbb{Q}}} f_{\mathrm{d}}^{!}\left(\left(\widetilde{\mathcal{D}}_{\mathfrak{X}, \mathbb{Q}}^{\dagger}\right)_{\mathrm{t}}\right) \longrightarrow f^{*}\left(\Omega_{\mathfrak{X}, \mathbb{Q}}^{\bullet}\right) \otimes_{\mathcal{O}_{\mathfrak{X}^{\prime}, \mathbb{Q}}} f_{\mathrm{d}}^{!}\left(\left(\widetilde{\mathcal{D}}_{\mathfrak{X}, \mathbb{Q}}^{\dagger}\right)_{\mathrm{t}}\right) \\
& \sim \downarrow \quad \sim \downarrow \\
& \mathcal{H o m}_{\mathcal{O}_{\mathfrak{X}^{\prime}, \mathbb{Q}}}\left(\wedge^{\bullet} \mathcal{T}_{\mathfrak{X}^{\prime}, \mathbb{Q}}, \mathcal{O}_{\mathfrak{X}^{\prime}, \mathbb{Q}}\right) \otimes_{\mathcal{O}_{\mathfrak{X}^{\prime}, \mathbb{Q}}} f_{\mathrm{d}}^{!}\left(\left(\widetilde{\mathcal{D}}_{\mathfrak{X}, \mathbb{Q}}^{\dagger}\right)_{\mathrm{t}}\right) \longrightarrow \Omega_{\mathfrak{X}^{\prime}, \mathbb{Q}}^{\bullet} \otimes_{\mathcal{O}_{\mathfrak{X}^{\prime}, \mathbb{Q}}} f_{\mathrm{d}}^{!}\left(\left(\widetilde{\mathcal{D}}_{\mathfrak{X}, \mathbb{Q}}^{\dagger}\right)_{\mathrm{t}}\right) \\
& \sim \downarrow \quad \sim \downarrow \\
& \mathcal{H} m_{\mathcal{O}_{\mathfrak{X}^{\prime}, \mathbb{Q}}}\left(\wedge^{\bullet} \mathcal{T}_{\mathfrak{X}^{\prime}, \mathbb{Q}}, \mathcal{O}_{\mathfrak{X}^{\prime}, \mathbb{Q}}\right) \otimes_{\mathcal{O}_{\mathfrak{X}^{\prime}, \mathbb{Q}}} \widetilde{\mathcal{D}}_{\mathfrak{X}^{\prime}, \mathbb{Q}}^{\dagger} \longrightarrow \Omega_{\mathfrak{X}^{\prime}, \mathbb{Q}}^{\bullet} \otimes_{\mathcal{O}_{\mathfrak{X}^{\prime}, \mathbb{Q}}} \widetilde{\mathcal{D}}_{\mathfrak{X}^{\prime}, \mathbb{Q}}^{\dagger},
\end{aligned}
$$


dont les flèches verticales du bas sont induits par $f_{\mathrm{d}}^{!}\left(\left(\widetilde{\mathcal{D}}_{\mathfrak{X}, \mathbb{Q}}^{\dagger}\right)_{\mathrm{t}}\right) \stackrel{\sim}{\longrightarrow}\left(\widetilde{\mathcal{D}}_{\mathfrak{X}^{\prime}, \mathbb{Q}}^{\dagger}\right)_{\mathrm{t}} \underset{\beta}{\stackrel{\sim}{\longrightarrow}}$ $f_{\mathrm{d}}^{!}\left(\left(\widetilde{\mathcal{D}}_{\mathfrak{X}, \mathbb{Q}}^{\dagger}\right)_{\mathrm{t}}\right)$, sont commutatifs par fonctorialité. Le dernier l'est par définition.

Par un calcul local, on établit la commutativité du diagramme ci-après :

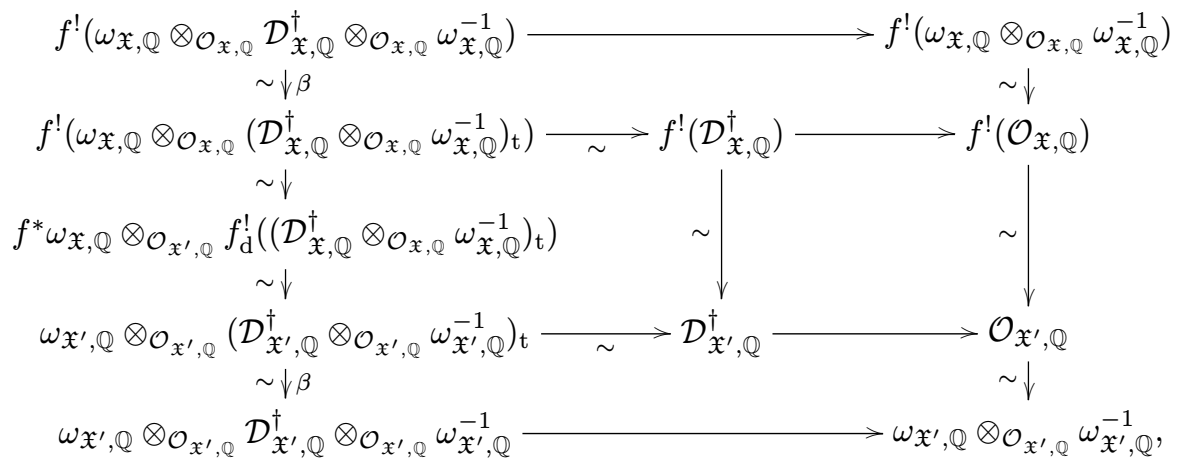

où la flèche horizontale du haut (resp. du bas) est induite par l'action à droite de $\mathcal{D}_{\mathfrak{X}, \mathbb{Q}}^{\dagger}$ sur $\omega_{\mathfrak{X}, \mathbb{Q}}$ (resp. de $\mathcal{D}_{\mathfrak{X}^{\prime}, \mathbb{Q}}^{\dagger}$ sur $\omega_{\mathfrak{X}^{\prime}, \mathbb{Q}}$ ) tandis que celles du carré de droite découlent de l'action à gauche de $\mathcal{D}_{\mathfrak{X}, \mathbb{Q}}^{\dagger}$ sur $\mathcal{O}_{\mathfrak{X}, \mathbb{Q}}$ ou de celle de $\mathcal{D}_{\mathfrak{X}^{\prime}, \mathbb{Q}}^{\dagger}$ $\operatorname{sur} \mathcal{O}_{\mathfrak{X}^{\prime}, \mathbb{Q}}$. On dispose d'un morphisme canonique entre les flèches de droite de 3.3.12.5 décalées de $\left[d_{X}\right]$ et celle de gauche de 3.3.12.6. Via ce morphisme, en composant 3.3.12.3, 3.3.12.4, 3.3.12.5 décalés de $\left[d_{X}\right]$ avec 3.3.12.6, on obtient le diagramme commutatif de 3.3.12.

Corollaire 3.3.13. - Avec les notations 3.3.8, on suppose ici que $f$ est une immersion ouverte. Le diagramme

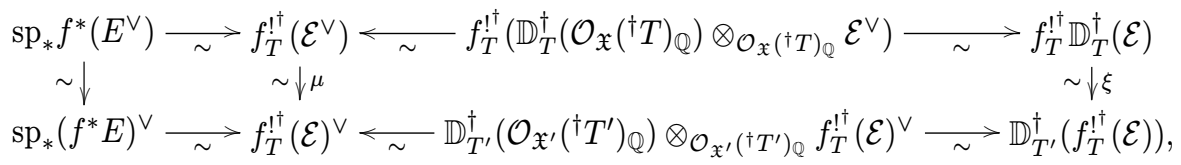

où $\mu$ et $\xi$ ont été défini respectivement dans 3.3.8 et 3.2.7, est commutatif. 
Démonstration. - La commutativité du carré de gauche est tautologique (voir 3.3.8). Il résulte par fonctorialité de 3.3.12 que le rectangle du milieu du diagramme :

$$
\begin{aligned}
& f_{T}^{!^{\dagger}}\left(\mathcal{O}_{\mathfrak{X}}\left({ }^{\dagger} T\right)_{\mathbb{Q}} \otimes_{\mathcal{O}_{\mathfrak{X}}\left({ }^{\dagger} T\right)_{\mathbb{Q}}} \mathcal{E}^{\vee}\right) \longleftarrow f_{T}^{!^{\dagger}}\left(\mathbb{D}_{T}^{\dagger}\left(\mathcal{O}_{\mathfrak{X}}\left({ }^{\dagger} T\right)_{\mathbb{Q}}\right) \otimes_{\mathcal{O}_{\mathfrak{X}}\left({ }^{\dagger} T\right)_{\mathbb{Q}}} \mathcal{E}^{\vee}\right) \\
& \sim \downarrow \quad \sim \downarrow \\
& f_{T}^{!^{\dagger}}\left(\mathcal{O}_{\mathfrak{X}}\left({ }^{\dagger} T\right)_{\mathbb{Q}}\right) \otimes_{\mathcal{O}_{\mathfrak{X}^{\prime}}\left({ }^{\dagger} T^{\prime}\right)_{\mathbb{Q}}} f_{T}^{!^{\dagger}}\left(\mathcal{E}^{\vee}\right)<\sim f_{T}^{!^{\dagger}} \mathbb{D}_{T}^{\dagger}\left(\mathcal{O}_{\mathfrak{X}}\left({ }^{\dagger} T\right)_{\mathbb{Q}}\right) \otimes_{\left.\mathcal{O}_{\mathfrak{X}^{\prime}}{ }^{\dagger} T^{\prime}\right)_{\mathbb{Q}}} f_{T}^{!^{\dagger}}\left(\mathcal{E}^{\vee}\right) \\
& \sim \downarrow \xi \otimes \mathrm{Id} \\
& \sim \downarrow \begin{aligned}
\mathbb{D}_{T}^{\dagger} f_{T}^{!^{\dagger}}\left(\mathcal{O}_{\mathfrak{X}}\left({ }^{\dagger} T\right)_{\mathbb{Q}}\right) & \otimes_{\mathcal{O}_{\mathfrak{X}^{\prime}}\left({ }^{\dagger} T^{\prime}\right)_{\mathbb{Q}}} f_{T}^{!_{T}^{\dagger}}\left(\mathcal{E}^{\vee}\right) \\
\sim \downarrow & \sim
\end{aligned} \\
& \mathcal{O}_{\mathfrak{X}^{\prime}}\left({ }^{\dagger} T^{\prime}\right)_{\mathbb{Q}} \otimes_{\mathcal{O}_{\mathfrak{X}^{\prime}}\left({ }^{\dagger} T^{\prime}\right)_{\mathbb{Q}}} f_{T}^{!^{\dagger}}\left(\mathcal{E}^{\vee}\right) \ll \sim \sim \mathbb{D}_{T}^{\dagger}\left(\mathcal{O}_{\mathfrak{X}^{\prime}}\left({ }^{\dagger} T^{\prime}\right)_{\mathbb{Q}}\right) \otimes_{\mathcal{O}_{\mathfrak{X}^{\prime}}\left({ }^{\dagger} T^{\prime}\right)_{\mathbb{Q}}} f_{T}^{!^{\dagger}}\left(\mathcal{E}^{\vee}\right) \\
& \sim \downarrow \mu \quad \sim \downarrow \mu \\
& \mathcal{O}_{\mathfrak{X}^{\prime}}\left({ }^{\dagger} T^{\prime}\right)_{\mathbb{Q}} \otimes_{\mathcal{O}_{\mathfrak{X}^{\prime}}\left({ }^{\dagger} T^{\prime}\right)_{\mathbb{Q}}} f_{T}^{!^{\dagger}}(\mathcal{E})^{\vee} \prec \sim \mathbb{D}_{T}^{\dagger}\left(\mathcal{O}_{\mathfrak{X}^{\prime}}\left({ }^{\dagger} T^{\prime}\right)_{\mathbb{Q}}\right) \otimes_{\mathcal{O}_{\mathfrak{X}^{\prime}}\left({ }^{\dagger} T^{\prime}\right)_{\mathbb{Q}}} f_{T}^{!^{\dagger}}(\mathcal{E})^{\vee}
\end{aligned}
$$

est commutatif. Comme les carrés supérieur et inférieur le sont par fonctorialité, 3.3.13.2 est commutatif. En composant 3.3.13.2 et 3.3.9.1, on obtient 3.3.13.1.

Remarques 3.3.14. - Avec les notations 3.3.8, on se note $g: \mathfrak{X}^{\prime \prime} \hookrightarrow \mathfrak{X}^{\prime}$ une seconde immersion ouverte et $T^{\prime \prime}:=g_{0}^{-1}\left(T^{\prime}\right)$. On bénéficie par fonctorialité du diagramme commutatif :

(3.3.14.1)

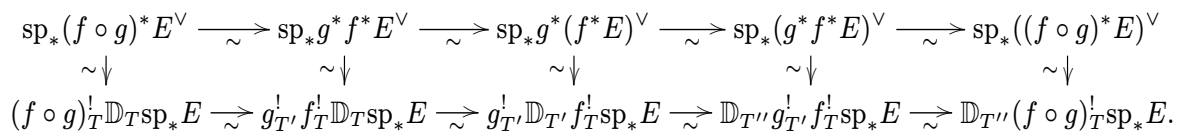

De plus, par transitivité des isomorphismes de commutation des duaux aux images inverses (extraordinaires) le composé du haut est l'isomorphisme canonique $\operatorname{sp}_{*}(f \circ g)^{*} E^{\vee} \stackrel{\sim}{\longrightarrow} \operatorname{sp}_{*}\left((f \circ g)^{*} E\right)^{\vee}$ et celui du bas est $(f \circ g)_{T}^{!} \mathbb{D}_{T} \operatorname{sp}_{*} E \stackrel{\sim}{\longrightarrow}$ $\mathbb{D}_{T^{\prime \prime}}(f \circ g)_{T}^{!} \operatorname{sp}_{*} E$.

\section{Commutation de $\mathrm{sp}_{+}$aux opérations cohomologiques}

\subsection{Commutation aux foncteurs restrictions et images inverses extraordinaires. -}

Nous reprenons les notations de 2.5.1. La proposition qui suit est immédiate.

Proposition 4.1.1. - Soient $E$ un isocristal sur $Y$ surconvergent le long de $T_{X}, \mathcal{P}^{\prime}$ un ouvert de $\mathcal{P}, X^{\prime}:=X \cap P^{\prime}$ et $T^{\prime}:=T \cap P^{\prime}$. Il existe alors un isomorphisme canonique $:\left.\operatorname{sp}_{X \hookrightarrow \mathcal{P}, T+}(E)\right|_{\mathcal{P}^{\prime}} \stackrel{\sim}{\longrightarrow} \operatorname{sp}_{X^{\prime} \hookrightarrow \mathcal{P}^{\prime}, T^{\prime}+}\left(\left.E\right|_{X_{X^{\prime}}\left[\mathcal{P}^{\prime}\right.}\right)$ fonctoriel en E. 
En particulier, en notant $\widehat{E}$ l'isocristal convergeant sur $Y$ associé à E, $\left.\operatorname{sp}_{X \hookrightarrow \mathcal{P}, T+}(E)\right|_{\mathfrak{U}} \stackrel{\sim}{\longrightarrow} \operatorname{sp}_{Y \hookrightarrow \mathfrak{U}+}(\widehat{E})$.

Proposition 4.1.2. - Soient $T^{\prime} \subset T$ un second diviseur de $X$ et $E$ un isocristal sur $X \backslash T^{\prime}$ surconvergent le long de $T^{\prime}$. On dispose de l'isomorphisme : $\left({ }^{\dagger} T\right) \operatorname{sp}_{X \hookrightarrow \mathcal{P}, T^{\prime}+}(E) \stackrel{\sim}{\longrightarrow} \operatorname{sp}_{X \hookrightarrow \mathcal{P}, T+}\left(j^{\dagger} E\right)$ fonctoriel en $E$.

La preuve étant identique dans le cas général et afin de ne pas alourdir les notations, supposons $T^{\prime}=\varnothing$. Avec les arguments du début 2.5.1, on peut aussi supposer $X$ intègre. La proposition résulte des trois lemmes qui suivent.

LEMme 4.1.3. - Si $\left(\left(E_{\alpha}\right)_{\alpha \in \Lambda},\left(\eta_{\alpha \beta}\right)_{\alpha, \beta \in \Lambda}\right) \in \operatorname{Isoc}^{\dagger}\left(X, X,\left(\mathcal{P}_{\alpha}\right)_{\alpha \in \Lambda} / K\right)$ se recolle en $E \in \operatorname{Isoc}^{\dagger}(X, X / K)$, alors $\left(\left(j_{\alpha}^{\dagger} E_{\alpha}\right)_{\alpha \in \Lambda},\left(\eta_{\alpha \beta}^{\prime}\right)_{\alpha, \beta \in \Lambda}\right)$ se recolle en $j^{\dagger} E$, où $\eta_{\alpha \beta}^{\prime}$ est l'unique isomorphisme rendant commutatif le diagramme :

$$
\begin{aligned}
& p_{1 K}^{\alpha \beta *}\left(j_{\alpha}^{\dagger} E_{\alpha}\right) \longrightarrow j_{\alpha \beta}^{\dagger} p_{1 K}^{\alpha \beta *}\left(E_{\alpha}\right) \\
& \eta_{\alpha \beta}^{\prime} 今 \quad j_{\alpha \beta}^{\dagger} \eta_{\alpha \beta} \uparrow \sim \\
& p_{2 K}^{\alpha \beta *}\left(j_{\beta}^{\dagger} E_{\beta}\right) \longrightarrow j_{\alpha \beta}^{\dagger} p_{2 K}^{\alpha \beta *}\left(E_{\beta}\right),
\end{aligned}
$$

dont les isomorphismes horizontaux sont ceux de la commutation à l'image inverse des foncteurs de la forme $j^{\dagger}$.

Démonstration. - i) Dans un premier temps, vérifions que la famille d'isomorphismes $\eta_{\alpha \beta}^{\prime}$ définit bien une donnée de recollement.

Soit le diagramme

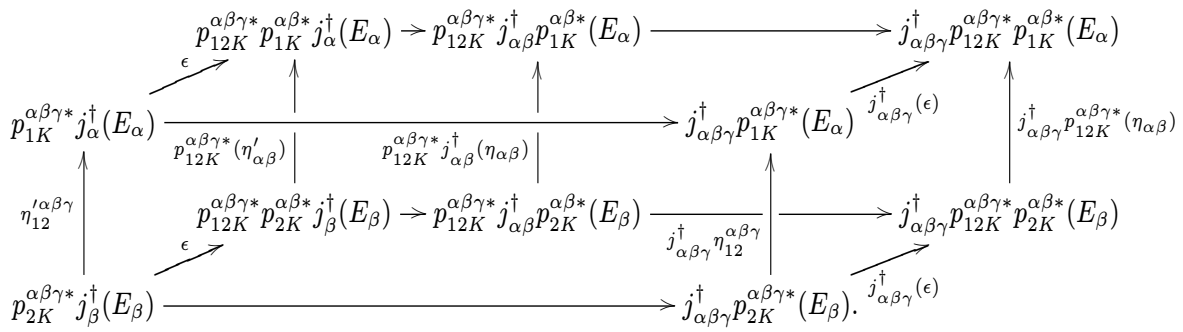

Les carrés du fond, de droite et de gauche sont commutatifs par définition ou par fonctorialité. De plus, grâce à 2.3.1.2 et à la transitivité de l'isomorphisme de commutation des foncteurs de la forme $j^{\dagger}$ aux images inverses, il en est de même des diagrammes du haut et du bas. Toutes les flèches étant des isomorphismes, le diagramme de devant est donc commutatif. En écrivant les deux autres diagrammes analogues à ce dernier, on vérifie la condition de cocycle.

ii) Par hypothèse, il existe, pour tout $\alpha \in \Lambda$, un isomorphisme $\iota_{\alpha}: E_{\alpha} \stackrel{\sim}{\longrightarrow}$ $u_{\alpha K}^{*}\left(\left.E\right|_{]_{\alpha}\left[\mathcal{P}_{\alpha}\right.}\right)$, ceux-ci étant compatibles aux données de recollement respectives. Notons $\widetilde{\eta}_{\alpha \beta}$, la donnée de recollement canonique de $\left(u_{\alpha K}^{*}\left(\left.E\right|_{X_{\alpha}\left[\mathcal{P}_{\alpha}\right.}\right)\right)_{\alpha \in \Lambda}$, 
et $\widetilde{\eta}_{\alpha \beta}^{\prime}$ celle que l'on déduit pour la famille $\left(j_{\alpha}^{\dagger} u_{\alpha K}^{*}\left(\left.E\right|_{]_{X_{\alpha}\left[\mathcal{P}_{\alpha}\right.}}\right)\right)_{\alpha \in \Lambda}$. Dans le diagramme suivant

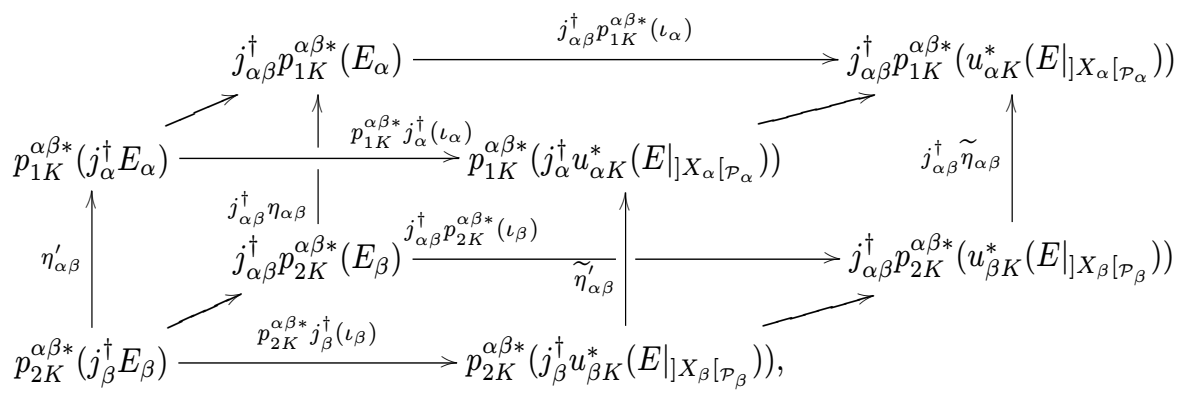

les carrés du fond, de droite et de gauche sont commutatifs par hypothèse ou définition, tandis que ceux du haut et du bas le sont par fonctorialité. Il en résulte que celui de devant l'est aussi. On obtient ainsi un isomorphisme $\left(\left(j_{\alpha}^{\dagger} E_{\alpha}\right)_{\alpha \in \Lambda},\left(\eta_{\alpha \beta}^{\prime}\right)_{\alpha, \beta \in \Lambda}\right) \stackrel{\sim}{\longrightarrow}\left(\left(j_{\alpha}^{\dagger} u_{\alpha K}^{*}\left(\left.E\right|_{] X_{\alpha}\left[\mathcal{P}_{\alpha}\right.}\right)\right)_{\alpha \in \Lambda},\left(\widetilde{\eta}_{\alpha \beta}^{\prime}\right)_{\alpha, \beta \in \Lambda}\right)$.

iii) Il suffit alors de prouver que l'isomorphisme canonique $u_{\alpha K}^{*}\left(\left.j^{\dagger} E\right|_{]_{X_{\alpha}\left[\mathcal{P}_{\alpha}\right.}}\right) \stackrel{\sim}{\longrightarrow} j_{\alpha}^{\dagger} u_{\alpha K}^{*}\left(\left.E\right|_{]_{X_{\alpha}\left[\mathcal{P}_{\alpha}\right.}}\right)$ est compatible aux données de recollement respectives. Pour cela, il s'agit d'établir la commutativité du carré de gauche du rectangle du fond du diagramme

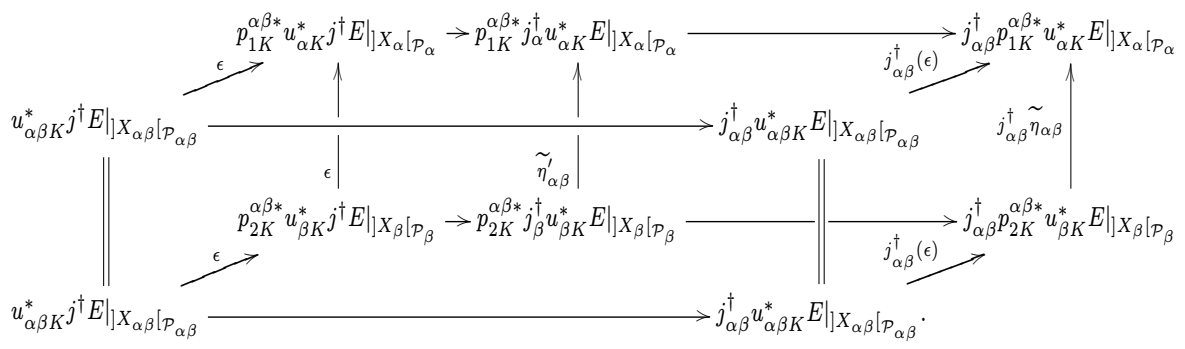

Le carré de droite du fond, les carrés de droite, de gauche et de devant sont commutatifs par définition. En outre, via 2.3.1.2 et par transitivité de l'isomorphisme de commutation des foncteurs de la forme $j^{\dagger}$ aux images inverses, il en est de même des rectangles du haut et du bas. Comme les flèches de 4.1.3.1 sont des isomorphismes, il en découle la commutativité du carré de gauche du fond.

LEMME 4.1.4. - Avec les notations 2.5.2 et de 2.5.4, soient $\left(\left(\mathcal{E}_{\alpha}\right)_{\alpha \in \Lambda}\right.$, $\left.\left(\theta_{\alpha \beta}\right)_{\alpha, \beta \in \Lambda}\right) \in \operatorname{Coh}\left(X,\left(\mathcal{P}_{\alpha}\right)_{\alpha \in \Lambda}\right)$ et $\mathcal{E} \in \operatorname{Coh}(X, \mathcal{P})$. Si $\operatorname{Recol}\left(\left(\mathcal{E}_{\alpha}\right)_{\alpha \in \Lambda}\right.$, $\left.\left(\theta_{\alpha \beta}\right)_{\alpha, \beta \in \Lambda}\right) \stackrel{\sim}{\longrightarrow} \mathcal{E}$ (voir les notations de la preuve de 2.5.4), alors $\operatorname{Recol}\left(\left(\left({ }^{\dagger} T \cap X_{\alpha}\right) \mathcal{E}_{\alpha}\right)_{\alpha \in \Lambda},\left(\theta_{\alpha \beta}^{\prime}\right)_{\alpha, \beta \in \Lambda}\right) \stackrel{\sim}{\longrightarrow}\left({ }^{\dagger} T\right)(\mathcal{E})$, où $\theta_{\alpha \beta}^{\prime}$ est l'unique 
isomorphisme rendant commutatif le diagramme :

$$
\begin{aligned}
& p_{1}^{\alpha \beta !}\left(\left({ }^{\dagger} T \cap X_{\alpha}\right)\left(\mathcal{E}_{\alpha}\right)\right) \longrightarrow\left({ }^{\dagger} T \cap X_{\alpha \beta}\right) \circ p_{1}^{\alpha \beta !}\left(\mathcal{E}_{\alpha}\right) \\
& \theta_{\alpha \beta}^{\prime} \wedge \quad\left({ }^{\dagger} T \cap X_{\alpha \beta}\right)\left(\theta_{\alpha \beta}\right) \uparrow \sim \\
& p_{2}^{\alpha \beta !}\left(\left({ }^{\dagger} T \cap X_{\beta}\right)\left(\mathcal{E}_{\beta}\right)\right) \longrightarrow\left({ }^{\dagger} T \cap X_{\alpha \beta}\right) \circ p_{2}^{\alpha \beta !}\left(\mathcal{E}_{\beta}\right),
\end{aligned}
$$

où les isomorphismes horizontaux résultent de [9, 2.2.18.1].

Démonstration. - Analogue à 4.1.3 en remplaçant l'utilisation de la commutativité du carré de gauche de 2.3.1.2 par celle de 2.1.11.

LEMME 4.1.5. - Soit $\left(\left(E_{\alpha}\right)_{\alpha \in \Lambda},\left(\eta_{\alpha \beta}\right)_{\alpha, \beta \in \Lambda}\right) \in \operatorname{Isoc}^{\dagger}\left(X, X,\left(\mathcal{P}_{\alpha}\right)_{\alpha \in \Lambda} / K\right)$. On note, via 4.1.3 puis 2.5.9.1, $\eta_{\alpha \beta}^{\prime}\left(\right.$ resp. $\left.\theta_{\alpha \beta}^{\prime \prime}\right)$ les isomorphismes canoniques de recollement de $\left(j_{\alpha}^{\dagger} E_{\alpha}\right)_{\alpha \in \Lambda}$ (resp. $\left.\left(\operatorname{sp}_{*} j_{\alpha}^{\dagger} E_{\alpha}\right)_{\alpha \in \Lambda}\right)$, tandis que ceux de $\left(\operatorname{sp}_{*} E_{\alpha}\right)_{\alpha \in \Lambda}$ (resp. $\left.\left(\left({ }^{\dagger} T \cap X_{\alpha}\right) \operatorname{sp}_{*} E_{\alpha}\right)_{\alpha \in \Lambda}\right)$ seront notés $\theta_{\alpha \beta}$ (resp. $\left.\theta_{\alpha \beta}^{\prime}\right)$.

On a l'isomorphisme

$$
\left.\left(\left(\operatorname{sp}_{*} j_{\alpha}^{\dagger} E_{\alpha}\right)_{\alpha \in \Lambda},\left(\theta_{\alpha \beta}^{\prime \prime}\right)_{\alpha, \beta \in \Lambda}\right) \stackrel{\sim}{\longrightarrow}\left(\left({ }^{\dagger} T \cap X_{\alpha}\right)\left(\operatorname{sp}_{*} E_{\alpha}\right)\right)_{\alpha \in \Lambda},\left(\theta_{\alpha \beta}^{\prime}\right)_{\alpha, \beta \in \Lambda}\right) .
$$

Démonstration. - Il s'agit de vérifier la commutativité du carré de droite du diagramme suivant :

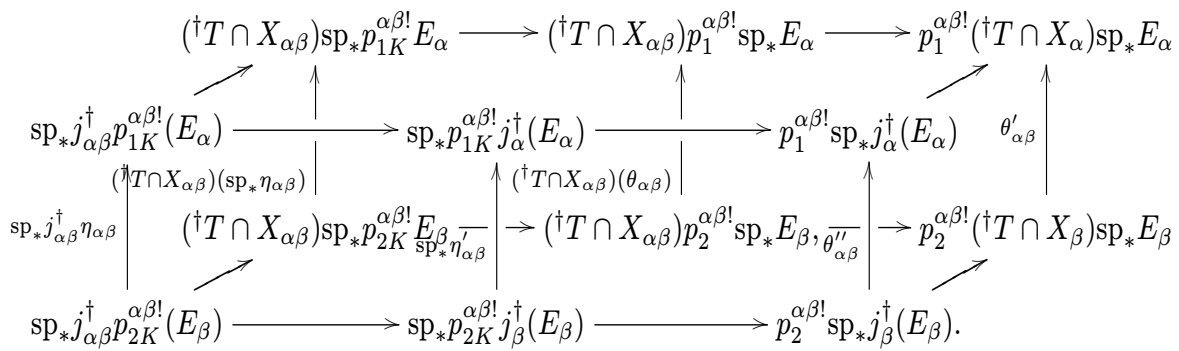

Le carré de gauche est commutatif par fonctorialité. Ceux de devant et de derrière le sont par définition. De plus, grâce à 2.4.1 (les foncteurs de la forme $j^{\dagger}$ et $\left({ }^{\dagger} T\right)$ sont respectivement des cas particuliers d'images inverses et d'images inverses extraordinaires), les rectangles du haut et du bas sont commutatifs. Le carré de droite de 4.1.5.1 l'est donc aussi.

Proposition 4.1.6. - Soit le diagramme commutatif

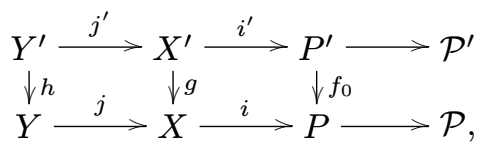

où $\mathcal{P}$ et $\mathcal{P}^{\prime}$ sont des $\mathcal{V}$-schémas formels lisses, $j$ et $j^{\prime}$ sont des immersions ouvertes de $k$-schémas lisses, $i$ et $i^{\prime}$ sont des immersions fermées. 
Le foncteur image inverse $g^{*}: \operatorname{Isoc}^{\dagger}(Y, X / K) \rightarrow \operatorname{Isoc}^{\dagger}\left(Y^{\prime}, X^{\prime} / K\right)$, défini dans [3, 2.3.2.(iv)], est canoniquement isomorphisme au foncteur $f_{0 K}^{*}$ de 2.3.1.

Démonstration. - Dans un premier temps, supposons que $X^{\prime}=X, Y^{\prime}=Y$ et que $f_{0}$ soit une immersion fermée. Notons $\delta_{f_{0}}: P^{\prime} \hookrightarrow P^{\prime} \times P$ le graphe de $f_{0}, p_{1}: \mathcal{P}^{\prime} \times_{\mathcal{S}} \mathcal{P} \rightarrow \mathcal{P}^{\prime}$ et $p_{2}: \mathcal{P}^{\prime} \times \mathcal{S} \mathcal{P} \rightarrow \mathcal{P}$ les projections canoniques. Soient $E$ un $j^{\dagger} \mathcal{O}_{]_{X}\left[_{\mathcal{P}}\right.}$-module à connexion intégrable et surconvergente et $E^{\prime}$ le $j^{\dagger} \mathcal{O}_{]_{\left[\mathcal{P}^{\prime}\right.}}$-module à connexion intégrable et surconvergente correspondant (voir [3, 2.3.2(i)]). Il s'agit de prouver l'isomorphisme : $E^{\prime} \stackrel{\sim}{\longrightarrow} f_{0 K}^{*}(E)$. Or, par construction, $p_{1 K}^{*}\left(E^{\prime}\right) \stackrel{\sim}{\longrightarrow} p_{2 K}^{*}(E)$. Il en dérive $\delta_{f_{0}, K}^{*} p_{1 K}^{*}\left(E^{\prime}\right) \stackrel{\sim}{\longrightarrow} \delta_{f_{0}, K}^{*} p_{2 K}^{*}(E)$, ce qui se réécrit $E^{\prime} \stackrel{\sim}{\longrightarrow} f_{0 K}^{*}(E)$.

De plus, le cas où le morphisme $f_{0}$ se relève en un morphisme $f: \mathcal{P}^{\prime} \rightarrow \mathcal{P}$ est immédiat. Celui où $X^{\prime}=X$ et $f_{0}$ est une immersion est donc aussi résolu.

Passons à présent au cas général. Le morphisme $f_{0}$ est le composé de l'immersion fermée $\delta_{f_{0}}$ suivi de la projection $P^{\prime} \times P \rightarrow P$ dont $\mathcal{P}^{\prime} \times \mathcal{S} \mathcal{P} \rightarrow \mathcal{P}$ constitue un relèvement. Les cas déjà traités nous permettent de conclure.

Avant de prouver la proposition suivante, nous aurons besoin de la remarque ci-après.

Remarques 4.1.7. - Le foncteur $\mathcal{L} o c$ construit dans la preuve de 2.5.4, s'étend, avec ses notations, en un foncteur de la catégorie $D_{\text {coh }}^{\mathrm{b}}\left(\mathcal{D}_{\mathcal{P}}^{\dagger}\left({ }^{\dagger} T\right)_{\mathbb{Q}}\right)$ dans celle des familles $\left(\left(\mathcal{E}_{\alpha}\right)_{\alpha \in \Lambda},\left(\theta_{\alpha \beta}\right)_{\alpha, \beta \in \Lambda}\right)$, où $\mathcal{E}_{\alpha} \in D_{\text {coh }}^{\mathrm{b}}\left(\mathcal{D}_{\mathfrak{X}_{\alpha}}^{\dagger}\left({ }^{\dagger} T \cap X_{\alpha}\right) \mathbb{Q}\right)$ et $\theta_{\alpha \beta}: p_{2}^{\alpha \beta !}\left(\mathcal{E}_{\beta}\right) \stackrel{\sim}{\longrightarrow} p_{1}^{\alpha \beta !}\left(\mathcal{E}_{\alpha}\right)$ sont des isomorphismes $\mathcal{D}_{\mathfrak{X}_{\alpha \beta}}^{\dagger}\left({ }^{\dagger} T \cap X_{\alpha \beta}\right)_{\mathbb{Q}^{-}}$ linéaires vérifiant la condition de cocycle $\theta_{13}^{\alpha \beta \gamma}=\theta_{12}^{\alpha \beta \gamma} \circ \theta_{23}^{\alpha \beta \gamma}$, où $\theta_{12}^{\alpha \beta \gamma}, \theta_{23}^{\alpha \beta \gamma}$ et $\theta_{23}^{\alpha \beta \gamma}$ sont définis par les diagrammes commutatifs analogues à 2.5.2.1.

Si $\mathcal{E} \in D_{\text {coh }}^{\mathrm{b}}\left(\mathcal{D}_{\mathcal{P}}^{\dagger}\left({ }^{\dagger} T\right)_{\mathbb{Q}}\right)$, le morphisme canonique $\mathcal{L} o c\left(\mathbb{R} \underline{\Gamma}_{X}^{\dagger} \mathcal{E}\right) \rightarrow \mathcal{L}$ oc $(\mathcal{E})$ est un isomorphisme.

Proposition 4.1.8. - On garde les notations et hypothèses de 4.1.6 et on suppose qu'il existe un diviseur $T$ de $P$ tel que $T^{\prime}:=f_{0}^{-1}(T)$ (resp. $T \cap X$ et $T^{\prime} \cap X^{\prime}$ ) soit un diviseur de $P^{\prime}$ (resp. $X$ et $X^{\prime}$ ) et tel que $Y=X \backslash T$ et $Y^{\prime}=X^{\prime} \backslash T^{\prime}$.

Pour tout isocristal $E$ sur $Y$ surconvergent le long de $T \cap X$, on dispose de l'isomorphisme :

$$
\operatorname{sp}_{X^{\prime} \hookrightarrow \mathcal{P}^{\prime}, T^{\prime}+} f_{0 K}^{*}(E) \stackrel{\sim}{\longrightarrow} \mathbb{R} \underline{\Gamma}_{X^{\prime}}^{\dagger} f_{0}^{!} \operatorname{sp}_{X \hookrightarrow \mathcal{P}, T+}(E)\left[-d_{X^{\prime} / X}\right]
$$

fonctoriel en $E$. 
Démonstration. - Comme $X$ et $X^{\prime}$ sont sommes directes de leurs composantes irréductibles, on se ramène au cas où $X$ et $X^{\prime}$ sont intègres. Traitons à présent le cas où $\mathcal{P}$ et $\mathcal{P}^{\prime}$ sont affines. Comme $f_{0}$ se relève et, pour tout relèvement $i^{\prime}$ : $\mathfrak{X}^{\prime} \hookrightarrow \mathcal{P}^{\prime}$ de l'immersion fermée $X^{\prime} \hookrightarrow P^{\prime}$, grâce à l'isomorphisme $\mathbb{R} \underline{\Gamma}_{X^{\prime}}^{\dagger} \stackrel{\sim}{\longrightarrow}$ $i_{+}^{\prime} \circ i^{\prime}$, ce cas est validé.

Puisque cela est local en $\mathcal{P}$ et $\mathcal{P}^{\prime}$, il résulte du premier cas que $\mathbb{R} \underline{\Gamma}_{X^{\prime}}^{\dagger} f_{0}^{!} \operatorname{sp}_{X \hookrightarrow \mathcal{P}, T+}(E)\left[-d_{X^{\prime} / X}\right]$ est un $\mathcal{D}_{\mathcal{P}^{\prime}}^{\dagger}\left({ }^{\dagger} T^{\prime}\right)_{\mathbb{Q}^{-}}$module cohérent à support dans $X^{\prime}$. Via 2.5.4, il suffit alors de prouver que l'isomorphisme $\mathcal{L} o c\left(\operatorname{sp}_{X^{\prime} \hookrightarrow \mathcal{P}^{\prime}, T^{\prime}+} f_{0 K}^{*}(E)\right) \stackrel{\sim}{\longrightarrow} \mathcal{L} \operatorname{Loc}\left(\mathbb{R}_{\Gamma_{X^{\prime}}^{\dagger}}^{\dagger} f_{0}^{!} \operatorname{sp}_{X \hookrightarrow \mathcal{P}, T+}(E)\left[-d_{X^{\prime} / X}\right]\right) \quad($ notations de 2.5.4).

À cette fin, choisissons une application surjective $\rho: \Lambda^{\prime} \rightarrow \Lambda$, deux recouvrements ouverts affines $\left(\mathcal{P}_{\alpha}\right)_{\alpha \in \Lambda}$ de $\mathcal{P}$ et $\left(\mathcal{P}_{\alpha^{\prime}}^{\prime}\right)_{\alpha^{\prime} \in \Lambda^{\prime}}$ de $\mathcal{P}^{\prime}$ tels que $f_{0}$ se factorise par $P_{\alpha^{\prime}}^{\prime} \rightarrow P_{\rho\left(\alpha^{\prime}\right)}$. On note alors $X_{\alpha}:=X \cap P_{\alpha}, X_{\alpha \beta}:=X \cap P_{\alpha} \cap P_{\beta}, j_{\alpha}$ : $\mathcal{P}_{\alpha} \subset \mathcal{P}$, et de même en rajoutant des primes. De plus, choisissons des relèvements $f_{\alpha^{\prime}}: \mathcal{P}_{\alpha^{\prime}}^{\prime} \rightarrow \mathcal{P}_{\rho\left(\alpha^{\prime}\right)}, i_{\alpha}: \mathfrak{X}_{\alpha} \hookrightarrow \mathcal{P}_{\alpha}, i_{\alpha^{\prime}}^{\prime}: \mathfrak{X}_{\alpha}^{\prime} \hookrightarrow \mathcal{P}_{\alpha^{\prime}}^{\prime}$ et $g_{\alpha^{\prime}}: \mathfrak{X}_{\alpha^{\prime}}^{\prime} \rightarrow \mathfrak{X}_{\rho\left(\alpha^{\prime}\right)}$ des relèvements des factorisations induites par $f_{0}, i, i^{\prime}$ et $g$.

Grâce à $4.1 .7, \quad \mathcal{L} o c\left(\mathbb{R} \underline{\Gamma}_{X^{\prime}}^{\dagger} f_{0}^{!} \operatorname{sp}_{X \hookrightarrow \mathcal{P}, T+}(E)\right) \quad \rightarrow \quad \mathcal{L} o c\left(f_{0}^{!} \operatorname{sp}_{X \hookrightarrow \mathcal{P}, T+}(E)\right)$ est un isomorphisme. Il suffit ainsi d'établir $\operatorname{Loc}\left(\operatorname{sp}_{X^{\prime} \hookrightarrow \mathcal{P}^{\prime}, T^{\prime}+} f_{0 K}^{*}(E)\right) \stackrel{\sim}{\longrightarrow}$ $\mathcal{L}$ oc $\left(f_{0}^{!} \operatorname{sp}_{X \hookrightarrow \mathcal{P}, T+}(E)\left[-d_{X^{\prime} / X}\right]\right)$.

L'objet $\quad \mathcal{L} o c\left(\operatorname{sp}_{X^{\prime} \hookrightarrow \mathcal{P}^{\prime}, T^{\prime}+} f_{0 K}^{*}(E)\right) \quad$ correspond à la famille $\left(\operatorname{sp}_{*} i_{\alpha^{\prime} K}^{\prime *} f_{\alpha^{\prime} K}^{*}\left(\left.E\right|_{] X_{\rho\left(\alpha^{\prime}\right)}\left[\mathcal{P}_{\rho\left(\alpha^{\prime}\right)}\right.}\right)\right)_{\alpha^{\prime} \in \Lambda^{\prime}}$, dont les isomorphismes de recollement sont induits par ceux de la forme $\epsilon$ (voir 2.3.1.1) et $\mathcal{L} o c\left(f_{0}^{!} \operatorname{sp}_{X \hookrightarrow \mathcal{P}, T+}(E)\right)$ est isomorphe à la famille $\left(i_{\alpha^{\prime}}^{\prime} j_{\alpha^{\prime}}^{\prime !} f_{0}^{!} \operatorname{sp}_{X \hookrightarrow \mathcal{P}, T+}(E)\left[-d_{X^{\prime} / X}\right]\right)_{\alpha^{\prime} \in \Lambda^{\prime}}$ dont les isomorphismes de recollement sont induits par ceux de la forme $\tau$.

Comme $i_{\alpha^{\prime}}^{\prime !} j_{\alpha^{\prime}}^{\prime !} f_{0}^{!} \stackrel{\sim}{\longrightarrow} i_{\alpha^{\prime}}^{\prime !} f_{\alpha^{\prime}}^{!} j_{\rho\left(\alpha^{\prime}\right)}^{!} \stackrel{\sim}{\longrightarrow} g_{\alpha^{\prime}}^{!} i_{\rho\left(\alpha^{\prime}\right)}^{!} j_{\rho\left(\alpha^{\prime}\right)}^{!}, \mathcal{L}$ oc $\left(f_{0}^{!} \operatorname{sp}_{X \hookrightarrow \mathcal{P}, T+}(E)\right)$ correspond à la famille $\left(g_{\alpha^{\prime}}^{!} i_{\rho\left(\alpha^{\prime}\right)}^{!} j_{\rho\left(\alpha^{\prime}\right)}^{!} \operatorname{sp}_{X \hookrightarrow \mathcal{P}, T+}(E)\left[-d_{X^{\prime} / X}\right]\right)_{\alpha^{\prime} \in \Lambda^{\prime}}$ dont les isomorphismes de recollement sont induits par ceux de la forme $\tau$. Puisque $\mathcal{L}$ oc $\circ \mathrm{sp}_{X \hookrightarrow \mathcal{P}, T+}(E) \stackrel{\sim}{\longrightarrow} \operatorname{sp}_{*} \mathcal{L}$ oc $(E)$ puis grâce à 2.4.6, on obtient $\left.g_{\alpha^{\prime}}^{!} i_{\rho\left(\alpha^{\prime}\right)}^{!} j_{\rho\left(\alpha^{\prime}\right)}^{!} \operatorname{sp}_{X \hookrightarrow \mathcal{P}, T+}(E)\right)\left[-d_{X^{\prime} / X}\right] \stackrel{\sim}{\longrightarrow} g_{\alpha^{\prime}}^{!} \operatorname{sp}_{*} i_{\rho\left(\alpha^{\prime}\right) K}^{*}\left(\left.E\right|_{] X_{\rho\left(\alpha^{\prime}\right)}\left[\mathcal{P}_{\rho\left(\alpha^{\prime}\right)}\right.}\right)\left[-d_{X^{\prime} / X}\right] \stackrel{\sim}{\longrightarrow}$ $\operatorname{sp}_{*} g_{\alpha^{\prime} K}^{*} i_{\rho\left(\alpha^{\prime}\right) K}^{*}\left(\left.E\right|_{X_{\rho\left(\alpha^{\prime}\right)}\left[\mathcal{P}_{\rho\left(\alpha^{\prime}\right)}\right.}\right)$, ceux-ci étant compatibles aux données de recollement respectives. On conclut ensuite via l'isomorphisme canonique $\epsilon$ : $i_{\rho\left(\alpha^{\prime}\right) K}^{*} \stackrel{\sim}{\longrightarrow} i_{\alpha^{\prime} K}^{*} f_{\alpha^{\prime} K}^{*}$.

Corollaire 4.1.9. - (i) Pour tout isocristal $E$ sur $Y$ surconvergent le long de $T_{X}$, on dispose d'un isomorphisme $\operatorname{sp}_{X \hookrightarrow \mathcal{P}, T+}\left(F^{*} E\right) \stackrel{\sim}{\longrightarrow} F^{*} \operatorname{sp}_{X \hookrightarrow \mathcal{P}, T+}(E)$ fonctoriel en $E$. On notera $F-\operatorname{Coh}(X, \mathcal{P}, T)$, la catégorie des $F-\mathcal{D}_{\mathcal{P}}^{\dagger}\left({ }^{\dagger} T\right)_{\mathbb{Q}^{-}}$ modules cohérents à support dans $X$. 
(ii) Le foncteur $\operatorname{sp}_{X \hookrightarrow \mathcal{P}, T+}: F-\operatorname{Isoc}^{\dagger}(Y, X / K) \rightarrow F-\operatorname{Coh}(X, \mathcal{P}, T)$ est pleinement fidèle et son image essentielle est constituée par les $F-\mathcal{D}_{\mathcal{P}}^{\dagger}\left({ }^{\dagger} T\right)_{\mathbb{Q}^{-}}$ modules cohérents $\mathcal{E}$ à support dans $X$ satisfaisant la condition suivante :

$\left(^{*}\right)$ pour tout ouvert $\mathcal{P}^{\prime}$ de $\mathcal{P}$ tel que l'immersion fermée $X \cap P^{\prime} \hookrightarrow P^{\prime}$ se relève en un morphisme $v: \mathfrak{X}^{\prime} \hookrightarrow \mathcal{P}^{\prime}$ de $\mathcal{V}$-schémas formels lisses, le faisceau $v^{!}\left(\left.\mathcal{E}\right|_{\mathcal{P}^{\prime}}\right)$ est $\mathcal{O}_{\mathfrak{X}^{\prime}, \mathbb{Q}}\left({ }^{\dagger} T \cap X^{\prime}\right)$-cohérent.

Démonstration. - L'isomorphisme $\operatorname{sp}_{X \hookrightarrow \mathcal{P}, T+}\left(F^{*} E\right) \stackrel{\sim}{\longrightarrow} F^{*} \operatorname{sp}_{X \hookrightarrow \mathcal{P}, T+}(E)$ résulte de 4.1.6 et de 4.1.8. En effet, pour tout $\mathcal{D}_{\mathcal{P}}^{\dagger}\left({ }^{\dagger} T\right)_{\mathbb{Q}^{-} \text {-module cohérent }}$ $\mathcal{E}$ à support dans $X$, le faisceau $F^{*}(\mathcal{E})$ est aussi à support dans $X$ et donc $\mathbb{R} \underline{\Gamma}_{X}^{\dagger} F^{*}(\mathcal{E}) \stackrel{\sim}{\longrightarrow} F^{*}(\mathcal{E})$. Le foncteur $\operatorname{sp}_{X \hookrightarrow \mathcal{P}, T+}$ induit donc le suivant $F$ $\operatorname{Isoc}^{\dagger}(Y, X / K) \rightarrow F-\operatorname{Coh}(X, \mathcal{P}, T)$. La fidélité de celui-ci résulte du cas sans structure de Frobenius (2.5.10). Pour la pleine fidélité, soient $E$ et $E^{\prime}$ deux objets de $F$-Isoc $\operatorname{Is}^{\dagger}(Y, X / K)$ et $g$ un morphisme $\operatorname{sp}_{X \hookrightarrow \mathcal{P}, T+}(E) \rightarrow \operatorname{sp}_{X \hookrightarrow \mathcal{P}, T+}\left(E^{\prime}\right)$ commutant à Frobenius. D'après 2.5.10, il existe un morphisme $f: E \rightarrow E^{\prime}$ dans $\operatorname{Isoc}^{\dagger}(Y, X / K)$ tel que $\operatorname{sp}_{X \hookrightarrow \mathcal{P}, T+}(f)=g$. Puisque le foncteur $\operatorname{sp}_{X \hookrightarrow \mathcal{P}, T+}$ (sans structure de Frobenius) est fidèle, le morphisme $f$ commute à Frobenius. On a donc vérifié la pleine fidélité. Enfin, la description de l'image essentielle découle de celle de 2.5.10 ainsi que de la pleine fidélité de $\operatorname{sp}_{X \hookrightarrow \mathcal{P}, T+}$ (sans structure de Frobenius).

Remarques 4.1.10. - Les isomorphismes 4.1 .8 et 4.1.2 sont compatibles à Frobenius.

\subsection{Isomorphisme de commutation des foncteurs duaux aux images inverses extra-} ordinaires par une immersion. - Cette section est dédiée à l'établissement du résultat suivant : soient $f: \mathfrak{X}^{\prime} \hookrightarrow \mathfrak{X}$ une immersion de $\mathcal{V}$-schémas formels lisses, $T$ un diviseur de $X$ tel que $T^{\prime}:=f^{-1}(T)$ soit un diviseur de $X^{\prime}$, $\mathcal{E} \in D_{\text {coh }}^{\mathrm{b}}\left({ }^{\mathrm{g}} \mathcal{D}_{\mathfrak{X}}^{\dagger}\left({ }^{\dagger} T\right)_{\mathbb{Q}}\right)$ à support dans l'adhérence schématique $\overline{X^{\prime}}$ de $X^{\prime}$ dans $X$. On construit, via 4.2.5.1, un isomorphisme canonique $\theta_{f, T, \mathcal{E}}: f_{T}^{!} \mathbb{D}_{T}(\mathcal{E}) \stackrel{\sim}{\longrightarrow}$ $\mathbb{D}_{T^{\prime}} f_{T}^{!}(\mathcal{E})$. Celui-ci est en outre transitif pour la composition d'immersions (voir 4.2.8).

4.2.1. - Soient $g: \mathfrak{X}^{\prime} \hookrightarrow \mathfrak{X}$ une immersion fermée de $\mathcal{V}$-schémas formels lisses, $T$ un diviseur de $X$ tel que $T^{\prime}:=T \cap X^{\prime}$ soit un diviseur de $X^{\prime}$. Pour tout $\mathcal{E} \in D_{\text {coh }}^{\mathrm{b}}\left({ }^{\mathrm{g}} \mathcal{D}_{\mathfrak{X}, \mathbb{Q}}^{\dagger}\left({ }^{\dagger} T\right)\right)$ à support dans $X^{\prime}$, on définit l'isomorphisme $\theta_{g, T, \mathcal{E}}$ : $g_{T}^{!} \circ \mathbb{D}_{T}(\mathcal{E}) \stackrel{\sim}{\longrightarrow} \mathbb{D}_{T^{\prime}} \circ g_{T}^{!}(\mathcal{E})$ via le diagramme commutatif suivant :

$$
\begin{aligned}
& g_{T}^{!} \mathbb{D}_{T} g_{T,+} g_{T}^{!}(\mathcal{E}) \longleftarrow \frac{g_{T}^{!} \chi_{g} g_{T}^{!}}{\sim} g_{T}^{!} g_{T,+} \mathbb{D}_{T^{\prime}} g_{T}^{!}(\mathcal{E})
\end{aligned}
$$

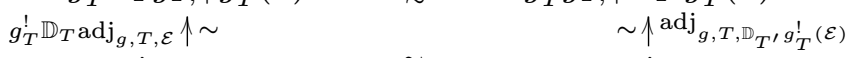

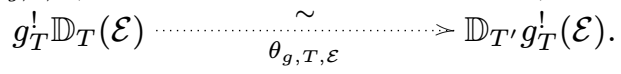


On pourra le noter plus simplement $\theta_{g, T}$ ou $\theta_{g, \mathcal{E}}$ voire $\theta_{g}$.

4.2.2. - Avec les notations de 4.2.1, on aurait pu définir d'une seconde façon, via le diagramme commutatif 4.2.2.2, l'isomorphisme $\theta_{g, T, \mathcal{E}}$ : pour vérifier la commutativité de 4.2.2.2, on remarque d'abord que les contours des deux diagrammes ci-dessous :

$$
\begin{gathered}
g_{T,+} g_{T}^{!} \mathbb{D}_{T}(\mathcal{E}) \stackrel{\operatorname{adj}_{g}}{\sim} \underset{\sim}{\sim} g_{T,+} g_{T}^{!} \mathbb{D}_{T} g_{T,+} g_{T}^{!}(\mathcal{E}) \stackrel{\operatorname{adj}_{g}}{\sim} \mathbb{D}_{T} g_{T,+} g_{T}^{!}(\mathcal{E}) \\
\sim \downarrow \theta_{g} \\
g_{T,+} \mathbb{D}_{T^{\prime}} g_{T}^{!}(\mathcal{E}) \stackrel{\sim}{\stackrel{\operatorname{adj}_{g}}{\sim}} \underset{\sim}{\sim} g_{T,+} g_{T}^{!} g_{T,+} \mathbb{D}_{T^{\prime}} g_{T}^{!}(\mathcal{E}) \stackrel{\operatorname{adj}_{g}}{\sim} g_{T,+} \mathbb{D}_{T^{\prime}} g_{T}^{!}(\mathcal{E}),
\end{gathered}
$$

$$
\begin{gathered}
g_{T,+} g_{T}^{!} \mathbb{D}_{T}(\mathcal{E}) \stackrel{\operatorname{adj}_{g}}{\sim} \mathbb{D}_{T}(\mathcal{E}) \stackrel{\operatorname{adj}_{g}}{\sim} \mathbb{D}_{T} g_{T,+} g_{T}^{!}(\mathcal{E}) \\
\sim \downarrow \theta_{g} \\
g_{T,+} \mathbb{D}_{T^{\prime}} g_{T}^{!}(\mathcal{E}) \stackrel{\sim \downarrow \chi_{g}}{=} g_{T,+} \mathbb{D}_{T^{\prime}} g_{T}^{!}(\mathcal{E}),
\end{gathered}
$$

sont égaux. En effet, les composés des morphismes du haut sont égaux par fonctorialité (on établit la commutativité du carré correspondant), tandis que pour ceux du bas, cela découle fonctoriellement d'une propriété standard des foncteurs adjoints. Or, le carré de gauche de 4.2.2.1 est l'image de 4.2.1.1 par $g_{T,+}$. La commutativité de celui de droite étant fonctorielle, il en résulte celle de 4.2.2.1. Ainsi, 4.2.2.2 est commutatif. Comme $g$ est une immersion fermée, d'après l'analogue $p$-adique de Berthelot du théorème de Kashiwara, la commutativité de 4.2.2.2 détermine $\theta_{g}$.

Proposition 4.2.3. - Soient $g, g^{\prime}: \mathfrak{X}^{\prime} \rightarrow \mathfrak{X}$ deux immersions fermées de $\mathcal{V}$-schémas formels lisses telles que $g_{0}=g_{0}^{\prime}$, $T$ un diviseur de $X$ tel que $T^{\prime}:=$ $T \cap X^{\prime}$ soit un diviseur de $X^{\prime}$. On dispose du diagramme commutatif :

$$
\begin{array}{r}
g_{T}^{!} \mathbb{D}_{T}(\mathcal{E}) \underset{\theta_{g, T, \mathcal{E}}}{\stackrel{\sim}{\longrightarrow}} \mathbb{D}_{T^{\prime}} g_{T}^{!}(\mathcal{E}) \\
\tau_{g^{\prime}, g \downarrow \sim \uparrow \tau_{g^{\prime}, g}} \sim \\
g_{T}^{\prime !} \mathbb{D}_{T}(\mathcal{E}) \underset{\theta_{g^{\prime}, T, \mathcal{E}}}{\sim} \mathbb{D}_{T^{\prime}} g_{T}^{\prime !}(\mathcal{E}) .
\end{array}
$$

Démonstration. - Considérons le diagramme suivant :

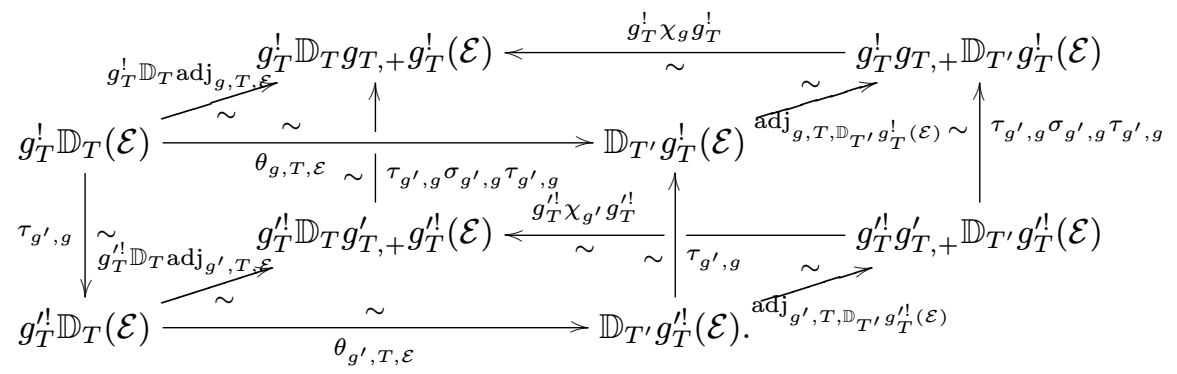


Il découle de [16, 2.4.4.2] et [16, 2.5.1] (ou [16, 2.6.2]) que les carrés de droite, de gauche et de derrière sont commutatifs. Comme les deux horizontaux le sont par définition (4.2.1.1), le dernier carré de 4.2.3.2, celui de devant, est donc commutatif.

Proposition 4.2.4. - Soient $g^{\prime}: \mathfrak{X}^{\prime \prime} \rightarrow \mathfrak{X}^{\prime}$ et $g: \mathfrak{X}^{\prime} \rightarrow \mathfrak{X}$ deux immersions fermées de $\mathcal{V}$-schémas formels lisses, $T$ un diviseur de $X$ tel que $T^{\prime}:=$ $g^{-1}(T)$ (resp. $\left.T^{\prime \prime}:=g^{\prime-1}\left(T^{\prime}\right)\right)$ soit un diviseur de $X^{\prime}$ (resp. $\left.X^{\prime \prime}\right)$. Pour tout $\mathcal{E} \in D_{\text {coh }}^{\mathrm{b}}\left({ }^{g} \mathcal{D}_{\mathfrak{X}, \mathbb{Q}}^{\dagger}\left({ }^{\dagger} T\right)\right)$ à support dans $X^{\prime \prime}$, le diagramme

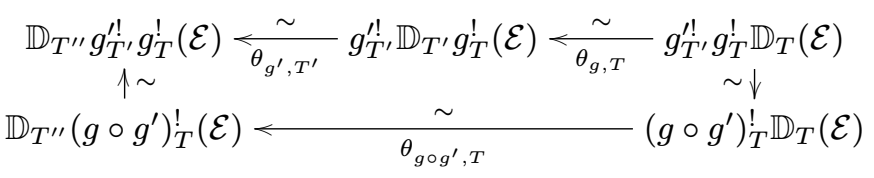

est commutatif.

Démonstration. - Quitte à alourdir les notations, on suppose que le diviseur $T$ est vide et on n'indiquera pas « $(\mathcal{E}) »$. Considérons le cube ci-dessous :

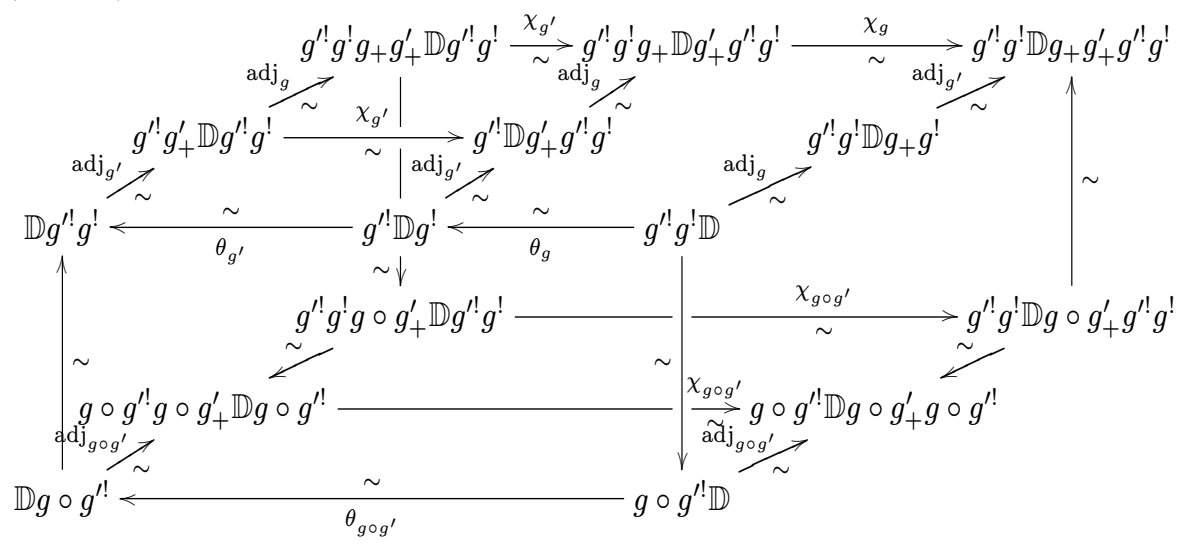

Le carré du fond de 4.2.4.1 correspond, en omettant les termes $g^{!}$et $g^{\prime !}$, au diagramme signifiant la transitivité des isomorphismes de la forme $\chi$. Celuici est donc commutatif. Par fonctorialité ou définition (4.2.1.1), on obtient la commutativité de la face du bas. On vérifie de même celle de la partie gauche de la face supérieure de 4.2.4.1. Sa partie droite s'identifie (i.e. les composés des contours respectifs sont égaux) au contour du diagramme commutatif

$$
\begin{aligned}
& g^{\prime !} \mathbb{D} g^{!} \stackrel{\operatorname{adj}_{g}}{\sim} g^{\prime} g^{!} g_{+} \mathbb{D} g^{!} \stackrel{\operatorname{adj}_{g^{\prime}}}{\sim} g^{\prime} g^{!} g_{+} \mathbb{D} g_{+}^{\prime} g^{\prime !} g^{!} \\
& \begin{array}{cc}
\theta_{g} \uparrow \sim & \sim \downarrow \chi \chi_{g} \\
g^{\prime !} g^{!} \mathbb{D} \stackrel{\operatorname{adj}_{g}}{\sim} g^{\prime} g^{!} \mathbb{D} g_{+} g^{!} \stackrel{\operatorname{adj}_{g^{\prime}}}{\sim} g^{\prime} g^{!} \mathbb{D} g_{+} g_{+}^{\prime} g^{\prime} g^{!} .
\end{array}
\end{aligned}
$$


La face du haut de 4.2.4.1 est donc commutative.

En appliquant $g^{\prime !} g ! \mathbb{D}$ au carré de gauche de 1.2.11 (en remplaçant $f$ et $g$ par $g$ et $g^{\prime}$ ), on obtient le rectangle supérieur de

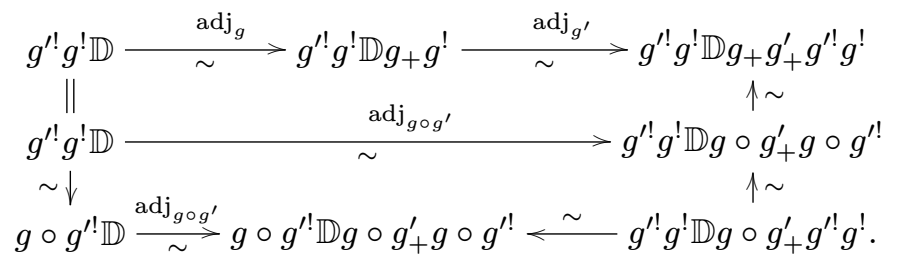

Le diagramme 4.2.4.2 est donc commutatif. Son contour, i.e., le carré de droite de 4.2.4.1 l'est donc aussi. De même, on obtient la commutativité du carré de gauche de 4.2.4.1.

Comme les flèches de 4.2.4.1 sont des isomorphismes, comme cinq des faces du cube de 4.2.4.1 sont commutatifs, le dernier, i.e., celui de devant, égal à celui de 4.2.4, est commutatif.

Lemme 4.2.5. - Soient $f: \mathfrak{X}^{\prime} \hookrightarrow \mathfrak{X}$ une immersion de $\mathcal{V}$-schémas formels lisses, $T$ un diviseur de $X$ tel que $T^{\prime}:=f^{-1}(T)$ soit un diviseur de $X^{\prime}, \mathcal{E} \in$ $D_{\mathrm{coh}}^{\mathrm{b}}\left({ }^{\mathrm{g}} \mathcal{D}_{\mathfrak{X}}^{\dagger}\left({ }^{\dagger} T\right)_{\mathbb{Q}}\right)$ à support dans l'adhérence schématique $\overline{X^{\prime}}$ de $X^{\prime}$ dans $X$.

Choisissons $\mathcal{Y}$ un ouvert de $\mathfrak{X}$ tel que $f$ se factorise en une immersion fermée $g: \mathfrak{X}^{\prime} \hookrightarrow \mathcal{Y}$. En notant $j: \mathcal{Y} \subset \mathfrak{X}$ l'inclusion, l'isomorphisme composé

$f_{T}^{!} \mathbb{D}_{T}(\mathcal{E}) \stackrel{\sim}{\longrightarrow} g_{T \cap Y}^{!} j_{T}^{!} \mathbb{D}(\mathcal{E})=g_{T \cap Y}^{!} \mathbb{D}_{T \cap Y} j_{T}^{!}(\mathcal{E}) \underset{\theta_{g}}{\sim} \mathbb{D}_{T^{\prime}} g_{T \cap Y}^{!} j_{T}^{!}(\mathcal{E}) \stackrel{\sim}{\longrightarrow} \mathbb{D}_{T^{\prime}} f_{T}^{!}(\mathcal{E})$

ne dépend pas du choix de la factorisation de $f$ par $g$ et $j$.

Démonstration. - Pour le vérifier, quitte à alourdir les notations, supposons le diviseur $T$ vide. Notons $\mathcal{Y}_{0}$ l'ouvert de $\mathfrak{X}$ dont l'espace sous-jacent est $X \backslash$ $\left(\overline{X^{\prime}} \backslash X^{\prime}\right)$. Comme $\mathcal{Y} \subset \mathcal{Y}_{0}$ et que le morphisme induit (par $g$ ) $\mathfrak{X}^{\prime} \hookrightarrow \mathcal{Y}_{0}$ est une immersion fermée, on remarque qu'il suffit de prouver cette indépendance lorsque $f$ est une immersion fermée. Supposons donc $f$ est une immersion fermée. Considérons le diagramme ci-dessous :

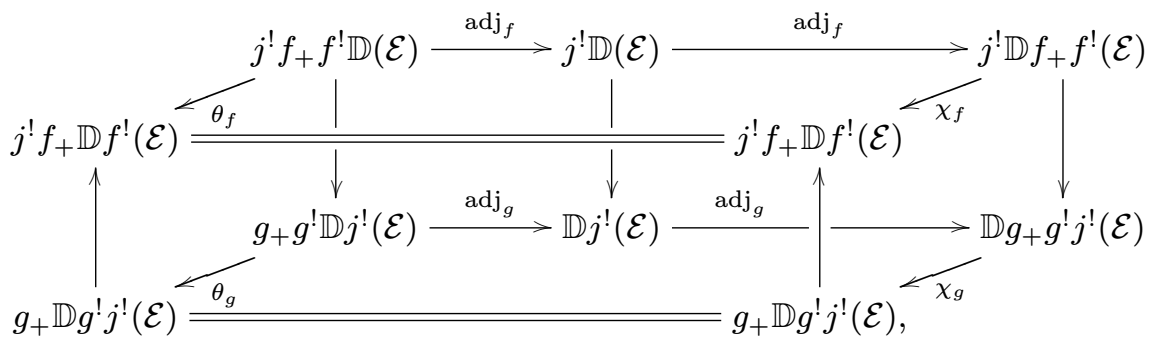

TOME $137-2009-\mathrm{N}^{\mathrm{O}} 4$ 
dont les flèches verticales se déduisent des isomorphismes canoniques $f^{!} \stackrel{\sim}{\longrightarrow}$ $g^{!} j^{!}$et $j^{!} f_{+} \stackrel{\sim}{\longrightarrow} g_{+}$. Il résulte de 4.2.2.2 que les faces horizontales sont commutatives. La commutativité des faces de devant, derrière et de droite se vérifie par construction de $\chi$ et adj. Comme toutes les flèches de 4.2.5.2 sont des isomorphismes, il en résulte celle du carré de gauche. Via l'isomorphisme canonique $j^{!} f_{+} \stackrel{\sim}{\longrightarrow} g_{+}$et grâce à l'analogue $p$-adique du théorème de Kashiwara, le carré de gauche de 4.2.5.2 dont on a enlevé les termes $j^{!} f_{+}$et $g_{+}$est alors commutatif. Cela se traduit par le fait que le composé 4.2.5.1 est $\theta_{f}$.

Notations 4.2.6. - Avec les notations de 4.2.5, on désignera par $\theta_{f, T, \mathcal{E}}$ : $f_{T}^{!} \mathbb{D}_{T}(\mathcal{E}) \stackrel{\sim}{\longrightarrow} \mathbb{D}_{T^{\prime}} f_{T}^{!}(\mathcal{E})$ l'isomorphisme 4.2.5.1.

Proposition 4.2.7. - Soient $f, f^{\prime}: \mathfrak{X}^{\prime} \hookrightarrow \mathfrak{X}$ deux immersions de $\mathcal{V}$-schémas formels lisses telles que $f_{0}=f_{0}^{\prime}, T$ un diviseur de $X$ tel que $T^{\prime}:=f_{0}^{-1}(T)$ soit un diviseur de $X^{\prime}$. On bénéficie du diagramme commutatif :

$$
\begin{aligned}
& f_{T}^{!} \mathbb{D}_{T}(\mathcal{E}) \underset{\theta_{f, T, \mathcal{E}}}{\stackrel{\sim}{\longrightarrow}} \mathbb{D}_{T^{\prime}} f_{T}^{!}(\mathcal{E}) \\
& \tau_{f^{\prime}, f} \sim \tau_{f^{\prime}, f} \\
& f_{T}^{\prime !} \mathbb{D}_{T}(\mathcal{E}) \underset{\theta_{f^{\prime}, T, \mathcal{E}}}{\sim} \mathbb{D}_{T^{\prime}} f_{T}^{\prime !}(\mathcal{E}) .
\end{aligned}
$$

Démonstration. - Notons $\mathcal{Y}_{0}$ l'ouvert de $\mathfrak{X}$ dont l'espace sous-jacent est $X \backslash$ $\left(\overline{X^{\prime}} \backslash X^{\prime}\right), j: \mathcal{Y}_{0} \subset \mathfrak{X}$ l'inclusion canonique, $g$ et $g^{\prime}: \mathfrak{X}^{\prime} \hookrightarrow \mathcal{Y}_{0}$ les immersions fermées factorisant respectivement $f$ et $f^{\prime}$. Il suffit de prouver que le diagramme $(4.2 .7 .2)$

$$
\begin{aligned}
& f_{T}^{!} \mathbb{D}_{T}(\mathcal{E}) \underset{\sim}{\sim} g_{T \cap Y}^{!} j_{T}^{!} \mathbb{D}(\mathcal{E})=g_{T \cap Y}^{!} \mathbb{D}_{T \cap Y} j_{T}^{!}(\mathcal{E}) \stackrel{\theta_{g}}{\sim} \mathbb{D}_{T^{\prime}} g_{T \cap Y}^{!} j_{T}^{!}(\mathcal{E}) \underset{\sim}{\sim} \mathbb{D}_{T^{\prime}} f_{T}^{!}(\mathcal{E})
\end{aligned}
$$

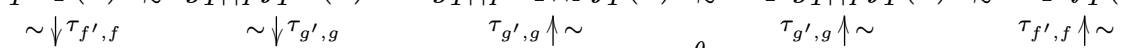

$$
\begin{aligned}
& f_{T}^{\prime !} \mathbb{D}_{T}(\mathcal{E}) \underset{\sim}{\sim} g_{T \cap Y}^{\prime !} j_{T}^{!} \mathbb{D}(\mathcal{E})=g_{T \cap Y}^{\prime !} \mathbb{D}_{T \cap Y} j_{T}^{!}(\mathcal{E}) \stackrel{\theta_{g}}{\sim} \mathbb{D}_{T^{\prime}} g_{T \cap Y}^{\prime !} j_{T}^{!}(\mathcal{E}) \underset{\sim}{\sim} \mathbb{D}_{T^{\prime}} f_{T}^{\prime !}(\mathcal{E})
\end{aligned}
$$

est commutatif. Or, grâce à 4.2.3, le deuxième carré de droite est commutatif. La commutativité du deuxième carré de gauche est évidente tandis que celle des deux autres se vérifie par transitivité des isomorphismes de la forme $\tau$.

Proposition 4.2.8. - Soient $f: \mathfrak{X}^{\prime} \hookrightarrow \mathfrak{X}$ et $f^{\prime}: \mathfrak{X}^{\prime \prime} \hookrightarrow \mathfrak{X}^{\prime}$ deux immersions de $\mathcal{V}$-schémas formels lisses, $T$ un diviseur de $X$ tel que $T^{\prime}:=f^{-1}(T)$ (resp. $T^{\prime \prime}:=f^{\prime-1}\left(T^{\prime}\right)$ soit un diviseur de $X^{\prime}$ (resp. $\left.X^{\prime \prime}\right)$. Pour tout $\mathcal{E} \in$ $D_{\text {coh }}^{\mathrm{b}}\left({ }^{\mathrm{g}} \mathcal{D}_{\mathfrak{X}}^{\dagger}\left({ }^{\dagger} T\right)_{\mathbb{Q}}\right)$ à support dans l'adhérence schématique de $X^{\prime \prime}$ dans $X$, le diagramme suivant est commutatif :

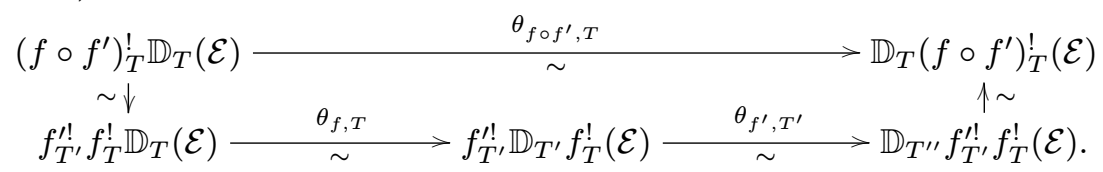


Démonstration. - Par [4, 4.3.12], on se ramène au cas où $T$ est vide. On désigne par $\mathcal{Y}$ (resp. $\mathcal{Y}^{\prime}$ ) un ouvert de $\mathfrak{X}$ (resp. $\mathfrak{X}^{\prime}$ ) tel que $f$ (resp. $f^{\prime}$ ) se factorise en une immersion fermée $g: \mathfrak{X}^{\prime} \hookrightarrow \mathcal{Y}$ (resp. $g^{\prime}: \mathfrak{X}^{\prime \prime} \hookrightarrow \mathcal{Y}^{\prime}$ ). Soit $\mathcal{Y}^{\prime \prime}$ un ouvert de $\mathcal{Y}$ tel que $g^{-1}\left(\mathcal{Y}^{\prime \prime}\right)=\mathcal{Y}^{\prime}$. On note $j: \mathcal{Y} \subset \mathfrak{X}$ et $j^{\prime}: \mathcal{Y}^{\prime} \subset \mathfrak{X}^{\prime}$ les inclusions canoniques. Par abus de notations, on désignera encore par $j^{\prime}$ : $\mathcal{Y}^{\prime \prime} \subset \mathcal{Y}$ et $g: \mathcal{Y}^{\prime} \hookrightarrow \mathcal{Y}^{\prime \prime}$ les morphismes canoniques. Considérons le diagramme : $(4.2 .8 .2)$

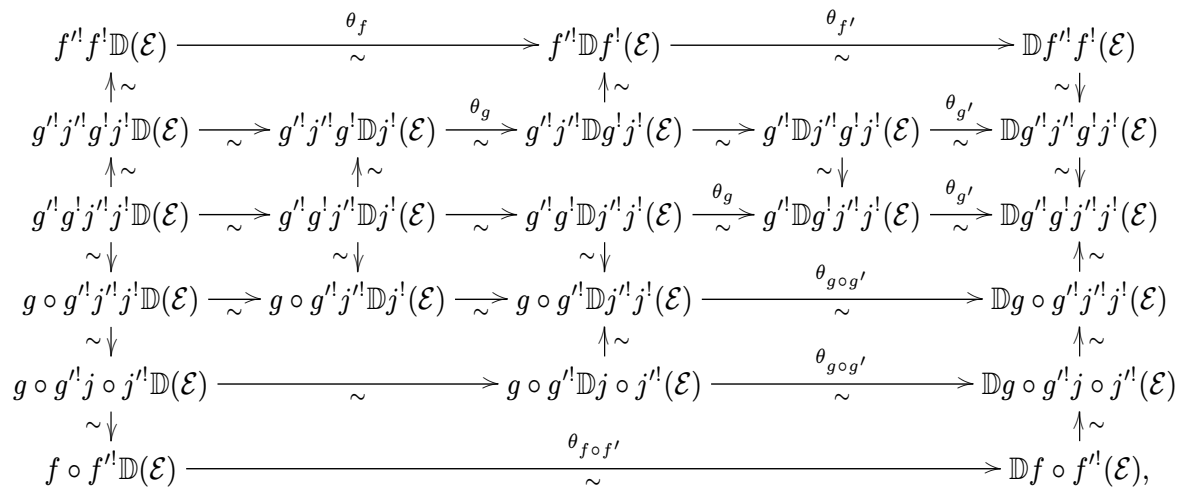

dont le contour correspond à celui de 4.2.8.1 (on vérifie par transitivité que le composé de droite de 4.2.8.2 est le morphisme de droite de 4.2.8.1, de même à gauche). Il dérive de 4.2.5 que les rectangles du haut et du bas sont commutatifs. La commutativité du rectangle de la deuxième ligne résulte du fait que les isomorphismes de la forme $\theta_{g}$ commute à la restriction à un ouvert. Celle du rectangle de la troisième ligne découle de 4.2.4 tandis que celle du rectangle de gauche de l'avant dernière ligne est immédiate. Celle des carrés se vérifie par fonctorialité. Le diagramme 4.2.8.2 est donc commutatif.

4.3. Commutation au foncteur dual. - Nous reprenons les notations de 2.5.1.

Proposition 4.3.1. - Désignons par $E$ un isocristal sur $Y$ surconvergent le long de $T_{X}$ et par $E^{\vee}$ son dual. On a l'isomorphisme canonique fonctoriel en $E: \operatorname{sp}_{X \hookrightarrow \mathcal{P}, T+}\left(E^{\vee}\right) \stackrel{\sim}{\longrightarrow} \mathbb{D}_{T} \circ \operatorname{sp}_{X \hookrightarrow \mathcal{P}, T+}(E)$.

Démonstration. - Comme d'habitude, on peut supposer $X$ intègre. En remplaçant les foncteurs de la forme $j^{\dagger}$ ou $\left({ }^{\dagger} T\right)$ par les foncteurs duaux respectifs, on procède de manière analogue à la preuve de 4.1.2. Par exemple, avec les notations de 2.5.4, grâce à 4.2.7 et 4.2.8, on vérifie pour tout $\mathcal{E} \in \operatorname{Coh}(X, \mathcal{P}, T)$ 
la commutativité des diagrammes ci-dessous :

$$
\begin{gathered}
u_{\alpha \beta}^{!} \mathbb{D}_{T \cap P_{\alpha \beta}}\left(\left.\mathcal{E}\right|_{\mathcal{P}_{\alpha \beta}}\right) \longrightarrow \mathbb{D}_{T \cap X_{\alpha \beta}} u_{\alpha \beta}^{!}\left(\left.\mathcal{E}\right|_{\mathcal{P}_{\alpha \beta}}\right) \\
\downarrow \mathbb{D}_{T \cap P_{\alpha \beta} \tau} \\
p_{1}^{\alpha \beta !} u_{\alpha}^{!} \mathbb{D}_{T \cap P_{\alpha}}\left(\left.\mathcal{E}\right|_{\mathcal{P}_{\alpha}}\right) \longrightarrow p_{1}^{\alpha \beta !} \mathbb{D}_{T \cap X_{\alpha}} u_{\alpha}^{!}\left(\left.\mathcal{E}\right|_{\mathcal{P}_{\alpha}}\right) \longrightarrow \mathbb{D}_{T \cap X_{\alpha \beta}} p_{1}^{\alpha \beta !} u_{\alpha}^{!}\left(\left.\mathcal{E}\right|_{\mathcal{P}_{\alpha}}\right),
\end{gathered}
$$

$$
\begin{aligned}
& u_{\alpha \beta}^{!} \mathbb{D}_{T \cap P_{\alpha \beta}}\left(\left.\mathcal{E}\right|_{\mathcal{P}_{\alpha \beta}}\right) \longrightarrow \mathbb{D}_{T \cap X_{\alpha \beta}} u_{\alpha \beta}^{!}\left(\left.\mathcal{E}\right|_{\mathcal{P}_{\alpha \beta}}\right) \\
& \downarrow \mathbb{D}_{T \cap P_{\alpha \beta} \tau} \quad \downarrow \tau \\
& p_{2}^{\alpha \beta !} u_{\beta}^{!} \mathbb{D}_{T \cap P_{\alpha}}\left(\left.\mathcal{E}\right|_{\mathcal{P}_{\beta}}\right) \longrightarrow p_{2}^{\alpha \beta !} \mathbb{D}_{T \cap X_{\beta}} u_{\beta}^{!}\left(\left.\mathcal{E}\right|_{\mathcal{P}_{\beta}}\right) \longrightarrow \mathbb{D}_{T \cap X_{\alpha \beta}} p_{2}^{\alpha \beta !} u_{\beta}^{!}\left(\left.\mathcal{E}\right|_{\mathcal{P}_{\beta}}\right),
\end{aligned}
$$

qui correspondent aux analogues des carrés horizontaux de 4.1.3.1. De plus, pour valider l'analogue du lemme 4.1.5, on utilise 3.3.13.

Notations 4.3.2. - Soient $\mathcal{E}^{(\bullet)}, \mathcal{F}^{(\bullet)} \in \underset{\rightarrow}{L D_{\mathbb{Q}, \mathrm{qc}}^{\mathrm{b}}}\left(\widehat{\mathcal{D}}_{\mathcal{P}}^{(\bullet)}(T)\right)$ (voir [12, 1.1]. On note

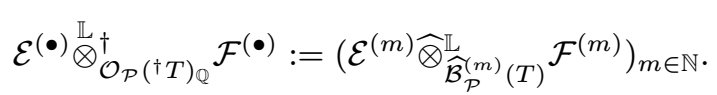

Pour une définition des images directes et inverses extraordinaires à singularités surconvergentes le long d'un diviseur de ces complexes quasi-cohérents, on pourra consulter [12, 1.1.7].

De manière analogue à $[6,4.2 .2]$, on dispose du foncteur $\stackrel{\lim }{\longrightarrow} \underset{L}{L} D_{\mathbb{Q}, \mathrm{qc}}^{\mathrm{b}}\left(\widehat{\mathcal{D}}_{\mathcal{P}}^{(\bullet \bullet}(T)\right) \rightarrow D\left(\mathcal{D}_{\mathcal{P}}^{\dagger}\left({ }^{\dagger} T\right)_{\mathbb{Q}}\right)$. Celui-ci induit une équivalence de catégorie entre $D_{\mathrm{coh}}^{\mathrm{b}}\left(\mathcal{D}_{\mathcal{P}}^{\dagger}\left({ }^{\dagger} T\right)_{\mathbb{Q}}\right)$ et une sous-catégorie pleine de $L D_{\mathbb{Q}, \mathrm{qc}}^{\mathrm{b}}\left(\widehat{\mathcal{D}}_{\mathcal{P}}^{(\bullet)}(T)\right)$,

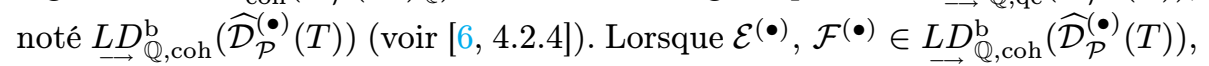
en notant $\mathcal{E}:=\lim \mathcal{E}^{(\bullet)}$ et $\mathcal{F}:=\lim \mathcal{F}^{(\bullet)}$, on pose

$$
\mathcal{E}^{\mathbb{L}}{ }_{\mathcal{O}_{\mathcal{P}}(\dagger T)_{\mathbb{Q}}}^{\dagger} \mathcal{F}:=\lim _{\longrightarrow} \mathcal{E}^{(\bullet)} \stackrel{\mathbb{L}}{\otimes}_{\mathcal{O}_{\mathcal{P}}(\dagger T)_{\mathbb{Q}}} \mathcal{F}^{(\bullet)}
$$

4.3.3. - L'isomorphisme d'autodualité de $[2,2.1 .1]$ s'écrit $\mathbb{D}_{\mathcal{P}, T}\left(\mathbb{R}_{\Gamma_{X}^{\dagger}}^{\dagger} \mathcal{O}_{\mathcal{P}}\left({ }^{\dagger} T\right)_{\mathbb{Q}}[d]\right) \stackrel{\sim}{\longrightarrow} \mathbb{R} \underline{\Gamma}_{X}^{\dagger} \mathcal{O}_{\mathcal{P}}\left({ }^{\dagger} T\right)_{\mathbb{Q}}[d]$ où $d:=-d_{X / P}$ est la codimension de $X$ dans $P$. On en déduit $: \mathbb{D}_{\mathcal{P}, T}\left(\mathbb{R} \underline{\Gamma}_{X}^{\dagger} \mathcal{O}_{\mathcal{P}}\left({ }^{\dagger} T\right)_{\mathbb{Q}}\right)[-2 d] \stackrel{\sim}{\longrightarrow}$ $\mathbb{R} \underline{\Gamma}_{X}^{\dagger} \mathcal{O}_{\mathcal{P}}\left({ }^{\dagger} T\right)_{\mathbb{Q}}$. Cet isomorphisme ne semble pas compatible à Frobenius. En effet, lorsque $X=P$, on retrouve l'isomorphisme canonique : $\mathbb{D}_{\mathcal{P}, T}\left(\mathcal{O}_{\mathcal{P}}\left({ }^{\dagger} T\right)_{\mathbb{Q}}\right) \stackrel{\sim}{\longrightarrow} \mathcal{O}_{\mathcal{P}}\left({ }^{\dagger} T\right)_{\mathbb{Q}}$. Je n'ai pas de contre-exemple mais la compatibilité à Frobenius de ce dernier isomorphisme me paraît inexacte.

Pour tout $\mathcal{E} \in F-D_{\text {coh }}^{\mathrm{b}}\left(\mathcal{D}_{\mathcal{P}}^{\dagger}\left({ }^{\dagger} T\right)_{\mathbb{Q}}\right)$ à support dans $X$, avec les notations 4.3.2, on pose

$$
\mathbb{D}_{X, \mathcal{P}, T}^{*}(\mathcal{E}):=\mathbb{D}_{\mathcal{P}, T}\left(\left(\mathbb{D}_{\mathcal{P}, T}\left(\mathbb{R} \underline{\Gamma}_{X}^{\dagger} \mathcal{O}_{\mathcal{P}}\left({ }^{\dagger} T\right)_{\mathbb{Q}}\right)[-2 d]\right) \stackrel{\mathbb{Q}}{\otimes}_{\mathcal{O}_{\mathcal{P}}\left({ }^{\dagger} T\right)_{\mathbb{Q}}} \mathcal{E}\right)
$$


Or, comme $\mathcal{E}$ est aussi à support dans $X$, on obtient le deuxième isomorphisme :

$$
\mathbb{R} \underline{\Gamma}_{X}^{\dagger} \mathcal{O}_{\mathcal{P}}\left({ }^{\dagger} T\right)_{\mathbb{Q}}{\left.\stackrel{\mathbb{Q}}{\mathbb{O}_{\mathcal{P}}}{ }^{\dagger} T\right)_{\mathbb{Q}}}^{\mathcal{E}} \stackrel{\sim}{\longrightarrow} \mathbb{R} \underline{\Gamma}_{X}^{\dagger}(\mathcal{E}) \stackrel{\sim}{\longrightarrow} \mathcal{E}
$$

Il en découle l'isomorphisme $\mathbb{D}_{X, \mathcal{P}, T}^{*}(\mathcal{E}) \stackrel{\sim}{\longrightarrow} \mathbb{D}_{\mathcal{P}, T}(\mathcal{E})$. Ce dernier ne devrait pas être compatible à Frobenius.

Lemme 4.3.4. - Soient $u: \mathfrak{X} \hookrightarrow \mathcal{P}$ une immersion fermée de $\mathcal{V}$-schémas formels lisses, $T$ un diviseur de $P$ tel que $T_{X}:=u_{0}^{-1}(T)$ soit un diviseur de $X$, $\mathcal{E}^{(\bullet)} \in F-L D_{\mathbb{Q}, \mathrm{qc}}^{\mathrm{b}}\left(\widehat{\mathcal{D}}_{\mathcal{P}}^{(\bullet)}(T)\right), \mathcal{F}^{(\bullet)} \in F-L D_{\mathbb{Q}, \mathrm{qc}}^{\mathrm{b}}\left(\widehat{\mathcal{D}}_{\mathfrak{X}}^{(\bullet)}\left(T_{X}\right)\right)$. Avec les notations de

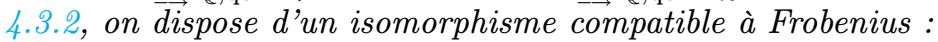

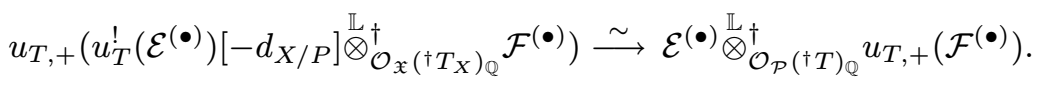

Démonstration. - Par les arguments habituels de complétion puis de passage à la limite sur le niveau, on se ramène au cas des schémas. Par passage de gauche à droite, cela correspond à $[16,1.4 .1]$ et $[16,1.4 .10 .2]$ (on s'était contenté dans $[16,1.4]$ de prouver le cas où $\mathcal{E}=\mathcal{D}_{X}^{(m)}$ et $\mathcal{F}=\omega_{X}$, mais la preuve est identique pour le cas général).

Proposition 4.3.5. - Désignons par $E$ un $F$-isocristal sur $Y$ surconvergent le long de $T_{X}$ et par $E^{\vee}$ son dual. On dispose de l'isomorphisme canonique fonctoriel en $E$ et compatible à Frobenius :

$$
\operatorname{sp}_{X \hookrightarrow \mathcal{P}, T+}\left(E^{\vee}\right) \stackrel{\sim}{\longrightarrow} \mathbb{D}_{X, \mathcal{P}, T}^{*} \circ \operatorname{sp}_{X \hookrightarrow \mathcal{P}, T+}(E) .
$$

Démonstration. - Comme $X$ et $P$ sont lisses, par additivité, il ne coûte rien de supposer $X$ et $P$ intègres. Par application de $\mathbb{D}_{\mathcal{P}, T}$ (on dispose de l'isomorphisme de bidualité qui est compatible à Frobenius [31, II.3.5]) et en échangeant $E$ et $E^{\vee}$, il est équivalent d'obtenir un isomorphisme compatible à Frobenius de la forme :

$\mathbb{D}_{\mathcal{P}, T}\left(\mathbb{R}_{\Gamma_{X}^{\dagger}}^{\dagger} \mathcal{O}_{\mathcal{P}}\left({ }^{\dagger} T\right)_{\mathbb{Q}}\right)[-2 d] \stackrel{\mathbb{L}}{\mathbb{\otimes}_{\mathcal{O}_{\mathcal{P}}(\dagger T)_{\mathbb{Q}}}^{\dagger}} \operatorname{sp}_{X \hookrightarrow \mathcal{P}, T+}\left(E^{\vee}\right) \stackrel{\sim}{\longrightarrow} \mathbb{D}_{\mathcal{P}, T} \operatorname{sp}_{X \hookrightarrow \mathcal{P}, T+}(E)$.

Supposons dans un premier temps que $X \hookrightarrow P$ se relève en une immersion fermée $u: \mathfrak{X} \hookrightarrow \mathcal{P}$ de $\mathcal{V}$-schémas formels lisses. Via [12, 1.2.14.1], le terme de gauche de 4.3.5.1 est isomorphe à

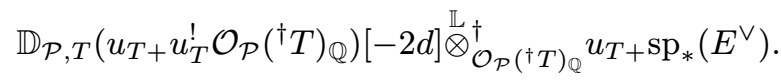

Or, grâce au théorème de dualité relative compatible à Frobenius (voir [16]), (4.3.5.3)

$\mathbb{D}_{\mathcal{P}, T}\left(u_{T+} u_{T}^{!} \mathcal{O}_{\mathcal{P}}\left({ }^{\dagger} T\right)_{\mathbb{Q}}\right)[-2 d] \stackrel{\sim}{\longrightarrow} u_{T+} \mathbb{D}_{\mathfrak{X}, T_{X}} u_{T}^{!}\left(\mathcal{O}_{\mathcal{P}}\left({ }^{\dagger} T\right)_{\mathbb{Q}}\right)[-2 d] \stackrel{\sim}{\longrightarrow} u_{T+} \mathbb{D}_{\mathfrak{X}, T_{X}}\left(\mathcal{O}_{\mathfrak{X}}\left({ }^{\dagger} T_{X}\right)_{\mathbb{Q}}\right)[-d]$. 
Avec 4.3.4, comme $u_{T}^{!} u_{T,+} \stackrel{\sim}{\longrightarrow} i d([6,5.3 .3])$, le terme de gauche de 4.3.5.1 devient isomorphe à

$$
u_{T,+}\left(\mathbb{D}_{\mathfrak{X}, T_{X}}\left(\mathcal{O}_{\mathfrak{X}}\left({ }^{\dagger} T_{X}\right)_{\mathbb{Q}}\right) \otimes_{\mathcal{O}_{\mathfrak{X}}\left({ }^{\dagger} T_{X}\right)_{\mathbb{Q}}} \operatorname{sp}_{*}\left(E^{\vee}\right)\right) .
$$

D'un autre côté, le terme de droite de 4.3.5.1 est isomorphe à

$$
u_{T,+} \mathbb{D}_{\mathfrak{X}, T_{X}} \mathrm{sp}_{*}(E) .
$$

Dans le cas général où $X \hookrightarrow \mathcal{P}$ ne se relève pas, grâce à 4.1 .9 , il en résulte que les deux termes de 4.3.5.1 sont dans l'image essentielle de $\operatorname{sp}_{X \hookrightarrow \mathcal{P}, T+}$.

Soit $\mathcal{P}^{\prime} \subset \mathcal{P} \backslash T$ un ouvert affine de $\mathcal{P}$ tel que $X \cap P^{\prime}$ soit dense dans $X$. Grâce à [30, 4.1.1] et à [25, 4.2.1] (qui est légèrement mieux que [23]), 4.1.1 et 4.1.2, les deux termes de 4.3.5.1 sont isomorphes si et seulement s'ils le sont au dessus de $\mathcal{P}^{\prime}$. On se ramène ainsi au cas où $T$ est vide et $X \hookrightarrow P$ se relève en une immersion fermée $u: \mathfrak{X} \hookrightarrow \mathcal{P}$ de $\mathcal{V}$-schémas formels lisses. Or, d'après [10], on dispose d'un isomorphisme compatible à Frobenius :

$$
\mathbb{D}_{\mathfrak{X}}\left(\mathcal{O}_{\mathfrak{X}, \mathbb{Q}}\right) \otimes_{\mathcal{O}_{\mathcal{P}, \mathbb{Q}}} \operatorname{sp}_{*}\left(E^{\vee}\right) \stackrel{\sim}{\longrightarrow} \mathbb{D}_{\mathfrak{X}} \operatorname{sp}_{*}(E) \text {. }
$$

Via la première partie de la preuve (4.3.5.4 et 4.3.5.5), en appliquant $u_{+}$à 4.3.5.6, on obtient à isomorphisme près 4.3.5.1 (avec $T$ vide).

Remarques 4.3.6. - Si la conjecture [30, 1.2.1.(TC)] était validée, les preuves de 4.1.2 et de 4.3.1 seraient moins techniques et délicates. En effet, en remplaçant [25, 4.2.1] par [30, 1.2.1.(TC)], de manière analogue à ce qui a été fait dans la preuve de 4.3 .5 , on se ramènerait au cas relevable.

Le théorème qui suit fournit une application importante et prometteuse de nos constructions. Pour sa preuve, on pourra consulter [8, 2.2].

ThÉORÈme 4.3.7. - Soit E un F-isocristal unité sur $Y$ surconvergent le long de $T_{X}$. Le faisceau $\operatorname{sp}_{X \hookrightarrow \mathcal{P}, T+}(E)$ est $\mathcal{D}_{\mathcal{P}, \mathbb{Q}^{-}}^{\dagger}$ cohérent.

\section{BIBLIOGRAPHIE}

[1] P. Berthelot - «Cohomologie rigide et théorie des $\mathscr{D}$-modules », in $p$ adic analysis (Trento, 1989), Lecture Notes in Math., vol. 1454, Springer, 1990, p. 80-124.

[2] _ , «Cohérence différentielle des algèbres de fonctions surconvergentes », C. R. Acad. Sci. Paris Sér. I Math. 323 (1996), p. 35-40.

[3] _ _ « Cohomologie rigide et cohomologie rigide à support propre. Première partie », prépublication IRMAR 96-03, 1996.

[4] _ « $\mathscr{D}$-modules arithmétiques. I. Opérateurs différentiels de niveau fini », Ann. Sci. École Norm. Sup. 29 (1996), p. 185-272. 
[5] , « $\mathscr{D}$-modules arithmétiques. II. Descente par Frobenius », Mém. Soc. Math. Fr. (N.S.) 81 (2000).

[6] _ _ «Introduction à la théorie arithmétique des $\mathscr{D}$-modules », Astérisque 279 (2002), p. 1-80.

[7] A. Borel, P.-P. Grivel, B. Kaup, A. Haefliger, B. Malgrange \& F. Ehlers - Algebraic D-modules, Perspectives in Mathematics, vol. 2, Academic Press Inc., 1987.

[8] D. CARo - "Cohérence différentielle des $F$-isocristaux unités », $C . R$. Math. Acad. Sci. Paris 338 (2004), p. 145-150.

[9] _ « tions L», Ann. Inst. Fourier (Grenoble) 54 (2004), p. 1943-1996.

[10] _ gents », Rend. Sem. Mat. Univ. Padova 114 (2005), p. 131-211.

[11] _ « ¿ Dévissages des $F$-complexes de $\mathscr{D}$-modules arithmétiques en $F$ isocristaux surconvergents », Invent. Math. 166 (2006), p. 397-456.

[12] _ « Fonctions $L$ associées aux $\mathscr{D}$-modules arithmétiques. Cas des courbes », Compos. Math. 142 (2006), p. 169-206.

[13] _ « $F$-isocristaux surconvergents et surcohérence différentielle », Invent. Math. 170 (2007), p. 507-539.

[14] _ , "Log-isocristaux surconvergents et holonomie », à paraître dans Compos. Math, 2009.

[15] __ «-modules arithmétiques surholonomes », Ann. Sci. Éc. Norm. Supér. 42 (2009), p. 141-192.

[16] _ _ "Sur la compatibilité à Frobenius de l'isomorphisme de dualité relative », à paraître dans Rend. Sem. Mat. Univ. Padova, 2009.

[17] D. Caro \& N. Tzuzuki - «Overholonomicity of overconvergent Fisocrystals over smooth varieties », prépublication arXiv:0803.2105, 2008.

[18] R. ELKIK - «Solutions d'équations à coefficients dans un anneau hensélien », Ann. Sci. École Norm. Sup. 6 (1973), p. 553-603.

[19] A. Grothendieck - «Éléments de géométrie algébrique. I. Le langage des schémas », Publ. Math. I.H.É.S. 4 (1960).

[20] R. Hartshorne - Residues and duality, Lecture Notes in Math., vol. 20, Springer, 1966.

[21] C. Huyghe - « $\mathscr{D} \mathrm{sp} \dagger(\infty)$-affinité des schémas projectifs », Ann. Inst. Fourier (Grenoble) 48 (1998), p. 913-956.

[22] A. J. DE Jong - «Smoothness, semi-stability and alterations », Publ. Math. I.H.É.S. 83 (1996), p. 51-93. 
[23] K. S. KedLAYA - «Full faithfulness for overconvergent $F$-isocrystals », in Geometric aspects of Dwork theory. Vol. I, II, Walter de Gruyter GmbH \& Co. KG, Berlin, 2004, p. 819-835.

[24] _ « Semistable reduction for overconvergent $F$-isocrystals. I. Unipotence and logarithmic extensions », Compos. Math. 143 (2007), p. 11641212 .

[25] — « Semistable reduction for overconvergent $F$-isocrystals. II. A valuation-theoretic approach », Compos. Math. 144 (2008), p. 657-672.

[26] _ « Semistable reduction for overconvergent $F$-isocrystals. III. Local semistable reduction at monomial valuations », Compos. Math. 145 (2009), p. $143-172$.

[27] _ « Semistable reduction for overconvergent $F$-isocrystals, IV : Local semistable reduction at nonmonomial valuations », prépublication arXiv:0712.3400.

[28] B. Le Stum - Rigid cohomology, Cambridge Tracts in Mathematics, vol. 172, Cambridge Univ. Press, 2007.

[29] C. Noot-Huyghe - «Construction et étude de la transformée de Fourier pour les $\mathcal{D}$-modules arithmétiques », Thèse, université de Rennes I, 1995.

[30] N. TsuzuKI - «Morphisms of $F$-isocrystals and the finite monodromy theorem for unit-root F-isocrystals », Duke Math. J. 111 (2002), p. 385418.

[31] A. Virrion - «Dualité locale et holonomie pour les $\mathscr{D}$-modules arithmétiques », Bull. Soc. Math. France 128 (2000), p. 1-68.

[32] _ _ «race et dualité relative pour les $\mathscr{D}$-modules arithmétiques », in Geometric aspects of Dwork theory. Vol. I, II, Walter de Gruyter GmbH \& Co. KG, Berlin, 2004, p. 1039-1112. 